Dimas Betioli Ribeiro

\title{
ESTUDO E APLICAÇ̃̃O DE UM ELEMENTO DE CONTORNO INFINITO NA ANÁLISE DA INTERAÇÃO SOLO-ESTRUTURA VIA COMBINAÇÃO MEC/MEF
}

Tese apresentada à Escola de Engenharia de São Carlos da Universidade de São Paulo, como parte dos requisitos para a obtenção do Título de Doutor em Engenharia de Estruturas.

Orientador : Professor Doutor João Batista de Paiva 



\section{Agradecimentos}

Em primeiro lugar, agradeço à minha amada esposa Ana Carolina Lorena. Sua ajuda na correção do texto, incentivo desde o princípio e apoio nos momentos mais difíceis foram, sem dúvida, fundamentais para a conclusão deste trabalho.

Agradeço também ao meu orientador João Batista de Paiva, que me deu esta oportunidade e participou ativamente de todo o desenvolvimento desta Tese. Sua ajuda foi muito importante também na publicação de meus trabalhos, que serão valiosos nas etapas seguintes da minha vida. Ao Professor Wilson Sérgio Venturini pela oportunidade de participar do Projeto Alfa nos anos de 2005 e 2006, quando obtive conhecimento importante para o enriquecimento deste trabalho.

Agradeço a todos os alunos e professores envolvidos no Projeto Alfa, cuja participação me ensinou não só sobre elementos de contorno mas também sobre amizade e companheirismo. Agradeço em especial aos colegas Eduardo Toledo de Lima Junior e Manoel Dênis Costa Ferreira.

Agradeço à minha família pelo apoio, em especial aos meus pais Martônio Ribeiro e Orilda Betioli Ribeiro.

Por fim, agradeço a todos os funcionários do SET que contribuíram direta ou indiretamente neste projeto de Doutoramento, por toda a força de vontade e atenção. 


\section{Resumo}

RIBEIRO, D. B. (2009). Estudo e aplicação de um elemento de contorno infinito na análise da interação solo-estrutura via combinação MEC/MEF. 214p. Tese de Doutoramento - Escola de Engenharia de São Carlos, Universidade de São Paulo, São Carlos, 2009.

Neste trabalho, é desenvolvido um programa de computador para a análise estática e tridimensional de problemas de interação solo-estrutura. O programa permite considerar várias camadas de solo, cada qual com características físicas diferentes. Sobre este solo, o qual pode conter estacas, podem ser apoiados diversos tipos de estruturas, tais como placas e até um edifício. Todos os materiais considerados são homogêneos, isotrópicos, elásticos e lineares. O solo tridimensional é modelado com o método dos elementos de contorno (MEC), empregando as soluções fundamentais de Kelvin e uma técnica alternativa na consideração do maciço não-homogêneo. Esta técnica, que é uma contribuição original deste trabalho, é baseada no relacionamento das soluções fundamentais de deslocamento dos diferentes domínios, permitindo que sejam analisados como um único sólido sem a necessidade de equações de equilíbrio e compatibilidade. Isso reduz o sistema de equações final e melhora a precisão dos resultados, conforme comprovado nos exemplos apresentados. Para reduzir o custo computacional sem prejudicar a precisão dos resultados, é utilizada uma malha de elementos de contorno infinitos (ECI) nas bordas da malha de ECs para modelar o comportamento das variáveis de campo em longas distâncias. A formulação do ECI mapeado utilizado é outra contribuição original deste trabalho, sendo baseado em um EC triangular. É demonstrado por meio de exemplos que tal formulação é eficiente para a redução de malha, contribuindo de forma significativa na redução do custo computacional. Todas as estruturas que interagem com o solo, incluindo as de fundação, são simuladas empregando o método dos elementos finitos (MEF). Cada estaca é mode- 
lada como uma linha de carga empregando um único elemento finito com 14 parâmetros nodais, o qual utiliza funções de forma do quarto grau para aproximar os deslocamentos horizontais, do terceiro grau para as forças horizontais e deslocamentos verticais, do segundo grau para as forças cisalhantes verticais e constantes para as reações da base. Este elemento é empregado em outros trabalhos, no entanto os autores utilizam as soluções fundamentais de Mindlin na consideração da presença da estaca no solo. Desta forma, a formulação desenvolvida neste trabalho com as soluções fundamentais de Kelvin pode ser considerada mais uma contribuição original. No edifício, que pode incluir um radier como estrutura de fundação, são utilizados dois tipos de EFs. Os pilares e vigas são simulados com elementos de barra, os quais possuem dois nós e seis graus de liberdade por nó. As lajes e o radier são modelados empregando elementos planos, triangulares e com três nós. Nestes EFs triangulares são superpostos efeitos de membrana e flexão, totalizando também seis graus de liberdade por nó. O acoplamento MEC/MEF é feito transformando as cargas de superfície do MEC em carregamentos nodais reativos no MEF. Além de exemplos específicos nos Capítulos teóricos, um Capítulo inteiro é dedicado a demonstrar a abrangência e precisão da formulação desenvolvida, comparando-a com resultados de outros autores.

Palavras-chave: interação solo/estrutura; elementos de contorno infinitos; solo nãohomogêneo; acoplamento MEC/MEF; edifício. 


\section{Abstract}

RIBEIRO, D. B. (2009). Study and application of an infinite boundary element for soil-structure interaction analysis via FEM/BEM coupling. 214p. PhD Thesis - São Carlos School of Engineering, University of São Paulo, São Carlos, 2009.

In this work, a computer code is developed for the static analysis of three-dimensional soil-structure interaction problems. The program allows considering a layered soil, which may contain piles. This soil may support several structures, such as shells or even an entire building. All materials are considered homogeneous, isotropic, elastic and linear. The three-dimensional soil is modeled with the boundary element method (BEM), employing Kelvin fundamental solutions and an alternative multi-region technique. This technique, which is an original contribution of this work, is based on relating the displacement fundamental solution of the different domains, allowing evaluating them as an unique solid and not requiring compatibility or equilibrium equations. In such a way, the final system of equations is reduced and more accurate results are obtained, as demonstrated in the presented examples. In order to reduce the computational cost maintaining the accuracy, an infinite boundary element (IBE) mesh is employed at the BE mesh limits to model the far field behavior. The mapped IBE utilized, based on a triangular EC, is another original contribution of this work. In the presented examples it is demonstrated that this IBE formulation is efficient for mesh reduction, implying on a significant computational cost reduction. All structures that interact with the soil, including the foundations, are simulated with de finite element method (FEM). The piles are modeled using a one-dimensional 14 parameter finite element, with forth degree shape functions for horizontal displacement approximation, third degree shape functions for horizontal forces and vertical displacement, second degree shape functions for vertical share force, and constant for the base reaction. This element is employed in other works, however the authors utilize Mindlin 
fundamental solutions for the pile presence consideration in the soil. In such a way, the formulation developed in this work with Kelvin fundamental solutions may be considered one more original contribution. The building, which may include a radier as a foundation structure, is modeled using two types os FEs. Piles and beams are simulated using bar FEs with two nodes and six degrees of freedom per node. The radier and pavements are modeled employing plane triangular three-node FEs. In these FEs plate and membrane effects are superposed, totalizing six degrees of freedom per node. FEM/BEM coupling is made by transforming the BEM tractions in nodal reactions in the FEM. Even though specific examples are presented in the theoretical Chapters, a role Chapter is dedicated for demonstrating the formulation accuracy and coverage. In most examples, the results are compared with the ones obtained by other authors.

Key-words: soil-structure interaction; infinite boundary elements; layered soil; FEM/ BEM coupling; building. 


\section{Sumário}

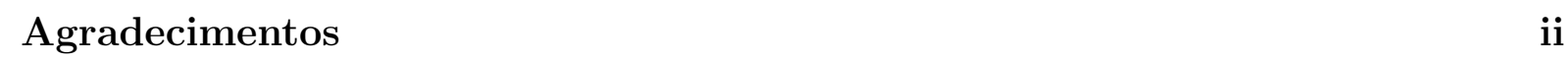

Resumo v v

Abstract vii

$\begin{array}{lll}1 & \text { Introdução } & 1\end{array}$

1.1 Comentários sobre a revisão bibliográfica . . . . . . . . . . . . . . . . . . . 2

1.2 Organização do texto . . . . . . . . . . . . . . . . . . . . . . 13

2 O método dos elementos de contorno 15

2.1 Introdução $\ldots \ldots \ldots \ldots \ldots \ldots$

2.2 Equacionamento básico do problema elástico . . . . . . . . . . . . . . . 15

$2.2 .1 \quad$ O estado de tensão . . . . . . . . . . . . . . . . . . . . . . . . . 15

$2.2 .2 \quad$ O estado de deformação $\ldots \ldots \ldots$. . . . . . . . . . . . . . 19

2.2 .3 Relações constitutivas . . . . . . . . . . . . . . . . . 20

2.3 Soluções fundamentais . . . . . . . . . . . . . . . . . . . . . . . . 21

2.4 Equações integrais de contorno . . . . . . . . . . . . . . . 25

2.4 .1 Equação integral para pontos do contorno . . . . . . . . . . . . . . 28

2.5 Formulação de elementos de contorno . . . . . . . . . . . . . . . . . . . 31

2.5 .1 Transformação de coordenadas . . . . . . . . . . . . . . . . . . 35

2.5 .2 Tratamento da singularidade . . . . . . . . . . . . . . 37

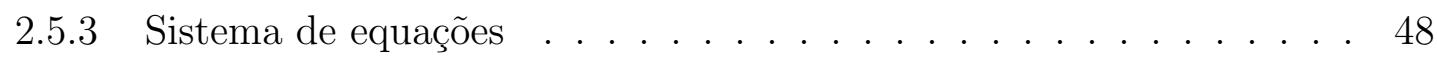

2.5 .4 Movimentos de corpo rígido . . . . . . . . . . . . . . . 50

2.5 .5 Pontos internos . . . . . . . . . . . . . . . . . . . . . . . 51

2.5 .6 Subelementação . . . . . . . . . . . . . . . . . . . . 53 
2.5 .7 Linhas de carga aplicadas ao meio tridimensional $\ldots . . . . . .55$

2.6 Considerações finais $\ldots \ldots \ldots$. . . . . . . . . . . . . . . . . . 65

\begin{tabular}{|lll}
\hline 3 & Sólidos compostos por subregiões & 67
\end{tabular}

3.1 Introdução $\ldots \ldots \ldots \ldots$. . . . . . . . . . . . . . . . . . 67

3.2 Técnica clássica de subregiões $\ldots \ldots$. . . . . . . . . . . . . . . 67

3.3 Técnica alternativa de subregiões $\ldots \ldots \ldots$. . . . . . . . . . . . . . . 69

3.3.1 Sistema de equações . . . . . . . . . . . . . . . . . . . 78

3.3 .2 Linhas de carga combinadas à técnica alternativa $\quad$. . . . . . . . . 79

3.4 Exemplos $\ldots \ldots \ldots \ldots \ldots$

$3.4 .1 \quad$ Sólido composto por dois materiais . . . . . . . . . . . . . . 83

3.4 .2 Sólido composto por quatro materiais . . . . . . . . . . . . . . 86

3.5 Considerações finais $\ldots \ldots \ldots$. . . . . . . . . . . . . . . . . . . . 88

\begin{tabular}{|lll}
\hline & Estudos sobre elementos infinitos & 91
\end{tabular}

4.1 Introdução . . . . . . . . . . . . . . . . . . . . . . . . . . . . 91

4.2 Conceitos iniciais $\ldots \ldots \ldots \ldots$

4.3 Elementos infinitos com funções de decaimento . . . . . . . . . . . . . . . . . 94

$4.3 .1 \quad$ Funções de decaimento exponenciais . . . . . . . . . . . . . . . . 95

$4.3 .2 \quad$ Funções de decaimento recíprocas . . . . . . . . . . . . . . . . . 96

$4.3 .3 \quad$ Integração numérica - Gauss-Legendre . . . . . . . . . . . . . . . . 97

4.4 O elemento infinito mapeado de Zienkiewicz $\ldots \ldots \ldots$. . . . . . . . . . . . . 99

$4.4 .1 \quad$ Elemento infinito unidimensional linear . . . . . . . . . . . . . . . . 105

4.5 Elemento de contorno infinito bidimensional . . . . . . . . . . . . . . . . . 109

4.5 .1 Definição das funções de mapeamento . . . . . . . . . . . . . . . . . 109

4.5 .2 Verificação das funções de mapeamento . . . . . . . . . . . . . . . . 113

4.5 .3 Determinação da posição do pólo . . . . . . . . . . . . . . . . . . 115

4.5 .4 Cálculo do Jacobiano . . . . . . . . . . . . . . . . . . . . . . . . 117

4.5 .5 Inclusão dos ECIs no equacionamento . . . . . . . . . . . . . . . 119

4.5 .6 Tratamento da singularidade nos ECIs . . . . . . . . . . . . . . . 120

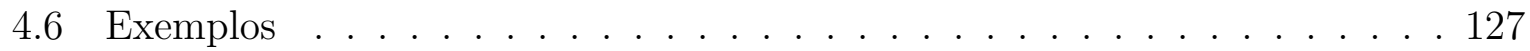

4.6 .1 Semi-espaço infinito homogêneo . . . . . . . . . . . . . . . . 128

4.6 .2 Semi-espaço infinito não homogêneo . . . . . . . . . . . . . . . . . . 130 
4.7 Considerações finais $\ldots \ldots \ldots$. . . . . . . . . . . . . . . . . . . . . . . . . . . .

5 O método dos elementos finitos 135

5.1 Introdução . . . . . . . . . . . . . . . . . . . . . . . . 135

5.2 O Princípio dos Trabalhos Virtuais (PTV) . . . . . . . . . . . . . . . 135

5.3 Elemento finito utilizado nas estacas . . . . . . . . . . . . . . . . . . . . . 140

5.4 Elementos finitos laminares $\ldots \ldots \ldots$. . . . . . . . . . . . . . 149

5.5 Graus de liberdade do elemento finito laminar . . . . . . . . . . . . . . . . 152

5.6 Rotação de eixos . . . . . . . . . . . . . . . . . . . . 154

5.7 Elementos utilizados no edifício . . . . . . . . . . . . . . . 155

5.8 Acoplamento MEC/MEF . . . . . . . . . . . . . . . . . 156

5.9 Considerações finais $\ldots \ldots \ldots$. . . . . . . . . . . . . . . . 164

\begin{tabular}{llr}
\hline 6 & Exemplos & 167
\end{tabular}

6.1 Introdução . . . . . . . . . . . . . . . . . . . . . 167

6.2 Interação de estacas com o solo . . . . . . . . . . . . . . . . 168

$6.2 .1 \quad$ Estaca imersa em um semi-espaço infinito . . . . . . . . . . . . 168

6.2 .2 Nove estacas imersas em um semi-espaço infinito . . . . . . . . . . . 170

6.2 .3 Nove estacas imersas em uma camada finita . . . . . . . . . . . . . 172

6.3 Interação de placas com o solo . . . . . . . . . . . . . . . . 176

6.3 .1 Lâmina apoiada em um semi-espaço infinito . . . . . . . . . . . . 176

6.3 .2 Lâmina apoiada em uma camada finita . . . . . . . . . . . . . . . . 178

6.3 .3 Lâmina apoiada em solo estratificado . . . . . . . . . . . . . . . . . 179

6.4 Interação de placas estaqueadas com o solo . . . . . . . . . . . . . . . . . . 181

6.4 .1 Placa com uma estaca em solo homogêneo . . . . . . . . . . . . . . 181

6.4 .2 Placa com quatro estacas em solo homogêneo . . . . . . . . . . . . 184

6.4 .3 Placa com nove estacas em solo não-homogêneo . . . . . . . . . . . 186

6.5 Edifício apoiado no solo $\ldots \ldots$. . . . . . . . . . . . . . . . . 188

\begin{tabular}{lll}
\hline 7 Conclusões & 195
\end{tabular}

7.1 Observações finais . . . . . . . . . . . . . . . . . . . . . . . . 195

7.2 Propostas para trabalhos futuros f . . . . . . . . . . . . . . 198

\begin{tabular}{ll}
\hline Referências Bibliográficas & 199
\end{tabular} 


\section{Lista de Figuras}

2.1 Sólido tridimensional $\ldots \ldots \ldots$. . . . . . . . . . . . . . . . . . . . . . 16

2.2 Equilíbrio em um elemento infinitesimal 2D $\ldots \ldots \ldots \ldots$

2.3 Equilíbrio em um elemento infinitesimal 3D $\ldots \ldots \ldots \ldots$. . . . . . . . 17

2.4 Equilíbrio de um tetraedro . . . . . . . . . . . . . . . . . . . . 18

2.5 Condições de contorno arbitrárias . . . . . . . . . . . . . . 25

2.6 Ponto $i$ envolvido por uma semi-esfera de raio $\varepsilon$. . . . . . . . . . . . . . . 29

$2.7 \quad$ Elemento de contorno triangular . . . . . . . . . . . . . . . . . . . . . . . 31

2.8 Funções interpoladoras adotadas . . . . . . . . . . . . . . . . . . . 33

2.9 Sistemas de coordenadas global e local . . . . . . . . . . . . . . . . . 33

2.10 Ponto fonte em um dos vértices do elemento . . . . . . . . . . . . . . . . . 37

2.11 Coordenada adimensional $\zeta \ldots \ldots \ldots \ldots \ldots$

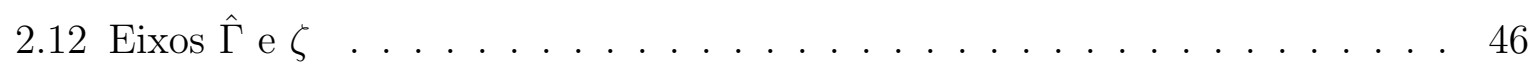

2.13 Subelementação convencional . . . . . . . . . . . . . . . . . . . . 53

2.14 Subelementação progressiva . . . . . . . . . . . . . . . . 54

2.15 Subelemento genérico . . . . . . . . . . . . . . . . . . . . . 54

2.16 Linha de carga genérica . . . . . . . . . . . . . . . 56

2.17 Características das linhas de carga $\ldots \ldots \ldots$. . . . . . . . . . 57

2.18 Projeções do ponto fonte . . . . . . . . . . . . . . . . . . . . . . . 62

2.19 Transformação de coordenadas na base da estaca . . . . . . . . . . . . . . 64

3.1 Duas subregiões em contato . . . . . . . . . . . . . . . . 68

3.2 Três subregiões em contato . . . . . . . . . . . . . . . . . . 70

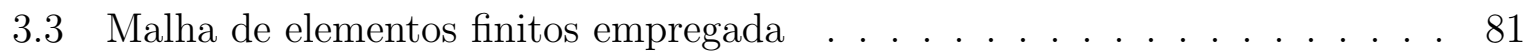

3.4 Primeira malha de elementos de contorno empregada . . . . . . . . . . . 81

3.5 Segunda malha de elementos de contorno empregada . . . . . . . . . . . . 82 
3.6 Terceira malha de elementos de contorno empregada . . . . . . . . . . . . . 82

3.7 Sólido composto por dois materiais . . . . . . . . . . . . . . . . . . 83

3.8 Sólido fletido com dois materiais . . . . . . . . . . . . . . . . . . . . . . . . 85

3.9 Sólido composto por quatro materiais . . . . . . . . . . . . . . . . . 86

3.10 Sólido fletido com quatro materiais $\ldots \ldots$. . . . . . . . . . 87

4.1 Exemplos de elementos infinitos bidimensionais com funções de decaimento 93

4.2 O elemento infinito mapeado de Zienkiewicz . . . . . . . . . . . . . . . . 100

4.3 Funções de mapeamento . . . . . . . . . . . . . . . . . . . . . . . . 104

4.4 Elemento unidimensional linear . . . . . . . . . . . . . . . . . . . . . . 105

4.5 Mapeamento da direção $\xi_{1} \ldots \ldots \ldots$. . . . . . . . . . . . . . . . . . 109

4.6 Mapeamento da direção $\xi_{2}$. . . . . . . . . . . . . . . . . . . . . 110

4.7 Mapeamento das direções $\xi_{1}$ e $\xi_{2} \quad \ldots \ldots \ldots$

4.8 Curva que representa o pólo . . . . . . . . . . . . . . . . . . . . 116

4.9 ECI no sistema de coordenadas globais . . . . . . . . . . . . . . . 120

4.10 ECI no sistema de coordenadas locais oblíquas . . . . . . . . . . . . . . . . 121

4.11 ECI no sistema de coordenadas retangular local . . . . . . . . . . . . . . . 122

4.12 ECI no sistema de coordenadas polares . . . . . . . . . . . . . . . . . 123

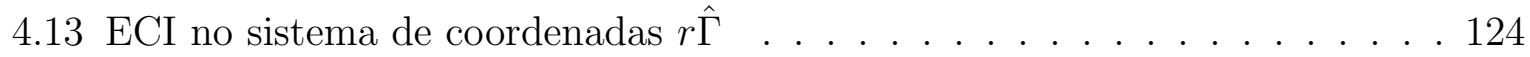

4.14 ECI no sistema de coordenadas $\varsigma$. . . . . . . . . . . . . . . . . . . 125

4.15 Semi-espaço infinito homogêneo com carregamento quadrado . . . . . . . . 128

4.16 Malha de EC/ECI empregada . . . . . . . . . . . . . . . . . . . . . 129

4.17 Semi-espaço infinito não homogêneo com carregamento circular. . . . . . . 131

4.18 Malha da superfície . . . . . . . . . . . . . . . . . . 132

5.1 Sólido qualquer com condições de contorno arbitrárias . . . . . . . . . . . 136

5.2 Características do elemento finito . . . . . . . . . . . . . . 140

5.3 Direção dos deslocamentos e posição dos pontos $P$ e $P^{\prime}$. . . . . . . . . . 150

5.4 Graus de liberdade do elemento finito de membrana FF . . . . . . . . . . . 152

5.5 Graus de liberdade do elemento finito DKT . . . . . . . . . . . . . . . . 153

5.6 Graus de liberdade do elemento finito laminar DKT/FF . . . . . . . . 153

5.7 Sistema de coordenadas global $x_{1} x_{2} x_{3}$ e local $x_{1}^{l} x_{2}^{l} x_{3}^{l} \ldots \ldots$. . . . . 154

5.8 Problema de interação solo-estrutura $\ldots \ldots$. . . . . . . . . . . 156 
5.9 Incompatibilidade entre as cargas de superfície e as cargas nodais . . . . . 158

5.10 Aproximações lineares adotadas para $w$ e $p$. . . . . . . . . . . . . . 160

6.1 Estaca imersa no solo . . . . . . . . . . . . . . . . . . . . . . . . . . . . . . 168

6.2 Malha de EC/ECI empregada . . . . . . . . . . . . . . . . . . . 169

6.3 Deslocamento horizontal devido à carga de $181,6 \mathrm{kN}$. . . . . . . . . . . 169

6.4 Deslocamento horizontal devido ao momento de $95,826 \mathrm{kNm}$. . . . . . . . 170

6.5 Deslocamento vertical devido à carga de $726,4 k N$. . . . . . . . . . . . . . 171

6.6 Nove estacas imersas no solo . . . . . . . . . . . . . . . . . . . . . . . . . . 172

6.7 Malha de EC/ECI empregada . . . . . . . . . . . . . . . . . . . 172

6.8 Deslocamento horizontal da estaca central . . . . . . . . . . . . . . 173

6.9 Nove estacas imersas na camada . . . . . . . . . . . . . . . . . . . . . . . . 174

6.10 Malha de EC/ECI empregada . . . . . . . . . . . . . . . . . . 174

6.11 Deslocamento vertical da estaca central . . . . . . . . . . . . . . . 175

6.12 Lâmina apoiada no solo . . . . . . . . . . . . . . . . . . . . . . . 176

6.13 Malhas de EF/EC/ECI empregadas . . . . . . . . . . . . . . 176

6.14 Lâmina apoiada em meio finito . . . . . . . . . . . . . . . . 178

6.15 Malha de EF/EC/ECI empregada . . . . . . . . . . . . . . . 178

6.16 Lâmina apoiada em meio não-homogêneo . . . . . . . . . . . . . . . . . . 180

6.17 Malha de EF/EC/ECI empregada . . . . . . . . . . . . . . . . 180

6.18 Placa com uma estaca apoiada em meio homogêneo . . . . . . . . . . . . . 181

6.19 Malha de EF/EC/ECI empregada . . . . . . . . . . . . . . . . . 182

6.20 Deslocamento ao longo do eixo $s$. . . . . . . . . . . . . . 183

6.21 Placa quatro estacas apoiada em meio homogêneo . . . . . . . . . . . . . . 184

6.22 Malha de EF/EC/ECI empregada . . . . . . . . . . . . . . . . . . 184

6.23 Deslocamento ao longo do eixo $s$. . . . . . . . . . . . . 186

6.24 Placa estaqueada apoiada em meio não-homogêneo . . . . . . . . . . . . . 187

6.25 Malha de EF/EC/ECI empregada . . . . . . . . . . . . . . . . 188

6.26 Deslocamento vertical das estacas . . . . . . . . . . . . . . . . . . . 189

6.27 Deslocamento vertical para cargas concentradas . . . . . . . . . . . . . . 190

6.28 Interação edifício-radier-estaca-solo . . . . . . . . . . . . . . . . . . . 191

6.29 Cargas verticais aplicadas . . . . . . . . . . . . . . . . . 192

6.30 Malha de EF/EC/ECI empregada . . . . . . . . . . . . . . . . . 192 
6.31 Deslocamento vertical para cargas nas lajes . . . . . . . . . . . . . 193

6.32 Deslocamento vertical no quarto pavimento . . . . . . . . . . . . . . . . . 194

6.33 Cargas horizontais aplicadas . . . . . . . . . . . . . . . . . . . 194

6.34 Deslocamento horizontal no pilar $P 1$. . . . . . . . . . . . . . . . . . . . . 194 


\section{Lista de Tabelas}

$3.1 \quad$ Exemplos do cálculo do coeficiente $c_{l k}^{i} \ldots \ldots \ldots$. . . . . . . . 77

3.2 Sólido com dois materiais e carga axial . . . . . . . . . . . . . . . . . . 84

3.3 Sólido com dois materiais e carga transversal . . . . . . . . . . . . . . . . . 85

3.4 Sólido com quatro materiais e carga axial . . . . . . . . . . . . . . . . 87

3.5 Sólido com quatro materiais e carga transversal . . . . . . . . . . . . . . 88

$4.1 \quad$ Deslocamento vertical $\left(m \times 10^{-2}\right) \ldots \ldots \ldots$. . . . . . . . . . . 129

4.2 Deslocamento vertical para várias malhas $\left(m \times 10^{-2}\right)$. . . . . . . . . . 130

4.3 Deslocamento vertical $\left(m \times 10^{-3}\right) \ldots \ldots$. . . . . . . . . . . 132

$6.1 \quad$ Deslocamento horizontal no topo $\left(m \times 10^{-2}\right) \ldots \ldots$. . . . . . . 173

6.2 Deslocamento vertical $\left(m \times 10^{-8}\right) \ldots \ldots \ldots \ldots \ldots$. . . . . . . . . 177

6.3 Momento fletor nos pontos $A$ e $B$. . . . . . . . . . . . . 177

6.4 Resultados . . . . . . . . . . . . . . . . . . . . . . . . . . . . . 179

6.5 Deslocamentos verticais . . . . . . . . . . . . . . . . . . 181 



\section{Capítulo 1}

\section{Introdução}

Neste trabalho é desenvolvido um programa de computador para a análise estática e tridimensional de problemas de interação solo-estrutura, dando continuidade ao projeto desenvolvido em Ribeiro (2005). O programa permite considerar várias camadas de solo, cada qual com características físicas diferentes. Sobre este solo, o qual pode conter estacas, podem ser apoiados diversos tipos de estruturas, tais como placas e até um edifício. Todos os materiais considerados são homogêneos, isotrópicos, elásticos e lineares.

O solo tridimensional é modelado com o método dos elementos de contorno (MEC), empregando uma técnica alternativa na consideração do maciço não-homogêneo, conforme apresentado em Ribeiro e Paiva (2009). Esta técnica é baseada no relacionamento das soluções fundamentais de deslocamento dos diferentes domínios, permitindo que sejam analisados como um único sólido sem a necessidade de equações de equilíbrio e compatibilidade. Para reduzir o custo computacional sem prejudicar a precisão dos resultados, é utilizada uma malha de elementos de contorno infinitos (ECI) nas bordas da malha de ECs para modelar o comportamento das variáveis de campo em longas distâncias. As funções de mapeamento deste ECI podem ser consultadas em Ribeiro e Paiva (2008).

Todas as estruturas que interagem com o solo, incluindo as de fundação, são simuladas empregando o método dos elementos finitos (MEF). Cada estaca é modelada como uma linha de carga empregando um único elemento finito com 14 parâmetros nodais, de forma semelhante à apresentada em Filho et al. (2005). No edifício, que pode incluir um radier como estrutura de fundação, são utilizados dois tipos de EFs, os quais podem ser também consultados em Ribeiro (2005). Os pilares e vigas são simulados com elementos de barra, enquanto que as lajes e o radier são modelados empregando elementos triangulares planos. 
O acoplamento MEC/MEF é feito transformando as cargas de superfície do MEC em carregamentos nodais reativos no $\mathrm{MEF}$, empregando a mesma estratégia apresentada no trabalho de Filho et al. (2005). Além de exemplos específicos nos Capítulos teóricos, um Capítulo inteiro é dedicado a demonstrar a abrangência e precisão da formulação desenvolvida, comparando-a com resultados de outros autores.

\subsection{Comentários sobre a revisão bibliográfica}

Como a análise da interação do solo com a estrutura de forma integrada é complexa, é comum encontrar na literatura autores que considerem a estrutura isolada, ou seja, sem considerar a influência do solo. No trabalho de Batoz (1980), por exemplo, são estudadas placas fletidas com o emprego do método dos elementos finitos (MEF), mais precisamente com o elemento DKT. Alguns outros trabalhos que também podem ser citados por empregar o MEF na análise de estruturas são Bathe (1982), Oñate (1995), Bezerra (1995) no cálculo de edifícios e Rios (1991) na análise da envoltória de esforços em edifícios altos.

Entre trabalhos mais recentes, Peleteiro (1996) utiliza a teoria do MEF na descrição de um elemento de membrana com graus de liberdade rotacionais nos nós. No trabalho de Mesquita (1998) estruturas são analisadas empregando elementos finitos de casca. O MEF tridimensional é aplicado por Duarte et al. (2000) em problemas de mecânica estrutural. Barros (2002) emprega métodos sem malha e método dos elementos finitos generalizados na análise não-linear de estruturas, simulando a propagação de descontinuidades. Ainda neste contexto, Mendonça (2002) emprega o MEC na análise de estruturas compostas por lâminas planas de espessuras constantes.

Com relação aos trabalhos nos quais considera-se a influência do maciço de solos, em geral, o solo é assumido como sendo homogêneo, isótropo, elástico linear e semiinfinito. Entretanto, na maioria dos casos, torna-se necessária a consideração da nãohomogeneidade do solo e de uma base rígida a uma profundidade prescrita para simular condições mais próximas das encontradas na natureza. Por isto, na literatura, existem técnicas propostas para modelar o solo como um meio finito, estando estas embasadas em diferentes modelos.

Em um deles, o meio contínuo é substituído por um sistema de molas equivalente e 
discreto, também conhecido como modelo de Winkler. Caso haja estaca ela é idealizada como um elemento de viga, com ou sem efeito da deformação axial, apoiado por uma série de molas discretas que representam o solo. As maiores vantagens da aplicação desse modelo são sua simplicidade e relativa facilidade para implementação computacional. Como maior desvantagem, tem-se a dificuldade de escolher-se os módulos de reação das molas para combinações gerais de tamanho de estaca e tipo de solo. Geralmente estes parâmetros são estimados por correlações empíricas e podem levar a soluções incertas e imprecisas. Alguns trabalhos que podem ser citados nesta linha são os de Cheung e Zienkiewicz (1965), Randolph e Wroth (1979), Witt (1984), Lee (1993) e Mylonakis e Gazetas (1998). Estes dois últimos autores apresentam um método simples para cálculo de recalques e tensões de uma estaca ou de grupos de estacas imersas em um meio nãohomogêneo. O solo ao redor da estaca é representado por um modelo generalizado de Winkler, no qual a rigidez em cada profundidade é avaliada empiricamente.

Os trabalhos de Hetenyi (1950), Wang et al. (2001), Kerr (1964) e Kerr (1965) partem do modelo de Winkler em conjunto com modelos contínuos. São introduzidos elementos estruturais para conectar as molas discretas ou então são adotadas simplificações para os modelos contínuos. Estas simplificações são ajustadas por valores reais de deslocamento ou tensão.

Outra técnica que pode ser encontrada na literatura, baseada na teoria da elasticidade, parte do manuseio de equações diferenciais e integrais com o intuito de encontrar soluções para problemas específicos de interação do solo com a estrutura. O solo é geralmente assumido como elástico, linear e homogêneo, simplificando assim o equacionamento. A principal desvantagem desta técnica é que as soluções encontradas se restringem a um pequeno grupo de problemas, não podendo ser aplicadas a casos mais gerais. Relacionado a este contexto pode-se citar o trabalho de Burmister (1945a), no qual é analisado um solo formado por duas camadas. No topo da camada superior é aplicada uma carga externa circular e a espessura da camada inferior é considerada infinita. Partindo do equacionamento da teoria da elasticidade, são obtidas soluções de deslocamento e tensão em pontos internos às camadas. Em Burmister (1945b), esta análise é estendida para três camadas de solo. As soluções de Burmister foram utilizadas por outros autores, podendose citar Poulos (1967), Chan et al. (1974), Davies e Banerjee (1978), Gibson (1967) e Gibson (1974). 
Um modelo conhecido que pode ser aplicado na simulação do solo é o método da camada finita $(\mathrm{MCF})$. Neste método, o MEF é combinado à técnica da transformada de Fourier. Aplicando esta teoria em um problema tridimensional este fica reduzido a apenas duas dimensões, o que reduz o tempo de processamento. Essa ferramenta é eficiente em problemas elásticos, podendo o solo ser formado por camadas de diferentes propriedades físicas e anisotrópico. É possível também considerar estacas imersas no solo, bastando considerar condições de compatibilidade de deslocamentos e equilíbrio de forças na superfície de contato entre o solo e o fuste das estacas. Uma falha que pode ser apontada no MCF é que esta ferramenta pode ser aplicada somente em problemas de domínio elástico. Alguns trabalhos relacionados ao MCF que podem ser citados são Small e Booker (1984), Booker et al. (1989), Lee e Small (1991), Southcott e Small (1996) e Ta e Small (1998). Ainda relacionado a este tema pode ser citado o trabalho de Cheung et al. (1988), no qual é apresentada uma variação do MCF denominada método da camada infinita (MCI).

Por fim, tem-se a linha de pesquisa que se utiliza de métodos numéricos mais avançados, empregando por exemplo o método dos elementos finitos (MEF) ou o método dos elementos de contorno (MEC). O MEF é extremamente versátil e, na maioria dos casos, é a opção mais eficiente e prática para a análise de estruturas. No entanto, as vantagens do MEF são poucas quando se quer analisar situações de domínio infinito, que constitui o caso de problemas de interação do solo com a estrutura. Isto acontece porque o MEF é um método de domínio, sendo necessário dividir o domínio do problema em elementos. Para simular um sólido semi-infinito se torna necessário aplicar as condições de contorno do problema a grandes distâncias, resultando em um grande número de elementos, nós e, consequentemente, equações a serem resolvidas. Além disto, o armazenamento de informações tais como coordenadas de nós e conectividades entre nós e elementos é onerosa. Estes problemas se tornam acentuados principalmente em análises tridimensionais.

Apesar destas desvantagens, o MEF ainda é popular na literatura na simulação de problemas de domínio infinito. Considerando a simulação de túneis, por exemplo, alguns trabalhos que podem ser citados são os de Bae et al. (2005), Kasper e Meschke (2004), Karakus et al. (2007), Zhu et al. (2003), Yin e Yang (2000) e Ulusay et al. (1993). Entre os autores que modelam a interação de estruturas de fundação com o solo, pode-se citar Ottaviani (1975) e Chow e Teh (1991). Neste último, o MEF é aplicado no problema de 
uma placa rígida com estacas apoiada em um solo elástico, linear e finito, estando a placa em contato com o solo. O módulo de elasticidade do solo foi adotado variando linearmente com a profundidade.

Em Fraser e Wardle (1976), é apresentada uma técnica derivada do MEF. Nesta ferramenta, utilizada na análise da interação de uma placa flexível com o solo, somente a porção carregada do solo necessita ser dividida em elementos. O método é intitulado aproximação por elemento de superfície, e se baseia em funções ponderadoras de meio semi-infinito. A placa flexível é abordada pelo MEF convencional.

Um método numérico interessante de ser utilizado em problemas de interação entre solo e estrutura é o chamado método dos elementos infinitos (MEI). Com esta ferramenta, que parte de elementos finitos convencionais, podem ser criados elementos que se estendem até o infinito, tornando desnecessário dividir grandes áreas ou volumes em elementos. Isto é possível por meio de funções de forma especiais, assim a integral sobre o domínio infinito tem valor finito. Estas funções, denominadas na literatura por funções de deterioração (decay functions), geralmente são exponenciais ou logarítmicas.

O primeiro trabalho a citar o termo infinite element foi o de Bettess (1977). Neste trabalho foram utilizadas funções de forma análogas aos polinômios de Lagrange, porém incluindo um termo exponencial de deterioração. Foram satisfeitas duas condições na escolha deste termo: i) representar o problema real de forma coerente; e ii) tornar finito o resultado da integral sobre o elemento. Pela combinação de elementos finitos e infinitos, a formulação foi aplicada a diversos problemas de domínio infinito, e os resultados apresentaram boa concordância com soluções analíticas. O domínio próximo é dividido em elementos finitos que são truncados a uma certa distância. A partir deste limite são utilizados os elementos infinitos representando todo o restante do domínio. Dentre os trabalhos sobre a utilização do método dos elementos infinitos podem ser citados Marques e Owen (1984), Kumar (1985), Yang e Yun (1992), Liu e Novak (1991), Chen e Poulos (1993), Koh e Lee (1998), Yerli et al. (1998), Yun et al. (1999), Kumar (2000), Abdel-Fattah et al. (2000), Sadecka (2000), Yerli et al. (2003) e Liu et al. (2003). Entre os autores brasileiros, cita-se Lopes (2003), Lopes et al. (2003), Lopes et al. (2005), Mesquita e Barros (1995), Barros (1996), Barros e Mesquita (1997), Medeiros (1994) e Medeiros e Raizer (1994).

Outra ferramenta numérica que pode ser considerada eficiente para modelar o solo em 
problemas de interação do solo com a estrutura é o MEC. Como somente o contorno do domínio do problema é dividido em elementos, a análise fica reduzida em uma dimensão. Isto diminui o custo computacional envolvido na resolução de equações, além de simplificar o armazenamento de dados. Devido a estas vantagens vários autores utilizam o MEC na análise da interação do solo com a estrutura, conforme pode ser observado nos trabalhos citados a seguir.

No trabalho de Mindlin (1936), foram obtidas soluções fundamentais em deslocamento e força para uma força concentrada unitária aplicada no interior de um meio semi-infinito homogêneo, elástico, linear e isotrópico. Como estas soluções podem ser empregadas no equacionamento do MEC, vários pesquisadores as utilizam na análise de problemas de semi-espaço infinito.

No trabalho de Poulos (1967) é empregado o modelo de Steinbrenner, utilizando as soluções de Mindlin para calcular valores de deslocamento no interior de um solo apoiado em uma superfície de deslocamento nulo. Neste modelo, escolhe-se a profundidade na qual se encontra a superfície de deslocamento nulo e calcula-se, por Mindlin, o deslocamento de um ponto pertencente a esta superfície. Em seguida determina-se, também por Mindlin, o deslocamento em um ponto qualquer da camada de solo. O deslocamento neste ponto é obtido então pela diferença entre o valor calculado no ponto e o valor calculado na superfície de deslocamento nulo.

O modelo de Steinbrenner foi aplicado novamente em Poulos e Davies (1968), considerando agora uma estaca incompressível imersa no solo. Submetida a uma carga axial, esta estaca é dividida em elementos cilíndricos, cada qual submetido a uma tensão de cisalhamento uniforme. A ponta da estaca é uma base alargada, na qual se considera unicamente a tensão axial.

Esta mesma formulação foi empregada em Poulos (1968), considerando então grupos de estacas. O ponto de partida é a interação de duas estacas, a partir da qual é obtido um coeficiente de influência $\alpha$. Para grupos com mais de duas estacas é feita uma superposição de efeitos, tomando as estacas duas a duas. São analisados grupos simétricos e gerais, sendo as estacas idênticas e estando submetidas ao mesmo carregamento.

Esta abordagem foi utilizada também em Mattes e Poulos (1969), considerando as estacas compressíveis na direção vertical. São calculados deslocamentos verticais utilizando a técnica das diferenças finitas no cálculo de uma equação diferencial. Estes deslocamentos 
são obtidos após a determinação das tensões de cisalhamento ao longo da estaca.

Foi adicionado, em Poulos (1971a), o recurso de aplicar cargas horizontais e momentos no topo de uma estaca isolada. Este procedimento foi então estendido para grupos de estacas em Poulos (1971b).

Em Butterfield e Banerjee (1971) são analisados grupos de estacas ligadas por uma placa rígida. É aplicada uma força concentrada e vertical na placa, determinando então o deslocamento vertical estabelecido no sistema. Em Banerjee (1976) é feito um estudo semelhante, considerando então estacas inclinadas. É utilizado, neste trabalho, o método indireto das equações integrais. A carga aplicada na placa pode ser uma força vertical, horizontal ou um momento. Outra extensão foi adicionada a esse trabalho em Banerjee (1978), tornando possível simular um solo com módulo de elasticidade linearmente variável com a profundidade.

Para considerar duas ou mais camadas de solo com o MEC pode-se empregar diversas técnicas. Uma considerada clássica é descrita em detalhes em Brebbia e Dominguez (1992) e é baseada na imposição de condições de equilíbrio e compatibilidade em todos os pontos pertencentes às superficies de contato. A partir destas relações, as matrizes obtidas para cada domínio empregando o MEC são agrupadas e todos os valores de contorno incógnitos podem ser determinados pela resolução do sistema de equações resultante. Uma desvantagem desta formulação é a presença de blocos de zeros no sistema final. Estes blocos podem se tornar numerosos dependendo do número de domínios considerados, aumentando o tamanho do sistema de equações e por consequência também o tempo de processamento. Além disto, as condições impostas nos contatos não representam a continuidade do meio de forma satisfatória, o que pode prejudicar a precisão dos resultados. Dependendo do problema analisado, outras técnicas da literatura podem se tornar mais atrativas do que essa.

Em Beer (2001) é apresentado um método no qual cada domínio é definido como um superelemento. Usando as matrizes obtidas pelo MEC como ponto de partida, esta formulação consiste em organizar o sistema de equações de cada região de forma semelhante ao método dos elementos finitos (MEF) antes de aplicar as condições de equilíbrio e compatibilidade nos contatos. Isto é vantajoso em casos de acoplamento MEC/MEF, pois esta união se torna mais simples. Em Xiaoping e Wei-liang (2005) uma nova técnica de subregiões é apresentada, na qual o sistema de equações de cada domínio pode ser resolvido 
separadamente. Este método se torna atrativo para problemas que necessitam de malhas muito refinadas, pois ele permite que seja empregado processamento paralelo. Esta formulação foi posteriormente aplicada pelos mesmos autores em problemas que envolvem meios fissurados em Xiaoping e Wei-liang (2006).

O método para subregiões apresentado em Venturini (1992) para problemas bidimensionais elásticos e potenciais elimina a necessidade de condições de equilíbrio e compatibilidade ao longo das interfaces. Esta abordagem melhora a continuidade do meio quando comparada à técnica clássica, implicando em uma maior precisão nos resultados. Essa técnica é modificada em Paiva e Aliabadi (2000) para a análise de placas com o MEC e depois empregada para o cálculo de momentos fletores em placas em Paiva e Aliabadi (2004). Por fim, em Ribeiro e Paiva (2009), esta formulação alternativa foi adaptada para problemas elásticos tridimensionais.

Focando novamente o tema da interação do solo com a estrutura, apresenta-se a seguir alguns trabalhos que simulam o solo com o MEC considerando-o como dois ou mais domínios em contato.

No trabalho de Chin e Chow (1990) o MEC é empregado na análise de grupos de estacas, porém a solução fundamental utilizada na formulação é obtida a partir de Chan et al. (1974). Esta solução corresponde a uma força concentrada horizontal ou vertical aplicada no interior de um solo composto por duas camadas.

Em Pan (1997) é analisado um solo formado por diferentes camadas isotrópicas. Para tornar mais eficiente a implementação da interação das camadas, modeladas pelo MEC, são utilizadas as funções de Green. Uma falha desta ferramenta é a impossibilidade de se considerar elementos de fundação, uma vez que o método permite unicamente a estratificação horizontal das camadas.

Em Maier e Novati (1987), foi desenvolvida uma técnica baseada no MEC para a análise de solos estratificados denominada de método da rigidez sucessiva (MRS). Tratando cada estrato como uma subregião e empregando condições de equilíbrio e compatibilidade entre estratos adjacentes, é possível transferir a rigidez da camada inferior para sua adjacente superior. Desta forma, chega-se à camada da superfície com uma matriz na qual estão incorporadas as influências de todas as camadas inferiores. Este procedimento é então aplicado em exemplos bidimensionais. Na conclusão chega-se a uma matriz final que não é mal-condicionada e cuja resolução demanda um custo computacional inferior 
ao requerido pela técnica convencional de subregiões do MEC. Esse método pode ser aplicado na introdução de outras subregiões, como em análise de túneis ou de elementos de fundação.

Podem ser encontrados também, na literatura, trabalhos que envolvem sólidos elásticos tridimensionais modelados pelo MEC. Estes estudos utilizam as soluções fundamentais de Kelvin, descritas em Love (1944). Em Nakaguma (1979) esta teoria foi empregada em conjunto com as soluções fundamentais de Mindlin (1936) e Boussinesq no estudo de um meio semi-infinito. São apresentados exemplos relacionados à interação do solo com a estrutura. A formulação dispensa a divisão da superfície livre em elementos, tornando a ferramenta versátil. Relacionados à solução fundamental de Kelvin, também podem ser citados Banerjee (1976) e Banerjee e Davies (1977). Estes autores apresentam uma ferramenta para a análise de estacas conectadas ou não por uma placa rígida e imersas em um meio heterogêneo.

Com o intuito de aumentar a abrangência de seus trabalhos, alguns autores estudam o acoplamento de diferentes formulações. Neste contexto, alguns trabalhos podem ser citados por utilizar o MEC em conjunto com o MEF. Segundo Brebbia e Dominguez (1992), entre as diversas técnicas para o acoplamento MEC/MEF, três se destacam. A primeira é resolver isoladamente a parcela MEF do problema e então utilizar os resultados obtidos como condições de contorno na parcela MEC. A desvantagem de utilizar esta abordagem é a impossibilidade de se avaliar de forma completa como os subsistemas interagem entre si, pois a parcela MEC não influi na parcela MEF. A segunda opção, proposta em Brebbia e Georgiou (1979), é representar a parcela MEF com uma formulação semelhante à do MEC, sendo possível então representar o problema acoplado com um único sistema de equações aplicando condições de compatibilidade e equilíbrio entre as subregiões. Esta técnica pode ser considerada mais adequada que a primeira, pois todo o conjunto MEC/MEF é resolvido simultaneamente. A terceira opção, utilizada por Singh et al. (1988) e Swoboda et al. (1987), é representar a parcela MEC de forma semelhante à formulação do MEF, aplicando então condições de compatibilidade e equilíbrio. Esta técnica tem as mesmas vantagens que a segunda, sendo menos custosa computacionalmente. Destaca-se ainda os trabalhos de Meek (1988) e Beer e Meek (1981), por empregarem o acoplamento MEC/MEF na análise tridimensional de problemas geomecânicos.

Na representação do solo em problemas de interação solo-estrutura, conforme apre- 
sentado anteriormente, os dois métodos que se mostram mais eficientes são o método dos elementos infinitos (MEI) e o MEC. Uma desvantagem do MEC sobre o MEI é o fato de que as matrizes resultantes do MEC não possuem simetria e são cheias, enquanto as matrizes do MEI guardam as mesmas características das do MEF. Entretanto no MEC a discretização do domínio não é necessária, o que traz uma grande vantagem para seu uso em relação ao MEI. Neste sentido, a busca de uma formulação que possua as características do MEC e também algumas vantagens do MEI é um caminho natural para representar este problema.

Por este motivo, existem na literatura trabalhos nos quais foram desenvolvidos elementos de contorno infinitos. É o chamado método dos elementos de contorno infinitos (MECI). Na grande maioria dos casos, o elemento de contorno infinito é obtido a partir do elemento convencional, mapeando seu domínio finito em um domínio infinito a partir de funções especiais. Alguns trabalhos podem ser citados nesta linha.

O primeiro trabalho a propor um elemento de contorno infinito foi o de Kagawa et al. (1983). Este autor aproveitou as idéias já existentes do MEI para transformar elementos de contorno convencionais em infinitos, aplicando-os em problemas planos. Desde então, surgiram na literatura muitos outros trabalhos tratando do MECI. Entre eles podem ser citados Kumar (1984), Kagawa et al. (1985), Beer e Watson (1989), Zhang et al. (1989), Zhang et al. (1991), Zhang et al. (1992), Liu e Farris (1993), Chuhan et al. (1995), Davies e $\mathrm{Bu}$ (1996), $\mathrm{Bu}(1996)$ e $\mathrm{Bu}$ (1997). Merecem maior destaque alguns trabalhos mais recentes, apresentados a seguir.

A formulação de Zhang et al. (1999) acopla o MEF, o MEI, o MEC e o MECI para a solução de problemas planos de interação solo-estrutura. O solo, abordado como uma camada finita formada por material não linear, é modelado por elementos finitos e infinitos. A base rochosa da camada de solo é simulada por elementos de contorno finitos e infinitos.

O trabalho de Gao e Davies (1998) emprega elementos de contorno infinitos em problemas tridimensionais de semi-espaço infinito. As integrais singulares são calculadas analiticamente empregando um sistema de coordenadas polares, transformando as integrais de superfície em integrais de linha.

Em Liang e Liew (2001) são empregados elementos de contorno infinitos na análise de um semi-espaço infinito tridimensional. São empregadas soluções fundamentais alternativas, amenizando a singularidade das integrais envolvidas no método. 
O trabalho de Moser et al. (2004) estudou problemas tridimensionais com interação de diferentes subregiões, utilizando o MECI. Sua formulação, aplicada a sólidos semiinfinitos e não-homogêneos, foi comparada com benchmarks da literatura e posteriormente empregada na análise de um problema de interação solo-estrutura envolvendo um túnel.

Entre os pesquisadores brasileiros, pode-se citar vários trabalhos relacionados à interação do solo com estrutura. No trabalho de Aoki e Lopes (1975) é feito um estudo das fundações profundas, determinando deslocamentos e tensões cisalhantes de forma semelhante ao trabalho de Poulos (1968). Esta teoria foi também empregada em Reis (2000) no estudo de fundações rasas.

O trabalho de Gusmão (1990) também utiliza a formulação de Aoki e Lopes (1975). São analisados deslocamentos em um edifício bidimensional, apoiado em um solo estratificado que se encontra sobre uma superfície de deslocamento nulo.

Em Moura (1995) é analisada a interação de um edifício tridimensional com o solo. O edifício, modelado pelo MEF, é composto por elementos reticulares que representam os pilares e vigas e por diafragmas rígidos para simular as lajes. O solo, considerado uma camada homogênea apoiada em uma superfície de deslocamento nulo, é modelado segundo o procedimento apresentado em Aoki e Lopes (1975). A conecção dos pilares do eficício com o solo é feita por meio de elementos de sapata.

No trabalho de Holanda (1998) é empregado o procedimento descrito em Poulos (1967) para simular uma camada de solo homogêneo apoiada em uma superfície de deslocamento nulo. A estrutura considerada é um edifício de concreto armado apoiado em fundações diretas.

Em Antunes e Iwamoto (2000), estuda-se a interação de estacas com um solo estratificado. O solo, apoiado em uma camada de deslocamento nulo, é modelado utilizando a teoria descrita em Aoki e Lopes (1975). São aplicados incrementos de carga na estaca, e sua ponta absorve carga somente após a mobilização de todo o fuste.

Em Mesquita e Coda (2000) é estudado um pórtico plano apoiado sobre uma camada de solo finita e visco-elástica. Nesta análise, o solo é modelado pelo MEC em abordagem bidimensional e a conecção deste com o pórtico é feita por meio de sapatas, sendo possível apoiar cada uma em um meio com propriedades diferentes. Em Leite et al. (2001) é utilizada esta mesma teoria, incluindo elementos enrigecedores no solo representando as estacas. 
No trabalho de Mendonça e Paiva (2003) são analisados grupos de estacas, que podem estar conectadas por uma placa flexível. O solo é considerado um semi-espaço infinito elástico, linear e homogêneo, representado por equações integrais utilizando a solução fundamental de Mindlin. Cada estaca é representada por um único elemento finito reticular com três nós, e a força vertical cisalhante ao longo do fuste é aproximada por uma função quadrática. Na extremidade inferior da estaca a tensão é considerada constante ao longo da Seção transversal, e um dos nós se localiza nesta extremidade. A placa é modelada pelo MEF utilizando dois tipos de elementos finitos planos, o DKT e o HSM. Em Mendonça e Paiva (2000) é feita uma análise semelhante, mas modelando a placa flexível também por equações integrais ao invés de elementos finitos. Também emprega uma formulação semelhante o trabalho de Filho et al. (2005), mas considerando placas rígidas e permitindo que sejam aplicadas cargas horizontais.

Em Almeida e Paiva (2004b) é proposta uma formulação para a análise da interação do solo com a estrutura na qual o solo, composto por uma ou mais camadas apoiadas em uma superfície de deslocamento nulo, é modelado pelo MEC em abordagem tridimensional. Além disto aplica-se no solo o método da rigidez sucessiva (MRS), proposto em Maier e Novati (1987), estendendo-o ao caso tridimensional e à inclusão de subregiões, também tridimensionais. Estas subregiões simulam elementos de fundação tais como estacas, sapatas, tubulões, escavações ou túneis, podendo estes ultrapassar ou não as diferentes camadas de solo. A superestrutura, que pode ser até um edifício tridimensional, é simulada pelo MEF. Os pilares e vigas são representados por elementos reticulares e as lajes são consideradas diafragmas rígidos. O edifício pode ser apoiado em uma placa flexível (radier), que é modelada por elementos finitos laminares. Para avaliar a interação da superestrutura modelada pelo MEF com a subestrutura formulada pelo MEC, é feito o acoplamento a partir das matrizes provenientes de ambos os subsistemas. O resultado é um sistema de equações que representa todo o conjunto.

A formulação de Almeida e Paiva (2004b) foi ponto de partida para os trabalhos de Almeida e Paiva (2007), Ribeiro et al. (2005b), Ribeiro et al. (2005a) e Ribeiro (2005), na análise de problemas de interação do solo com a estrutura. Foram estudados problemas complexos em Ribeiro (2005), tais como a interação de um edifício 3D, modelado pelo MEF, com um solo modelado pelo MEC. As vigas e pilares do edifício foram simulados com elementos de barra, enquanto as lajes foram modeladas com elementos de casca. Os 
resultados obtidos demonstraram a coerência da formulação.

Outros trabalhos envolvendo o tema da interação do solo com a estrutura e que podem ser citados são Silva (1992), Quezado (1993), Silva (1994), Moura (1999), Lorentz (1985), Mello (1984), Veiga (2000), Barretto (1995), Mendonça (1997), Rangel (1997), Iwamoto (2000), Jordão (2003), Almeida (2003a), Oshima (2004), Paccola (2004), Frigerio (2004), Aun (2004), Pereira (2004), Soares (2004) e Neto (2005).

\subsection{Organização do texto}

Iniciando a parte teórica do trabalho, no Capítulo 2 é descrita de forma sucinta a formulação do método dos elementos de contorno aplicado a sólidos tridimensionais.

No Capítulo 3 é apresentada a teoria que está sendo empregada na consideração de sólidos não-homogêneos tridimensionais pelo MEC.

Na sequência apresenta-se, no Capítulo 4, toda a teoria estudada na área de elementos infinitos. Esta teoria é empregada, ao final do Capítulo, no desenvolvimento de um ECI, o qual se utiliza também de conceitos apresentados no Capítulo 2.

O Capítulo 5, por sua vez, contém os conceitos do MEF que foram utilizados neste trabalho para modelar a superestrutura. Esta é formada por lajes e barras, e pode estar apoiada em um radier. Ao final do Capítulo, apresenta-se a técnica utilizada no acoplamento MEC/MEF.

O objetivo do Capítulo 6 é apresentar as principais aplicações do programa computacional desenvolvido. Desta forma, diversos exemplos são apresentados abrangendo desde problemas mais simples, como o de uma estaca isolada, até um edifício tridimensional com suas estruturas de fundação.

No Capítulo 7 são apresentadas as conclusões do trabalho, incluindo observações finais e propostas para trabalhos futuros. 


\section{Capítulo 2}

\section{O método dos elementos de contorno}

\subsection{Introdução}

Este Capítulo tem como objetivo apresentar sucintamente a formulação do método dos elementos de contorno (MEC) para a análise estática de sólidos tridimensionais, homogêneos, isotrópicos, elásticos e lineares. Este modelo é utilizado, neste trabalho, para simular camadas de semi-espaços infinitos e não-homogêneos, nos quais podem ser apoiadas estruturas modeladas com o método dos elementos finitos (MEF). O MEC é descrito de forma semelhante à apresentada em Brebbia e Dominguez (1992).

\subsection{Equacionamento básico do problema elástico}

Dado um sólido, seu comportamento pode ser considerado conhecido caso seja possível determinar o estado de tensão, deformação e deslocamento em qualquer ponto pertencente ao seu domínio. Os dois primeiros são representados por tensores de segunda ordem, descritos a seguir, que podem ser relacionados por equações constitutivas. Estas relações são referentes às características do material do qual o sólido é formado.

\subsubsection{O estado de tensão}

O estado de tensão em um ponto $P$ qualquer de um sólido tridimensional, conforme mostrado na figura 2.1. é composto de nove componentes. Estas componentes podem ser 
agrupadas em um tensor, conforme a expressão 2.1:

$$
[\sigma]=\left[\begin{array}{ccc}
\sigma_{11} & \sigma_{12} & \sigma_{13} \\
\sigma_{21} & \sigma_{22} & \sigma_{23} \\
\sigma_{31} & \sigma_{32} & \sigma_{33}
\end{array}\right]
$$

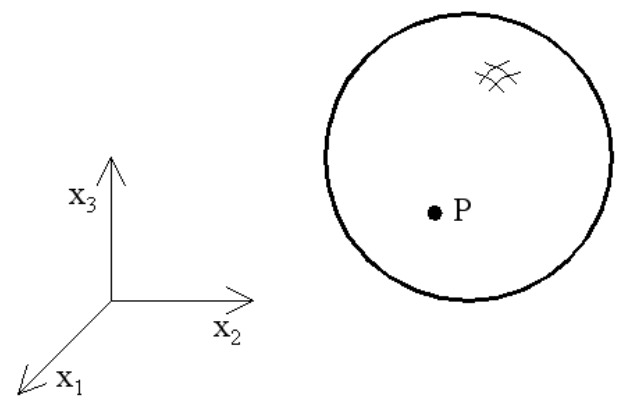

Figura 2.1: Sólido tridimensional

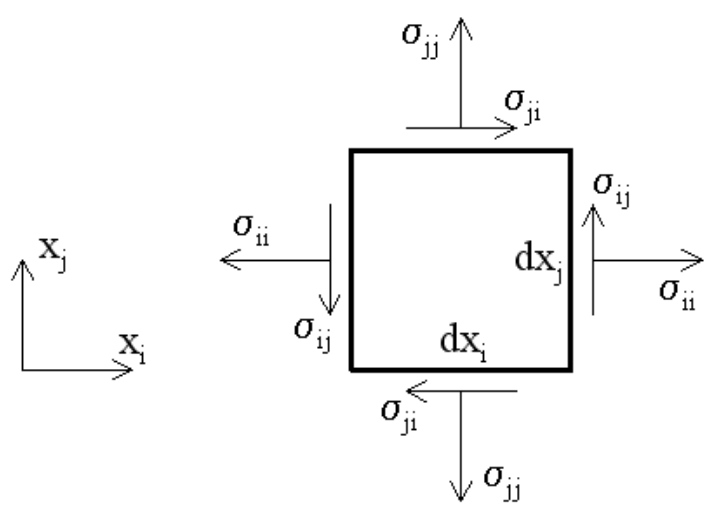

Figura 2.2: Equilíbrio em um elemento infinitesimal 2D

As componentes $\sigma_{i j}$ do tensor 2.1 obedecem condições de equilíbrio, que podem ser em momento ou em força. As equações de equilíbrio em momento podem ser escritas para um elemento infinitesimal bidimensional, conforme ilustrado na figura 2.2. Assim, obtém-se as relações:

$$
\begin{gathered}
\Sigma M=0 \\
\sigma_{i j} d x_{j} d x_{i}-\sigma_{j i} d x_{i} d x_{j}=0 \\
\sigma_{i j}=\sigma_{j i}
\end{gathered}
$$

Em um sólido tridimensional, a relação 2.4 é válida para quaisquer $i, j=1,2,3$. 
Portanto, pode-se escrever as igualdades:

$$
\sigma_{12}=\sigma_{21}, \quad \sigma_{13}=\sigma_{31}, \sigma_{23}=\sigma_{32}
$$

Isto reduz as componentes do estado de tensão de nove para seis. Mais equações podem ser obtidas a partir do equilíbrio de forças nas direções $x_{1}, x_{2}$ e $x_{3}$. A figura 2.3 a ilustra o caso geral de uma parcela infinitesimal de um sólido tridimensional submetida a um estado de tensão e forças de domínio $b_{i}$ quaisquer. Considerando a soma de forças na direção do eixo $x_{1}$, conforme ilustrado na figura $2.3 \mathrm{~b}$, obtém-se a equação:

$$
\sum F x_{1}=0
$$

$\mathrm{Ou}$

$$
\sigma_{11}-\sigma_{11}-\frac{\partial \sigma_{11}}{\partial x_{1}} d x_{1}+\sigma_{12}-\sigma_{12}-\frac{\partial \sigma_{12}}{\partial x_{2}} d x_{1}+\sigma_{13}-\sigma_{13}-\frac{\partial \sigma_{13}}{\partial x_{3}} d x_{1}+b_{1} d x_{1}=0
$$
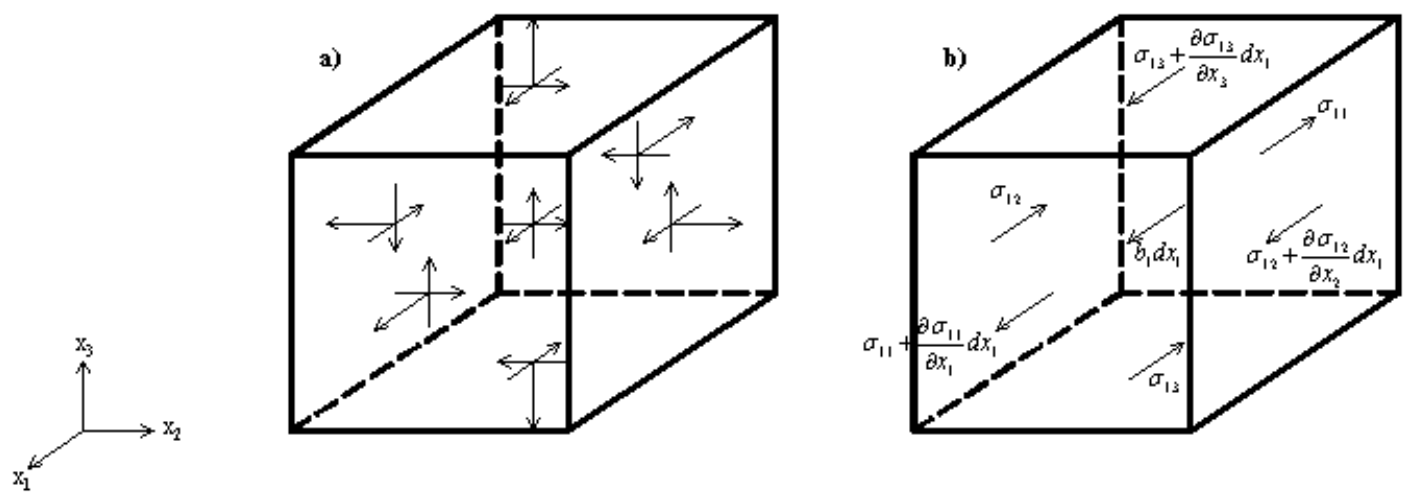

Figura 2.3: Equilíbrio em um elemento infinitesimal 3D

Desenvolvendo-se a equação 2.7 .

$$
\frac{\partial \sigma_{11}}{\partial x_{1}} d x_{1}+\frac{\partial \sigma_{12}}{\partial x_{2}} d x_{1}+\frac{\partial \sigma_{13}}{\partial x_{3}} d x_{1}+b_{1} d x_{1}=0
$$

E, por fim, obtém-se a equação de equilíbrio:

$$
\frac{\partial \sigma_{11}}{\partial x_{1}}+\frac{\partial \sigma_{12}}{\partial x_{2}}+\frac{\partial \sigma_{13}}{\partial x_{3}}+b_{1}=0
$$


O somatório de forças feito para a direção do eixo $x_{1}$ é válido também para as direções $x_{2}$ e $x_{3}$. Portanto:

$$
\begin{aligned}
& \frac{\partial \sigma_{21}}{\partial x_{1}}+\frac{\partial \sigma_{22}}{\partial x_{2}}+\frac{\partial \sigma_{23}}{\partial x_{3}}+b_{2}=0 \\
& \frac{\partial \sigma_{31}}{\partial x_{1}}+\frac{\partial \sigma_{32}}{\partial x_{2}}+\frac{\partial \sigma_{33}}{\partial x_{3}}+b_{3}=0
\end{aligned}
$$

Utilizando notação indicial, as equações 2.9, 2.10 e 2.11 se reduzem à seguinte igualdade:

$$
\sigma_{i j, j}+b_{i}=0
$$

Na sequência, é estudado o equilíbrio de um tetraedro definido pelos planos $x_{1}=0$, $x_{2}=0, x_{3}=0$ e um outro plano qualquer. Para que o tetraedro fique equilibrado em conjunto com o restante do sólido, aparecem componentes de força nas três direções cartesianas. Esta situação pode ser visualizada na figura 2.4.

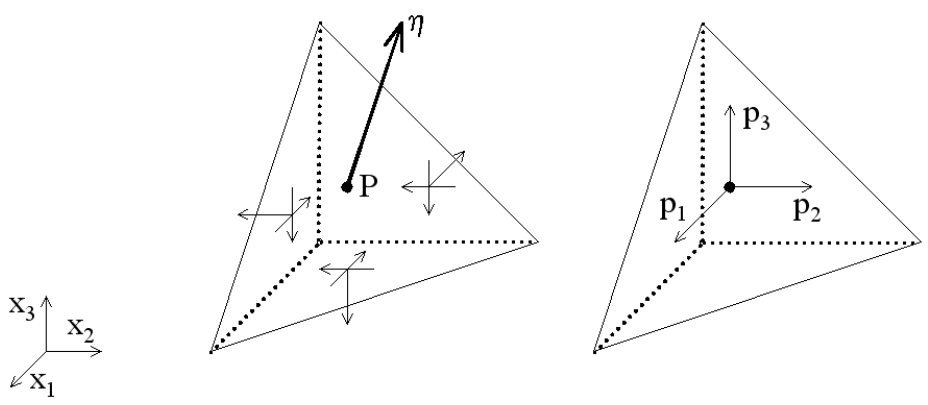

Figura 2.4: Equilíbrio de um tetraedro

Na figura 2.4, $p_{1}, p_{2}$ e $p_{3}$ são as componentes, nas direções dos eixos, da força por unidade de área resultante que equilibra o plano. O versor $\eta$ indica a orientação do plano e também pode ser decomposto nas direções cartesianas, obtendo $\eta_{1}, \eta_{2}$ e $\eta_{3}$. Estas componentes são denominadas co-senos diretores do plano. A partir da figura 2.4, escrevese as expressões:

$$
\begin{aligned}
& p_{1}=\sigma_{11} \eta_{1}+\sigma_{12} \eta_{2}+\sigma_{13} \eta_{3} \\
& p_{2}=\sigma_{21} \eta_{1}+\sigma_{22} \eta_{2}+\sigma_{23} \eta_{3} \\
& p_{3}=\sigma_{31} \eta_{1}+\sigma_{32} \eta_{2}+\sigma_{33} \eta_{3}
\end{aligned}
$$

As relações 2.13, 2.14 e 2.15 podem ser reduzidas a uma única expressão, utilizando notação indicial. Esta expressão é:

$$
p_{i}=\sigma_{i j} \eta_{j}
$$


Os co-senos diretores aparecem multiplicando as componentes do tensor de tensões porque estas atuam em parcelas dos planos $x_{1}=0, x_{2}=0$ e $x_{3}=0$, correspondentes às faces do tetraedro em estudo.

As equações 2.13, 2.14 e 2.15 são válidas para qualquer plano interno do sólido tridimensional em questão, no entanto geralmente são aplicadas ao seu contorno. Este contorno é uma superfície $\Gamma$, na qual deve-se prescrever valores de contorno que podem ser em força ou em deslocamento. No caso tridimensional são seis valores de contorno para cada ponto do contorno $\Gamma$, um deslocamento e uma força para cada direção cartesiana. Dado um ponto e uma direção, deve-se prescrever a força ou o deslocamento, resultando em um valor conhecido e seu conjugado incógnito.

\subsubsection{O estado de deformação}

O campo de deformações pode ser relacionado ao campo de deslocamentos ao qual está sujeito o sólido. Estas relações são mostradas a seguir:

$$
\begin{gathered}
\varepsilon_{11}=\frac{\partial u_{1}}{\partial x_{1}} \\
\varepsilon_{22}=\frac{\partial u_{2}}{\partial x_{2}} \\
\varepsilon_{33}=\frac{\partial u_{3}}{\partial x_{3}} \\
\varepsilon_{12}=\varepsilon_{21}=\frac{1}{2}\left(\frac{\partial u_{1}}{\partial x_{2}}+\frac{\partial u_{2}}{\partial x_{1}}\right) \\
\varepsilon_{13}=\varepsilon_{31}=\frac{1}{2}\left(\frac{\partial u_{1}}{\partial x_{3}}+\frac{\partial u_{3}}{\partial x_{1}}\right) \\
\varepsilon_{23}=\varepsilon_{32}=\frac{1}{2}\left(\frac{\partial u_{2}}{\partial x_{3}}+\frac{\partial u_{3}}{\partial x_{2}}\right)
\end{gathered}
$$

Caso se queira utilizar notação indicial, pode-se reduzir estas seis relações a uma só, que é:

$$
\varepsilon_{i j}=\frac{u_{i, j}+u_{j, i}}{2}
$$

Assim como as tensões, as deformações podem ser agrupadas em um tensor, conforme 
mostrado a seguir:

$$
[\varepsilon]=\left[\begin{array}{lll}
\varepsilon_{11} & \varepsilon_{12} & \varepsilon_{13} \\
\varepsilon_{21} & \varepsilon_{22} & \varepsilon_{23} \\
\varepsilon_{31} & \varepsilon_{32} & \varepsilon_{33}
\end{array}\right]
$$

\subsubsection{Relações constitutivas}

O estado de tensões em um ponto qualquer pode ser relacionado ao estado de deformações no mesmo ponto por meio de relações constitutivas referentes ao material do sólido. Para problemas elásticos e lineares, podem ser definidas as constantes de Lamé $\lambda$ e $\mu$. Com estas constantes, pode-se encontrar o estado de tensões em um ponto a partir do estado de deformações com a expressão:

$$
\sigma_{i j}=\lambda \delta_{i j} \varepsilon_{k k}+2 \mu \varepsilon_{i j}
$$

Na expressão 2.25, o termo $\delta_{i j}$ corresponde à função Delta de Kronecker, sendo que $\delta_{i j}=1$ quando $i=j$ e $\delta_{i j}=0$ quando $i \neq j$. Caso se queira encontrar o estado de deformações a partir do estado de tensões, utiliza-se a expressão:

$$
\varepsilon_{i j}=\frac{-\lambda \delta_{i j}}{2 \mu(3 \lambda+2 \mu)} \sigma_{k k}+\frac{1}{2 \mu} \sigma_{i j}
$$

As constantes de Lamé estão relacionadas ao módulo de elasticidade longitudinal do material e ao coeficiente de Poisson pelas expressões:

$$
\begin{gathered}
\mu=\frac{E}{2(1+\nu)} \\
\lambda=\frac{\nu E}{(1+\nu)(1-2 \nu)}
\end{gathered}
$$

Nas expressões 2.27 e 2.28, $E$ é o módulo de elasticidade longitudinal do material e $\nu$ é o coeficiente de Poisson. As expressões 2.25 e 2.26 podem então ser escritas em função de $E$ e $\nu$, se tornando:

$$
\begin{gathered}
\sigma_{i j}=\frac{E}{(1+\nu)}\left[\frac{\nu}{(1-2 \nu)} \delta_{i j} \varepsilon_{k k}+\varepsilon_{i j}\right] \\
\varepsilon_{i j}=\frac{-\nu}{E} \sigma_{k k} \delta_{i j}+\frac{1+\nu}{E} \sigma_{i j}
\end{gathered}
$$


Na análise feita nesta Seção relativa ao problema elástico, foram definidas três componentes de deslocamento, seis componentes de tensão e seis componentes de deformação, totalizando quinze variáveis a serem determinadas. Para auxiliar na obtenção destas variáveis, foram escritas três equações de equilíbrio, seis equações de compatibilidade entre deslocamentos e deformações e a equação 2.25. Esta última está em notação indicial e representa seis equações constitutivas, somando com as demais quinze equações. Desta forma, com o número de equações igual ao número de incógnitas, torna-se possível determinar todas as componentes de deslocamento, deformação e tensão.

\subsection{Soluções fundamentais}

Nesta Seção são apresentadas as soluções fundamentais para um meio tridimensional, elástico, homogêneo e isotrópico, resultantes de uma força concentrada e unitária aplicada em seu domínio, conforme feito por Kelvin e publicado em Love (1944).

Inicialmente, deve-se reescrever as equações de equilíbrio 2.12 em termos de deslocamentos. Para isto, substitui-se 2.29 em 2.12, obtendo:

$$
\frac{E}{(1+\nu)}\left[\frac{\nu}{(1-2 \nu)} \delta_{l j} \varepsilon_{m m, j}+\varepsilon_{l j, j}\right]+b_{l}=0
$$

Na sequência, substitui-se em 2.31 as relações entre deformação e deslocamento dadas por 2.23. Com isto, chega-se à equação:

$$
\frac{E}{(1+\nu)}\left[\frac{\nu}{(1-2 \nu)} \delta_{l j} u_{m, m j}+\frac{u_{l, j j}}{2}+\frac{u_{j, l j}}{2}\right]+b_{l}=0
$$

Dividindo 2.32 por $\mu$, constante de Lamé definida pela expressão 2.27, obtém-se:

$$
\frac{\nu}{(1-2 \nu)} u_{j, j l}+\frac{u_{l, j j}}{2}+\frac{u_{j, j l}}{2}+\frac{b_{l}}{\mu}=0
$$

Foi utilizada a propriedade $\delta_{l j} u_{m, m j}=u_{m, m l}$, e depois trocou-se o índice mudo $m$ por $j$. Desenvolvendo 2.33, obtém-se a equação:

$$
\frac{1}{(1-2 \nu)} u_{j, j l}+u_{l, j j}+\frac{1}{\mu} b_{l}=0
$$

A expressão 2.34 representa as equações de equilíbrio de Navier, ou equações de equi- 
líbrio em deslocamentos. A solução fundamental de Kelvin é obtida a partir da equação 2.34 quando uma força concentrada unitária é aplicada em um ponto $i$ na direção de um versor $e_{l}$, ou seja:

$$
b_{l}=\Delta^{i} e_{l}
$$

em que o termo $\Delta^{i}$ representa a função Delta de Dirac. Para tornar mais simples a dedução da solução fundamental, os deslocamentos podem ser representados pelo vetor de Galerkin. O deslocamento em uma determinada direção $j$ pode ser obtido a partir do vetor de Galerkin $G$ pela expressão:

$$
u_{j}=G_{j, m m}-\frac{1}{2(1-\nu)} G_{m, j m}
$$

onde $G$ é um vetor a ser determinado. Para prosseguir com o equacionamento, deve-se substituir as expressões 2.35 e 2.36 em 2.34. Assim:

$$
\frac{1}{(1-2 \nu)}\left[G_{j, m m j l}-\frac{1}{2(1-\nu)} G_{m, j m j l}\right]+\left[G_{l, m m j j}-\frac{1}{2(1-\nu)} G_{m, j m j j}\right]+\frac{1}{\mu} \Delta^{i} e_{l}=0
$$

Como o índice $m$ é mudo, ele pode ser trocado por qualquer outro. Na equação 2.37 , troca-se alguns índices $m$ por $j$ e outros por $l$, de forma conveniente. Após a troca de índices, a equação 2.37 se torna:

$$
\frac{1}{(1-2 \nu)}\left[G_{j, j j j l}-\frac{1}{2(1-\nu)} G_{j, j j j l}\right]+\left[G_{l, j j l l}-\frac{1}{2(1-\nu)} G_{j, j j j l}\right]+\frac{1}{\mu} \Delta^{i} e_{l}=0
$$

Desenvolvendo a expressão 2.38, obtém-se a equação:

$$
\nabla^{2}\left(\nabla^{2} G_{l}\right)+\frac{1}{\mu} \Delta^{i} e_{l}=0
$$

Para problemas de estado plano de deformação, a expressão 2.39 pode ser escrita como:

$$
\nabla^{2}\left(F_{l}\right)+\frac{1}{\mu} \Delta^{i} e_{l}=0
$$

em que

$$
F_{l}=\nabla^{2} G_{l}
$$

A equação 2.40 é semelhante à que representa o problema potencial em Brebbia e 
Dominguez (1992). Por isto, ela admite a seguinte solução:

$$
F_{l}=\frac{1}{4 \pi r \mu} e_{l}
$$

em que $r$ é a distância entre o ponto de aplicação da carga unitária e o ponto para o qual $F_{l}$ está sendo calculado. O ponto de aplicação da carga é denominado "ponto fonte" e o ponto no qual é feito o cálculo é denominado "ponto campo". Substituindo-se a expressão 2.42 em 2.41, obtém-se:

$$
\nabla^{2} G_{l}=\frac{1}{4 \pi r \mu} e_{l}
$$

A solução desta equação é dada por:

$$
G_{l}=\frac{1}{8 \pi \mu} r e_{l}
$$

Tomando-se cada carga separadamente, pode-se escrever a igualdade:

$$
G_{l k}=G \delta_{l k}
$$

em que

$$
G=\frac{1}{8 \pi \mu} r
$$

e $\delta_{l k}$ é a função Delta de Kronecker. O índice $k$ se refere à componente do vetor de Galerkin e $l$ é a direção da carga unitária aplicada no ponto $i$. O deslocamento em um ponto qualquer do domínio, considerando cada direção como independente, pode ser escrito como:

$$
u_{k}^{*}=u_{l k}^{*} e_{l}
$$

em que $u_{k}^{*}$ é o deslocamento em qualquer ponto na direção $k$, quando é aplicada uma carga unitária no ponto $i$ e na direção $l$. De acordo com a definição dada por 2.36, pode-se escrever:

$$
u_{l k}^{*}=G_{l k, m m}-\frac{1}{2(1-\nu)} G_{l m, k m}
$$

O passo seguinte é substituir 2.45 e 2.46 em 2.48, obtendo:

$$
u_{l k}^{*}=\frac{1}{16 \pi \mu(1-\nu) r}\left[(3-4 \nu) \delta_{l k}+r_{, l} r_{, k}\right]
$$


A expressão 2.49 correponde à solução fundamental de deslocamento de Kelvin. Os termos do tipo $r_{, i}$, correspondentes à derivada de $r$ em relação a uma coordenada $i$, podem ser calculados, segundo Almeida (2003b), da seguinte forma:

$$
r_{, i}=\frac{r_{i}}{r}
$$

em que $r_{i}$ é a componente da distância $r$ na direção da coordenada $i$, podendo ser obtida a partir das coordenadas dos pontos fonte e campo. Denominando o ponto fonte por $s$ e o ponto campo por $p$ :

$$
r_{i}=\left|x_{i}(s)-x_{i}(p)\right|
$$

O tensor de tensões em qualquer ponto interno pode ser encontrado substituindo a expressão 2.49 em 2.23 e o resultado em 2.29. A expressão resultante, representada em notação indicial, é:

$$
\sigma_{k j}^{*}=S_{l k j}^{*} e_{l}
$$

em que $S_{l k j}^{*}$ é um tensor. Substituindo-se a expressão 2.52 em 2.16, obtém-se o valor da tração em um ponto qualquer do contorno $\Gamma$, que é:

$$
p_{k}^{*}=p_{l k}^{*} e_{l}
$$

em que as componentes de tensão para o caso tridimensional são:

$$
p_{l k}^{*}=\frac{-1}{8 \pi(1-\nu) r^{2}}\left[r_{, \eta}\left[(1-2 \nu) \delta_{l k}+3 r_{, l} r_{, k}\right]+(1-2 \nu)\left(\eta_{l} r_{, k}-\eta_{k} r_{, l}\right)\right]
$$

A expressão 2.54 corresponde à solução fundamental de força de Kelvin. O termo $r_{, \eta}$ é aqui calculado da forma descrita em Almeida (2003b), empregando-se a seguinte expressão:

$$
r_{, \eta}=r_{, i} x_{i, \eta}
$$

É empregada notação indicial na igualdade 2.55 , sendo portanto uma soma em $i$ para as três direções. O termo $r_{, i}$ é calculado pela igualdade 2.50 e $x_{i, \eta}=\eta_{i}$. O termo $\eta_{i}$, assim como os termos $\eta_{l}$ e $\eta_{k}$ da expressão 2.54, são componentes relativas ao versor $\eta$, normal ao contorno $\Gamma$. A direção e sentido de $\eta$ podem ser determinados, portanto, a partir da geometria do contorno $\Gamma$, a qual é conhecida. Esta geometria é aproximada, neste 
trabalho, por um conjunto de domínios planos, denominados elementos de contorno, os quais são descritos na Seção 2.5. O versor $\eta$ deve ser então determinado para cada um destes elementos, e a forma como isto é feito pode ser consultada em Oñate (1995).

\subsection{Equações integrais de contorno}

Considera-se um sólido tridimensional, conforme ilustrado na figura 2.5. Nesta figura, o domínio do sólido é tratado por $\Omega$ e seu cotorno por $\Gamma$. Os pontos $s$ e $P$ são, respectivamente, o ponto fonte e o ponto campo. O ponto fonte é onde a carga unitária está aplicada e o ponto campo é onde se quer calcular valores. O contorno do sólido está dividido em dois trechos. No primeiro, denominado $\Gamma_{1}$, estão definidas as condições de contorno essenciais do problema. Isto significa que o deslocamento está prescrito em $\Gamma_{1}$, ou seja:

$$
u_{i}=\overline{u_{i}}
$$
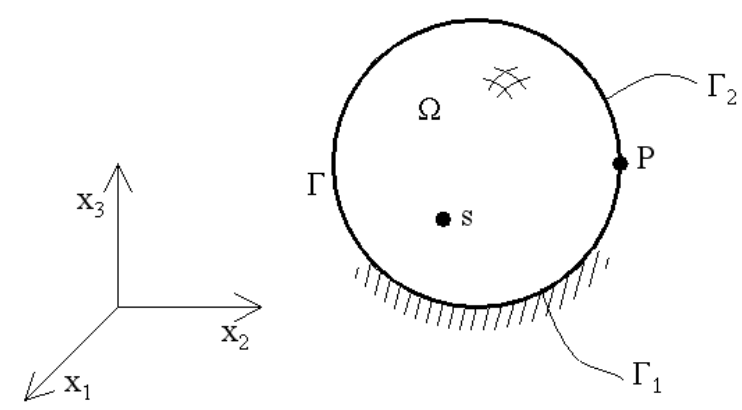

Figura 2.5: Condições de contorno arbitrárias

No segundo trecho, denominado $\Gamma_{2}$, estão definidas as condições de contorno naturais do problema. Isto significa que em $\Gamma_{2}$ foi prescrito força, isto é:

$$
p_{i}=\overline{p_{i}}
$$

A equação integral governante do problema elástico será obtida pelo uso de técnicas de resíduos ponderados. O ponto de partida é a equação diferencial de equilíbrio em tensões deduzida anteriormente, que é:

$$
\sigma_{i j, j}+b_{i}=0
$$


Aplicando resíduos ponderados em 2.58, com uma função ponderadora em deslocamentos $u_{k}^{*}$, chega-se à expressão:

$$
\int_{\Omega}\left(\sigma_{k j, j}+b_{k}\right) u_{k}^{*} d \Omega=0
$$

A equação 2.59 também pode ser escrita como:

$$
\int_{\Omega}\left[\frac{\partial \sigma_{k 1}}{\partial x_{1}} u_{k}^{*}+\frac{\partial \sigma_{k 2}}{\partial x_{2}} u_{k}^{*}+\frac{\partial \sigma_{k 3}}{\partial x_{3}} u_{k}^{*}+b_{k} u_{k}^{*}\right] d \Omega=0
$$

Integrando 2.60 por partes, obtém-se:

$$
-\int_{\Omega}\left[\sigma_{k 1} \frac{\partial u_{k}^{*}}{\partial x_{1}}+\sigma_{k 2} \frac{\partial u_{k}^{*}}{\partial x_{2}}+\sigma_{k 3} \frac{\partial u_{k}^{*}}{\partial x_{3}}+b_{k} u_{k}^{*}\right] d \Omega+\int_{\Gamma} p_{k} u_{k}^{*} d \Gamma=0
$$

Na passagem de 2.60 para 2.61, foi utilizada a definição:

$$
\sigma_{k j} \eta_{j}=p_{k}
$$

Utilizando as equações de compatibilidade entre deslocamentos e deformações definidas pela expressão 2.23, pode-se reescrever 2.61 como:

$$
-\int_{\Omega} \sigma_{k j} \varepsilon_{k j}^{*} d \Omega+\int_{\Omega} b_{k} u_{k}^{*} d \Omega=-\int_{\Gamma} p_{k} u_{k}^{*} d \Gamma
$$

O teorema de Betti garante que:

$$
\int_{\Omega} \sigma_{k j} \varepsilon_{k j}^{*} d \Omega=\int_{\Omega} \sigma_{k j}^{*} \varepsilon_{k j} d \Omega
$$

Aplicando 2.64 em 2.63, obtém-se:

$$
-\int_{\Omega} \sigma_{k j}^{*} \varepsilon_{k j} d \Omega+\int_{\Omega} b_{k} u_{k}^{*} d \Omega=-\int_{\Gamma} p_{k} u_{k}^{*} d \Gamma
$$

Integrando 2.65 por partes chega-se à seguinte equação:

$$
\int_{\Omega} \sigma_{k j, j}^{*} u_{k} d \Omega+\int_{\Omega} b_{k} u_{k}^{*} d \Omega=-\int_{\Gamma} p_{k} u_{k}^{*} d \Gamma+\int_{\Gamma} u_{k} p_{k}^{*} d \Gamma
$$


Imaginando o contorno $\Gamma$ dividido nas parcelas $\Gamma_{1}$ e $\Gamma_{2}$ nas quais são válidas as condições de contorno 2.56 e 2.57, reescreve-se 2.66 como:

$$
\int_{\Omega} \sigma_{k j, j}^{*} u_{k} d \Omega+\int_{\Omega} b_{k} u_{k}^{*} d \Omega=\int_{\Gamma_{1}} \bar{u}_{k} p_{k}^{*} d \Gamma+\int_{\Gamma_{2}} u_{k} p_{k}^{*} d \Gamma-\int_{\Gamma_{1}} p_{k} u_{k}^{*} d \Gamma-\int_{\Gamma_{2}} \bar{p}_{k} u_{k}^{*} d \Gamma
$$

Na sequência, deve-se integrar 2.67 por partes e aplicar o teorema de Betti. Assim, integrando 2.67 por partes, chega-se à equação:

$$
\int_{\Omega} \sigma_{k j}^{*} \varepsilon_{k j} d \Omega+\int_{\Omega} b_{k} u_{k}^{*} d \Omega=\int_{\Gamma_{1}}\left(\bar{u}_{k}-u_{k}\right) p_{k}^{*} d \Gamma-\int_{\Gamma_{1}} p_{k} u_{k}^{*} d \Gamma-\int_{\Gamma_{2}} \bar{p}_{k} u_{k}^{*} d \Gamma
$$

Aplicando o teorema de Betti em 2.68, obtém-se:

$$
\int_{\Omega} \sigma_{k j} \varepsilon_{k j}^{*} d \Omega+\int_{\Omega} b_{k} u_{k}^{*} d \Omega=\int_{\Gamma_{1}}\left(\bar{u}_{k}-u_{k}\right) p_{k}^{*} d \Gamma-\int_{\Gamma_{1}} p_{k} u_{k}^{*} d \Gamma-\int_{\Gamma_{2}} \bar{p}_{k} u_{k}^{*} d \Gamma
$$

Integrando 2.69 por partes, obtém-se a igualdade:

$$
\int_{\Omega} \sigma_{k j, j} u_{k}^{*} d \Omega+\int_{\Omega} b_{k} u_{k}^{*} d \Omega=\int_{\Gamma_{1}}\left(\bar{u}_{k}-u_{k}\right) p_{k}^{*} d \Gamma+\int_{\Gamma_{2}}\left(p_{k}-\bar{p}_{k}\right) u_{k}^{*} d \Gamma
$$

A expressão 2.70 pode ser utilizada na obtenção das equações integrais de contorno. Para isto, deve-se aplicar as soluções fundamentais deduzidas anteriormente nesta expressão. Deste modo, a carga externa a ser considerada é uma força concentrada unitária aplicada em um ponto $i$ do domínio $\Omega$. A partir desta hipótese, a equação diferencial de equilíbrio em tensões 2.58 pode ser escrita como:

$$
\sigma_{k j, j}^{*}+\Delta^{i} e_{l}=0
$$

$\mathrm{Ou}$

$$
\sigma_{k j, j}^{*}=-\Delta^{i} e_{l}
$$

em que $\Delta^{i}$ é a função Delta de Dirac concentrada no ponto $i$ de aplicação da carga. A igualdade 2.72 pode ser utilizada na primeira integral à esquerda de 2.70, que se torna:

$$
\int_{\Omega} \sigma_{k j, j}^{*} u_{k} d \Omega=\int_{\Omega}\left(-\Delta^{i} e_{l}\right) u_{l} d \Omega=-u_{l}^{i} e_{l}
$$


Na igualdade 2.73, $u_{l}^{i}$ é o deslocamento no ponto $i$ onde a carga unitária foi aplicada e na direção $l$. Substituindo 2.73 em 2.70, chega-se à equação:

$$
u_{l}^{i} e_{l}+\int_{\Gamma_{1}} \bar{u}_{k} p_{l k}^{*} d \Gamma e_{l}+\int_{\Gamma_{2}} u_{k} p_{l k}^{*} d \Gamma e_{l}=\int_{\Gamma_{1}} p_{k} u_{l k}^{*} d \Gamma e_{l}+\int_{\Gamma_{2}} \bar{p}_{k} u_{l k}^{*} d \Gamma e_{l}+\int_{\Omega} b_{k} u_{l k}^{*} d \Omega e_{l}
$$

O versor $e_{l}$ aparece em todos os termos, portanto pode ser excluído. Assim:

$$
u_{l}^{i}+\int_{\Gamma_{1}} \bar{u}_{k} p_{l k}^{*} d \Gamma+\int_{\Gamma_{2}} u_{k} p_{l k}^{*} d \Gamma=\int_{\Gamma_{1}} p_{k} u_{l k}^{*} d \Gamma+\int_{\Gamma_{2}} \bar{p}_{k} u_{l k}^{*} d \Gamma+\int_{\Omega} b_{k} u_{l k}^{*} d \Omega
$$

A equação 2.75 pode ser escrita de forma mais compacta caso as condições de contorno sejam, por enquanto, ignoradas. Desta forma:

$$
u_{l}^{i}+\int_{\Gamma} u_{k} p_{l k}^{*} d \Gamma=\int_{\Gamma} p_{k} u_{l k}^{*} d \Gamma+\int_{\Omega} b_{k} u_{l k}^{*} d \Omega
$$

A equação 2.76 é chamada de identidade de Somigliana. Por meio dela, é possível determinar componentes de deslocamento em qualquer ponto do domínio $\Omega$ em função dos valores de contorno $u_{k}$ e $p_{k}$. É necessário que também sejam conhecidas as soluções fundamentais em deslocamento $u_{l k}^{*}$ e força $p_{l k}^{*}$, que foram deduzidas anteriormente, além das cargas externas de domínio $b_{k}$.

\subsubsection{Equação integral para pontos do contorno}

Para determinar as componentes de deslocamento em um ponto $i$ do domínio $\Omega$ a partir da identidade de Somigliana 2.76, devem ser conhecidos os valores de contorno dados por $u_{k}$ e $p_{k}$. Portanto, para calcular valores em pontos internos do sólido devese primeiramente resolver o problema do valor de contorno. Como a equação 2.76 é válida somente no domínio $\Omega$ e não no contorno $\Gamma$, deve-se escrevê-la para um ponto do domínio e fazer este ponto tender ao contorno. Neste procedimento, cada termo deve ser analisado separadamente para tratar de forma especial as integrais que se tornem singulares. Para maior simplicidade, inicialmente é considerado que o contorno $\Gamma$ é suave no ponto $i$ analisado. Com isto, torna-se possível englobar o ponto $i$ ao interior de $\Omega$ por meio de uma semi-esfera, cujo raio $\varepsilon$ será posteriormente levado a zero no limite. Isto está ilustrado na figura 2.6 . 


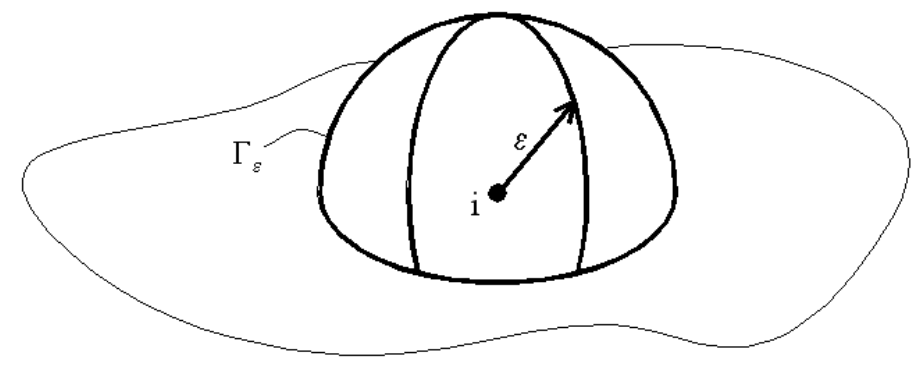

Figura 2.6: Ponto $i$ envolvido por uma semi-esfera de raio $\varepsilon$

Iniciando a análise, toma-se a primeira integral à direita da equação 2.76. Conforme ilustrado na figura 2.6, a semi-esfera contribui com uma parcela $\Gamma_{\varepsilon}$ do contorno $\Gamma$. Assim, esta integral pode ser dividida em duas partes:

$$
\int_{\Gamma} u_{l k}^{*} p_{k} d \Gamma=\lim _{\varepsilon \rightarrow 0}\left\{\int_{\Gamma-\Gamma_{\varepsilon}} u_{l k}^{*} p_{k} d \Gamma\right\}+\lim _{\varepsilon \rightarrow 0}\left\{\int_{\Gamma_{\varepsilon}} u_{l k}^{*} p_{k} d \Gamma\right\}
$$

$\mathrm{O}$ primeiro limite à direita de 2.77 torna $\Gamma_{\varepsilon}$ infinitamente menor que $\Gamma$, o que leva à conclusão de que este limite é igual à própria integral inicial. O segundo limite à direita pode ser escrito como:

$$
\lim _{\varepsilon \rightarrow 0}\left\{\int_{\Gamma_{\varepsilon}} u_{l k}^{*} p_{k} d \Gamma\right\}=p_{k}^{i} \lim _{\varepsilon \rightarrow 0}\left\{\int_{\Gamma_{\varepsilon}} u_{l k}^{*} d \Gamma\right\}
$$

em que $p_{k}^{i}$ é uma força no ponto $i$ na direção $k$. A solução fundamental $u_{l k}^{*}$ tem dimensão $1 / r$ ou $1 / \varepsilon$, conforme demonstrado na expressão 2.49. Como a integral de superfície na expressão 2.77 produz um termo $\varepsilon^{2}$ multiplicando a expressão, restará do desenvolvimento desta equação um $\varepsilon$ multiplicando toda a expressão. Isto é suficiente para concluir que esse termo tende a zero no limite. Portanto, a primeira integral à direita de 2.76 não sofre influência da singularidade no ponto $i$.

A próxima integral a ser analisada é a da esquerda da expressão 2.76 .

$$
\int_{\Gamma} p_{l k}^{*} u_{k} d \Gamma=\lim _{\varepsilon \rightarrow 0}\left\{\int_{\Gamma-\Gamma_{\varepsilon}} p_{l k}^{*} u_{k} d \Gamma\right\}+\lim _{\varepsilon \rightarrow 0}\left\{\int_{\Gamma_{\varepsilon}} p_{l k}^{*} u_{k} d \Gamma\right\}
$$

Analogamente à igualdade 2.77, o primeiro limite à direita de 2.79 é igual à integral à 
esquerda. O segundo limite à direita, por sua vez, pode ser escrito como:

$$
\lim _{\varepsilon \rightarrow 0}\left\{\int_{\Gamma_{\varepsilon}} p_{l k}^{*} u_{k} d \Gamma\right\}=u_{k}^{i} \lim _{\varepsilon \rightarrow 0}\left\{\int_{\Gamma_{\varepsilon}} p_{l k}^{*} d \Gamma\right\}
$$

A solução fundamental $p_{l k}^{*}$, dada pela expressão 2.54, tem dimensão $1 / r^{2}$, enquanto a integral de superfície de 2.80 produz um termo $\varepsilon^{2}$ multiplicando toda a expressão. Isto significa que o limite 2.80 não é igual a zero, e sim um termo livre. Para encontrar este termo é necessário desenvolver analiticamente a integral 2.80. Após os procedimentos matemáticos, chega-se à igualdade:

$$
\lim _{\varepsilon \rightarrow 0}\left\{\int_{\Gamma_{\varepsilon}} p_{l k}^{*} d \Gamma\right\}=-\frac{1}{2} \delta_{l k}
$$

Portanto, a expressão 2.79 se torna:

$$
\int_{\Gamma} p_{l k}^{*} u_{k} d \Gamma=\int_{\Gamma} p_{l k}^{*} u_{k} d \Gamma-\frac{1}{2} \delta_{l k} u_{k}^{i}=\int_{\Gamma} p_{l k}^{*} u_{k} d \Gamma-\frac{1}{2} u_{l}^{i}
$$

Após estas análises, pode-se retornar à expressão 2.76 e reescrevê-la da seguinte forma:

$$
c_{l k}^{i} u_{l}^{i}+\int_{\Gamma} p_{l k}^{*} u_{k} d \Gamma=\int_{\Gamma} u_{l k}^{*} p_{k} d \Gamma+\int_{\Omega} u_{l k}^{*} b_{k} d \Omega
$$

Cada integral da expressão 2.83 é definida como Valor Principal de Cauchy e, para o caso de $i$ se encontrar em um trecho suave de $\Gamma$, o termo $c_{l k}^{i}$ é igual à função Delta de Kronecker dividida por dois. Quando o ponto $i$ se encontra em um trecho de $\Gamma$ que não é suave, as integrais da equação 2.82 levam a diferentes valores de $c_{l k}^{i}$, tornando difícil encontrar uma expressão geral para este termo. No entanto, calcular este valor analiticamente não é necessário, pois ele pode ser obtido aplicando movimentos de corpo rígido ao sólido. Isto é explicado com mais detalhes na Seção 2.5.4.

Assim, por meio da resolução equação 2.83 se torna possível solucionar o problema de valor de contorno para o problema elástico tridimensional. 


\subsection{Formulação de elementos de contorno}

A equação 2.83 pode ser resolvida dividindo-se o contorno $\Gamma$ em elementos. Neste trabalho são utilizados elementos planos triangulares e contínuos, conforme mostrado na figura 2.7. Nesta figura, a superfície do elemento encontra-se representada por $\Gamma_{e}$. Em cada nó do elemento, estão definidos seis graus de liberdade. Como o sistema cartesiano é de três eixos, $x_{1}, x_{2}$ e $x_{3}$, cada nó tem três componentes de deslocamento $u$ e três componentes de força $p$, indicados na figura. Nestes valores, o índice subscrito indica a direção cartesiana e o índice sobrescrito indica a numeração local do nó.

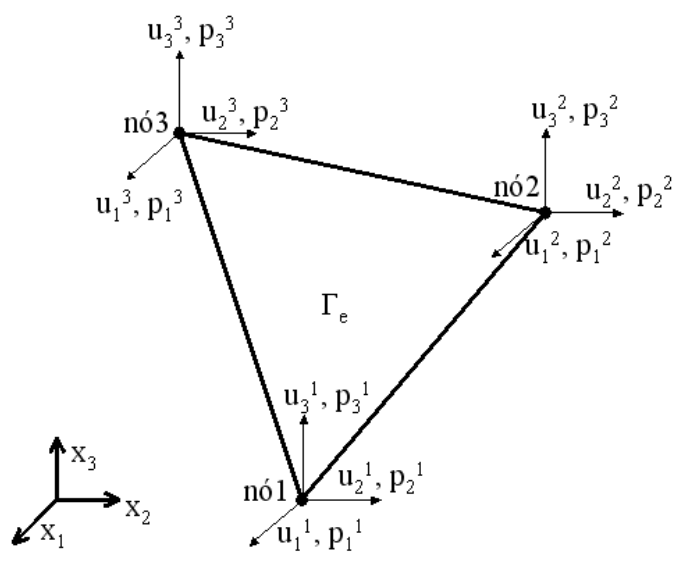

Figura 2.7: Elemento de contorno triangular

Ao longo de cada elemento deve ser definida uma função para representar os deslocamentos e as forças a partir de seus valores nodais. Ao escrever 2.83 para cada ponto nodal, é obtido um sistema de equações lineares, ao qual devem ser aplicadas as condições de contorno do problema. Então o sistema pode ser resolvido, obtendo-se todos os deslocamentos e forças incógnitos no contorno. Estes valores, em conjunto com as condições de contorno prescritas, constituem a solução do problema de valor de contorno.

Inicialmente, representa-se os deslocamentos $u$ e forças $p$ por funções conhecidas. Estas funções, contínuas ao longo de cada elemento $j$, podem ser escritas em forma matricial como:

$$
\begin{aligned}
& \mathbf{u}=\boldsymbol{\Phi} \mathbf{u}^{j} \\
& \mathbf{p}=\boldsymbol{\Phi} \mathbf{p}^{j}
\end{aligned}
$$

Os vetores $\mathbf{u}^{j}$ e $\mathbf{p}^{j}$ são, respectivamente, os deslocamentos e forças estabelecidos nos nós do elemento $j$. Para o caso tridimensional estes vetores têm $3 Q$ linhas, sendo $Q$ o 
número de nós por elemento. Os vetores $\mathbf{u}$ e $\mathbf{p}$ contêm, respectivamente, os deslocamentos e forças nas três direções em um ponto qualquer da superfície $\Gamma_{e}$ do elemento. Ou seja:

$$
\begin{gathered}
\mathbf{u}=\left\{\begin{array}{l}
u_{1} \\
u_{2} \\
u_{3}
\end{array}\right\} \\
\mathbf{p}=\left\{\begin{array}{l}
p_{1} \\
p_{2} \\
p_{3}
\end{array}\right\}
\end{gathered}
$$

A matriz de interpolação $\Phi$ tem dimensão $3 \times 3 Q$ e possui $Q$ funções $\phi$ diferentes. Para o elemento de contorno de três nós da figura 2.7, a matriz $\boldsymbol{\Phi}$ é:

$$
\boldsymbol{\Phi}=\left[\begin{array}{ccccccccc}
\phi_{1} & 0 & 0 & \phi_{2} & 0 & 0 & \phi_{3} & 0 & 0 \\
0 & \phi_{1} & 0 & 0 & \phi_{2} & 0 & 0 & \phi_{3} & 0 \\
0 & 0 & \phi_{1} & 0 & 0 & \phi_{2} & 0 & 0 & \phi_{3}
\end{array}\right]
$$

e o vetor $\mathbf{u}^{j}$ é:

$$
\left\{u_{j}\right\}=\left\{\begin{array}{lllllllll}
u_{1}^{1} & u_{2}^{1} & u_{3}^{1} & u_{1}^{2} & u_{2}^{2} & u_{3}^{2} & u_{1}^{3} & u_{2}^{3} & u_{3}^{3}
\end{array}\right\}
$$

Portanto, o deslocamento em um ponto qualquer de $\Gamma_{e}$ pode ser escrito como:

$$
\mathbf{u}=\left\{\begin{array}{c}
u_{1} \\
u_{2} \\
u_{3}
\end{array}\right\}=\left\{\begin{array}{c}
u_{1}^{1} \phi_{1}+u_{1}^{2} \phi_{2}+u_{1}^{3} \phi_{3} \\
u_{2}^{1} \phi_{1}+u_{2}^{2} \phi_{2}+u_{2}^{3} \phi_{3} \\
u_{3}^{1} \phi_{1}+u_{3}^{2} \phi_{2}+u_{3}^{3} \phi_{3}
\end{array}\right\}
$$

Pode-se observar, pela igualdade 2.90, que o deslocamento em uma direção de um ponto qualquer do elemento $j$ é função dos deslocamentos nodais do elemento. Observase também que as mesmas funções são utilizadas nas três direções.

A igualdade 2.90 também é válida para as forças, ou seja:

$$
\mathbf{p}=\left\{\begin{array}{c}
p_{1} \\
p_{2} \\
p_{3}
\end{array}\right\}=\left\{\begin{array}{c}
p_{1}^{1} \phi_{1}+p_{1}^{2} \phi_{2}+p_{1}^{3} \phi_{3} \\
p_{2}^{1} \phi_{1}+p_{2}^{2} \phi_{2}+p_{2}^{3} \phi_{3} \\
p_{3}^{1} \phi_{1}+p_{3}^{2} \phi_{2}+p_{3}^{3} \phi_{3}
\end{array}\right\}
$$


São adotadas funções $\phi$ lineares, conforme mostrado na figura 2.8 .
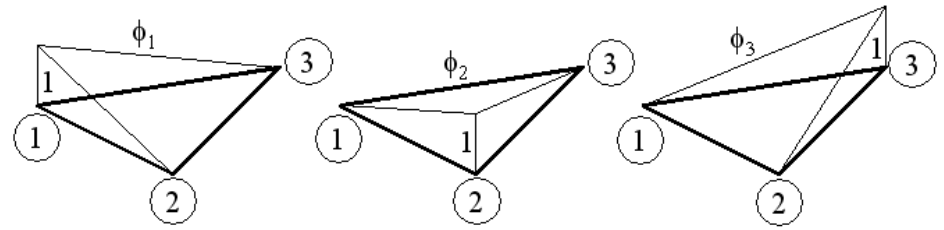

Figura 2.8: Funções interpoladoras adotadas

Para tornar a resolução das integrais mais simples, as funções $\phi_{1}, \phi_{2}$ e $\phi_{3}$ são definidas segundo um sistema de coordenadas local $\xi_{1} \xi_{2}$. Para informações mais detalhadas a respeito deste sistema homogêneo, recomenda-se consultar Assan (2003). Os sistemas local e global estão ilustrados na figura 2.9 .

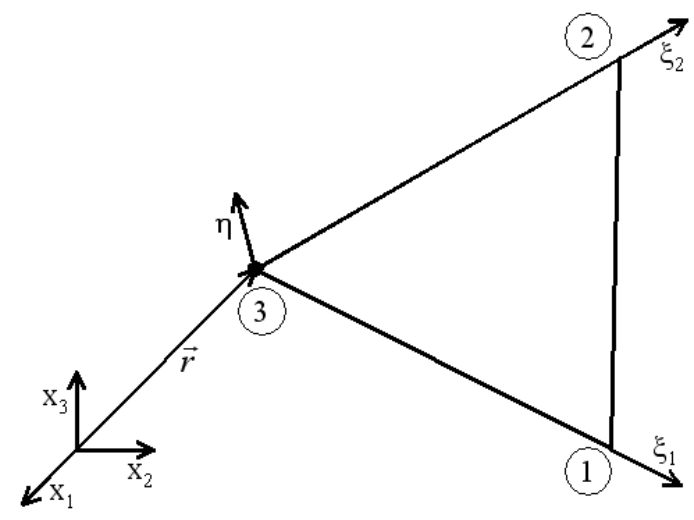

Figura 2.9: Sistemas de coordenadas global e local

Por serem lineares, as fuções $\phi_{1}, \phi_{2}$ e $\phi_{3}$ devem ser do tipo:

$$
\phi_{i}\left(\xi_{1}, \xi_{2}\right)=\alpha \xi_{1}+\beta \xi_{2}+\chi
$$

em que $\alpha, \beta$ e $\chi$ são constantes e $\xi_{1}$ e $\xi_{2}$ são coordenadas adimensionais. Uma opção para determinar estas constantes é obter equações a partir da figura 2.8. Assim:

$$
\begin{gathered}
\xi_{1}=0 \rightarrow \phi_{1}=1, \quad \phi_{2}=0, \quad \phi_{3}=0 \\
\xi_{2}=0 \rightarrow \phi_{1}=0, \quad \phi_{2}=1, \quad \phi_{3}=0 \\
\xi_{1}=0 \quad \text { e } \quad \xi_{2}=0 \rightarrow \phi_{1}=0, \quad \phi_{2}=0, \quad \phi_{3}=1
\end{gathered}
$$


A partir das equações 2.93, 2.94 e 2.95, pode-se concluir que:

$$
\begin{gathered}
\phi_{1}=\xi_{1} \\
\phi_{2}=\xi_{2} \\
\phi_{3}=-\xi_{1}-\xi_{2}+1
\end{gathered}
$$

Além dos deslocamentos $\mathbf{u}$ e das forças $\mathbf{p}$, também devem ser representadas de forma matricial as cargas volumétricas e as soluções fundamentais. As forças externas de domínio podem ser organizadas em um vetor que contenha suas componentes, ou seja:

$$
\mathbf{b}=\left\{\begin{array}{l}
b_{1} \\
b_{2} \\
b_{3}
\end{array}\right\}
$$

As soluções fundamentais podem ser representadas em forma matricial como segue:

$$
\begin{aligned}
& \mathbf{u}^{*}=\left[\begin{array}{ccc}
u_{11}^{*} & u_{12}^{*} & u_{13}^{*} \\
u_{21}^{*} & u_{22}^{*} & u_{23}^{*} \\
u_{31}^{*} & u_{32}^{*} & u_{33}^{*}
\end{array}\right] \\
& \mathbf{p}^{*}=\left[\begin{array}{lll}
p_{11}^{*} & p_{12}^{*} & p_{13}^{*} \\
p_{21}^{*} & p_{22}^{*} & p_{23}^{*} \\
p_{31}^{*} & p_{32}^{*} & p_{33}^{*}
\end{array}\right]
\end{aligned}
$$

Os coeficientes subscritos dos termos $u_{l k}^{*}$ e $p_{l k}^{*}$ das matrizes indicam a direção $l$ da carga unitária aplicada e a direção $k$ na qual o deslocamento ou força é considerado.

Aplicando a notação matricial apresentada, a equação 2.83 se torna:

$$
\mathbf{c}^{i} \mathbf{u}^{i}+\int_{\Gamma} \mathbf{p}^{*} \mathbf{u} d \Gamma=\int_{\Gamma} \mathbf{u}^{*} \mathbf{p} d \Gamma+\int_{\Omega} \mathbf{u}^{*} \mathbf{b} d \Omega
$$

Considerando agora que as funções de forma $\boldsymbol{\Phi}$ sejam substituídas na equação 2.102 . 
chega-se a uma equação onde são discriminadas as influências de cada elemento, isto é:

$$
\mathbf{c}^{i} \mathbf{u}^{i}+\sum_{j=1}^{n e}\left[\left(\int_{\Gamma_{j}} \mathbf{p}^{*} \boldsymbol{\Phi} d \Gamma\right) \mathbf{u}^{j}\right]=\sum_{j=1}^{n e}\left[\left(\int_{\Gamma_{j}} \mathbf{u}^{*} \boldsymbol{\Phi} d \Gamma\right) \mathbf{p}^{j}\right]
$$

em que ne é o número de elementos no contorno. Foi considerado na equação 2.103 que as forças externas de domínio, b, são nulas. É somada a influência da superfície $\Gamma_{j}$ de cada elemento de um até o número de elementos, ne. Os vetores $\mathbf{u}^{j}$ e $\mathbf{p}^{j}$ contêm, respectivamente, os deslocamentos e forças nos nós do elemento $j$.

\subsubsection{Transformação de coordenadas}

Para que seja possível integrar os elementos no sistema de coordenadas homogêneo $\xi_{1} \xi_{2}$, é preciso relacioná-lo ao sistema global $x_{1} x_{2} x_{3}$. Neste trabalho isto é feito conforme descrito em Assan (2003), ou seja, empregando-se as funções lineares 2.96, 2.97 e 2.98. Assim:

$$
\begin{aligned}
& x_{1}=x_{1}^{1} \phi_{1}+x_{1}^{2} \phi_{2}+x_{1}^{3} \phi_{3} \\
& x_{2}=x_{2}^{1} \phi_{1}+x_{2}^{2} \phi_{2}+x_{2}^{3} \phi_{3} \\
& x_{3}=x_{3}^{1} \phi_{1}+x_{3}^{2} \phi_{2}+x_{3}^{3} \phi_{3}
\end{aligned}
$$

Ou, em notação indicial:

$$
x_{i}=x_{i}^{j} \phi_{j}
$$

Portanto, a coordenada global $x_{i}$, correspondente à direção $i$, é escrita em 2.107 como o produto entre as funções lineares $\phi_{j}$ e as coordenadas $x_{i}^{j}$ dos vértices do elemento, sendo j o número do vértice.

Também é necessário analisar como as derivadas das coordenadas se relacionam para que o termo $d \Gamma$ das integrais possa ser substituído. Segundo Assan (2003), o sistema de coordenadas local pode ser relacionado ao global utilizando um Jacobiano de transformação, como mostrado abaixo:

$$
d \Gamma=d x_{1} d x_{2}=|\mathbf{J}| d \xi_{1} d \xi_{2}
$$


em que

$$
|\mathbf{J}|=\left|\begin{array}{cc}
\frac{\partial x_{1}}{\partial \xi_{1}} & \frac{\partial x_{1}}{\partial \xi_{2}} \\
\frac{\partial x_{2}}{\partial \xi_{1}} & \frac{\partial x_{2}}{\partial \xi_{2}}
\end{array}\right|=\frac{\partial x_{1}}{\partial \xi_{1}} \frac{\partial x_{2}}{\partial \xi_{2}}-\frac{\partial x_{1}}{\partial \xi_{2}} \frac{\partial x_{2}}{\partial \xi_{1}}
$$

Calcula-se então as derivadas da expressão 2.109, ou seja:

$$
\begin{aligned}
& \frac{\partial x_{1}}{\partial \xi_{1}}=\frac{\partial}{\partial \xi_{1}}\left(x_{1}^{1} \phi_{1}+x_{1}^{2} \phi_{2}+x_{1}^{3} \phi_{3}\right)=\frac{\partial}{\partial \xi_{1}}\left(x_{1}^{1} \xi_{1}+x_{1}^{2} \xi_{2}+x_{1}^{3}\left(-\xi_{1}-\xi_{2}+1\right)\right)=x_{1}^{1}-x_{1}^{3} \\
& \frac{\partial x_{1}}{\partial \xi_{2}}=\frac{\partial}{\partial \xi_{2}}\left(x_{1}^{1} \phi_{1}+x_{1}^{2} \phi_{2}+x_{1}^{3} \phi_{3}\right)=\frac{\partial}{\partial \xi_{2}}\left(x_{1}^{1} \xi_{1}+x_{1}^{2} \xi_{2}+x_{1}^{3}\left(-\xi_{1}-\xi_{2}+1\right)\right)=x_{1}^{2}-x_{1}^{3} \\
& \frac{\partial x_{2}}{\partial \xi_{1}}=\frac{\partial}{\partial \xi_{1}}\left(x_{2}^{1} \phi_{1}+x_{2}^{2} \phi_{2}+x_{2}^{3} \phi_{3}\right)=\frac{\partial}{\partial \xi_{1}}\left(x_{2}^{1} \xi_{1}+x_{2}^{2} \xi_{2}+x_{2}^{3}\left(-\xi_{1}-\xi_{2}+1\right)\right)=x_{2}^{1}-x_{2}^{3} \\
& \frac{\partial x_{2}}{\partial \xi_{2}}=\frac{\partial}{\partial \xi_{2}}\left(x_{2}^{1} \phi_{1}+x_{2}^{2} \phi_{2}+x_{2}^{3} \phi_{3}\right)=\frac{\partial}{\partial \xi_{2}}\left(x_{2}^{1} \xi_{1}+x_{2}^{2} \xi_{2}+x_{2}^{3}\left(-\xi_{1}-\xi_{2}+1\right)\right)=x_{2}^{2}-x_{2}^{3}
\end{aligned}
$$

Substitui-se então 2.110, 2.112, 2.111 e 2.113 em 2.109, obtendo:

$$
|\mathbf{J}|=\left(x_{1}^{1}-x_{1}^{3}\right)\left(x_{2}^{2}-x_{2}^{3}\right)-\left(x_{1}^{2}-x_{1}^{3}\right)\left(x_{2}^{1}-x_{2}^{3}\right)=2 A
$$

em que $A$ é a área do elemento.

Com o valor de $|\mathbf{J}|$ conhecido torna-se possível substituir a expressão 2.108 na equação 2.103 , obtendo:

$$
\mathbf{c}^{i} \mathbf{u}^{i}+\sum_{j=1}^{n e}\left[\left(\int_{\gamma} \mathbf{p}^{*} \boldsymbol{\Phi}|\mathbf{J}|^{j} d \xi_{1} d \xi_{2}\right) \mathbf{u}^{j}\right]=\sum_{j=1}^{n e}\left[\left(\int_{\gamma} \mathbf{u}^{*} \boldsymbol{\Phi}|\mathbf{J}|^{j} d \xi_{1} d \xi_{2}\right) \mathbf{p}^{j}\right]
$$

É importante observar que, após a transformação de coordenadas, é alterada a superfície dos elementos a serem integrados. A superfície $\Gamma_{j}$, definida no sistema global, é diferente para cada elemento $j$, enquanto que a superfície $\gamma$ é sempre restrita aos limites de zero a 1 do sistema homogêneo local.

As integrais da equação 2.115 são calculadas numericamente, empregando pontos de Hammer. Mais detalhes a respeito deste procedimento podem ser consultados em Assan (2003). O resultado é a expressão:

$$
\mathbf{c}^{i} \mathbf{u}^{i}+\sum_{j=1}^{n e}\left[|\mathbf{J}|^{j}\left\{\sum_{k=1}^{n h}\left(\mathbf{w}_{k} \mathbf{p}_{k}^{*} \boldsymbol{\Phi}_{k}\right)\right\} \mathbf{u}^{j}\right]=\sum_{j=1}^{n e}\left[|\mathbf{J}|^{j}\left\{\sum_{k=1}^{n h}\left(\mathbf{w}_{k} \mathbf{u}_{k}^{*} \boldsymbol{\Phi}_{k}\right)\right\} \mathbf{p}^{j}\right]
$$


O termo $n h$ se refere ao número de pontos de integração definidos sobre a superfície de cada elemento de contorno. As coordenadas $\xi_{1}$ e $\xi_{2}$ bem como o peso $\mathbf{w}_{k}$ de cada ponto podem ser encontrados tabelados na literatura, citando-se por exemplo Assan (2003).

\subsubsection{Tratamento da singularidade}

Caso o ponto fonte esteja localizado em um dos vértices do elemento integrado, as integrais da equação 2.115 apresentam problemas de singularidade. Por causa destes problemas, a técnica de integração apresentada na Seção anterior se torna imprecisa. Apresenta-se então nesta Seção uma forma eficiente de integrar este elemento, a qual foi desenvolvida no trabalho de Guiggiani e Gigante (1990) e empregada por diversos outros autores. Esta técnica permite que a singularidade seja eliminada, possibilitando o cálculo das integrais de forma precisa.

Considera-se um elemento $j$ qualquer a ser integrado, e que o ponto fonte esteja localizado em seu domínio $\Gamma_{j}$. Como neste trabalho são empregados elementos contínuos, o ponto fonte coincide com um dos três vértices do triângulo. A figura 2.10 ilustra o problema.

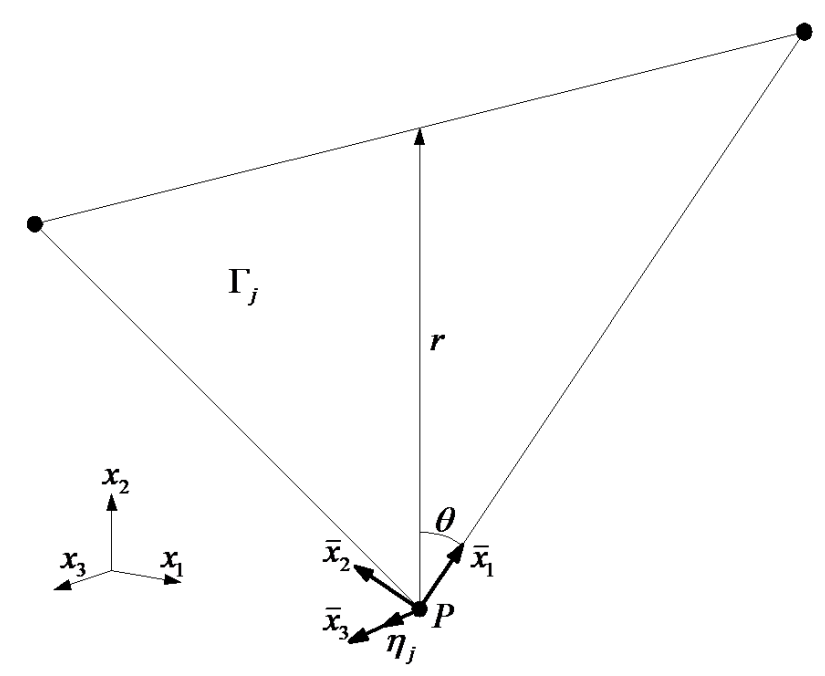

Figura 2.10: Ponto fonte em um dos vértices do elemento

Na figura, o ponto fonte está denominado por $P$. Aparecem dois novos sistemas de coordenadas além do sistema global $x_{1} x_{2} x_{3}$, o sistema $\bar{x}_{1} \bar{x}_{2} \bar{x}_{3}$ e o sistema $r \theta$. O sistema $\bar{x}_{1} \bar{x}_{2} \bar{x}_{3}$ tem origem em $\mathrm{P}$, também é retangular e seu plano $\bar{x}_{1} \bar{x}_{2}$ contém o elemento. $\mathrm{O}$ eixo $\bar{x}_{1}$ passa pelo nó seguinte ao da origem, seguindo a orientação positiva da numeração local do elemento, e o eixo $\bar{x}_{3}$ é perpendicular ao plano do elemento, tendo mesma direção 
e sentido que o vetor normal $\eta_{j}$. O sistema $r \theta$, por sua vez, é de coordenadas polares, sendo $r$ variável de zero à distância entre $P$ e o perímetro do triângulo e $\theta$ o ângulo entre os eixos $r$ e $\bar{x}_{1}$.

A estratégia é integrar o elemento empregando o sistema $r \theta$, sendo portanto necessário transformar as coordenadas. No item 2.5.1 foi demonstrado como para passar do sistema $x_{1} x_{2} x_{3}$ ao sistema $\xi_{1} \xi_{2}$, entretanto este procedimento foi simplificado pelo fato de que as funções de forma $\phi_{1}, \phi_{2}$ e $\phi_{3}$ presentes na matriz $\boldsymbol{\Phi}$ da equação 2.103 foram escritas diretamente no sistema $\xi_{1} \xi_{2}$. No presente caso, como as funções $\phi_{1}, \phi_{2}$ e $\phi_{3}$ também devem estar no sistema $r \theta$ para que possam ser integradas, é necessário passá-las de $\xi_{1} \xi_{2}$ para $r \theta$. Este procedimento, assim como em Barbirato (1999) e Almeida (2003b), é aqui dividido em duas etapas. A primeira é passar do sistema $\xi_{1} \xi_{2}$ ao sistema $\bar{x}_{1} \bar{x}_{2} \bar{x}_{3}$ e a segunda é ir de $\bar{x}_{1} \bar{x}_{2} \bar{x}_{3}$ para $r \theta$.

O ponto de partida é escrever as funções de forma em função das coordenadas retangulares $\bar{x}_{1} \bar{x}_{2} \bar{x}_{3}$. Estas expressões são apresentadas em Assan (2003). Assim:

$$
\begin{array}{r}
\phi_{1}=\xi_{1}=\frac{1}{2 A}\left[\bar{x}_{1}^{2} \bar{x}_{2}^{3}-\bar{x}_{1}^{3} \bar{x}_{2}^{2}+\bar{x}_{1}\left(\bar{x}_{2}^{2}-\bar{x}_{2}^{3}\right)+\bar{x}_{2}\left(\bar{x}_{1}^{3}-\bar{x}_{1}^{2}\right)\right] \\
\phi_{2}=\xi_{2}=\frac{1}{2 A}\left[\bar{x}_{1}^{3} \bar{x}_{2}^{1}-\bar{x}_{1}^{1} \bar{x}_{2}^{3}+\bar{x}_{1}\left(\bar{x}_{2}^{3}-\bar{x}_{2}^{1}\right)+\bar{x}_{2}\left(\bar{x}_{1}^{1}-\bar{x}_{1}^{3}\right)\right] \\
\phi_{3}=1-\xi_{1}-\xi_{2}=\frac{1}{2 A}\left[\bar{x}_{1}^{1} \bar{x}_{2}^{2}-\bar{x}_{1}^{2} \bar{x}_{2}^{1}+\bar{x}_{1}\left(\bar{x}_{2}^{1}-\bar{x}_{2}^{2}\right)+\bar{x}_{2}\left(\bar{x}_{1}^{2}-\bar{x}_{1}^{1}\right)\right]
\end{array}
$$

ou, em notação indicial:

$$
\phi_{i}=\frac{1}{2 A}\left[\bar{x}_{1}^{j} \bar{x}_{2}^{k}-\bar{x}_{1}^{k} \bar{x}_{2}^{j}+\bar{x}_{1}\left(\bar{x}_{2}^{j}-\bar{x}_{2}^{k}\right)+\bar{x}_{2}\left(\bar{x}_{1}^{k}-\bar{x}_{1}^{j}\right)\right]
$$

Na expressão 2.120 os índices subscritos indicam a direção cartesiana e os sobrescritos a numeração local do nó no elemento. As coordenadas dos vértices no sistema local $\bar{x}_{1} \bar{x}_{2} \bar{x}_{3}$ podem ser determinadas a partir de suas coordenadas globais $x_{1} x_{2} x_{3}$ e da matriz de rotação do elemento. Para maiores detalhes, recomenda-se consultar Oñate (1995).

Como a equação integral 2.115 é escrita para o sistema de coordenadas $\xi_{1} \xi_{2}$, parte-se dela para a obtenção das integrais em $\bar{x}_{1} \bar{x}_{2} \bar{x}_{3}$. É importante salientar que, neste caso, o objeto de análise não são todos os elementos somados na equação 2.115, mas somente aquele ao qual pertence o ponto fonte. Por este motivo, extrai-se de 2.115 as integrais 
referentes a este elemento:

$$
\begin{aligned}
& \mathbf{I}_{1}=\int_{\gamma_{P}} \mathbf{p}^{*} \boldsymbol{\Phi}|\mathbf{J}| d \xi_{1} d \xi_{2} \\
& \mathbf{I}_{2}=\int_{\gamma_{P}} \mathbf{u}^{*} \boldsymbol{\Phi}|\mathbf{J}| d \xi_{1} d \xi_{2}
\end{aligned}
$$

O índice subscrito $P$ indica que o ponto fonte pertence ao elemento a ser integrado. Seguindo as deduções, como as expressões das funções de forma já foram determinadas no sistema $\bar{x}_{1} \bar{x}_{2} \bar{x}_{3}$, basta calcular o Jacobiano $\left|\mathbf{J}_{1}\right|$ desta transformação de coordenadas para que se possa realizar a seguinte substituição nas integrais 2.121 e 2.122 .

$$
|\mathbf{J}| d \xi_{1} d \xi_{2}=d \Gamma=\left|\mathbf{J}_{1}\right||\mathbf{J}| d \bar{x}_{1} d \bar{x}_{2}
$$

Segue, portanto, o cálculo de $\left|\mathbf{J}_{1}\right|$ :

$$
\left|\mathbf{J}_{1}\right|=\left|\begin{array}{ll}
\frac{\partial \xi_{1}}{\partial \bar{x}_{1}} & \frac{\partial \xi_{1}}{\partial \bar{x}_{2}} \\
\frac{\partial \xi_{2}}{\partial \bar{x}_{1}} & \frac{\partial \xi_{2}}{\partial \bar{x}_{2}}
\end{array}\right|=\frac{\partial \xi_{1}}{\partial \bar{x}_{1}} \frac{\partial \xi_{2}}{\partial \bar{x}_{2}}-\frac{\partial \xi_{1}}{\partial \bar{x}_{2}} \frac{\partial \xi_{2}}{\partial \bar{x}_{1}}
$$

Calcula-se as derivadas da expressão 2.124 a partir das expressões 2.117 e 2.118. Assim:

$$
\begin{aligned}
& \frac{\partial \xi_{1}}{\partial \bar{x}_{1}}=\frac{1}{2 A}\left[\bar{x}_{2}^{2}-\bar{x}_{2}^{3}\right] \\
& \frac{\partial \xi_{1}}{\partial \bar{x}_{2}}=\frac{1}{2 A}\left[\bar{x}_{1}^{3}-\bar{x}_{1}^{2}\right] \\
& \frac{\partial \xi_{2}}{\partial \bar{x}_{1}}=\frac{1}{2 A}\left[\bar{x}_{2}^{3}-\bar{x}_{2}^{1}\right] \\
& \frac{\partial \xi_{2}}{\partial \bar{x}_{2}}=\frac{1}{2 A}\left[\bar{x}_{1}^{1}-\bar{x}_{1}^{3}\right]
\end{aligned}
$$

Substituindo 2.125, 2.126, 2.127 e 2.128 em 2.124, obtém-se:

$$
\begin{gathered}
\left|\mathbf{J}_{1}\right|=\left(\frac{1}{2 A}\left[\bar{x}_{2}^{2}-\bar{x}_{2}^{3}\right]\right)\left(\frac{1}{2 A}\left[\bar{x}_{1}^{1}-\bar{x}_{1}^{3}\right]\right)-\left(\frac{1}{2 A}\left[\bar{x}_{1}^{3}-\bar{x}_{1}^{2}\right]\right)\left(\frac{1}{2 A}\left[\bar{x}_{2}^{3}-\bar{x}_{2}^{1}\right]\right) \\
\left|\mathbf{J}_{1}\right|=\frac{1}{4 A^{2}}\left[\left(\bar{x}_{2}^{2}-\bar{x}_{2}^{3}\right)\left(\bar{x}_{1}^{1}-\bar{x}_{1}^{3}\right)-\left(\bar{x}_{2}^{3}-\bar{x}_{2}^{1}\right)\left(\bar{x}_{1}^{3}-\bar{x}_{1}^{2}\right)\right]
\end{gathered}
$$

Por analogia à expressão 2.114, conclui-se que o termo entre colchetes é igual a duas 
vezes a área do elemento. Portanto:

$$
\left|\mathbf{J}_{1}\right|=\frac{1}{4 A^{2}}[2 A]=\frac{1}{2 A}
$$

Assim, substituindo a expressão 2.131 em 2.123 .

$$
|\mathbf{J}| d \xi_{1} d \xi_{2}=d \Gamma=\frac{1}{2 A} 2 A d \bar{x}_{1} d \bar{x}_{2}=d \bar{x}_{1} d \bar{x}_{2}
$$

Encerrando a primeira etapa da transformação de coordenadas, substitui-se a equação 2.132 nas integrais 2.121 e 2.122 . O resultado é:

$$
\begin{aligned}
& \mathbf{I}_{1}=\int_{\Gamma_{P}} \mathbf{p}^{*} \boldsymbol{\Phi} d \bar{x}_{1} d \bar{x}_{2} \\
& \mathbf{I}_{2}=\int_{\Gamma_{P}} \mathbf{u}^{*} \boldsymbol{\Phi} d \bar{x}_{1} d \bar{x}_{2}
\end{aligned}
$$

Observa-se que a superfície integrada não é mais $\gamma_{P}$, definida no sistema local oblíquo, e sim $\Gamma_{P}$, que é definida no sistema local retangular.

A segunda etapa é passar do sistema de coordenadas retangulares $\bar{x}_{1} \bar{x}_{2} \bar{x}_{3}$ ao sistema de coordenadas polares $r \theta$. O sistema $\bar{x}_{1} \bar{x}_{2} \bar{x}_{3}$ pode ser relacionado com $r \theta$ a partir da projeção do vetor $r$ nos eixos $\bar{x}_{1}$ e $\bar{x}_{2}$, ou seja:

$$
\begin{aligned}
& \bar{x}_{1}=r \cos \theta \\
& \bar{x}_{2}=r \operatorname{sen} \theta
\end{aligned}
$$

É necessário então reescrever as funções de forma $\phi_{1}, \phi_{2}$ e $\phi_{3}$ substituindo-se as igualdades 2.135 e 2.136 nas expressões 2.117, 2.118 e 2.119, Assim:

$$
\begin{aligned}
& \phi_{1}=\frac{1}{2 A}\left[\bar{x}_{1}^{2} \bar{x}_{2}^{3}-\bar{x}_{1}^{3} \bar{x}_{2}^{2}+r \cos \theta\left(\bar{x}_{2}^{2}-\bar{x}_{2}^{3}\right)+r \operatorname{sen} \theta\left(\bar{x}_{1}^{3}-\bar{x}_{1}^{2}\right)\right] \\
& \phi_{2}=\frac{1}{2 A}\left[\bar{x}_{1}^{3} \bar{x}_{2}^{1}-\bar{x}_{1}^{1} \bar{x}_{2}^{3}+r \cos \theta\left(\bar{x}_{2}^{3}-\bar{x}_{2}^{1}\right)+r \operatorname{sen} \theta\left(\bar{x}_{1}^{1}-\bar{x}_{1}^{3}\right)\right] \\
& \phi_{3}=\frac{1}{2 A}\left[\bar{x}_{1}^{1} \bar{x}_{2}^{2}-\bar{x}_{1}^{2} \bar{x}_{2}^{1}+r \cos \theta\left(\bar{x}_{2}^{1}-\bar{x}_{2}^{2}\right)+r \operatorname{sen} \theta\left(\bar{x}_{1}^{2}-\bar{x}_{1}^{1}\right)\right]
\end{aligned}
$$


ou, em notação indicial:

$$
\phi_{i}=\frac{1}{2 A}\left[\bar{x}_{1}^{j} \bar{x}_{2}^{k}-\bar{x}_{1}^{k} \bar{x}_{2}^{j}+r \cos \theta\left(\bar{x}_{2}^{j}-\bar{x}_{2}^{k}\right)+r \operatorname{sen} \theta\left(\bar{x}_{1}^{k}-\bar{x}_{1}^{j}\right)\right]
$$

Para tornar a utilização desta expressão mais prática, ela é reescrita como:

$$
\phi_{i}=\frac{1}{2 A}\left[\alpha_{i}+r \beta_{i}\right]
$$

com

$$
\begin{gathered}
\alpha_{i}=\bar{x}_{1}^{j} \bar{x}_{2}^{k}-\bar{x}_{1}^{k} \bar{x}_{2}^{j} \\
\beta_{i}=\cos \theta\left(\bar{x}_{2}^{j}-\bar{x}_{2}^{k}\right)+\operatorname{sen} \theta\left(\bar{x}_{1}^{k}-\bar{x}_{1}^{j}\right)
\end{gathered}
$$

Para relacionar as derivadas das coordenadas emprega-se novamente um Jacobiano de transformação. Ou seja:

$$
d \bar{x}_{1} d \bar{x}_{2}=d \Gamma=\left|\mathbf{J}_{2}\right| d r d \theta
$$

Segue-se então para o cálculo de $\left|\mathbf{J}_{2}\right|$ :

$$
\left|\mathbf{J}_{2}\right|=\left|\begin{array}{cc}
\frac{\partial \bar{x}_{1}}{\partial r} & \frac{\partial \bar{x}_{1}}{\partial \theta} \\
\frac{\partial \bar{x}_{2}}{\partial r} & \frac{\partial \bar{x}_{2}}{\partial \theta}
\end{array}\right|=\frac{\partial \bar{x}_{1}}{\partial r} \frac{\partial \bar{x}_{2}}{\partial \theta}-\frac{\partial \bar{x}_{1}}{\partial \theta} \frac{\partial \bar{x}_{2}}{\partial r}
$$

com

$$
\begin{gathered}
\frac{\partial \bar{x}_{1}}{\partial r}=\cos \theta \\
\frac{\partial \bar{x}_{1}}{\partial \theta}=-r \operatorname{sen} \theta \\
\frac{\partial \bar{x}_{2}}{\partial r}=\operatorname{sen} \theta \\
\frac{\partial \bar{x}_{2}}{\partial \theta}=r \cos \theta
\end{gathered}
$$

Substituindo 2.146, 2.147, 2.148 e 2.149 em 2.145, obtém-se:

$$
\left|\mathbf{J}_{2}\right|=r(\cos \theta)^{2}+r(\operatorname{sen} \theta)^{2}=r
$$

Conhecido o valor de $\left|\mathbf{J}_{2}\right|$ e as funções de forma no sistema polar $r \theta$, o passo seguinte 
é a substituição da expressão 2.144 nas integrais 2.133 e 2.134. Portanto:

$$
\begin{aligned}
& \mathbf{I}_{1}=\int_{0}^{2 \pi} \int_{0}^{R} \mathbf{p}^{*} \boldsymbol{\Phi}\left|\mathbf{J}_{2}\right| d r d \theta \\
& \mathbf{I}_{2}=\int_{0}^{2 \pi} \int_{0}^{R} \mathbf{u}^{*} \boldsymbol{\Phi}\left|\mathbf{J}_{2}\right| d r d \theta
\end{aligned}
$$

Nas expressões, os limites de integração indicam a faixa percorrida por cada variável a ser integrada. A faixa de $r$ vai de zero até $R$, que é a distância de $P$ até o perímetro do triângulo, enquanto a faixa de $\theta$ vai de zero a $2 \pi$. Observa-se, pela figura 2.10 , que seria suficiente variar $\theta$ de zero até o ângulo interno do vértice de $P$ para percorrer toda a superfície de cada elemento. Entretanto, escreve-se a equação para o caso mais geral no qual $P$ pode estar em qualquer posição da superfície a ser integrada. Isto permite que um termo singular possa ser posteriormente eliminado.

Resta então calcular as integrais 2.151 e 2.152. Como será visto mais adiante, torna-se possível que a parcela singular das integrais seja igualada a zero. Segundo Guiggiani e Gigante (1990), isto viabiliza que estas integrais sejam calculadas numericamente sem prejudicar a precisão dos resultados. Entretanto, para reduzir o tempo de processamento, neste trabalho adota-se o mesmo procedimento empregado em Almeida (2003b) e Barbirato (1999), denominado integração semi-analítica. Recebe este nome porque a integral em $r$ é feita analiticamente, enquanto a integral em $\theta$ é feita numericamente.

Para calcular as integrais 2.151 e 2.152 analiticamente em $r$ é necessário efetuar os produtos das matrizes e analisar o que acontece com cada termo resultante. Inicialmente, reescreve-se as soluções fundamentais em notação indicial:

$$
\begin{gathered}
u_{l k}^{*}=\frac{1}{16 \pi \mu(1-\nu) r}\left[(3-4 \nu) \delta_{l k}+r_{, l} r_{, k}\right] \\
p_{l k}^{*}=\frac{-1}{8 \pi(1-\nu) r^{2}}\left[r_{, \eta}\left[(1-2 \nu) \delta_{l k}+3 r_{, l} r_{, k}\right]+(1-2 \nu)\left(\eta_{l} r_{, k}-\eta_{k} r_{, l}\right)\right]
\end{gathered}
$$

É possível combinar a estas expressões o termo $\left|\mathbf{J}_{2}\right|$, que aparece multiplicando-as nas integrais 2.151 e 2.152 e é dado pela igualdade 2.150. Assim:

$$
U_{l k}^{*}=u_{l k}^{*}\left|\mathbf{J}_{2}\right|=\frac{1}{16 \pi \mu(1-\nu)}\left[(3-4 \nu) \delta_{l k}+r_{, l} r_{, k}\right]
$$




$$
P_{l k}^{*}=p_{l k}^{*}\left|\mathbf{J}_{2}\right|=\frac{-1}{8 \pi(1-\nu) r}\left[r_{, \eta}\left[(1-2 \nu) \delta_{l k}+3 r_{, l} r_{, k}\right]+(1-2 \nu)\left(\eta_{l} r_{, k}-\eta_{k} r_{, l}\right)\right]
$$

A expressão 2.156 pode ser simplificada, pois o termo $r_{, \eta}$, correspondente à projeção de $r$ na direção de $\eta$, é igual a zero no caso em questão. Isto acontece porque a integral semianalítica é empregada somente quando o ponto fonte pertence ao domínio do elemento integrado, e neste caso o versor normal $\eta$ é sempre perpendicular a $r$. Desta forma, a expressão 2.156 pode ser reescrita como:

$$
P_{l k}^{*}=\frac{-1}{8 \pi(1-\nu) r}(1-2 \nu)\left(\eta_{l} r_{, k}-\eta_{k} r_{, l}\right)
$$

O próximo passo é fazer o produto das expressões 2.155 e 2.157 pela matriz de funções de forma $\boldsymbol{\Phi}$, representadas no sistema de coordenadas polares $r \theta$ pela expressão 2.141. Assim:

$$
\begin{gathered}
m_{l k i}=U_{l k}^{*} \phi_{i}=\frac{\left[(3-4 \nu) \delta_{l k}+r_{, l} r_{, k}\right]}{32 A \pi \mu(1-\nu)}\left[\alpha_{i}+r \beta_{i}\right] \\
n_{l k i}=P_{l k}^{*} \phi_{i}=\frac{(2 \nu-1)\left(\eta_{l} r_{, k}-\eta_{k} r_{, l}\right)}{16 A \pi(1-\nu)}\left[\frac{\alpha_{i}}{r}+\beta_{i}\right]
\end{gathered}
$$

Observa-se que $m_{l k i}$ é uma função linear em relação a $r$, portanto sua integral pode ser efetuada sem problemas:

$$
M_{l k i}=\int_{0}^{R} \frac{\left[(3-4 \nu) \delta_{l k}+r_{, l} r_{, k}\right]}{32 A \pi \mu(1-\nu)}\left[\alpha_{i}+r \beta_{i}\right] d r=\frac{\left[(3-4 \nu) \delta_{l k}+r_{, l} r_{, k}\right]}{32 A \pi \mu(1-\nu)}\left[R \alpha_{i}+\frac{R^{2}}{2} \beta_{i}\right]
$$

A função $n_{l k i}$, por sua vez, possui termos do tipo $1 / r$, e sua integral é singular em $r=0$. No entanto, segundo Barbirato (1999), como o ângulo $\theta$ varia de zero a $2 \pi$, a parcela singular desta integral pode ser igualada a zero. Para melhores esclarecimentos a respeito da eliminação da sigularidade, recomenda-se consultar o trabalho de Guiggiani e Gigante (1990).

Seguindo a dedução, após igualar o termo singular a zero, obtém-se:

$$
N_{l k i}=\int_{0}^{R} \frac{(2 \nu-1)\left(\eta_{l} r_{, k}-\eta_{k} r_{, l}\right)}{16 A \pi(1-\nu)}\left[\frac{\alpha_{i}}{r}+\beta_{i}\right] d r=\frac{(2 \nu-1)\left(\eta_{l} r_{, k}-\eta_{k} r_{, l}\right)}{16 A \pi(1-\nu)}\left[\alpha_{i} \ln (R)+\beta_{i} R\right]
$$

Caso se queira empregar as expressões 2.160 e 2.161 matricialmente, os termos das 
matrizes podem ser organizados da seguinte maneira:

$$
\begin{gathered}
\mathbf{M}=\left[\begin{array}{llllllllll}
m_{111} & m_{121} & m_{131} & m_{112} & m_{122} & m_{132} & m_{113} & m_{123} & m_{133} \\
m_{211} & m_{221} & m_{231} & m_{212} & m_{222} & m_{232} & m_{213} & m_{223} & m_{233} \\
m_{311} & m_{321} & m_{331} & m_{312} & m_{322} & m_{332} & m_{313} & m_{323} & m_{333}
\end{array}\right] \\
\mathbf{N}=\left[\begin{array}{llllllllll}
n_{111} & n_{121} & n_{131} & n_{112} & n_{122} & n_{132} & n_{113} & n_{123} & n_{133} \\
n_{211} & n_{221} & n_{231} & n_{212} & n_{222} & n_{232} & n_{213} & n_{223} & n_{233} \\
n_{311} & n_{321} & n_{331} & n_{312} & n_{322} & n_{332} & n_{313} & n_{323} & n_{333}
\end{array}\right]
\end{gathered}
$$

Após estas deduções, chega-se às seguintes igualdades:

$$
\begin{aligned}
& \mathbf{I}_{1}=\int_{0}^{2 \pi} \mathbf{N} d \theta \\
& \mathbf{I}_{2}=\int_{0}^{2 \pi} \mathbf{M} d \theta
\end{aligned}
$$

As integrais 2.164 e 2.165 são então efetuadas numericamente. Para simplificar este procedimento a coordenada $\theta$ é transformada em uma coordenada adimensional $\zeta$, definida em cada um dos três lados do elemento. A figura 2.11 ilustra o novo sistema de coordenadas.

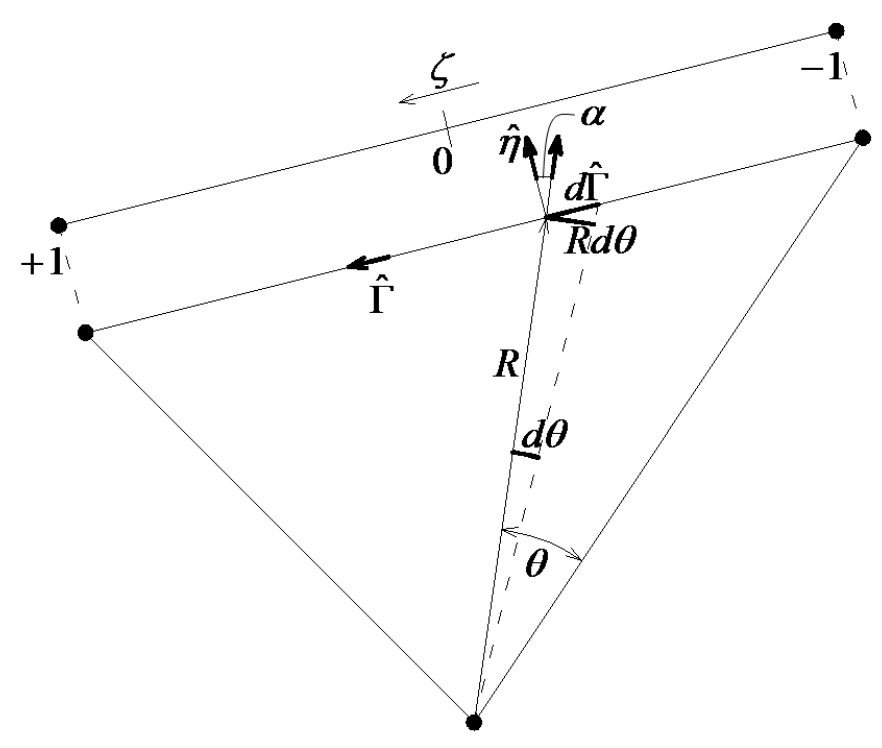

Figura 2.11: Coordenada adimensional $\zeta$

A coordenada $\zeta$ varia de -1 a +1 ao longo do comprimento $L$ do lado em questão. No perímetro do triângulo é definido um contorno fictício $\hat{\Gamma}$ e um versor normal $\hat{\eta}$, e o 
ângulo entre $R$ e $\hat{\eta}$ é denotado por $\alpha$. Dois segmentos de reta estão em destaque, um correspondente a $d \hat{\Gamma}$ e outro correspondente a $R d \theta$. Estes segmentos são necessários para demonstrar, posteriormente, a relação entre $d \theta$ e $d \hat{\Gamma}$.

Uma forma conveniente de passar de $\theta$ para $\zeta$ é primeiramente retornar ao sistema de coordenadas $\bar{x}_{1} \bar{x}_{2} \bar{x}_{3}$ por meio das relações 2.135 e 2.136 e então passar de $\bar{x}_{1} \bar{x}_{2} \bar{x}_{3}$ para $\zeta$. A primeira transformação altera o termo $\beta_{i}$ presente nas expressões 2.160 e 2.161, sendo necessário efetuar a seguinte substituição:

$$
R \beta_{i}=R \cos \theta\left(\bar{x}_{2}^{j}-\bar{x}_{2}^{k}\right)+R \operatorname{sen} \theta\left(\bar{x}_{1}^{k}-\bar{x}_{1}^{j}\right)=\bar{x}_{1}\left(\bar{x}_{2}^{j}-\bar{x}_{2}^{k}\right)+\bar{x}_{2}\left(\bar{x}_{1}^{k}-\bar{x}_{1}^{j}\right)=\gamma_{i}
$$

Assim, substituindo $R \beta_{i}$ por $\gamma_{i}$ nas expressões 2.160 e 2.161, obtém-se:

$$
\begin{gathered}
\hat{M}_{l k i}=\frac{\left[(3-4 \nu) \delta_{l k}+r_{, l} r_{, k}\right]}{32 A \pi \mu(1-\nu)} R\left[\alpha_{i}+\frac{1}{2} \gamma_{i}\right] \\
\hat{N}_{l k i}=\frac{(2 \nu-1)\left(\eta_{l} r_{, k}-\eta_{k} r_{, l}\right)}{16 A \pi(1-\nu)}\left[\alpha_{i} \ln (R)+\gamma_{i}\right]
\end{gathered}
$$

O Jacobiano da transformação de $\theta$ para $\bar{x}_{1} \bar{x}_{2} \bar{x}_{3}$ pode ser obtido geometricamente a partir da figura 2.11. A projeção de $R$ na direção do versor normal $\hat{\eta}$ corresponde ao co-seno do ângulo $\alpha$ indicado na figura, e é por definição também igual à derivada de $R$ em relação a $\hat{\eta}$. Portanto:

$$
\cos \alpha=\frac{d R}{d \hat{\eta}}
$$

É possível observar na figura que o ângulo entre os dois segmentos em destaque, $d \hat{\Gamma}$ e $R d \theta$, é $\alpha$. Assim:

$$
\cos \alpha=\frac{R d \theta}{d \hat{\Gamma}}
$$

Ao substituir as relações 2.169 e 2.170 uma na outra, chega-se às relações:

$$
\frac{d R}{d \hat{\eta}}=\frac{R d \theta}{d \hat{\Gamma}} \rightarrow d \theta=\frac{1}{R} \frac{d R}{d \hat{\eta}} d \hat{\Gamma}
$$

Desta forma, as integrais 2.164 e 2.165 se tornam:

$$
\mathbf{I}_{1}=\int_{0}^{2 \pi} \hat{\mathbf{N}} \frac{1}{R} \frac{d R}{d \hat{\eta}} d \hat{\Gamma}
$$




$$
\mathbf{I}_{2}=\int_{0}^{2 \pi} \hat{\mathbf{M}} \frac{1}{R} \frac{d R}{d \hat{\eta}} d \hat{\Gamma}
$$

em que as matrizes $\hat{\mathbf{N}}$ e $\hat{\mathbf{M}}$ são representadas, em notação indicial, pelas expressões 2.168 e 2.167, respectivamente. O cálculo de $d R / d \hat{\eta}$ pode ser feito com uma expressão análoga à igualdade 2.55, a qual é empregada na Seção 2.3 para a determinação de $r_{, \eta}$. Assim:

$$
\frac{d R}{d \hat{\eta}}=\frac{d R}{d \bar{x}_{i}} \hat{\eta}_{i}
$$

com

$$
\frac{d R}{d \bar{x}_{i}}=\frac{R_{i}}{R}
$$

e

$$
R_{i}=\left|\bar{x}_{i}(S)-\bar{x}_{i}(P)\right|
$$

Antes de prosseguir com as deduções, é importante observar que a presença do termo $d R / d \hat{\eta}$ nas integrais permite que as contribuições dos dois lados adjacentes ao ponto $P$ sejam igualadas a zero. Isto acontece porque o versor $\hat{\eta}$ é perpendicular a $R$ ao longo de todo o comprimento destes lados. Como o termo $d R / d \hat{\eta}$ corresponde à projeção de $R$ em $\hat{\eta}$, ele pode ser igualado a zero assim como a contribuição desses lados. Isto torna esta formulação ainda mais vantajosa, pois a integral de superfície se reduz a uma integral ao longo de um único segmento de reta.

O passo final antes de integrar as expressões é relacionar $d \hat{\Gamma}$ com $d \zeta$. Isto pode ser feito relacionando $\hat{\Gamma} \operatorname{com} \zeta$ para posteriormente calcular o Jacobiano desta transformação. A figura 2.12 ilustra estes dois sistemas:

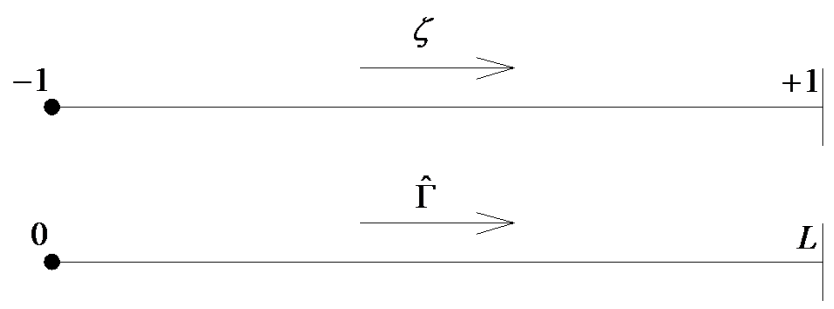

Figura 2.12: Eixos $\hat{\Gamma}$ e $\zeta$

Com o auxílio da figura 2.12 , nota-se que quando $\hat{\Gamma}$ é zero $\zeta$ é igual a -1 e que quando $\hat{\Gamma}$ é igual a $L$ a variável $\zeta$ é +1 . A partir destas relações conclui-se que:

$$
\hat{\Gamma}=\frac{L}{2}(1+\zeta)
$$


Desta forma, escreve-se a relação:

$$
d \hat{\Gamma}=\left|\mathbf{J}_{3}\right| d \zeta
$$

em que o Jacobiano é simplesmente:

$$
\left|\mathbf{J}_{3}\right|=\frac{d \hat{\Gamma}}{d \zeta}=\frac{L}{2}
$$

Para a integração numérica emprega-se a Quadratura de Gauss-Legendre, a qual pode ser vista com mais detalhes em Assan (2003). Assim, as integrais 2.172 e 2.173 são reescritas da seguinte forma:

$$
\begin{gathered}
I_{l k i 1}=\frac{L}{2} \sum_{m=1}^{n g}\left[\frac{(2 \nu-1)\left(\eta_{l} r_{, k}-\eta_{k} r_{, l}\right)}{16 A \pi(1-\nu) R}\left[\alpha_{i} \ln (R)+\gamma_{i}\right] \frac{d R}{d \hat{\eta}} w_{m}\right] \\
I_{l k i 2}=\frac{L}{2} \sum_{m=1}^{n g}\left[\frac{\left[(3-4 \nu) \delta_{l k}+r_{, l} r_{, k}\right]}{32 A \pi \mu(1-\nu)}\left[\alpha_{i}+\frac{1}{2} \gamma_{i}\right] \frac{d R}{d \hat{\eta}} w_{m}\right]
\end{gathered}
$$

O somatório vai de 1 até o número de pontos de Gauss $n g$, sendo $w_{m}$ o peso de cada ponto. Estando o ponto campo em uma posição correspondente a um valor $\zeta$ qualquer, é possível determinar suas coordenadas no sistema retangular global $x_{1} x_{2} x_{3}$ interpolando linearmente as coordenadas dos nós localizados nas extremidades do segmento integrado. Portanto:

$$
x_{i}=x_{i}^{0}+\left[\left(x_{i}^{f}-x_{i}^{0}\right) \frac{(\zeta+1)}{2}\right]
$$

O índice subscrito $i$ indica a direção da coordenada global determinada, enquanto os índices sobrescritos 0 e $f$ indicam, respectivamente, o ponto inicial e o final do segmento de reta. Para saber qual é o nó inicial e qual é o final basta verificar a orientação da coordenada $\zeta$, a qual segue o sentido do giro positivo em relação ao versor normal $\eta_{j}$ do elemento. Para determinar as coordenadas do ponto campo no sistema local $\bar{x}_{1} \bar{x}_{2} \bar{x}_{3}$ pode-se empregar a mesma expressão, ou seja:

$$
\bar{x}_{i}=\bar{x}_{i}^{0}+\left[\left(\bar{x}_{i}^{f}-\bar{x}_{i}^{0}\right) \frac{(\zeta+1)}{2}\right]
$$

A partir das coordenadas dos pontos fonte e campo nos dois sistemas retangulares é possível determinar, a partir de igualdades apresentadas anteriormente, todos os termos 
necessários para o cálculo das expressões 2.180 e 2.181.

\subsubsection{Sistema de equações}

A equação 2.116 é válida para um nó $i$ qualquer pertencente ao domínio $\Omega$ do sólido. Para simplificar a implementação desta equação, é mais interessante que os somatórios sejam em nós ao invés de elementos. Para efetuar esta transformação, são definidas as relações:

$$
\begin{aligned}
\hat{\mathbf{H}}_{i j} & =\sum_{t} \int_{\Gamma_{t}} \mathbf{p}^{*} \boldsymbol{\Phi}_{q} d \Gamma \\
\mathbf{G}_{i j} & =\sum_{t} \int_{\Gamma_{t}} \mathbf{u}^{*} \boldsymbol{\Phi}_{q} d \Gamma
\end{aligned}
$$

Nestas relações, o índice $i$ é o número do nó onde se encontra o ponto campo e o índice j é o número do nó onde se encontra o ponto fonte. O índice $q$ indica a numeração local do nó $j$ no elemento $t$. A soma referenciada por $t$ vai de 1 até o número de elementos dos quais o nó $j$ é vértice.

Assim, com as relações 2.184 e 2.185, pode-se reescrever a equação 2.116 como:

$$
\mathbf{c}^{i} \mathbf{u}^{i}+\sum_{j=1}^{3 n} \hat{\mathbf{H}}_{i j} \mathbf{u}^{j}=\sum_{j=1}^{3 n} \mathbf{G}_{i j} \mathbf{p}^{j}
$$

em que $n$ é o número de nós no contorno. Para simplificar a equação 2.186, impõem-se as condições:

$$
\begin{gathered}
\mathbf{H}_{i j}=\hat{\mathbf{H}}_{i j} \text { para } i \neq j \\
\mathbf{H}_{i j}=\hat{\mathbf{H}}_{i j}+\mathbf{c}^{i} \text { para } i=j
\end{gathered}
$$

Substituindo $\mathbf{H}_{\mathbf{i j}}$, dado nas relações 2.187 e 2.188, na equação 2.186, obtém-se:

$$
\sum_{j=1}^{3 n} \mathbf{H}_{i j} \mathbf{u}^{j}=\sum_{j=1}^{3 n} \mathbf{G}_{i j} \mathbf{p}^{j}
$$

A equação 2.189 pode ser escrita para todos os nós do contorno, resultando um sistema de $3 n$ equações cuja forma matricial é:

$$
\mathbf{H u}=\mathbf{G p}
$$

Conforme mostrado nas relações 2.187 e 2.188, os termos $\mathbf{c}^{i}$ são computados na matriz 
H. Eles compõem uma série de submatrizes de dimensão $3 \times 3$, somadas na região da diagonal principal de $\mathbf{H}$. Para encontrar os valores das submatrizes $\mathbf{c}^{i}$ em casos gerais nos quais o contorno $\Gamma$ não é suave, pode-se aplicar propriedades da matriz $\mathbf{H}$ decorrentes de movimentos de corpo rígido. Isto será visto mais adiante, na Seção 2.5.4.

Para que se possa resolver o problema do valor de contorno a partir das equações representadas por 2.190, é necessário adaptar este sistema de equações às condições de contorno do problema. Isto pode ser feito prescrevendo, para cada direção de cada ponto do contorno, um valor de deslocamento ou um valor de força. Desta forma, ambos os vetores $\mathbf{u}$ e $\mathbf{p}$ se tornam parcialmente conhecidos e parcialmente incógnitos. Além disto, caso o valor de deslocamento em uma determinada linha de u seja prescrito, o valor nesta mesma linha de $\mathbf{p}$ obrigatoriamente deve estar incógnito e vice-versa. Desta forma, o número de incógnitas se torna igual ao número de equações e o sistema tem solução única.

Para obter os valores de contorno incógnitos na equação 2.190, é necessário que todos eles estejam no mesmo lado da equação. Da mesma forma, todos os valores prescritos devem estar do outro lado. Isto pode ser feito a partir da equação 2.190, passando para o lado esquerdo cada valor incógnito de $\mathbf{p}$ e o substituindo no lado direito pelo correspondente conhecido de $\mathbf{u}$. As matrizes $\mathbf{H}$ e $\mathbf{G}$ também devem ser modificadas, trocando entre elas a coluna correspondente à linha de $\mathbf{p}$ e $\mathbf{u}$ para a qual foi feita a troca. Repetindo este procedimento para todas as forças incógnitas, obtém-se a equação:

$$
\mathbf{A x}=\mathbf{B y}
$$

$\mathrm{Na}$ equação 2.191, todos os termos do vetor $\mathbf{x}$ são incógnitos e todos os termos do vetor y são conhecidos. Além disto, todos os termos das matrizes A e B também são conhecidos, pois elas são resultado da troca de colunas entre as matrizes $\mathbf{H}$ e $\mathbf{G}$. Por isto, pode-se fazer o produto entre a matriz $\mathbf{B}$ e o vetor $\mathbf{y}$ à direita da igualdade. O resultado é um sistema de $3 n$ equações com $3 n$ incógnitas, ou seja:

$$
\mathrm{Ax}=\mathbf{f}
$$

Pela resolução do sistema 2.192 podem ser obtidos todos os valores de contorno incógnitos, resolvendo o problema elástico tridimensional do valor de contorno. 


\subsubsection{Movimentos de corpo rígido}

O conceito de movimento de corpo rígido envolve deslocamentos na ausência de forças. Isto implica em prescrever todas as forças no contorno $\Gamma$ do sólido iguais a zero. Ao fazer isto na equação 2.190, o produto Gp fica igual a zero. Assim:

$$
\mathbf{H u}=\mathbf{0}
$$

A interpretação física do problema de valor de contorno representado pela equação 2.193 é de um sólido sem restrição alguma, portanto solto no espaço. Extendendo esta interpretação, uma configuração possível é deslocar todos os nós do contorno do sólido de uma distância unitária no sentido positivo de um dos eixos coordenados. Escolhendo o eixo $x_{1}$, por exemplo, o vetor $\mathbf{u}_{1}$ para um movimento de corpo rígido unitário positivo em sua direção é:

$$
\left\{u_{i}\right\}^{T}=\left\{\begin{array}{llllllllll}
1 & 0 & 0 & 1 & 0 & 0 & \cdots & 1 & 0 & 0
\end{array}\right\}
$$

O produto de uma linha $i$ qualquer da matriz $\mathbf{H}$ pelo vetor $\mathbf{u}_{1}$ deve ser igual a zero, conforme imposto na equação 2.193. Assim, obtém-se uma importante propriedade da matriz $\mathbf{H}$, que é:

$$
H_{1}^{i}+H_{4}^{i}+H_{7}^{i}+\cdots+H_{(3 n-2)}^{i}=0
$$

O mesmo pode ser feito para as direções de $x_{2}$ e $x_{3}$, obtendo outras duas equações:

$$
\begin{gathered}
H_{2}^{i}+H_{5}^{i}+H_{8}^{i}+\cdots+H_{(3 n-1)}^{i}=0 \\
H_{3}^{i}+H_{6}^{i}+H_{9}^{i}+\cdots+H_{(3 n)}^{i}=0
\end{gathered}
$$

As equações 2.195, 2.196 e 2.197 podem ser utilizadas para determinar indiretamente o valor dos termos das submatrizes $\mathbf{c}^{i}$. Depois da obtenção da matriz $\hat{\mathbf{H}}$, percorre-se cada linha $i$ com as operações:

$$
\begin{aligned}
R_{1}^{i} & =\sum_{j=1}^{n}\left[\hat{H}_{(3 j-2)}^{i}\right] \\
R_{2}^{i} & =\sum_{j=1}^{n}\left[\hat{H}_{(3 j-1)}^{i}\right] \\
R_{3}^{i} & =\sum_{j=1}^{n}\left[\hat{H}_{(3 j)}^{i}\right]
\end{aligned}
$$


em que $R_{1}^{i}, R_{2}^{i}$ e $R_{3}^{i}$ contêm a soma dos termos de cada linha $i$ da matriz $\hat{\mathbf{H}}$, correspondentes aos movimentos de corpo rígido nas direções $x_{1}, x_{2}$ e $x_{3}$, respectivamente. Para obter a matriz final $\mathbf{H}$, utilizada na equação 2.190 , basta subtrair os termos $R_{1}^{i}, R_{2}^{i}$ e $R_{3}^{i}$ calculados para cada linha das posições correspondentes de cada linha. Assim, os três termos corretores da submatriz $\mathbf{c}^{i}$ para uma linha $i$ qualquer de $\hat{\mathbf{H}}$ são:

$$
\begin{aligned}
& c_{1}^{i}=-R_{1}^{i} \\
& c_{2}^{i}=-R_{2}^{i} \\
& c_{3}^{i}=-R_{3}^{i}
\end{aligned}
$$

A posição na linha $i$ da matriz $\hat{\mathbf{H}}$ na qual cada termo de $\mathbf{c}^{i}$ deve ser somado pode ser encontrada a partir do número $j$ do nó ao qual pertence o deslocamento em questão. Aplicando a regra imposta pela relação 2.188, conclui-se que o termo $c_{1}^{i}$ deve ser somado na posição $3 j-2$ da linha, o termo $c_{2}^{i}$ deve ser somado na posição $3 j-1$ e o termo $c_{3}^{i}$ deve ser somado na posição $3 j$.

Apesar de estar resolvido o problema da submatriz $\mathbf{c}^{i}$, deve-se ressaltar que os deslocamentos segundo os três eixos coordenados não são os únicos movimentos de corpo rígido possíveis. Ainda podem ocorrer três rotações, uma para cada eixo. Assim, caso se tenha dúvida quanto à matriz $\mathbf{H}$ mesmo após a correção com as submatrizes $\mathbf{c}^{i}$, ainda é possível conferir suas propriedades quanto aos movimentos de corpo rígido rotacionais.

\subsubsection{Pontos internos}

Após determinar todos os valores de contorno é possível, a partir deles, determinar deslocamentos e tensões em qualquer ponto interno ao domínio $\Omega$ do sólido. Para obter uma equação cuja solução sejam os deslocamentos em pontos internos, primeiramente deve-se voltar à equação 2.76 com $b_{k}$ igual a zero, ou seja:

$$
u_{l}^{i}+\int_{\Gamma} u_{k} p_{l k}^{*} d \Gamma=\int_{\Gamma} p_{k} u_{l k}^{*} d \Gamma
$$

Para resolver a equação 2.204 para um ponto no contorno $\Gamma$ do sólido, foi necessário analisar a integral à direta da igualdade em 2.204 de forma especial, devido à singularidade 
no ponto. Porém, quando se considera um ponto interno ao domínio $\Omega$ do sólido, não há sigularidade porque o ponto fonte nunca coincide com o ponto campo. Assim, pode-se efetuar ambas as integrais da equação 2.204 sem se preocupar com singularidades. A partir da dedução feita para o ponto do contorno, aplicando os mesmos procedimentos numéricos entre as equações 2.76 e 2.186 na equação 2.204 , pode-se deduzir que a equação resultante é a seguinte:

$$
\mathbf{u}^{i}=\sum_{j=1}^{3 n} \mathbf{G}_{i j} \mathbf{p}^{j}-\sum_{j=1}^{3 n} \mathbf{H}_{i j} \mathbf{u}^{j}
$$

Na equação 2.205, $n$ corresponde ao número de nós, $i$ correponde ao número do nó interno ou ponto fonte e $j$ é o número do nó do contorno ou ponto campo. A partir dela, pode-se determinar diretamente o deslocamento em qualquer ponto interno.

Além dos deslocamentos, também é possível determinar o tensor de tensões em um ponto interno $i$. Para isto, primeiramente é necessário encontrar a equação diferencial em deslocamentos que permite obter tensões no interior de um sólido elástico. Parte-se da relação 2.23, que é:

$$
\varepsilon_{i j}=\frac{u_{i, j}+u_{j, i}}{2}
$$

Outra expressão a ser utilizada é a seguinte relação constitutiva:

$$
\sigma_{i j}=\frac{E}{(1+\nu)}\left[\frac{\nu}{(1-2 \nu)} \delta_{i j} \varepsilon_{k k}+\varepsilon_{i j}\right]
$$

Substituindo a relação 2.206 na expressão 2.207, obtém-se a expressão:

$$
\sigma_{i j}=\frac{E}{(1+\nu)}\left[\frac{\nu}{(1-2 \nu)} \delta_{i j} u_{k, k}+\frac{1}{2}\left(u_{i, j}+u_{j, i}\right)\right]
$$

$\mathrm{Ou}$

$$
\sigma_{i j}=\frac{2 \nu G}{(1-2 \nu)} \delta_{i j} u_{l, l}+\left(u_{i, j}+u_{j, i}\right) G
$$

Isolando o deslocameto $u_{l}^{i}$ na equação 2.204 e substituindo em 2.209, obtém-se:

$$
\begin{aligned}
\sigma_{i j} & =\int_{\Gamma}\left\{\frac{2 \nu G}{(1-2 \nu)} \delta_{i j} u_{l k, l}^{*}+\left(u_{i k, j}^{*}+u_{j k, i}^{*}\right) G\right\} p_{k} d \Gamma+ \\
& -\int_{\Gamma}\left\{\frac{2 \nu G}{(1-2 \nu)} \delta_{i j} p_{l k, l}^{*}+\left(p_{i k, j}^{*}+p_{j k, i}^{*}\right) G\right\} u_{k} d \Gamma
\end{aligned}
$$

Todas as derivadas da expressão 2.210 são tomadas no ponto interno, considerando onde está sendo aplicada a solução fundamental. Esta relação pode ser reescrita de forma 
mais compacta da seguinte maneira:

$$
\sigma_{i j}=\int_{\Gamma} D_{k i j} p_{k} d \Gamma-\int_{\Gamma} S_{k i j} u_{k} d \Gamma
$$

Em que os termos $D_{k i j}$ e $S_{k i j}$ são tensores. Estes tensores são dados por:

$$
\begin{gathered}
D_{k i j}=\frac{1}{8 \pi(1-\nu) r^{2}}\left\{(1-2 \nu)\left[\delta_{k i} r_{, j}+\delta_{k j} r_{, i}-\delta_{i j} r_{, k}\right]+3 r_{, k} r_{, i} r_{, j}\right\} \\
S_{k i j}=\frac{G}{4 \pi(1-\nu) r^{3}}\left\{3 r_{, \eta}\left[(1-2 \nu) \delta_{i j} r_{, k}+\nu\left(\delta_{i k} r_{, j}+\delta_{j k} r_{, i}\right)-5 r_{, k} r_{, i} r_{, j}\right]+\right. \\
\left.+3 \nu\left(\eta_{i} r_{, j} r_{, k}+\eta_{j} r_{, i} r_{, k}\right)+(1-2 \nu)\left(3 \eta_{k} r_{, i} r_{, j}+\eta_{j} \delta_{i k}+\eta_{i} \delta_{j k}\right)-(1-4 \nu) \eta_{k} \delta_{i j}\right\}
\end{gathered}
$$

\subsubsection{Subelementação}

Para melhorar a precisão dos resultados caso o ponto fonte esteja muito próximo ao elemento de contorno integrado (EC), divide-se este elemento em áreas menores denominadas subelementos (SE). Desta forma, para cada SE, pode-se definir mais pontos de integração. Somando a influência de todos os SE, tem-se a contribuição total do EC integrado.

Em Almeida (2003b) dois tipos de subelementação são empregadas, a convencional e a progressiva. Na subelementação convencional o EC é dividido de forma regular, conforme mostrado na figura 2.13 .

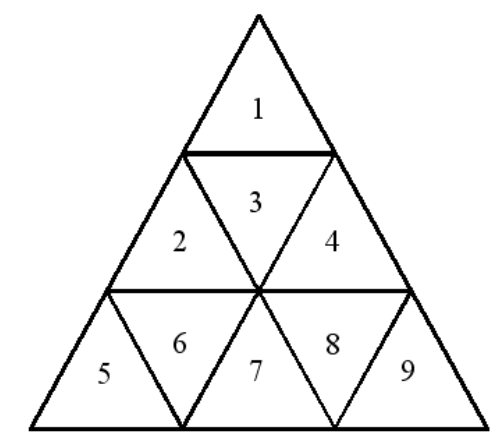

Figura 2.13: Subelementação convencional

O tamanho dos SE é definido a partir da distância do ponto fonte ao EC em questão. Caso o ponto fonte esteja próximo são definidos SE pequenos e em grande quantidade, e caso esteja muito distante basta um único SE para que a integral seja precisa.

Na subelementação progressiva a divisão do EC é mais intensa na região mais próxima ao ponto fonte. Na figura 2.14, por exemplo, é mostrada uma possível configuração quando 
o ponto fonte se encontra próximo ao vértice $P$ do EC.

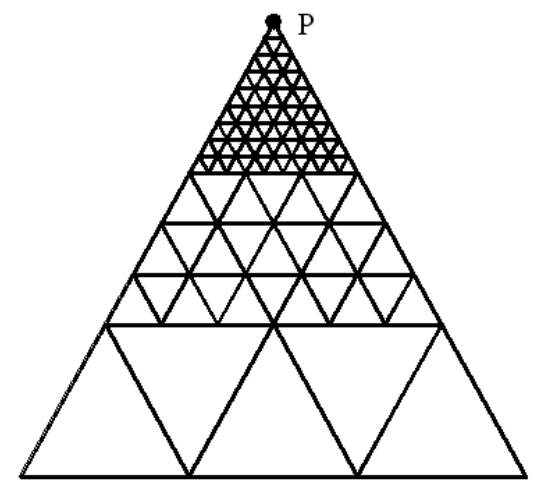

Figura 2.14: Subelementação progressiva

Após alguns testes no programa concluiu-se que, como a integral singular é calculada semi-analiticamente, a subelementação progressiva é desnecessária. Caso exista uma situação quase singular, em que o ponto fonte esteja próximo ao EC a ser integrado, a subelementação regular é suficiente para garantir uma boa precisão. Desta forma, neste trabalho é empregada somente subelementação regular. Na figura 2.15 é ilustrado um SE genérico posicionado dentro do EC a ser integrado.

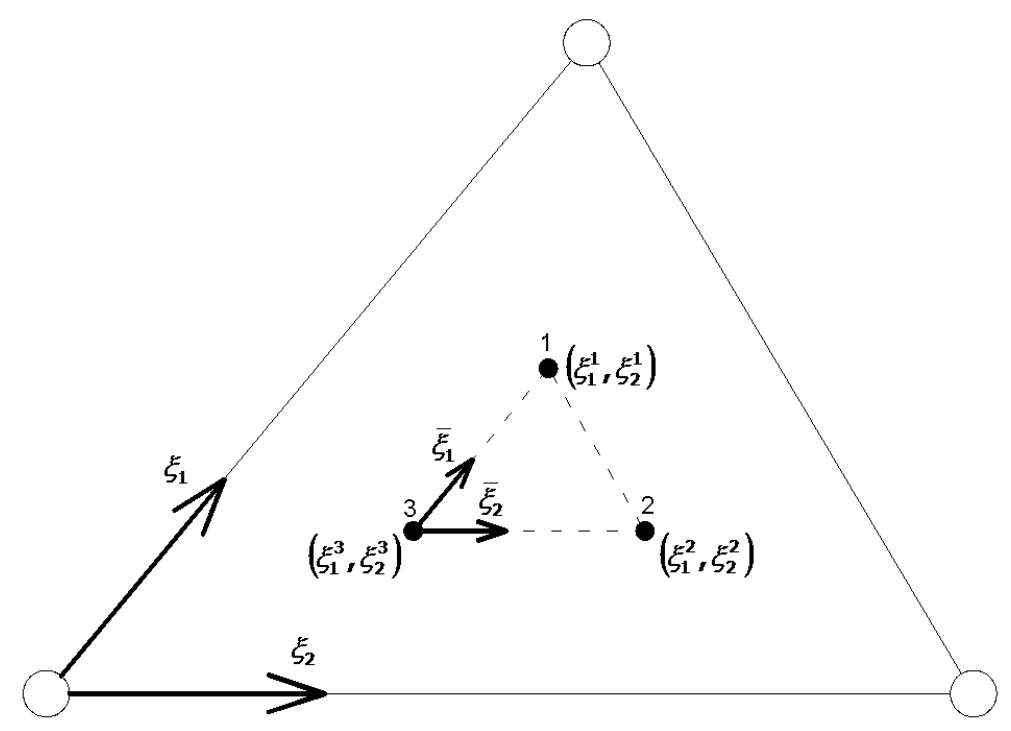

Figura 2.15: Subelemento genérico

Além do sistema homogêneo local do EC, $\xi_{1} \xi_{2}$, um outro sistema homogêneo, $\bar{\xi}_{1} \bar{\xi}_{2}$, é introduzido para cada SE. Os vértices do SE são numerados localmente e as coordenadas destes vértices no sistema $\xi_{1} \xi_{2}$ estão indicadas na figura, sendo que o índice subscrito indica a direção e o sobrescrito indica a numeração local do vértice. A utilização de SEs 
implica em uma alteração na expressão 2.116, que se torna:

$$
\mathbf{c}^{i} \mathbf{u}^{i}+\sum_{j=1}^{n e}\left\{|\mathbf{J}|^{j}\left[\sum_{s=1}^{n s}|\mathbf{J}|^{s}\left(\sum_{k=1}^{n h}\left\{\mathbf{w}_{k} \mathbf{p}_{k}^{*} \boldsymbol{\Phi}_{k}\right\}\right)\right] \mathbf{u}^{j}\right\}=\sum_{j=1}^{n e}\left\{|\mathbf{J}|^{j}\left[\sum_{s=1}^{n s}|\mathbf{J}|^{s}\left(\sum_{k=1}^{n h}\left\{\mathbf{w}_{k} \mathbf{u}_{k}^{*} \boldsymbol{\Phi}_{k}\right\}\right)\right] \mathbf{p}^{j}\right\}
$$

Como cada EC está dividido em um certo número de SEs, $n_{s}$, mais um somatório foi incluído na expressão. É importante observar que surge mais um Jacobiano a ser considerado, o qual é identificado como $|\mathbf{J}|^{s}$. Para determinar este Jacobiano é necessário, inicialmente, relacionar os sistemas $\xi_{1} \xi_{2}$ e $\bar{\xi}_{1} \bar{\xi}_{2}$. Neste trabalho, estes sistemas se relacionam da seguinte forma:

$$
\begin{aligned}
& \xi_{1}=\xi_{1}^{1} \bar{\xi}_{1}+\xi_{1}^{2} \bar{\xi}_{2}+\xi_{1}^{3} \bar{\xi}_{3} \\
& \xi_{2}=\xi_{2}^{1} \bar{\xi}_{1}+\xi_{2}^{2} \bar{\xi}_{2}+\xi_{2}^{3} \bar{\xi}_{3}
\end{aligned}
$$

É importante salientar que a coordenada $\bar{\xi}_{3}$ não é independente das outras duas, pois $\bar{\xi}_{3}=1-\bar{\xi}_{1}-\bar{\xi}_{2}$. Desta forma, calcula-se as derivadas das expressões 2.215 e 2.216 como segue:

$$
\begin{array}{ll}
\frac{\partial \xi_{1}}{\partial \bar{\xi}_{1}}=\xi_{1}^{1}-\xi_{1}^{3}, & \frac{\partial \xi_{1}}{\partial \bar{\xi}_{2}}=\xi_{1}^{2}-\xi_{1}^{3} \\
\frac{\partial \xi_{2}}{\partial \bar{\xi}_{1}}=\xi_{2}^{1}-\xi_{2}^{3}, & \frac{\partial \xi_{2}}{\partial \bar{\xi}_{2}}=\xi_{2}^{2}-\xi_{2}^{3}
\end{array}
$$

Obtidas as derivadas torna-se possível o cálculo do Jacobiano. Assim:

$$
|\mathbf{J}|^{s}=\left|\begin{array}{cc}
\frac{\partial \xi_{1}}{\partial \xi_{1}} & \frac{\partial \xi_{2}}{\partial \xi_{1}} \\
\frac{\partial \xi_{1}}{\partial \xi_{2}} & \frac{\partial \xi_{2}}{\partial \xi_{2}}
\end{array}\right|=\frac{\partial \xi_{1}}{\partial \bar{\xi}_{1}} \frac{\partial \xi_{2}}{\partial \bar{\xi}_{2}}-\frac{\partial \xi_{2}}{\partial \bar{\xi}_{1}} \frac{\partial \xi_{1}}{\partial \bar{\xi}_{2}}=\left(\xi_{1}^{1}-\xi_{1}^{3}\right)\left(\xi_{2}^{2}-\xi_{2}^{3}\right)-\left(\xi_{2}^{1}-\xi_{2}^{3}\right)\left(\xi_{1}^{2}-\xi_{1}^{3}\right)
$$

\subsubsection{Linhas de carga aplicadas ao meio tridimensional}

Neste trabalho são consideradas estruturas de barra ligadas ao meio tridimensional. Estas estruturas representam estacas e são modeladas pelo método dos elementos finitos (MEF), conforme será explicado com mais detalhes no Capítulo 5. Para que esta ligação seja possível é necessário considerar, na formulação do MEC, as forças de interação decorrentes da presença de tais estruturas. Isto pode ser feito de diferentes formas, e a estratégia escolhida neste trabalho consiste em considerar as forças de interação como linhas de carga aplicadas do meio tridimensional. Esta estratégia tem a vantagem de ser de sim- 
ples implementação, além de fornecer resultados precisos para as aplicações pretendidas neste trabalho. A redação desta Seção foi auxiliada pelos trabalhos de Venturini (1988), Filho (1999) e Filho et al. (2005), que empregam a mesma formulação e cuja consulta é aqui recomendada.

Considera-se uma linha de carga $\Gamma^{e}$ qualquer aplicada a um sólido tridimensional de domínio $\Omega$ e contorno $\Gamma$, conforme ilustrado na figura 2.16. A linha de carga possui componentes nas três direções do sistema de coordenadas global e sua presença implica no surgimento de mais um termo na identidade de Somigliana, representada pela expressão 2.83, que se torna:

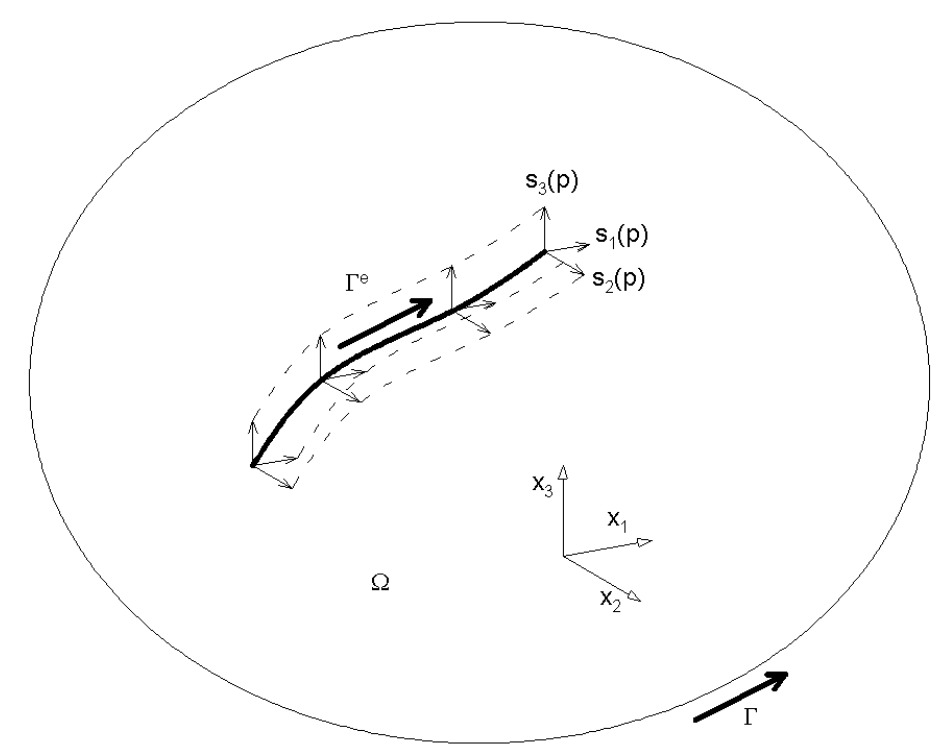

Figura 2.16: Linha de carga genérica

$$
c_{l k}^{i}+\int_{\Gamma} p_{l k}^{*} u_{k} d \Gamma=\int_{\Gamma} u_{l k}^{*} p_{k} d \Gamma+\int_{\Omega} u_{l k}^{*} b_{k} d \Omega+\int_{\Gamma^{e}} u_{l k}^{*} s_{k}^{e} d \Gamma
$$

O termo adicional corresponde à integral tomada ao longo da linha de carga $\Gamma^{e}$, em que $u_{l k}^{*}$ é a solução fundamental de deslocamento, conforme descrito anteriormente, e $s_{k}^{e}$ é o valor da linha de carga $e$ na direção $k$. Igualando as cargas de domínio $b_{k}$ a zero e generalizando a expressão para um número qualquer de linhas de carga, obtém-se:

$$
c_{l k}^{i}+\int_{\Gamma} p_{l k}^{*} u_{k} d \Gamma=\int_{\Gamma} u_{l k}^{*} p_{k} d \Gamma+\sum_{e=1}^{n t o t} \int_{\Gamma^{e}} u_{l k}^{*} s_{k}^{e} d \Gamma
$$

em que ntot é o número total de linhas de carga. 
O passo seguinte é reescrever a expressão 2.221 considerando que o contorno $\Gamma$ do sólido está dividido em elementos, ao longo dos quais os deslocamentos e forças são aproximados por funções conhecidas. O mesmo é feito para as linhas de carga, ou seja, adota-se funções conhecidas para aproximá-las. O resultado é o aparecimento de mais um termo na expressão 2.103 que se torna:

$$
\mathbf{c}^{i} \mathbf{u}^{i}+\sum_{j=1}^{n e}\left[\left(\int_{\Gamma^{j}} \mathbf{p}^{*} \boldsymbol{\Phi} d \Gamma\right) \mathbf{u}^{j}\right]=\sum_{j=1}^{n e}\left[\left(\int_{\Gamma^{j}} \mathbf{u}^{*} \mathbf{\Phi} d \Gamma\right) \mathbf{p}^{j}\right]+\sum_{e=1}^{n t o t}\left[\left(\int_{\Gamma^{e}} \mathbf{u}^{*} \overline{\boldsymbol{\Phi}} d \Gamma\right) \mathbf{s}^{e}\right]
$$

No termo adicional decorrente da presença das linhas de carga, $\overline{\mathbf{\Phi}}$ representa as funções de forma adotadas para aproximar as cargas e $\mathbf{s}^{e}$ é um vetor que contém o valor das cargas nos nós definidos ao longo de cada linha. Torna-se então necessário adotar características mais específicas para as linhas de carga, e essas características são apresentadas na figura 2.17 .

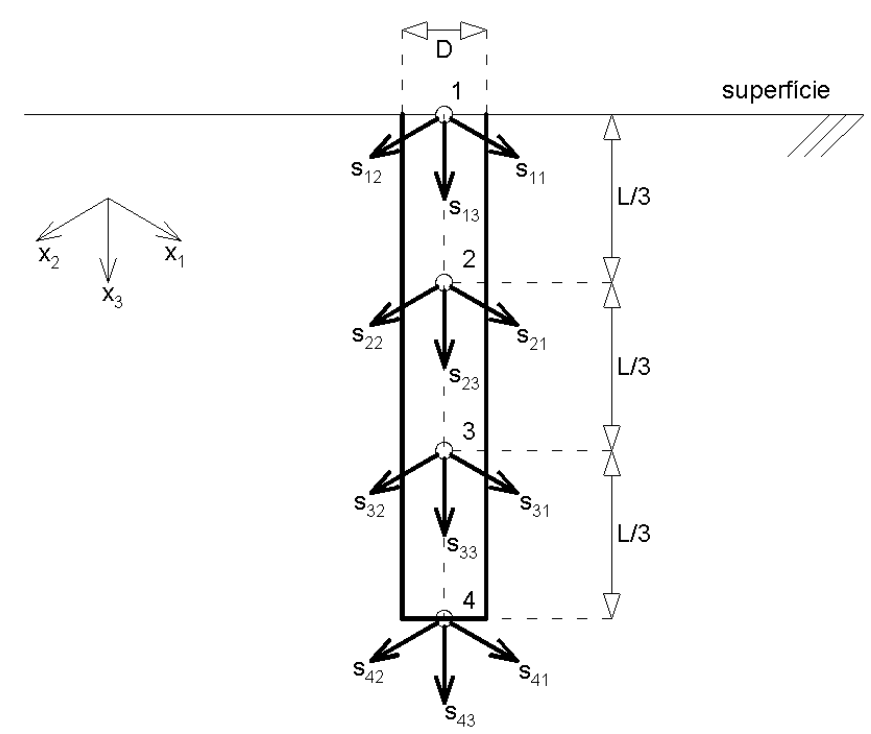

Figura 2.17: Características das linhas de carga

A figura apresenta uma das linhas de carga e a estaca que esta linha representa. As linhas de carga são consideradas retilíneas e verticais, ou seja, alinhadas com a coordenada $x_{3}$ do sistema de coordenadas global. Cada uma possui quatro nós distribuídos de forma regular ao longo do comprimento $L$, e são definidos três graus de liberdade por nó. Cada um destes doze graus de liberdade está representado na figura como $s_{i j}$, sendo $i$ o número do nó e $j$ a direção no sistema de coordenadas global.

Apesar dos nós estarem posicionados no eixo da estaca, considera-se que esta possui 
um diâmetro $D$ diferente de zero. Desta forma, quando a equação 2.222 é escrita para um dos nós da estaca o deslocamento é calculado no nó mas as forças são aplicadas no fuste, evitando assim o surgimento de singularidades nas integrais. Por outro lado, quando o ponto fonte não pertence à linha de carga a ser integrada, esta estratégia se torna desnecessária e portanto as cargas são aplicadas no eixo central da estaca.

Observando a figura 2.17, conclue-se que o único caso em que a singularidade ocorre é quando o ponto fonte é posicionado no nó de base de uma das estacas e esta base é integrada. Considera-se que a superfície da base transmite carga somente no sentido do eixo da estaca, ou seja, na direção $x_{3}$ do sistema global. Portanto, a contribuição do nó da extremidade nas direções $x_{1}$ e $x_{2}$ é totalmente transmitida para o fuste, evitando assim a singularidade nestas direções. Por outro lado, a contribuição deste mesmo nó na direção $x_{3}$ é totalmente transmitida para a base, isolando o caso singular. Tendo em vista estes fatores, parte-se para a obtenção das funções de forma.

Inicia-se com as componentes horizontais, ou seja, nas direções $x_{1}$ e $x_{2}$. Estas componentes são transmitidas pelo fuste da estaca, que recebe a influência dos quatro nós. Portanto, os carregamentos $q$ na direção $x_{1}$ e $p$ na direção $x_{2}$ podem ser escritos como:

$$
\begin{aligned}
& q=\{\phi\}^{T}\left\{q_{i}\right\}=\left\{\begin{array}{llll}
\phi_{1} & \phi_{2} & \phi_{3} & \phi_{4}
\end{array}\right\}\left\{\begin{array}{l}
q_{1} \\
q_{2} \\
q_{3} \\
q_{4}
\end{array}\right\} \\
& p=\{\phi\}^{T}\left\{p_{i}\right\}=\left\{\begin{array}{llll}
\phi_{1} & \phi_{2} & \phi_{3} & \phi_{4}
\end{array}\right\}\left\{\begin{array}{c}
p_{1} \\
p_{2} \\
p_{3} \\
p_{4}
\end{array}\right\}
\end{aligned}
$$

Como se pretende utilizar funções polinomiais, o ideal para quatro pontos é empregar polinômios de terceiro grau. Utiliza-se uma variável adimensional $\xi$ tal que:

$$
\xi=\frac{2 x_{3}}{L}-1
$$


Assim, chega-se às seguintes funções:

$$
\begin{gathered}
\phi_{1}=\frac{1}{16}\left(-9 \xi^{3}+9 \xi^{2}+\xi-1\right) \\
\phi_{2}=\frac{1}{16}\left(27 \xi^{3}-9 \xi^{2}-27 \xi+9\right) \\
\phi_{3}=\frac{1}{16}\left(-27 \xi^{3}-9 \xi^{2}+27 \xi+9\right) \\
\phi_{4}=\frac{1}{16}\left(9 \xi^{3}+9 \xi^{2}-\xi-1\right)
\end{gathered}
$$

O passo seguinte é analisar as componentes cisalhantes verticais no fuste, ou seja, na direção $x_{3}$. Somente três nós contribuem no equilíbrio vertical do fuste, pois o nó da base contribui somente na área da base. Assim, o carregamento no fuste $\tau$ na direção $x_{3}$ pode ser escrito como:

$$
\tau=\{\omega\}^{T}\left\{\tau_{i}\right\}=\left\{\begin{array}{lll}
\omega_{1} & \omega_{2} & \omega_{3}
\end{array}\right\}\left\{\begin{array}{c}
\tau_{1} \\
\tau_{2} \\
\tau_{3}
\end{array}\right\}
$$

Como são três pontos, os polinômios ideais neste caso são os de segundo grau. Empregando novamente a variável adimensional $\xi$, obtém-se:

$$
\begin{gathered}
\omega_{1}=\frac{1}{8}\left(9 \xi^{2}-1\right) \\
\omega_{2}=\frac{1}{4}\left(-9 \xi^{2}-6 \xi+3\right) \\
\omega_{3}=\frac{1}{8}\left(9 \xi^{2}+12 \xi+3\right)
\end{gathered}
$$

Resta definir a função de forma que aproxima a força axial transmitida pela base, lembrando que é neste caso que ocorre a singularidade. Como o polinômio é baseado em um único valor nodal assume-se que a carga é constante, ou seja, uniformemente distribuída na base. O fato desta função ser constante é vantajoso, pois isto simplifica o tratamento especial a ser dado à singularidade.

Por fim, são definidas formas eficientes e precisas de calcular as integrais da expressão 2.222 que envolvem linhas de carga. Inicialmente, separa-se de 2.222 o termo a ser estu- 
dado:

$$
\mathbf{I}=\sum_{e=1}^{n t o t}\left[\left(\int_{\Gamma^{e}} \mathbf{u}^{*} \overline{\boldsymbol{\Phi}} d \Gamma\right) \mathbf{s}^{e}\right]
$$

Na expressão 2.234, a integral calculada nas superfícies dos fustes das estacas não é tratada da mesma forma que a integral calculada nas superfícies das bases das estacas. Por este motivo, torna-se necessário separar as parcelas de fuste das parcelas de base. O resultado é:

$$
\mathbf{I}=\sum_{e=1}^{n t o t}\left[\left(\int_{\Gamma_{f}^{e}} \mathbf{u}^{*} \overline{\boldsymbol{\Phi}} d \Gamma\right) \mathbf{s}_{f}^{e}+\left(\int_{\Gamma_{b}^{e}} \mathbf{u}^{*} \overline{\mathbf{\Phi}} d \Gamma\right) \mathbf{s}_{b}^{e}\right]
$$

Em 2.235, o termo $\Gamma_{f}^{e}$ representa a superfície do fuste da estaca e o termo $\Gamma_{b}^{e}$ representa a superfície da base da estaca. O vetor $\mathbf{s}_{b}^{e}$ contém a tensão vertical de contato na base, e o vetor $\mathbf{s}_{f}^{e}$ contém as tensões no fuste. Como as integrais do fuste são tratadas de forma diferente das integrais da base, analisa-se inicialmente de forma separada a parcela referente ao fuste de uma estaca. Assim:

$$
\mathbf{I}_{f}^{e}=\int_{\Gamma_{f}^{e}} \mathbf{u}^{*} \overline{\boldsymbol{\Phi}} d \Gamma
$$

Neste trabalho, a integral 2.236 é sempre calculada numericamente empregando pontos de Gauss. Para que isto seja possível, é necessário primeiramente passar do sistema de coordenadas global para o local de cada estaca. Sendo esta transformação equivalente à que foi feita na Seção 2.5.2, figura 2.12, conclue-se que o Jacobiano é igual a $L_{e} / 2$, sendo $L_{e}$ o comprimento da estaca.

Além disso deve-se atentar ao fato de que, apesar da estaca ser representada por uma linha de carga, considera-se que tal estaca possua um diâmetro $D_{e}$ diferente de zero. Desta forma, além do Jacobiano referente à transformação do sistema global para o local, deve-se multiplicar as contribuições do fuste pelo perímetro da estaca. Desta forma, após estas considerações, conclue-se que a expressão 2.236 transformada para o sistema local se torna:

$$
\mathbf{I}_{f}^{e}=\frac{\pi D_{e} L_{e}}{2} \int_{-1}^{+1} \mathbf{u}^{*} \overline{\boldsymbol{\Phi}} d \xi
$$

O passo seguinte é representar a integral 2.237 por um somatório de contribuições dos 
pontos de Gauss, obtendo:

$$
\mathbf{I}_{f}^{e}=\frac{\pi D_{e} L_{e}}{2}\left[\sum_{k=1}^{n g}\left(\mathbf{w}_{k} \mathbf{u}_{k}^{*} \overline{\mathbf{\Phi}}_{k}\right)\right]
$$

em que $n g$ é o número de pontos de Gauss e $\mathbf{w}_{k}$ é o peso de cada ponto de Gauss. A expressão 2.238 é adequada para calcular a integral no fuste quando o ponto fonte está distante, mas pode perder precisão nos casos quase-singulares. Em vista disto, a solução adotada neste trabalho para melhorar a precisão do cálculo da integral quase-singular é subdividir a linha de carga em subelementos, de forma semelhante à empregada para os elementos de contorno triangulares. Este procedimento torna necessário transformar novamente o sistema de coordenadas, gerando mais um Jacobiano na expressão. O cálculo deste Jacobiano adicional é análogo ao da transformação do sistema global para o local, ou seja:

$$
\left|\mathbf{J}_{s}\right|=L_{s} / 2
$$

em que

$$
L_{s}=\left|\xi_{a}-\xi_{b}\right|
$$

sendo $\xi_{a}$ e $\xi_{b}$ as coordenadas adimensionais das extremidades do subelemento. Por fim, reescreve-se a expressão 2.238 como:

$$
\mathbf{I}_{f}^{e}=\frac{\pi D_{e} L_{e}}{2}\left\{\sum_{s=1}^{n s}\left|\mathbf{J}_{s}\right|\left[\sum_{k=1}^{n g}\left(\mathbf{w}_{k} \mathbf{u}_{k}^{*} \overline{\mathbf{\Phi}}_{k}\right)\right]\right\}
$$

em que $n s$ é o número total de subelementos. Para não prejudicar a simetria dos resultados, o cálculo da expressão 2.241 é feito de forma especial quando o ponto fonte pertence à linha de carga integrada. Esta forma especial consiste em calcular a solução fundamental em pontos do fuste simétricos em relação ao eixo da linha de carga, conforme apresentado na figura 2.18 .

No lado esquerdo da figura 2.18 é apresentada a vista longitudinal da linha de carga com um dos pontos de integração. O ponto de integração está indicado como PI e suas projeções estão indicadas como $P^{\prime}$. Do lado direito é ilustrada a seção transversal que contém o ponto de integração, com suas quatro projeções e a solução fundamental de deslocamento em cada uma delas. Desta forma, para manter a simetria do problema, quando o ponto fonte está contido na linha de carga são calculadas quatro soluções fun- 


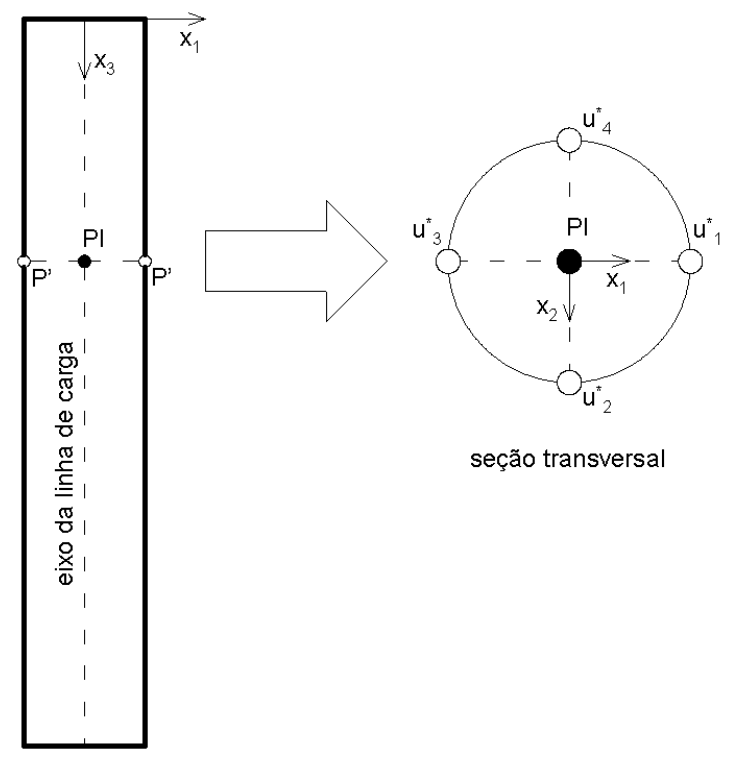

Figura 2.18: Projeções do ponto fonte

damentais por ponto de integração. A contribuição do ponto campo na direção $x_{1}$ do sistema global é calculada utilizando a média das soluções $u_{1}^{*}$ e $u_{3}^{*}$ indicadas na figura, a contribuição na direção $x_{2}$ utiliza a média entre $u_{2}^{*}$ e $u_{4}^{*}$ e, finalmente, a contribuição em $x_{3}$ emprega a média das quatro soluções.

Seguindo com a dedução, analisa-se separadamente a parcela de 2.235 referente à base de uma estaca. Deste modo:

$$
\mathbf{I}_{b}^{e}=\int_{\Gamma_{b}^{e}} \mathbf{u}^{*} \overline{\boldsymbol{\Phi}} d \Gamma
$$

Dois casos devem ser considerados no cálculo da integral 2.242, O primeiro é quando o ponto fonte não pertence à base integrada e portanto a integral não é singular, e o segundo é quando o ponto fonte está contido na base integrada, tornando-a singular.

Neste trabalho, no caso não-singular, a reação do solo na base da estaca é considerada como uma força concentrada e constante. Consequentemente, a solução fundamental é tomada em um único ponto, tornando-se constante em relação à área integrada. Isto permite retirar da integral a solução fundamental. Além disto a função de forma é unitária, podendo ser desconsiderada no cálculo. Por fim, obtém-se:

$$
\mathbf{I}_{b}^{e}=\mathbf{u}^{*} \int_{\Gamma_{b}^{e}} d \Gamma
$$

A integral é, neste caso, simplesmente igual à área da base da estaca. Portanto, sendo 
$A_{b}$ a área da base, o valor da integral é:

$$
\mathbf{I}_{b}^{e}=\mathbf{u}^{*} A_{b}
$$

No caso singular é necessária uma abordagem especial para que a precisão seja mantida, e neste trabalho optou-se pelo cálculo analítico da integral singular. Conforme justificado anteriormente, a situação em que singularidade ocorre é bem específica. A integral é singular caso a superfície integrada seja a base de uma estaca, o ponto fonte esteja posicionado no nó de base desta mesma estaca e a equação seja escrita para a direção $x_{3}$. Desta forma, conclui-se que a integral a ser calculada analiticamente é:

$$
I_{s}=\int_{A_{b}} u_{33}^{*} \sigma_{b} d A
$$

em que $A_{b}$ é a área da base da estaca, $\sigma_{b}$ é uma constante que representa a tensão de contato na base e

$$
u_{33}^{*}=\frac{1}{16 \pi \mu(1-\nu) r}\left[(3-4 \nu) \delta_{33}+r_{, 3} r_{, 3}\right]
$$

Como neste trabalho são consideradas apenas estacas verticais, a área a ser integrada no caso singular é sempre perpendicular ao eixo $x_{3}$ do sistema de coordenadas globais. Isto significa que a projeção de $r$ no eixo $x_{3}$ é sempre igual a zero no caso estudado, permitindo que a derivada $r_{, 3}$ seja igualada a zero. Além disso, a função Delta de Kronecker tem valor unitário quando seus dois índices são iguais. Substituindo estes valores em 2.246, obtémse:

$$
u_{33}^{*}=\frac{3-4 \nu}{16 \pi \mu(1-\nu) r}
$$

O primeiro passo é efetuar uma transformação do sistema global retangular para um sistema de coordenadas polares, conforme ilustrado na figura 2.19.

Foi demonstrado na Seção 2.5.2 que o Jacobiano da transformação de um sistema retangular para um de coordenadas polares é igual a $r$. Isto permite escrever:

$$
d A=d x d y=r d r d \theta
$$

Desta forma, utilizando o Jacobiano e substituindo a expressão de $u_{33}^{*}$, a nova integral 


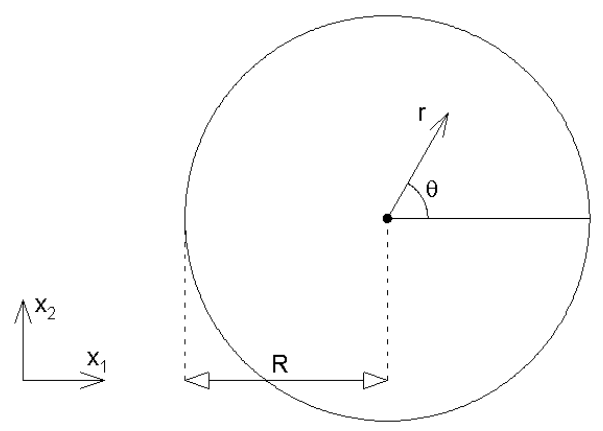

Figura 2.19: Transformação de coordenadas na base da estaca

a ser calculada é:

$$
I_{s}=\int_{0}^{2 \pi} \int_{0}^{R} \frac{3-4 \nu}{16 \pi \mu(1-\nu) r} \sigma_{b} r d r d \theta
$$

Observa-se que é possível cancelar a variável $r$ do numerador e denominador, obtendo:

$$
I_{s}=\int_{0}^{2 \pi} \int_{0}^{R} \frac{3-4 \nu}{16 \pi \mu(1-\nu)} \sigma_{b} d r d \theta
$$

A expressão integrada é constante em relação a $r$ e $\theta$, portanto pode ser retirada da integral. A expressão final é então obtida como segue:

$$
I_{s}=\frac{3-4 \nu}{16 \pi \mu(1-\nu)} \sigma_{b} \int_{0}^{2 \pi} \int_{0}^{R} d r d \theta=\frac{3-4 \nu}{16 \pi \mu(1-\nu)} \sigma_{b} 2 \pi R=\frac{\sigma_{b} R(3-4 \nu)}{8 \mu(1-\nu)}
$$

Assim, após definir formas eficientes de calcular todas as integrais, é possível retornar à expressão 2.222. Escrevendo-a para todos os pontos do contorno, obtém-se:

$$
\left[H_{c c}\right]\left\{u_{c}\right\}=\left[G_{c c}\right]\left\{p_{c}\right\}+\left[M_{c i}\right]\left\{s_{i}\right\}
$$

Em 2.252, o índice subscrito $c$ indica termos referentes a pontos do contorno e o índice $i$ indica termos referentes a pontos internos. Os coeficientes da matriz $\left[M_{c i}\right]$ são obtidos a partir da integração das linhas de carga, e o vetor $\left\{s_{i}\right\}$ contém o valor das cargas em cada nó interno. Em 2.252 o número de equações é igual ao número de incógnitas, portanto é possível resolver o sistema e encontrar todos os valores de contorno. Entretanto, para que a formulação do MEC possa ser acoplada à formulação do MEF no Capítulo 5, é necessário que 2.222 também seja escrita para todos nós definidos ao longo das linhas de 
carga. Este procedimento fornece:

$$
\left\{u_{i}\right\}+\left[H_{i c}\right]\left\{u_{c}\right\}=\left[G_{i c}\right]\left\{p_{c}\right\}+\left[M_{i i}\right]\left\{s_{i}\right\}
$$

Um comentário importante deve ser feito a respeito da equação 2.253, antes de combiná-la à expressão 2.252. Neste trabalho, quando a malha do MEC é gerada, o nó de topo das linhas de carga sempre coincide com algum dos nós da malha de superfície. Isto significa que já estão incluídas, em 2.252, as equações escritas para os nós de topo de todas as linhas de carga. Desta forma, é desnecessário incluir estas equações novamente em 2.253, bastando posicionar o ponto fonte nos nós das linhas de carga que são internos ao domínio. Para evitar que este procedimento torne o número de incógnitas superior ao número de equações no sistema final MEC/MEF, neste trabalho as contribuições das cargas nos nós de topo de estaca são igualadas a zero em 2.252. Portanto, as cargas aplicadas nos elementos de contorno que contém os nós de topo das estacas não contribuem nestes mesmos nós.

Por fim, atentando às considerações feitas acima, as expressões 2.252 e 2.253 são combinadas em um único sistema de equações, obtendo:

$$
\left[\begin{array}{ll}
H_{c c} & 0 \\
H_{i c} & I
\end{array}\right]\left\{\begin{array}{l}
u_{c} \\
u_{i}
\end{array}\right\}=\left[\begin{array}{ll}
G_{c c} & M_{c i} \\
G_{i c} & M_{i i}
\end{array}\right]\left\{\begin{array}{c}
p_{c} \\
s_{i}
\end{array}\right\}
$$

em que $[I]$ representa a matriz identidade.

Assim como em 2.252, em 2.254 o número de equações é igual ao de incógnitas e é possível isolar todas as incógnitas no lado esquerdo da igualdade a partir de uma troca de colunas entre as matrizes. O resultado é um sistema de equações lineares, cuja resolução permite obter todos os valores de contorno incógnitos e também todos os deslocamentos em pontos internos.

\subsection{Considerações finais}

Neste Capítulo, a partir de equações advindas da mecânica dos sólidos e de técnicas de resíduos ponderados, deduziu-se a equação integral de contorno denominada identidade de Somigliana. Apresentou-se também as soluções fundamentais de Kelvin de deslocamento e 
força para uma carga unitária aplicada em um meio tridimensional. A partir da identidade de Somigliana e das soluções fundamentais, formulou-se o problema do valor de contorno e foi obtida a equação 2.83, que é o ponto de partida do MEC.

Adotando aproximações lineares para deslocamentos e forças, foi obtido um sistema de equações cuja solução leva aos valores incógnitos nos pontos definidos no contorno. Descreveu-se então o cálculo de deslocamentos e tensões em pontos internos do sólido a partir dos valores de contorno e técnicas de integração analíticas e numéricas.

Ao final do Capítulo foi apresentada a teoria utilizada para considerar a presença de estacas no meio tridimensional, as quais são representadas por linhas de carga. Aproximase as forças no fuste e base empregando-se funções de forma polinomiais, sendo utilizados polinômios do terceiro grau para aproximar as forças horizontais no fuste, polinômios do segundo grau para aproximar as forças verticais cisalhantes do fuste e uma função constante para aproximar a resultante normal à base da estaca. 


\section{Capítulo 3}

\section{Sólidos compostos por subregiões}

\subsection{Introdução}

São apresentadas neste Capítulo duas formulações para a análise de subregiões pelo método dos elementos de contorno. A primeira, apresentada na Seção 3.2, é mais conhecida na literatura, podendo ser consultada em Brebbia e Dominguez (1992). A segunda é tema da Seção 3.3, e emprega uma técnica alternativa para a consideração de subregiões. Esta técnica, aqui desenvolvida para a simulação de sólidos tridimensionais em contato, é apresentada em Venturini (1992) para problemas elásticos e potenciais e em Paiva e Aliabadi (2000) e Paiva e Aliabadi (2004) para a análise de placas.

Ao final do Capítulo, após apresentar como é feita a inclusão de estacas no caso nãohomogêneo, são apresentados dois exemplos nos quais a técnica clássica é comparada à alternativa. É demonstrado que a técnica alternativa é mais vantajosa, dada sua maior precisão. Por este motivo optou-se, neste trabalho, por empregar sempre a técnica alternativa na consideração de sólidos não-homogêneos.

\subsection{Técnica clássica de subregiões}

Considera-se um problema submetido a condições de contorno quaisquer e com duas subregiões de diferentes características físicas em contato, conforme ilustrado na figura 3.1 . A subregião $\Omega_{1}$ tem módulo de elasticidade $E_{1}$ e coeficiente de Poisson $\nu_{1}$, e a subregião $\Omega_{2}$ tem módulo de elasticidade $E_{2}$ e coeficiente de Poisson $\nu_{2}$.

A equação 2.190 pode ser aplicada independentemente para as subregiões $\Omega_{1}$ e $\Omega_{2}$. 


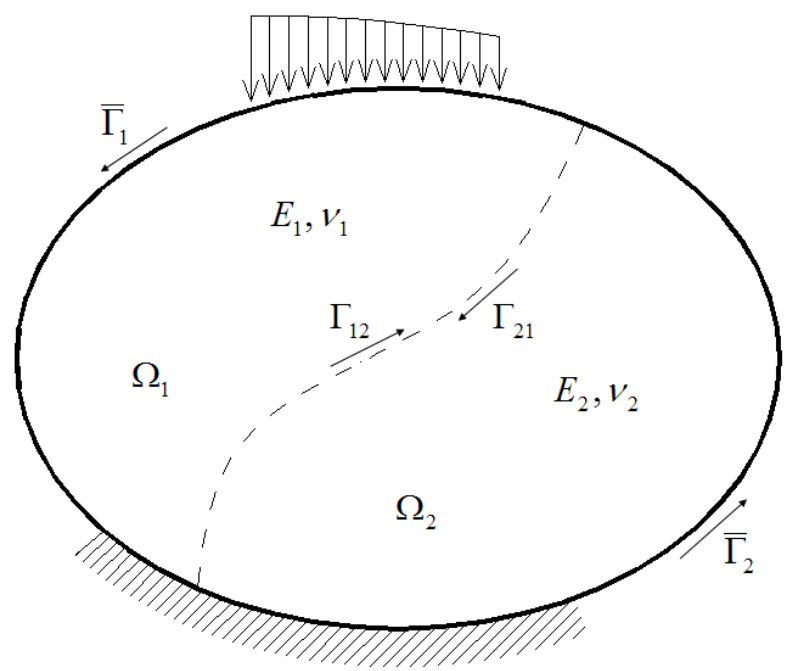

Figura 3.1: Duas subregiões em contato

Assim:

$$
\begin{aligned}
& {\left[H^{1}\right]\left\{u^{1}\right\}=\left[G^{1}\right]\left\{p^{1}\right\}} \\
& {\left[H^{2}\right]\left\{u^{2}\right\}=\left[G^{2}\right]\left\{p^{2}\right\}}
\end{aligned}
$$

Para explicar a forma como é feito o acoplamento torna-se necessário explicitar-se, nas equações 3.1 e 3.2, quais parcelas dos vetores de deslocamentos e forças pertencem ao contato entre as diferentes subregiões. Identificando o contato pela letra $c$ e superfícies livres pela letra $l$, reescreve-se 3.1 e 3.2 como segue:

$$
\begin{gathered}
{\left[\begin{array}{ll}
H_{l l}^{1} & H_{l c}^{1} \\
H_{c l}^{1} & H_{c c}^{1}
\end{array}\right]\left\{\begin{array}{l}
u_{l}^{1} \\
u_{c}^{1}
\end{array}\right\}=\left[\begin{array}{ll}
G_{l l}^{1} & G_{l c}^{1} \\
G_{c l}^{1} & G_{c c}^{1}
\end{array}\right]\left\{\begin{array}{c}
p_{l}^{1} \\
p_{c}^{1}
\end{array}\right\}} \\
{\left[\begin{array}{ll}
H_{l l}^{2} & H_{l c}^{2} \\
H_{c l}^{2} & H_{c c}^{2}
\end{array}\right]\left\{\begin{array}{l}
u_{l}^{2} \\
u_{c}^{2}
\end{array}\right\}=\left[\begin{array}{ll}
G_{l l}^{2} & G_{l c}^{2} \\
G_{c l}^{2} & G_{c c}^{2}
\end{array}\right]\left\{\begin{array}{c}
p_{l}^{2} \\
p_{c}^{2}
\end{array}\right\}}
\end{gathered}
$$

Para acoplar as duas subregiões, são impostas condições de compatibilidade de deslocamentos e equilíbrio de forças na área de contato entre os dois sólidos. Estas condições são expressas pelas igualdades 3.5 e 3.6 :

$$
\begin{gathered}
\left\{u_{c}^{1}\right\}=\left\{u_{c}^{2}\right\}=\left\{u_{c}\right\} \\
\left\{p_{c}^{2}\right\}=-\left\{p_{c}^{1}\right\}=\left\{p_{c}\right\}
\end{gathered}
$$

Partindo das equações 3.3 e 3.4 e tendo em conta as condições 3.5 e 3.6, torna-se 
possível montar um único sistema de equações para representar ambos os sólidos. Este sistema é:

$$
\left[\begin{array}{ccc}
H_{l l}^{2} & H_{l c}^{2} & 0 \\
H_{c l}^{2} & H_{c c}^{2} & 0 \\
0 & H_{c c}^{1} & H_{c l}^{1} \\
0 & H_{l c}^{1} & H_{l l}^{1}
\end{array}\right]\left\{\begin{array}{l}
u_{l}^{2} \\
u_{c} \\
u_{l}^{1}
\end{array}\right\}=\left[\begin{array}{ccc}
G_{l l}^{2} & G_{l c}^{2} & 0 \\
G_{c l}^{2} & G_{c c}^{2} & 0 \\
0 & -G_{c c}^{1} & -G_{c l}^{1} \\
0 & G_{l c}^{1} & G_{l l}^{1}
\end{array}\right]\left\{\begin{array}{c}
p_{l}^{2} \\
p_{c} \\
p_{l}^{1}
\end{array}\right\}
$$

Nota-se que, no lado direito da igualdade, alguns termos da matriz aparecem com sinal negativo. Isto acontece por causa do sinal negativo presente nas condições de equilíbrio expressadas em 3.6 .

Para determinar os termos incógnitos do sistema de equações 3.7 é necessário primeiramente separá-los dos conhecidos. O procedimento a ser adotado é o mesmo feito quando se considera uma única região, conforme explicado entre as equações 2.190 e 2.192 . O resultado neste caso também é um sistema de equações lineares, cujo resultado leva a todos os valores de contorno incógnitos nas superfícies externas e nos contatos. Sendo $n_{1}$ a soma de todos os pontos pertencentes aos contornos externos das duas subregiões e $n_{2}$ a soma dos pontos pertencentes ao contato, o número de incógnitas $n$ do sistema final de equações é, para o caso tridimensional:

$$
n=3 n_{1}+6 n_{2}
$$

Nos pontos pertencentes aos contornos externos, dada uma direção, sempre haverá um valor de contorno incógnito e um conhecido. Já para pontos pertencentes aos contatos, tanto o deslocamento como a força são desconhecidos nas três direções.

Caso se queira analisar um problema geral com um número qualquer de subregiões o raciocínio é o mesmo, bastando aplicar as condições 3.5 e 3.6 em todas as superfícies de contato. A equação 3.8, para cálculo do número de incógnitas do sistema final, também é válida.

\subsection{Técnica alternativa de subregiões}

Nesta Seção, é deduzida uma formulação alternativa para o cálculo de problemas que envolvem subregiões. A formulação, conforme apresentado mais adiante, não emprega relações de compatibilidade de deslocamentos e equilíbrio de forças nas superfícies de 
contato entre as diferentes subregiões como é feito na Seção anterior, e resulta em um sistema de equações com menos incógnitas. A dedução é feita inicialmente para um problema com três subregiões, número a partir do qual surgem todos os termos a serem considerados quando a formulação é estendida a um número qualquer delas. Assim, a figura 3.2 apresenta um domínio submetido a determinadas condições de contorno e dividido em três subregiões de diferentes características físicas

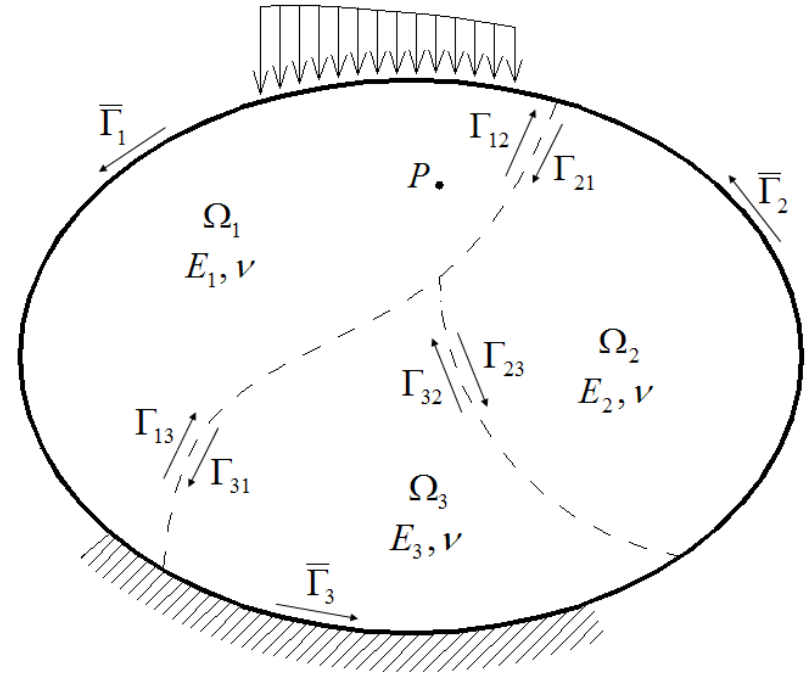

Figura 3.2: Três subregiões em contato

$\mathrm{O}$ ponto $P$ é interno à subregião $\Omega_{1}$ e externo às subregiões $\Omega_{2}$ e $\Omega_{3}$, conforme pode ser visualizado na figura. O módulo de elasticidade de $\Omega_{1}$ é $E_{1}$, e seu contorno $\Gamma_{1}$ está dividido em $\bar{\Gamma}_{1}, \Gamma_{12}$ e $\Gamma_{13}$. A parcela $\Gamma_{12}$ corresponde ao contato com $\Omega_{2}, \Gamma_{13}$ é o contato com $\Omega_{3}$ e o restante de $\Gamma_{1}$ é denominado por $\bar{\Gamma}_{1}$. De forma semelhante, $\Omega_{2}$ tem módulo de elasticidade $E_{2}$ e seu contorno $\Gamma_{2}$ está dividido em $\bar{\Gamma}_{2}, \Gamma_{21}$ e $\Gamma_{23}$, e $\Omega_{3}$ tem módulo de elasticidade $E_{3}$ e seu contorno $\Gamma_{3}$ está dividido em $\bar{\Gamma}_{3}, \Gamma_{31}$ e $\Gamma_{32}$. As três subregiões possuem mesmo coeficiente de Poisson, $\nu$.

Inicialmente, escreve-se a solução fundamental de deslocamento das subregiões $\Omega_{1}, \Omega_{2}$ e $\Omega_{3}$ aplicando-se a equação 2.49 , ou seja:

$$
\begin{aligned}
& u_{1 l k}^{*}=\frac{1}{16 \pi \mu_{1}(1-\nu) r}\left[(3-4 \nu) \delta_{l k}+r_{, l} r, k\right] \\
& u_{2 l k}^{*}=\frac{1}{16 \pi \mu_{2}(1-\nu) r}\left[(3-4 \nu) \delta_{l k}+r_{, l} r, k\right] \\
& u_{3 l k}^{*}=\frac{1}{16 \pi \mu_{3}(1-\nu) r}\left[(3-4 \nu) \delta_{l k}+r_{, l} r, k\right]
\end{aligned}
$$


em que

$$
\mu_{i}=\frac{E_{i}}{2(1+\nu)}
$$

A partir das equações 3.9, 3.10 e 3.11, e observando a igualdade 3.12, as seguintes relações podem ser obtidas entre as soluções fundamentais:

$$
\begin{aligned}
& u_{2 l k}^{*}=\frac{E_{1}}{E_{2}} u_{1 l k}^{*} \\
& u_{3 l k}^{*}=\frac{E_{1}}{E_{3}} u_{1 l k}^{*}
\end{aligned}
$$

As expressões 3.13 e 3.14 podem ser reescritas como:

$$
\begin{aligned}
& u_{2 l k}^{*}=u_{1 l k}^{*}+\frac{\Delta E_{12}}{E_{1}} u_{2 l k}^{*} \\
& u_{3 l k}^{*}=u_{1 l k}^{*}+\frac{\Delta E_{13}}{E_{1}} u_{3 l k}^{*}
\end{aligned}
$$

em que:

$$
\Delta E_{i j}=\left(E_{i}-E_{j}\right)
$$

Para analisar as soluções fundamentais de força dos domínios $\Omega_{1}, \Omega_{2}$ e $\Omega_{3}$ recorre-se à expressão 2.54, que é:

$$
p_{l k}^{*}=\frac{-1}{8 \pi(1-\nu) r^{2}}\left[\frac{\partial r}{\partial \eta}\left[(1-2 \nu) \delta_{l k}+3 r,{ }_{l} r_{, k}\right]+(1-2 \nu)\left(\eta_{l} r_{, k}-\eta_{k} r_{, l}\right)\right]
$$

Nota-se que a expressão não contém o módulo de elasticidade e é portanto a mesma para os domínios $\Omega_{1}, \Omega_{2}$ e $\Omega_{3}$, ou seja, $p_{1 l k}^{*}=p_{2 l k}^{*}=p_{3 l k}^{*}=p_{l k}^{*}$. Esta conclusão, em conjunto com as igualdades 3.15 e 3.16, são relações necessárias para as deduções apresentadas a seguir.

Seja um ponto $P$ qualquer posicionado no interior da subregião $\Omega_{1}$, conforme ilustrado na figura 3.2. Igualando as cargas de domínio a zero, escreve-se a equação 2.83 para o ponto $P$ considerando a subregião $\Omega_{1}$, ou seja:

$$
c_{1 l k}^{i} u_{l}^{i}+\int_{\Gamma_{1}} p_{l k}^{*} u_{k} d \Gamma_{1}=\int_{\Gamma_{1}} u_{1 l k}^{*} p_{k} d \Gamma_{1}
$$

É importante que sejam feitos alguns comentários a respeito desta equação. Nota-se, 
primeiramente, que a solução fundamental de força da subregião $\Omega_{1}$ foi denominada por $p_{l k}^{*}$ de forma geral, pois ela é a mesma para as subregiões $\Omega_{2}$ e $\Omega_{3}$. Em segundo lugar, mesmo estando o ponto $P$ contido na subregião $\Omega_{1}$, considerou-se o caso mais geral no qual deslocamento $u_{l}^{i}$ aparece multiplicado pelo termo $c_{1 l k}^{i}$. Isto é necessário para que posteriormente seja possível considerar o ponto fonte sobre o contorno.

O passo seguinte é reescrever a equação 3.18 separando a parcela do contorno pertencente aos contatos, $\Gamma_{12}$ e $\Gamma_{13}$, da não pertencente aos contatos, $\bar{\Gamma}_{1}$. Assim:

$$
\begin{gathered}
c_{1 l k}^{i} u_{l}^{i}+\int_{\bar{\Gamma}_{1}} p_{l k}^{*} u_{k} d \bar{\Gamma}_{1}+\int_{\Gamma_{12}} p_{l k}^{*} u_{k} d \Gamma_{12}+\int_{\Gamma_{13}} p_{l k}^{*} u_{k} d \Gamma_{13}= \\
\int_{\bar{\Gamma}_{1}} u_{1 l k}^{*} p_{k} d \bar{\Gamma}_{1}+\int_{\Gamma_{12}} u_{1 l k}^{*} p_{k} d \Gamma_{12}+\int_{\Gamma_{13}} u_{1 l k}^{*} p_{k} d \Gamma_{13}
\end{gathered}
$$

Também é possível escrever a equação 2.83 para o ponto $P$ mas integrando o contorno da subregião $\Omega_{2}$, ou seja:

$$
\begin{gathered}
c_{2 l k}^{i} u_{l}^{i}+\int_{\bar{\Gamma}_{2}} p_{l k}^{*} u_{k} d \bar{\Gamma}_{2}+\int_{\Gamma_{21}} p_{l k}^{*} u_{k} d \Gamma_{21}+\int_{\Gamma_{23}} p_{l k}^{*} u_{k} d \Gamma_{23}= \\
\int_{\bar{\Gamma}_{2}} u_{2 l k}^{*} p_{k} d \bar{\Gamma}_{2}+\int_{\Gamma_{21}} u_{2 l k}^{*} p_{k} d \Gamma_{21}+\int_{\Gamma_{23}} u_{2 l k}^{*} p_{k} d \Gamma_{23}
\end{gathered}
$$

O mesmo vale para a subregião $\Omega_{3}$ :

$$
\begin{gathered}
c_{3 l k}^{i} u_{l}^{i}+\int_{\bar{\Gamma}_{3}} p_{l k}^{*} u_{k} d \bar{\Gamma}_{3}+\int_{\Gamma_{31}} p_{l k}^{*} u_{k} d \Gamma_{31}+\int_{\Gamma_{32}} p_{l k}^{*} u_{k} d \Gamma_{32}= \\
\int_{\bar{\Gamma}_{3}} u_{3 l k}^{*} p_{k} d \bar{\Gamma}_{3}+\int_{\Gamma_{31}} u_{3 l k}^{*} p_{k} d \Gamma_{31}+\int_{\Gamma_{32}} u_{3 l k}^{*} p_{k} d \Gamma_{32}
\end{gathered}
$$

Ao somar as equações 3.19 , 3.20 e 3.21 obtém-se uma única equação para o ponto $P$, sendo que esta equação considera a influência das três subregiões. Assim:

$$
\begin{gathered}
\left(c_{1 l k}^{i}+c_{2 l k}^{i}+c_{3 l k}^{i}\right) u_{l}^{i}+\int_{\bar{\Gamma}_{1}} p_{l k}^{*} u_{k} d \bar{\Gamma}_{1}+\int_{\Gamma_{12}} p_{l k}^{*} u_{k} d \Gamma_{12}+\int_{\Gamma_{13}} p_{l k}^{*} u_{k} d \Gamma_{13}+\int_{\bar{\Gamma}_{2}} p_{l k}^{*} u_{k} d \bar{\Gamma}_{2}+ \\
+\int_{\Gamma_{21}} p_{l k}^{*} u_{k} d \Gamma_{21}+\int_{\Gamma_{23}} p_{l k}^{*} u_{k} d \Gamma_{23}+\int_{\bar{\Gamma}_{3}} p_{l k}^{*} u_{k} d \bar{\Gamma}_{3}+\int_{\Gamma_{31}} p_{l k}^{*} u_{k} d \Gamma_{31}+\int_{\Gamma_{32}} p_{l k}^{*} u_{k} d \Gamma_{32}= \\
=\int_{\bar{\Gamma}_{1}} u_{1 l k}^{*} p_{k} d \bar{\Gamma}_{1}+\int_{\Gamma_{12}} u_{1 l k}^{*} p_{k} d \Gamma_{12}+\int_{\Gamma_{13}} u_{1 l k}^{*} p_{k} d \Gamma_{13}+\int_{\bar{\Gamma}_{2}} u_{2 l k}^{*} p_{k} d \bar{\Gamma}_{2}+\int_{\Gamma_{21}} u_{2 l k}^{*} p_{k} d \Gamma_{21}+ \\
\quad+\int_{\Gamma_{23}} u_{2 l k}^{*} p_{k} d \Gamma_{23}+\int_{\bar{\Gamma}_{3}} u_{3 l k}^{*} p_{k} d \bar{\Gamma}_{3}+\int_{\Gamma_{31}} u_{3 l k}^{*} p_{k} d \Gamma_{31}+\int_{\Gamma_{32}} u_{3 l k}^{*} p_{k} d \Gamma_{32}
\end{gathered}
$$

É possível reduzir o número de termos desta equação analisando como estes termos se relacionam. Iniciando com o lado esquerdo da igualdade, conclui-se que a seguinte relação 
é verdadeira:

$$
\int_{\Gamma_{12}} p_{l k}^{*} u_{k} d \Gamma_{12}+\int_{\Gamma_{21}} p_{l k}^{*} u_{k} d \Gamma_{21}=0
$$

A justificativa do porquê desta soma ser igual a zero reside no fato de ser exatamente a mesma função que está sendo integrada nos dois termos. A única diferença é o sentido da integração, conforme indicado por setas na figura 3.2. Ao inverter o sentido de integração o valor absoluto da integral não é alterado, mas seu sinal é trocado porque o vetor normal fica com o sentido oposto. Tendo os dois termos o mesmo valor absoluto e sinais diferentes, sua soma é zero. Apesar do desenho da figura 3.2 ser bidimensional o mesmo vale para o caso tridimensional, pois ocorre a mesma inversão do vetor normal.

Pelo mesmo motivo, são verdadeiras as igualdades:

$$
\begin{aligned}
& \int_{\Gamma_{13}} p_{l k}^{*} u_{k} d \Gamma_{13}+\int_{\Gamma_{31}} p_{l k}^{*} u_{k} d \Gamma_{31}=0 \\
& \int_{\Gamma_{23}} p_{l k}^{*} u_{k} d \Gamma_{23}+\int_{\Gamma_{32}} p_{l k}^{*} u_{k} d \Gamma_{32}=0
\end{aligned}
$$

É possível simplificar também o lado direito da equação 3.22 . Para isto, é necessário substituir as expressões 3.15 e 3.16 em alguns dos termos. Inicia-se com o termo abaixo:

$$
\int_{\Gamma_{21}} u_{2 l k}^{*} p_{k} d \Gamma_{21}=\int_{\Gamma_{21}}\left(u_{1 l k}^{*}+\frac{\Delta E_{12}}{E_{1}} u_{2 l k}^{*}\right) p_{k} d \Gamma_{21}=\int_{\Gamma_{21}} u_{1 l k}^{*} p_{k} d \Gamma_{21}+\frac{\Delta E_{12}}{E_{1}} \int_{\Gamma_{21}} u_{2 l k}^{*} p_{k} d \Gamma_{21}
$$

Observando a expressão à direita da segunda igualdade em 3.26, nota-se que é possível relacioná-la com um termo da equação 3.22 de uma forma semelhante à feita na relação 3.23. Ou seja:

$$
\int_{\Gamma_{12}} u_{1 l k}^{*} p_{k} d \Gamma_{12}+\int_{\Gamma_{21}} u_{2 l k}^{*} p_{k} d \Gamma_{21}=\left(\int_{\Gamma_{12}} u_{1 l k}^{*} p_{k} d \Gamma_{12}+\int_{\Gamma_{21}} u_{1 l k}^{*} p_{k} d \Gamma_{21}\right)+\frac{\Delta E_{12}}{E_{1}} \int_{\Gamma_{21}} u_{2 l k}^{*} p_{k} d \Gamma_{21}
$$

O termo entre parênteses no lado direito da igualdade 3.27 é igual a zero pelo mesmo motivo que a soma 3.23 é igual a zero. Assim, chega-se à expressão:

$$
\int_{\Gamma_{12}} u_{1 l k}^{*} p_{k} d \Gamma_{12}+\int_{\Gamma_{21}} u_{2 l k}^{*} p_{k} d \Gamma_{21}=\frac{\Delta E_{12}}{E_{1}} \int_{\Gamma_{21}} u_{2 l k}^{*} p_{k} d \Gamma_{21}
$$


Analogamente, escreve-se também:

$$
\int_{\Gamma_{13}} u_{1 l k}^{*} p_{k} d \Gamma_{13}+\int_{\Gamma_{31}} u_{3 l k}^{*} p_{k} d \Gamma_{31}=\frac{\Delta E_{13}}{E_{1}} \int_{\Gamma_{31}} u_{3 l k}^{*} p_{k} d \Gamma_{31}
$$

Também é possível relacionar as integrais sobre os contornos $\Gamma_{23}$ e $\Gamma_{32}$ substituindo-se nestes termos as expressões 3.15 e 3.16, conforme demonstrado abaixo:

$$
\begin{gathered}
\int_{\Gamma_{23}} u_{2 l k}^{*} p_{k} d \Gamma_{23}+\int_{\Gamma_{32}} u_{3 l k}^{*} p_{k} d \Gamma_{32}=\int_{\Gamma_{23}}\left(u_{1 l k}^{*}+\frac{\Delta E_{12}}{E_{1}} u_{2 l k}^{*}\right) p_{k} d \Gamma_{23}+ \\
+\int_{\Gamma_{32}}\left(u_{1 l k}^{*}+\frac{\Delta E_{13}}{E_{1}} u_{3 l k}^{*}\right) p_{k} d \Gamma_{32}=\left(\int_{\Gamma_{23}} u_{1 l k}^{*} p_{k} d \Gamma_{23}+\int_{\Gamma_{32}} u_{1 l k}^{*} p_{k} d \Gamma_{32}\right)+\frac{\Delta E_{12}}{E_{1}} \int_{\Gamma_{23}} u_{2 l k}^{*} p_{k} d \Gamma_{23}+ \\
+\frac{\Delta E_{13}}{E_{1}} \int_{\Gamma_{32}} u_{3 l k}^{*} p_{k} d \Gamma_{32}
\end{gathered}
$$

Como a soma de integrais no parênteses à direita da segunda igualdade é zero, chega-se à relação:

$$
\int_{\Gamma_{23}} u_{2 l k}^{*} p_{k} d \Gamma_{23}+\int_{\Gamma_{32}} u_{3 l k}^{*} p_{k} d \Gamma_{32}=\frac{\Delta E_{12}}{E_{1}} \int_{\Gamma_{23}} u_{2 l k}^{*} p_{k} d \Gamma_{23}+\frac{\Delta E_{13}}{E_{1}} \int_{\Gamma_{32}} u_{3 l k}^{*} p_{k} d \Gamma_{32}
$$

Mais uma simplificação pode ser feita no lado direito da equação 3.22 caso a expressão 3.15 seja substituída em mais um termo, conforme demonstrado abaixo:

$$
\int_{\bar{\Gamma}_{2}} u_{2 l k}^{*} p_{k} d \bar{\Gamma}_{2}=\int_{\bar{\Gamma}_{2}}\left(u_{1 l k}^{*}+\frac{\Delta E_{12}}{E_{1}} u_{2 l k}^{*}\right) p_{k} d \bar{\Gamma}_{2}=\int_{\bar{\Gamma}_{2}} u_{1 l k}^{*} p_{k} d \bar{\Gamma}_{2}+\frac{\Delta E_{12}}{E_{1}} \int_{\bar{\Gamma}_{2}} u_{2 l k}^{*} p_{k} d \bar{\Gamma}_{2}
$$

Analogamente à relação 3.32

$$
\int_{\bar{\Gamma}_{3}} u_{3 l k}^{*} p_{k} d \bar{\Gamma}_{3}=\int_{\bar{\Gamma}_{3}} u_{1 l k}^{*} p_{k} d \bar{\Gamma}_{3}+\frac{\Delta E_{13}}{E_{1}} \int_{\bar{\Gamma}_{3}} u_{3 l k}^{*} p_{k} d \bar{\Gamma}_{3}
$$

Após estas deduções, substitui-se em 3.22 as expressões 3.23, 3.24, 3.25, 3.28, 3.29, 3.31 , 3.32 e 3.33. O resultado é a seguinte equação:

$$
\begin{gathered}
\left(c_{1 l k}^{i}+c_{2 l k}^{i}+c_{3 l k}^{i}\right) u_{l}^{i}+\int_{\bar{\Gamma}_{1}} p_{l k}^{*} u_{k} d \bar{\Gamma}_{1}+\int_{\bar{\Gamma}_{2}} p_{l k}^{*} u_{k} d \bar{\Gamma}_{2}+\int_{\bar{\Gamma}_{3}} p_{l k}^{*} u_{k} d \bar{\Gamma}_{3}=\int_{\bar{\Gamma}_{1}} u_{1 l k}^{*} p_{k} d \bar{\Gamma}_{1}+ \\
+\int_{\bar{\Gamma}_{2}} u_{1 l k}^{*} p_{k} d \bar{\Gamma}_{2}+\frac{\Delta E_{12}}{E_{1}} \int_{\bar{\Gamma}_{2}} u_{2 l k}^{*} p_{k} d \bar{\Gamma}_{2}+\int_{\bar{\Gamma}_{3}} u_{1 l k}^{*} p_{k} d \bar{\Gamma}_{3}+\frac{\Delta E_{13}}{E_{1}} \int_{\bar{\Gamma}_{3}} u_{3 l k}^{*} p_{k} d \bar{\Gamma}_{3}+ \\
+\frac{\Delta E_{12}}{E_{1}} \int_{\Gamma_{21}} u_{2 l k}^{*} p_{k} d \Gamma_{21}+\frac{\Delta E_{13}}{E_{1}} \int_{\Gamma_{31}} u_{3 l k}^{*} p_{k} d \Gamma_{31}+\frac{\Delta E_{12}}{E_{1}} \int_{\Gamma_{23}} u_{2 l k}^{*} p_{k} d \Gamma_{23}+\frac{\Delta E_{13}}{E_{1}} \int_{\Gamma_{32}} u_{3 l k}^{*} p_{k} d \Gamma_{32}
\end{gathered}
$$


Reorganizando os termos da igualdade 3.34 , obtém-se:

$$
\begin{aligned}
\left(c_{1 l k}^{i}+c_{2 l k}^{i}+c_{3 l k}^{i}\right) u_{l}^{i}+\int_{\bar{\Gamma}_{1}} p_{l k}^{*} u_{k} d \bar{\Gamma}_{1}+\int_{\bar{\Gamma}_{2}} p_{l k}^{*} u_{k} d \bar{\Gamma}_{2}+\int_{\bar{\Gamma}_{3}} p_{l k}^{*} u_{k} d \bar{\Gamma}_{3}=\int_{\bar{\Gamma}_{1}} u_{1 l k}^{*} p_{k} d \bar{\Gamma}_{1}+ \\
+\int_{\bar{\Gamma}_{2}} u_{1 l k}^{*} p_{k} d \bar{\Gamma}_{2}+\int_{\bar{\Gamma}_{3}} u_{1 l k}^{*} p_{k} d \bar{\Gamma}_{3}+\frac{\Delta E_{12}}{E_{1}}\left(\int_{\bar{\Gamma}_{2}} u_{2 l k}^{*} p_{k} d \bar{\Gamma}_{2}+\int_{\Gamma_{21}} u_{2 l k}^{*} p_{k} d \Gamma_{21}+\int_{\Gamma_{23}} u_{2 l k}^{*} p_{k} d \Gamma_{23}\right)+ \\
+\frac{\Delta E_{13}}{E_{1}}\left(\int_{\bar{\Gamma}_{3}} u_{3 l k}^{*} p_{k} d \bar{\Gamma}_{3}+\int_{\Gamma_{31}} u_{3 l k}^{*} p_{k} d \Gamma_{31}+\int_{\Gamma_{32}} u_{3 l k}^{*} p_{k} d \Gamma_{32}\right)
\end{aligned}
$$

Os termos entre parênteses são função das forças na interface entre as subregiões, entretanto é mais interessante que eles sejam função dos deslocamentos na interface. Isto é possível recorrendo-se às equações 3.20 e 3.21 , isolando uma de suas integrais para posterior substituição em 3.35 . Ou seja:

$$
\begin{aligned}
\int_{\bar{\Gamma}_{2}} u_{2 l k}^{*} p_{k} d \bar{\Gamma}_{2}=c_{2 l k}^{i} u_{l}^{i}+\int_{\bar{\Gamma}_{2}} p_{l k}^{*} u_{k} d \bar{\Gamma}_{2} & +\int_{\Gamma_{21}} p_{l k}^{*} u_{k} d \Gamma_{21}+\int_{\Gamma_{23}} p_{l k}^{*} u_{k} d \Gamma_{23}-\int_{\Gamma_{21}} u_{2 l k}^{*} p_{k} d \Gamma_{21}+ \\
& -\int_{\Gamma_{23}} u_{2 l k}^{*} p_{k} d \Gamma_{23} \\
\int_{\bar{\Gamma}_{3}} u_{3 l k}^{*} p_{k} d \bar{\Gamma}_{3}=c_{3 l k}^{i} u_{l}^{i}+\int_{\Gamma_{31}} p_{l k}^{*} u_{k} d \Gamma_{31} & +\int_{\Gamma_{32}} p_{l k}^{*} u_{k} d \Gamma_{32}+\int_{\bar{\Gamma}_{3}} p_{l k}^{*} u_{k} d \bar{\Gamma}_{3}-\int_{\Gamma_{31}} u_{3 l k}^{*} p_{k} d \Gamma_{31}+ \\
& -\int_{\Gamma_{32}} u_{3 l k}^{*} p_{k} d \Gamma_{32}
\end{aligned}
$$

Substituindo então 3.36 e 3.37 em 3.35 e combinando as integrais nos parênteses, obtém-se:

$$
\begin{gathered}
\left(c_{1 l k}^{i}+c_{2 l k}^{i}+c_{3 l k}^{i}\right) u_{l}^{i}+\int_{\bar{\Gamma}_{1}} p_{l k}^{*} u_{k} d \bar{\Gamma}_{1}+\int_{\bar{\Gamma}_{2}} p_{l k}^{*} u_{k} d \bar{\Gamma}_{2}+\int_{\bar{\Gamma}_{3}} p_{l k}^{*} u_{k} d \bar{\Gamma}_{3}= \\
=\frac{\Delta E_{12}}{E_{1}}\left(\int_{\bar{\Gamma}_{2}} p_{l k}^{*} u_{k} d \bar{\Gamma}_{2}+\int_{\Gamma_{21}} p_{l k}^{*} u_{k} d \Gamma_{21}+\int_{\Gamma_{23}} p_{l k}^{*} u_{k} d \Gamma_{23}+c_{2 l k}^{i} u_{l}^{i}\right)+\int_{\bar{\Gamma}_{1}} u_{1 l k}^{*} p_{k} d \bar{\Gamma}_{1}+ \\
+\int_{\bar{\Gamma}_{2}} u_{1 l k}^{*} p_{k} d \bar{\Gamma}_{2}+\int_{\bar{\Gamma}_{3}} u_{1 l k}^{*} p_{k} d \bar{\Gamma}_{3}+\frac{\Delta E_{13}}{E_{1}}\left(\int_{\bar{\Gamma}_{3}} p_{l k}^{*} u_{k} d \bar{\Gamma}_{3}+\int_{\Gamma_{31}} p_{l k}^{*} u_{k} d \Gamma_{31}+\iint_{\Gamma_{32}} p_{l k}^{*} u_{k} d \Gamma_{32}+c_{3 l k}^{i} u_{l}^{i}\right) \\
{\left[c_{1 l k}^{i}+c_{2 l k}^{i}\left(1-\frac{\Delta E_{12}}{E_{1}}\right)+c_{3 l k}^{i}\left(1-\frac{\Delta E_{13}}{E_{1}}\right)\right] u_{l}^{i}+\int_{\bar{\Gamma}_{1}} p_{l k}^{*} u_{k} d \bar{\Gamma}_{1}+\int_{\bar{\Gamma}_{2}} p_{l k}^{*} u_{k} d \bar{\Gamma}_{2}+\int_{\bar{\Gamma}_{3}} p_{l k}^{*} u_{k} d \bar{\Gamma}_{3}+} \\
-\frac{\Delta E_{12}}{E_{1}}\left(\int_{\bar{\Gamma}_{2}} p_{l k}^{*} u_{k} d \bar{\Gamma}_{2}+\int_{\Gamma_{21}} p_{l k}^{*} u_{k} d \Gamma_{21}+\int_{\Gamma_{23}} p_{l k}^{*} u_{k} d \Gamma_{23}\right)-\frac{\Delta E_{13}}{E_{1}}\left(\int_{\bar{\Gamma}_{3}} p_{l k}^{*} u_{k} d \bar{\Gamma}_{3}+\right. \\
\left.+\int_{\Gamma_{31}} p_{l k}^{*} u_{k} d \Gamma_{31}+\int_{\Gamma_{32}} p_{l k}^{*} u_{k} d \Gamma_{32}\right)=\int_{\bar{\Gamma}_{1}} u_{1 l k}^{*} p_{k} d \bar{\Gamma}_{1}+\int_{\bar{\Gamma}_{2}} u_{1 l k}^{*} p_{k} d \bar{\Gamma}_{2}+\int_{\bar{\Gamma}_{3}} u_{1 l k}^{*} p_{k} d \bar{\Gamma}_{3}
\end{gathered}
$$

Nota-se que é possível combinar algumas das integrais no lado esquerdo da igualdade 
3.39. Tomando inicialmente as integrais sobre o contorno $\bar{\Gamma}_{2}$, conclui-se que:

$$
\int_{\bar{\Gamma}_{2}} p_{l k}^{*} u_{k} d \bar{\Gamma}_{2}-\frac{\Delta E_{12}}{E_{1}} \int_{\bar{\Gamma}_{2}} p_{l k}^{*} u_{k} d \bar{\Gamma}_{2}=\left(1-\frac{\Delta E_{12}}{E_{1}}\right) \int_{\bar{\Gamma}_{2}} p_{l k}^{*} u_{k} d \bar{\Gamma}_{2}=\frac{E_{2}}{E_{1}} \int_{\bar{\Gamma}_{2}} p_{l k}^{*} u_{k} d \bar{\Gamma}_{2}
$$

Da mesma forma:

$$
\int_{\bar{\Gamma}_{3}} p_{l k}^{*} u_{k} d \bar{\Gamma}_{3}-\frac{\Delta E_{13}}{E_{1}} \int_{\bar{\Gamma}_{3}} p_{l k}^{*} u_{k} d \bar{\Gamma}_{3}=\left(1-\frac{\Delta E_{13}}{E_{1}}\right) \int_{\bar{\Gamma}_{3}} p_{l k}^{*} u_{k} d \bar{\Gamma}_{3}=\frac{E_{3}}{E_{1}} \int_{\bar{\Gamma}_{3}} p_{l k}^{*} u_{k} d \bar{\Gamma}_{3}
$$

Também é possível relacionar as integrais sobre os contornos $\Gamma_{23}$ e $\Gamma_{32}$ uma com a outra, da seguinte forma:

$$
\begin{aligned}
& \frac{\Delta E_{12}}{E_{1}} \int_{\Gamma_{23}} p_{l k}^{*} u_{k} d \Gamma_{23}+\frac{\Delta E_{13}}{E_{1}} \int_{\Gamma_{32}} p_{l k}^{*} u_{k} d \Gamma_{32}=\frac{\Delta E_{12}}{E_{1}} \int_{\Gamma_{23}} p_{l k}^{*} u_{k} d \Gamma_{23}-\frac{\Delta E_{13}}{E_{1}} \int_{\Gamma_{23}} p_{l k}^{*} u_{k} d \Gamma_{23}= \\
& \left(\frac{\Delta E_{12}}{E_{1}}-\frac{\Delta E_{13}}{E_{1}}\right) \int_{\Gamma_{23}} p_{l k}^{*} u_{k} d \Gamma_{23}=\left(\frac{E_{1}-E_{2}}{E_{1}}-\frac{E_{1}-E_{3}}{E_{1}}\right) \int_{\Gamma_{23}} p_{l k}^{*} u_{k} d \Gamma_{23}=\frac{E_{3}-E_{2}}{E_{1}} \int_{\Gamma_{23}} p_{l k}^{*} u_{k} d \Gamma_{23}
\end{aligned}
$$

Empregando a expressão 3.17, chega-se à relação:

$$
\frac{\Delta E_{12}}{E_{1}} \int_{\Gamma_{23}} p_{l k}^{*} u_{k} d \Gamma_{23}+\frac{\Delta E_{13}}{E_{1}} \int_{\Gamma_{32}} p_{l k}^{*} u_{k} d \Gamma_{32}=\frac{\Delta E_{32}}{E_{1}} \int_{\Gamma_{23}} p_{l k}^{*} u_{k} d \Gamma_{23}
$$

Finalmente, substitui-se as expressões 3.40, 3.41 e 3.43 em 3.39, chegando à seguinte equação:

$$
\begin{gathered}
{\left[c_{1 l k}^{i}+c_{2 l k}^{i}\left(\frac{E_{2}}{E_{1}}\right)+c_{3 l k}^{i}\left(\frac{E_{3}}{E_{1}}\right)\right] u_{l}^{i}+\int_{\bar{\Gamma}_{1}} p_{l k}^{*} u_{k} d \bar{\Gamma}_{1}+\frac{E_{2}}{E_{1}} \int_{\bar{\Gamma}_{2}} p_{l k}^{*} u_{k} d \bar{\Gamma}_{2}+} \\
+\frac{E_{3}}{E_{1}} \int_{\bar{\Gamma}_{3}} p_{l k}^{*} u_{k} d \bar{\Gamma}_{3}-\frac{\Delta E_{12}}{E_{1}} \int_{\Gamma_{21}} p_{l k}^{*} u_{k} d \Gamma_{21}-\frac{\Delta E_{13}}{E_{1}} \int_{\Gamma_{31}} p_{l k}^{*} u_{k} d \Gamma_{31}-\frac{\Delta E_{32}}{E_{1}} \int_{\Gamma_{23}} p_{l k}^{*} u_{k} d \Gamma_{23}= \\
=\int_{\bar{\Gamma}_{1}} u_{1 l k}^{*} p_{k} d \bar{\Gamma}_{1}+\int_{\bar{\Gamma}_{2}} u_{1 l k}^{*} p_{k} d \bar{\Gamma}_{2}+\int_{\bar{\Gamma}_{3}} u_{1 l k}^{*} p_{k} d \bar{\Gamma}_{3}
\end{gathered}
$$

Observando a equação 3.44, obtida para três subdomínios, conclui-se que a expressão final válida para um número qualquer de subdomínios é:

$$
\begin{gathered}
\left\{\sum_{s=1}^{n s}\left[\frac{E_{s}}{E_{1}} c_{s l k}^{i}\right]\right\} u_{l}^{i}+\sum_{e=1}^{n e}\left[\frac{E_{e}}{E_{1}} \int_{\bar{\Gamma}_{e}} p_{l k}^{*} u_{k} d \bar{\Gamma}_{e}\right]+\sum_{c=1}^{n c}\left[\frac{\Delta E_{m n}}{E_{1}} \int_{\Gamma_{m n}} p_{l k}^{*} u_{k} d \Gamma_{m n}\right]= \\
=\sum_{e=1}^{n e}\left[\int_{\bar{\Gamma}_{e}} u_{1 l k}^{*} p_{k} d \bar{\Gamma}_{e}\right]
\end{gathered}
$$

Em 3.45, o sinal das integrais do segundo somatório aparece positivo porque o sentido 
de integração foi alterado de $\Gamma_{n m}$ para $\Gamma_{m n}$. Iniciando com o primeiro somatório à esquerda da igualdade, ns corresponde ao número total de subregiões definidas no problema. Para definir por qual valor $c_{l k}^{i}$ o deslocamento $u_{l}^{i}$ deve ser multiplicado, deve-se inicialmente determinar o coeficiente $c_{s l k}^{i}$ em relação a cada subregião $s$ em função da posição do ponto fonte. O coeficiente é então obtido pelo cálculo do somatório de 1 até $n s$ definido na equação 3.45. Deve-se observar que a contribuição da subregião $\Omega_{1}$ no somatório é sempre $c_{1 l k}^{i}$, pois $E_{1 / E_{1}}=1$. Considerando todos os contornos suaves e as três subregiões apresentadas na figura 3.2 , a tabela 3.1 apresenta alguns exemplos de como deve ser feito este cálculo.

\begin{tabular}{|c|c|c|c|c|}
\hline Posição do ponto fonte & $\mathbf{c}_{\mathbf{1} \mathbf{k}}^{\mathbf{i}}$ & $\mathbf{c}_{\mathbf{2} \mathbf{k}}^{\mathbf{i}}$ & $\mathbf{c}_{\mathbf{3} \mathbf{k}}^{\mathbf{i}}$ & $\mathbf{c}_{\mathbf{l k}}^{\mathbf{i}}$ \\
\hline Externo a todas as subregiões & 0 & 0 & 0 & 0 \\
\hline Contido em $\Omega_{1}$ & 1 & 0 & 0 & 1 \\
\hline Sobre o contorno $\bar{\Gamma}_{2}$ & 0 & $1 / 2$ & 0 & $E_{2} / 2 E_{1}$ \\
\hline Sobre o contorno $\Gamma_{12}$ & $1 / 2$ & $1 / 2$ & 0 & $\left(E_{1}+E_{2}\right) / 2 E_{1}$ \\
\hline
\end{tabular}

Tabela 3.1: Exemplos do cálculo do coeficiente $c_{l k}^{i}$

Ainda em relação à equação 3.45 , o termo $n_{e}$ corresponde ao número de subregiões que possuem contornos externos $\bar{\Gamma}$, ou seja, que não estão em contato com outras subregiões. Logicamente, caso um determinado subdomínio não possua contorno externo, sua contribuição neste somatório será nula. Observa-se que, para o domínio $\Omega_{1}$, a relação entre módulos de elasticidade que consta nesse somatório é unitária, o que permite englobar também a contribuição de $\Omega_{1}$.

No terceiro somatório, $n_{c}$ é o número total de contornos em contato. Assim, dado um contorno referente ao contato entre duas subregiões $\Omega_{m}$ e $\Omega_{n}$ quaisquer, deve-se empregar os módulos de elasticidade $E_{m}$ e $E_{n}$ para calcular $\Delta E_{m n}$ e então computar a contribuição deste contorno integrando-se sobre $\Gamma_{m n}$.

O somatório que aparece no lado direito da igualdade 3.45 funciona de forma semelhante ao segundo somatório à esquerda, sendo também de 1 a $n_{e}$ e as integrais sobre os mesmos contornos. As diferenças são a inexistência da relação entre módulos de elasticidade e as funções integradas, que neste caso são a solução fundamental de deslocamento da subregião $\Omega_{1}$ multiplicada pelas forças de cada contorno. 


\subsubsection{Sistema de equações}

A partir da expressão 3.45, os procedimentos para chegar ao sistema de equações são semelhantes aos adotados anteriormente para a expressão 2.83. Repetindo as deduções das seções 2.4.1 e 2.5 de forma análoga, obtém-se a expressão:

$$
\mathbf{c}^{i} \mathbf{u}^{i}+\sum_{e=1}^{n e}\left[\frac{E_{e}}{E_{1}} \int_{\bar{\Gamma}_{e}} \mathbf{p}^{*} \mathbf{u} d \bar{\Gamma}_{e}\right]+\sum_{c=1}^{n c}\left[\frac{\Delta E_{m n}}{E_{1}} \int_{\Gamma_{m n}} \mathbf{p}^{*} \mathbf{u} d \Gamma_{m n}\right]=\sum_{e=1}^{n e}\left[\int_{\bar{\Gamma}_{e}} \mathbf{u}_{1}^{*} \mathbf{p} d \bar{\Gamma}_{e}\right]
$$

em que os termos $\mathbf{c}^{i}, \mathbf{u}^{i}, \mathbf{p}^{*}, \mathbf{p}$ e $\mathbf{u}$, escritos em notação matricial, são definidos da mesma forma que na igualdade 2.102, e a matriz $\mathbf{u}_{1}^{*}$ corresponde à solução fundamental de deslocamento da subregião $\Omega_{1}$.

Esta expressão deve ser então escrita para todos os pontos de todas as subregiões, lembrando que basta escrevê-la uma única vez para cada ponto comum a duas subregiões. As integrais são calculadas numericamente ou semi-analiticamente, da mesma forma descrita nos itens 2.5 .1 e 2.5 .2 ,

Analogamente à Seção 2.5.3, chega-se ao seguinte sistema de equações:

$$
\mathbf{H u}=\mathrm{Gp}
$$

Este sistema tem como incógnitas os deslocamentos em todas as interfaces, além dos valores de contorno que não foram prescritos nas superfícies livres. Deve-se atentar ao fato de que a matriz $\mathbf{H}$ resulta quadrada e a matriz $\mathbf{G}$ possui mais linhas que colunas. Isto acontece porque, caso o ponto campo esteja localizado em uma superfície de interface, ele contribui somente na matriz $\mathbf{H}$. Para melhor esclarecer este fato, reescreve-se a igualdade 3.47 diferenciando-se os contornos que pertencem às superfícies livres dos que pertencem aos contatos:

$$
\left[\begin{array}{ll}
H_{l l} & H_{l c} \\
H_{c l} & H_{c c}
\end{array}\right]\left\{\begin{array}{l}
u_{l} \\
u_{c}
\end{array}\right\}=\left[\begin{array}{c}
G_{l l} \\
G_{c l}
\end{array}\right]\left\{p_{l}\right\}
$$

O índice subscrito $l$ indica que o ponto pertence a uma superfície livre de qualquer subregião, enquanto o índice $c$ indica que pertence ao contato entre duas subregiões quaisquer. Para resolver este sistema falta separar os valores conhecidos dos desconhecidos, 
assim como foi feito na Seção 2.5.3. O resultado é o seguinte sistema de equações:

$$
\mathrm{Ax}=\mathbf{f}
$$

É interessante que se faça uma análise do número de incógnitas deste sistema, assim como foi feito na Seção 3.2 . Sendo $n_{1}$ a soma de todos os pontos pertencentes aos contornos externos das duas subregiões e $n_{2}$ a soma dos pontos pertencentes ao contato, o número de incógnitas $n$ do sistema final de equações é:

$$
n=3 n_{1}+3 n_{2}
$$

Comparando as equações 3.8 e 3.50, nota-se que o sistema de equações obtido por esta técnica é menor que o resultante da formulação da Seção 3.2. Por este motivo, considera-se esta técnica alternativa mais eficiente.

Após resolver o sistema 3.49, as únicas incógnitas restantes no problema são as forças nas interfaces. Como os deslocamentos foram determinados para todos os nós, basta escrever o sistema de equações 2.190 para uma das subregiões para determinar as forças em todas as interfaces das quais esta subregião participa. Neste trabalho isto é feito somente caso haja um interesse específico nestas forças, pois em princípio elas não são necessárias para o cálculo de valores em pontos internos.

\subsubsection{Linhas de carga combinadas à técnica alternativa}

Assim como apresentado no Capítulo 2 pretende-se, neste trabalho, considerar linhas de carga aplicadas no interior do meio tridimensional. A presença de tais cargas implica no surgimento de mais um termo na expressão 3.46, que se torna:

$\mathbf{c}^{i} \mathbf{u}^{i}+\sum_{e=1}^{n e}\left[\frac{E_{e}}{E_{1}} \int_{\bar{\Gamma}_{e}} \mathbf{p}^{*} \mathbf{u} d \bar{\Gamma}_{e}\right]+\sum_{c=1}^{n c}\left[\frac{\Delta E_{m n}}{E_{1}} \int_{\Gamma_{m n}} \mathbf{p}^{*} \mathbf{u} d \Gamma_{m n}\right]=\sum_{e=1}^{n e}\left[\int_{\bar{\Gamma}_{e}} \mathbf{u}_{1}^{*} \mathbf{p} d \bar{\Gamma}_{e}\right]+\sum_{e=1}^{n t o t}\left[\int_{\Gamma e} \mathbf{u}_{j}^{*} \mathbf{s} d \Gamma^{e}\right]$

No termo adicional, ntot é o número total de linhas de carga, o vetor s contém o valor da linha de carga em cada ponto, $\Gamma_{e}$ representa a linha $e$ e $\mathbf{u}_{j}^{*}$ é a solução fundamental de deslocamento da região que contém a linha e. Já foi apresentado no Capítulo 2 como são calculadas as integrais deste termo adicional, portanto aqui parte-se diretamente para a 
análise do sistema de equações. O termo adicional de 3.51 altera o sistema de equações 3.48, que se torna:

$$
\left[\begin{array}{ll}
H_{l l} & H_{l c} \\
H_{c l} & H_{c c}
\end{array}\right]\left\{\begin{array}{l}
u_{l} \\
u_{c}
\end{array}\right\}=\left[\begin{array}{c}
G_{l l} \\
G_{c l}
\end{array}\right]\left\{p_{l}\right\}+\left[\begin{array}{c}
M_{l i} \\
M_{c i}
\end{array}\right]\left\{s_{i}\right\}
$$

em que o índice subscrito $i$ indica que o termo foi escrito para um ponto interno. Escrevendo a equação 3.51 também para todos os nós das linhas de carga, obtém-se:

$$
\left[c_{i i}\right]\left\{u_{i}\right\}+\left[\begin{array}{ll}
H_{i l} & H_{i c}
\end{array}\right]\left\{\begin{array}{c}
u_{l} \\
u_{c}
\end{array}\right\}=\left[G_{i l}\right]\left\{p_{l}\right\}+\left[M_{i i}\right]\left\{s_{i}\right\}
$$

em que os coeficientes da matriz $\left[c_{i i}\right]$ são calculados segundo a expressão 3.45 . As expressões 3.52 e 3.53 podem ser combinadas em um único sistema de equações. Conforme descrito em mais detalhes no Capítulo 2, uma única equação é escrita para cada direção dos nós de topo das linhas de carga, e a contribuição da superfície é igualada a zero nestes nós. Por fim:

$$
\left[\begin{array}{ccc}
H_{l l} & H_{l c} & 0 \\
H_{c l} & H_{c c} & 0 \\
H_{i l} & H_{i c} & c_{i i}
\end{array}\right]\left\{\begin{array}{c}
u_{l} \\
u_{c} \\
u_{i}
\end{array}\right\}=\left[\begin{array}{cc}
G_{l l} & M_{l i} \\
G_{c l} & M_{c i} \\
G_{i l} & M_{i i}
\end{array}\right]\left\{\begin{array}{c}
p_{l} \\
s_{i}
\end{array}\right\}
$$

Em 3.54 o número de equações é igual ao número de incógnitas. Portanto, promovendo uma troca de colunas entre as matrizes, todas as incógnitas podem ser isoladas no lado esquerdo da igualdade e um sistema de equações lineares é obtido, cuja solução são todos os valores incógnitos do problema.

\subsection{Exemplos}

Para testar a formulação alternativa de subregiões apresentada neste Capítulo, dois exemplos são analisados. Cada exemplo é dividido em duas partes, alterando apenas a carga aplicada em cada caso. Para comparar resultados, os problemas não homogêneos são inicialmente resolvidos utilizando o programa comercial ANSYS10.0 empregando uma refinada malha com elementos finitos do tipo SOLID45. Esta malha é composta por 64000 elementos e 71001 nós, podendo ser observada na figura 3.3

A figura apresenta três vistas da mesma malha. Na figura 3.3 a uma vista tridimen- 


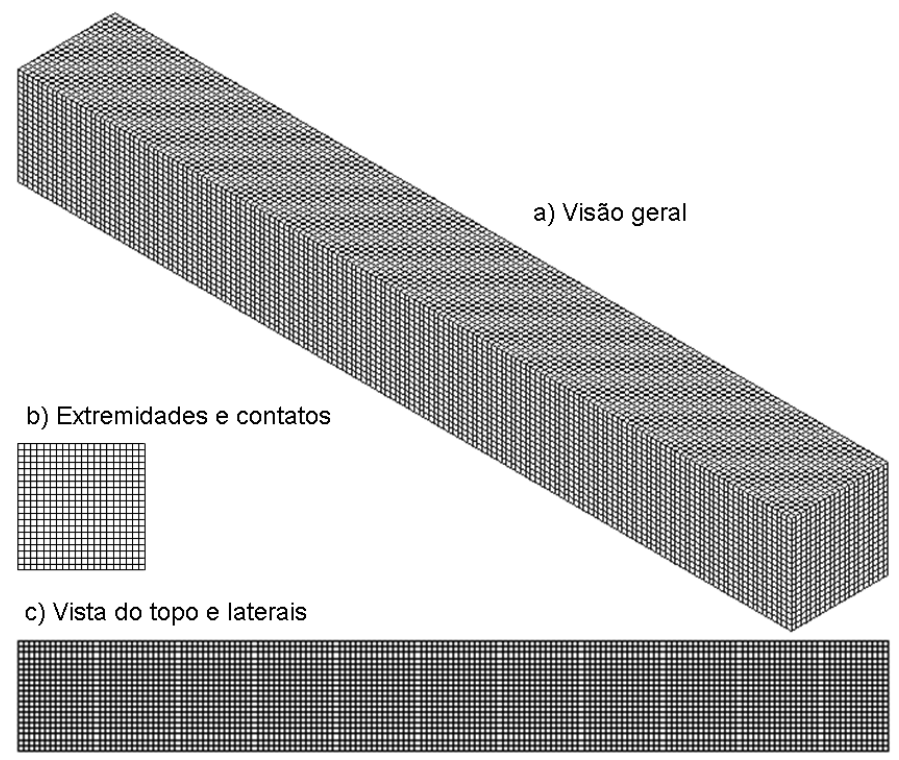

Figura 3.3: Malha de elementos finitos empregada

sional é apresentada, $3.3 \mathrm{~b}$ contém um detalhe da malha empregada nas extremidades e 3.3. ilustra as vistas superior e laterais. Para simular os problemas com o MEC, dois tipos de formulações são empregadas. A primeira é a formulação clássica de subregiões, tal como apresentada na Seção 3.2, e a segunda é a técnica alternativa apresentada na Seção 3.3 .

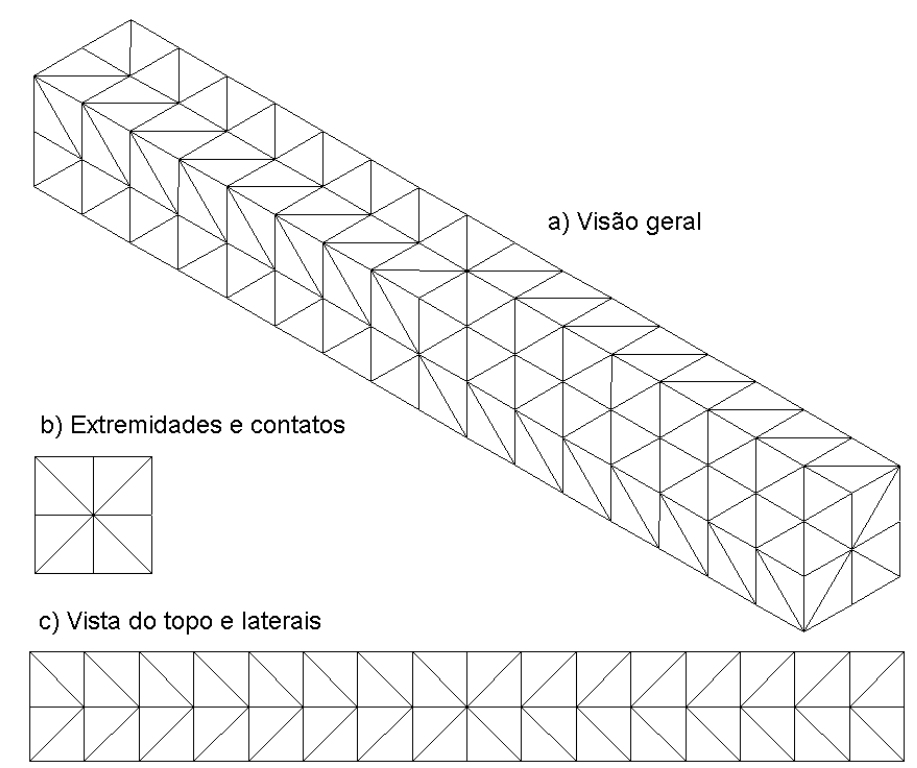

Figura 3.4: Primeira malha de elementos de contorno empregada

Para comparar as duas técnicas do MEC, três malhas diferentes são utilizadas. A primeira, representada na figura 3.4, é identificada nos exemplos como malha 1. Ela possui 138 nós e 272 elementos, podendo ser considerada pobre. Na figura 3.4 é apresentada 
uma vista tridimensional desta malha, 3.4p ilustra um detalhe das extremidades e 3.4 apresenta as vistas superior e laterais. Para cada contato dos exemplos, deve ser inserida uma malha adicional conforme ilustrado na figura $3.4 \mathrm{~b}$.

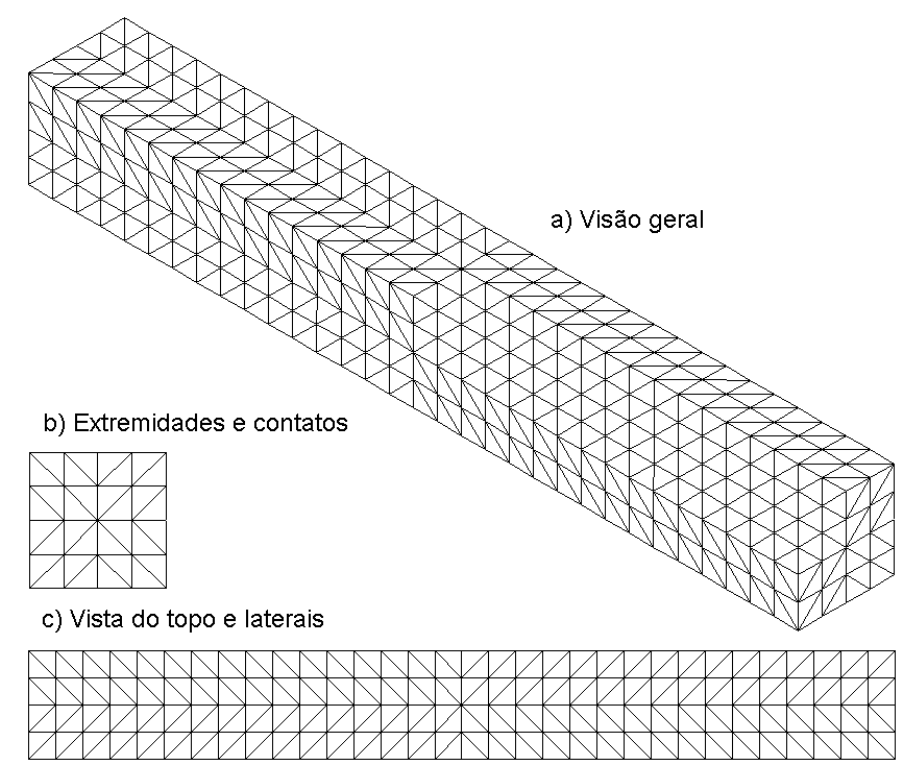

Figura 3.5: Segunda malha de elementos de contorno empregada

A segunda malha, a qual pode ser visualizada na figura 3.5, é identificada nos exemplos como malha 2. Esta é intermediária com 546 nós e 1088 elementos. A figura 3.5é análoga à figura 3.4 . pois as mesmas vistas são apresentadas. Cada interface considedada nos exemplos é modelada com a malha da figura $3.5 \mathrm{~b}$.

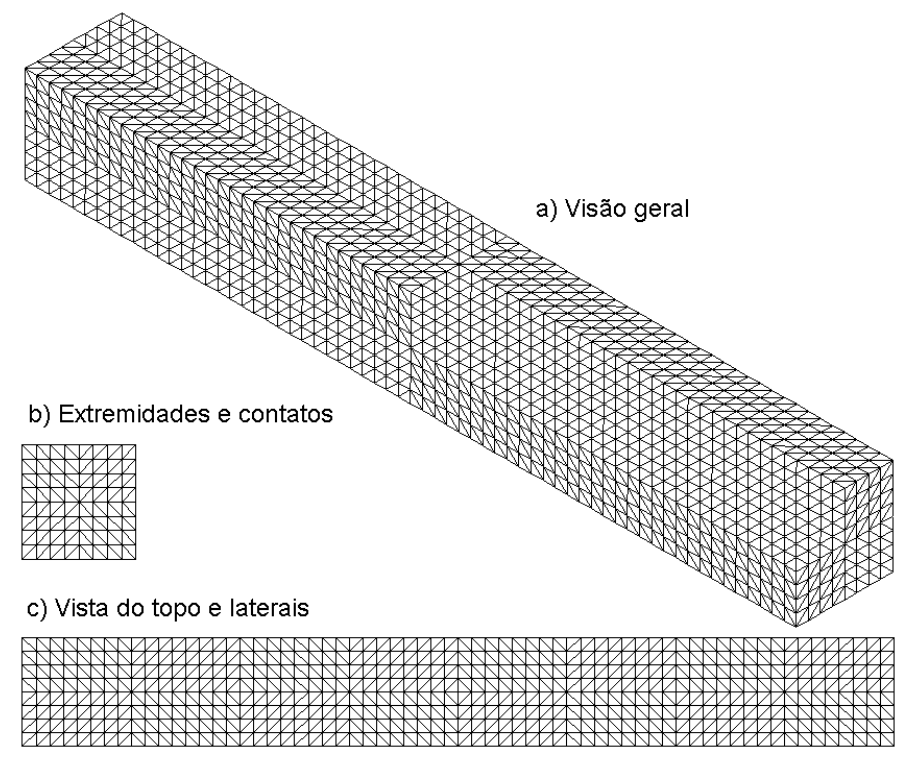

Figura 3.6: Terceira malha de elementos de contorno empregada

A terceira e última malha é identificada nos exemplos como malha 3, e é representada 


\subsection{EXEMPLOS}

na figura 3.6 com as mesmas vistas das figuras $3.4 \mathrm{e} 3.5$. Esta malha pode ser considerada refinada, com 2178 nós e 4352 elementos. Os contatos entre materiais são discretizados com a malha da figura $3.6 \mathrm{~b}$.

\subsubsection{Sólido composto por dois materiais}

Um sólido composto por dois materiais é considerado nesta análise. A primeira parte do problema a ser estudada é apresentada na figura 3.7 .

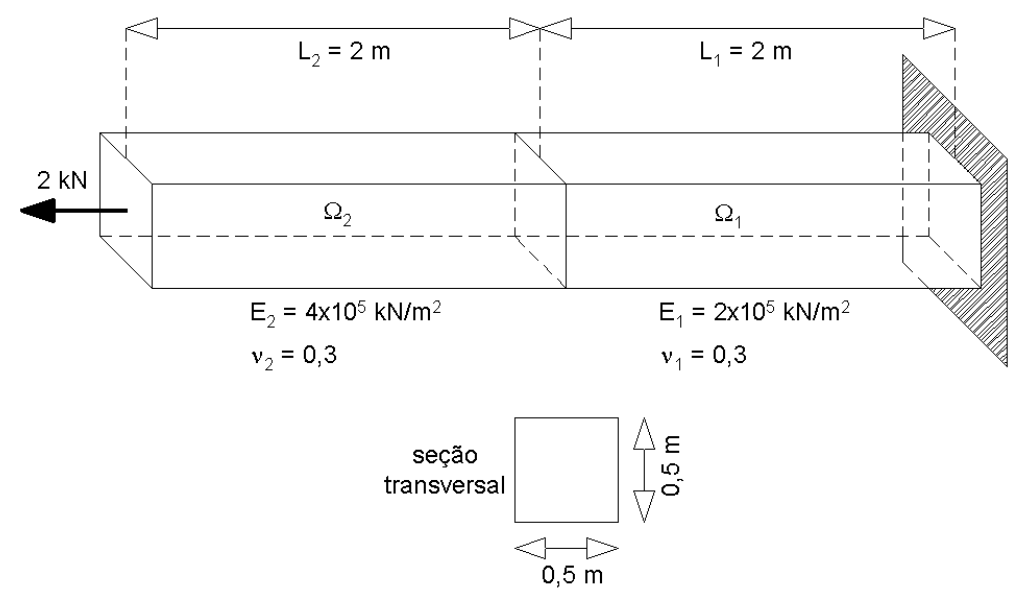

Figura 3.7: Sólido composto por dois materiais

O domínio $\Omega_{1}$ tem um módulo de elasticidade igual a $2 \times 10^{5} \mathrm{kN} / \mathrm{m}^{2}$ e um coeficiente de Poisson de 0,3. O mesmo coeficiente de Poisson é adotado para o domínio $\Omega_{2}$ e um módulo de elasticidade de $4 \times 10^{5} \mathrm{kN} / \mathrm{m}^{2}$. A interface entre os dois materiais é considerada perfeitamente adesiva. Os deslocamentos são restringidos no lado direito do sólido e uma força resultante axial de $2 k N$ é aplicada à outra extremidade. Esta força é uniformemente distribuída na seção transversal da extremidade. A dimensão longitudinal de cada domínio é de $2 m$ e uma seção transversal constante e quadrada com $0,5 m$ de aresta é considerada. O valor que é utilizado para comparar as diferentes formulações é o deslocamento na direção da força, considerando o nó central da seção transversal da extremidade livre.

Por sua simplicidade, é interessante formular este problema analiticamente antes de simulá-lo numericamente. A expressão analítica a ser empregada neste estudo é:

$$
d=\frac{N L}{E A}
$$


em que $d$ é o deslocamento na extremidade livre na direção da carga, $N$ é a força normal, $E$ é o módulo de elasticidade e $A$ é a área da seção transversal. Após aplicar esta expressão separadamente para as duas regiões da figura 3.7, o deslocamento total na extremidade livre é obtido pela soma dos deslocamentos calculados para cada região. Desta forma:

$$
d_{t}=\frac{N L_{1}}{E_{1} A}+\frac{N L_{2}}{E_{2} A}
$$

Substituindo os valores dados na figura 3.7 na expressão 3.56, foi obtido um deslocamento de $1,2000 \times 10^{-4} \mathrm{~m}$. Este valor é utilizado como referência no cálculo dos erros das diferentes formulações numéricas empregadas para simular este problema. Todos os resultados obtidos na análise são organizados na tabela 3.2 .

\begin{tabular}{|c|c|c|}
\hline Técnica numérica & Deslocamento $(\mathrm{m})$ & Erro $(\%)$ \\
\hline \hline ANSY S10.0 & $1,2037 \times 10^{-4}$ & 0,3 \\
\hline \hline Clássica malha 1 & $1,1787 \times 10^{-4}$ & 1,8 \\
\hline Clássica malha 2 & $1,1867 \times 10^{-4}$ & 1,1 \\
\hline Clássica malha 3 & $1,1906 \times 10^{-4}$ & 0,8 \\
\hline \hline Alternativa malha 1 & $1,1816 \times 10^{-4}$ & 1,5 \\
\hline Alternativa malha 2 & $1,1885 \times 10^{-4}$ & 1,0 \\
\hline Alternativa malha 3 & $1,1915 \times 10^{-4}$ & 0,7 \\
\hline
\end{tabular}

Tabela 3.2: Sólido com dois materiais e carga axial

A segunda linha da tabela contém o deslocamento obtido empregando o programa comercial ANSYS10.0 com a malha apresentada na figura 3.3. As três linhas seguintes apresentam os resultados da formulação clássica e as três últimas linhas contém os valores obtidos empregando a técnica alternativa apresentada neste trabalho. Para as duas técnicas de subregiões do MEC três malhas diferentes foram empregadas, as quais podem ser visualizadas nas figuras $3.4,3.5$ e 3.6 .

Todos os erros calculados nas simulações numéricas podem ser considerados pequenos para este exemplo. Este resultado era esperado, pois o comportamento físico deste problema é simples. Entretanto, observa-se que a técnica alternativa foi mais precisa que a clássica comparando os erros calculadao para cada malha.

Para estudar um problema mais complexo, na segunda parte deste exemplo a carga axial é substituída por uma carga transversal. A nova análise a ser feita é ilustrada na figura 3.8 . 


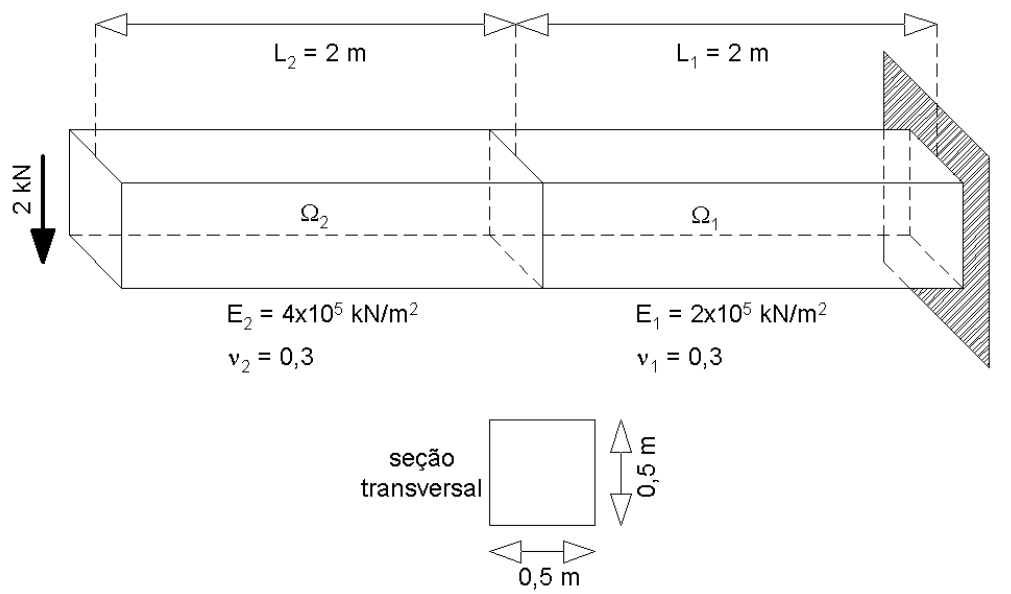

Figura 3.8: Sólido fletido com dois materiais

A carga transversal resultante de $2 k N$ é uniformemente distribuída na seção transversal da extremidade, e o deslocamento é calculado para seu nó central na direção da carga. Para este problema foi gerada a tabela 3.3, a qual é análoga à tabela 3.2. Neste caso, o valor utilizado como referência para o cálculo dos erros foi de $3,8395 \times 10^{-4} \mathrm{~m}$, obtido com o programa comercial $A N S Y S 10.0$ e a malha da figura 3.3 .

\begin{tabular}{|c|c|c|}
\hline Técnica numérica & Deslocamento $(\mathrm{m})$ & Erro $(\%)$ \\
\hline \hline Clássica malha 1 & $5,5499 \times 10^{-2}$ & 44,5 \\
\hline Clássica malha 2 & $4,4940 \times 10^{-2}$ & 17,0 \\
\hline Clássica malha 3 & $4,0377 \times 10^{-2}$ & 5,2 \\
\hline \hline Alternativa malha 1 & $4,0621 \times 10^{-2}$ & 5,8 \\
\hline Alternativa malha 2 & $4,0585 \times 10^{-2}$ & 5,7 \\
\hline Alternativa malha 3 & $3,9289 \times 10^{-2}$ & 2,3 \\
\hline
\end{tabular}

Tabela 3.3: Sólido com dois materiais e carga transversal

Pode ser observado que, para este caso, mesmo com a malha 3 , o resultado obtido com a formulação clássica não foi satisfatório. Por outro lado, o deslocamento calculado com a técnica alternativa foi mais preciso. É importante notar que o erro da técnica alternativa empregando a malha 1 foi próximo ao da formulação clássica com a malha 3. Isto significa que, para este exemplo, a formulação clássica precisou de uma malha com 2178 nós para superar a precisão obtida com a técnica alternativa empregando uma malha com apenas 138 nós. 


\subsubsection{Sólido composto por quatro materiais}

O objetivo deste segundo exemplo é analizar um sólido composto por quatro materiais. O problema a ser considerado é apresentado na figura 3.9 .

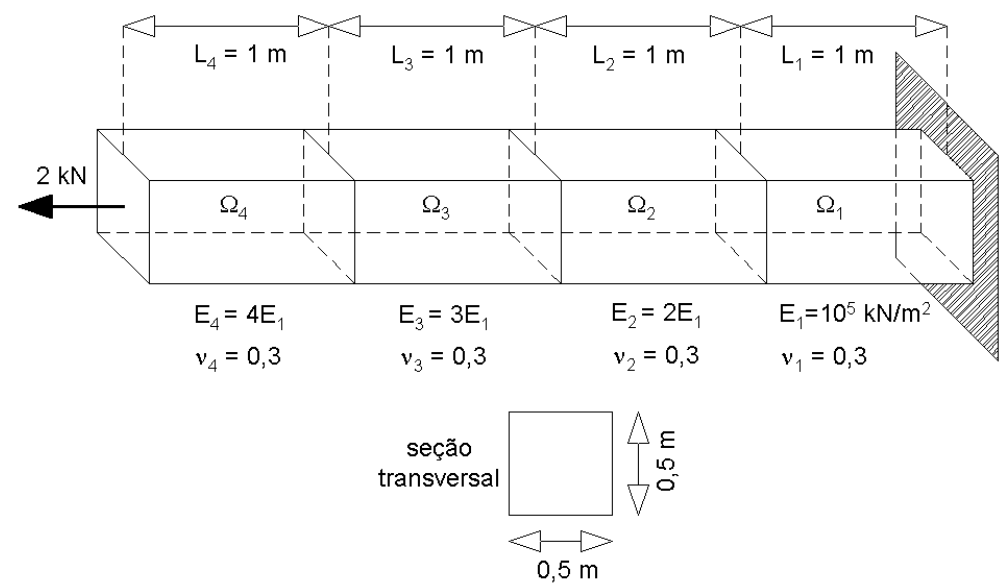

Figura 3.9: Sólido composto por quatro materiais

O domínio $\Omega_{1}$ possui módulo de elasticidade igual a $10^{5} \mathrm{kN} / \mathrm{m}^{2}$ e coeficiente de Poisson de 0,3. O mesmo coeficiente de Poisson é adotado para os demais domínios, entretanto seus módulos de elasticidade são diferentes. No domínio $\Omega_{2}$ o módulo de elasticidade é $2 E_{1}$, em $\Omega_{3}$ é $3 E_{1}$ e em $\Omega_{4}$ é $4 E_{1}$. Todas as interfaces são consideradas perfeitamente adesivas. Os deslocamentos são restringidos no lado direito do sólido e uma força resultante axial de $2 k N$ é aplicada na outra extremidade. Esta força é considerada uniformemente distribuída na seção transversal da extremidade. A dimensão longitudinal de cada domínio é de $1 \mathrm{~m}$ e é considerada uma seção transversal quadrada constante com aresta de $0,5 \mathrm{~m}$. O valor utilizado para comparar as diferentes formulações é o deslocamento na direção da força, considerando o nó central da seção transversal da extremidade.

Assim como feito no exemplo anterior, é possível analisar este problema analiticamente. Assim, aplicando a expressão 3.55 separadamente para cada região da figura 3.9, o deslocamento total na extremidade livre pode ser calculado pela soma dos deslocamentos calculados para cada região. Isto é:

$$
d_{t}=\frac{N L_{1}}{E_{1} A}+\frac{N L_{2}}{E_{2} A}+\frac{N L_{3}}{E_{3} A}+\frac{N L_{4}}{E_{4} A}
$$

Substituindo em 3.57 os valores dados na figura 3.9, é obtido um deslocamento de $1,6667 \times 10^{-4} \mathrm{~m}$. Simulando este problema com as mesmas técnicas numéricas empregadas 
no exemplo anterior, obtém-se os valores da tabela 3.4 .

\begin{tabular}{|c|c|c|}
\hline Técnica numérica & Deslocamento $(\mathrm{m})$ & Erro $(\%)$ \\
\hline \hline ANSYS10.0 & $1,6631 \times 10^{-4}$ & 0,2 \\
\hline \hline Clássica malha 1 & $1,6193 \times 10^{-4}$ & 2,8 \\
\hline Clássica malha 2 & $1,6375 \times 10^{-4}$ & 1,8 \\
\hline Clássica malha 3 & $1,6466 \times 10^{-4}$ & 1,2 \\
\hline \hline Alternativa malha 1 & $1,6286 \times 10^{-4}$ & 2,3 \\
\hline Alternativa malha 2 & $1,6430 \times 10^{-4}$ & 1,4 \\
\hline Alternativa malha 3 & $1,6492 \times 10^{-4}$ & 1,0 \\
\hline
\end{tabular}

Tabela 3.4: Sólido com quatro materiais e carga axial

Os resultados são organizados da mesma forma que na tabela 3.2 , e empregando as mesmas malhas. Apesar dos maiores erros calculados para as simulações com o MEC, quando comparados ao obtido na análise com o MEF, todas as simulações podem ser consideradas precisas. Este resultado era novamente esperado devido à simplicidade do problema analisado. No entanto, é importante notar que, considerando as mesmas malhas, a técnica alternativa foi superior à clássica.

Na parte final deste segundo exemplo, assim como feito no exemplo anterior, a carga axial é substituída por uma transversal. O novo problema a ser modelado é ilustrado na figura 3.10 .

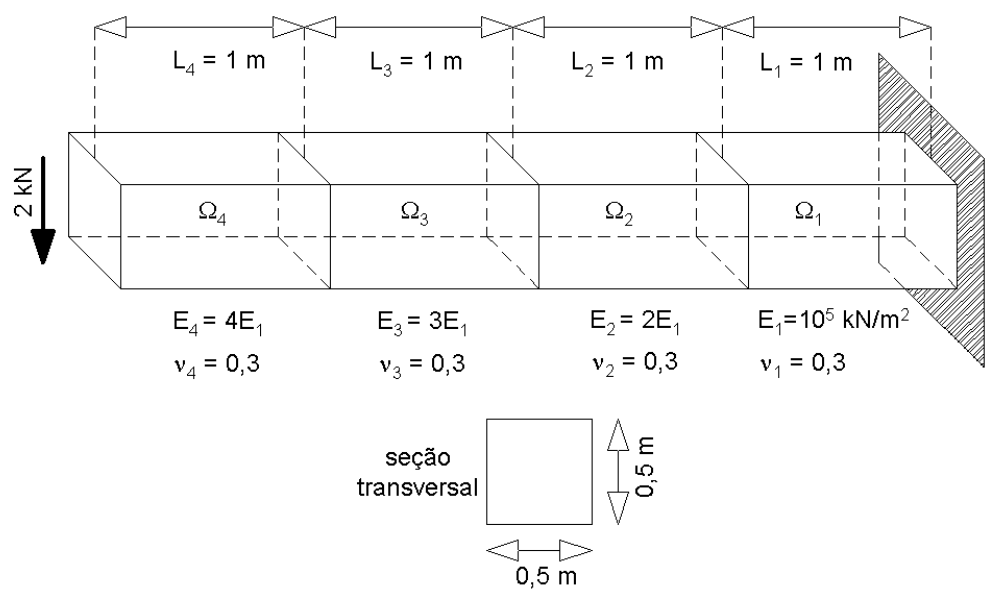

Figura 3.10: Sólido fletido com quatro materiais

O deslocamento é calculado para o nó central da seção transversal da extremidade, na qual a carga resultante é uniformemente distribuída, e considerando a direção da carga. Os resultados obtidos nas simulações numéricas são organizados na tabela 3.5, sendo análoga 
às anteriormente apresentadas. O valor obtido no $A N S Y S 10.0$, de $6,2550 \times 10^{-2} \mathrm{~m}$, é utilizado como referência no cálculo dos erros.

\begin{tabular}{|c|c|c|}
\hline Técnica numérica & Deslocamento $(\mathrm{m})$ & Erro $(\%)$ \\
\hline \hline Clássica malha 1 & $1,1490 \times 10^{-1}$ & 83,7 \\
\hline Clássica malha 2 & $8,0267 \times 10^{-2}$ & 28,3 \\
\hline Clássica malha 3 & $6,7629 \times 10^{-2}$ & 8,1 \\
\hline \hline Alternativa malha 1 & $6,7883 \times 10^{-2}$ & 8,5 \\
\hline Alternativa malha 2 & $6,6781 \times 10^{-2}$ & 6,8 \\
\hline Alternativa malha 3 & $6,4193 \times 10^{-2}$ & 2,6 \\
\hline
\end{tabular}

Tabela 3.5: Sólido com quatro materiais e carga transversal

A diferença nos resultados obtidos nas simulações com o MEC são ainda mais significativos que no exemplo anterior. O erro com a formulação clássica se tornou demasiadamente elevado, enquanto que o erro da técnica alternativa empregando a malha 3 pode ainda ser considerado aceitável. Mais uma vez, a precisão da técnica alternativa empregando a malha 1 foi próximo do erro da formulação clássica com a malha 3. Considerando estes testes pode-se concluir que a técnica alternativa é, de fato, mais precisa que a clássica.

Para avaliar o erro introduzido pela consideração de um mesmo coeficiente de Poisson para todas as regiões, esta última análise foi novamente simulada no ANSYS10.0 adotando diferentes coeficientes de Poisson para cada região. Desta forma, para a região $\Omega_{1}$ um coeficiente de Poisson de 0,15 foi adotado, para $\Omega_{2}$ um coeficiente de Poisson de $0,25,0,35$ para a região $\Omega_{3}$ e 0,45 para $\Omega_{4}$. Um deslocamento de $6,3137 \times 10^{-2} m$ foi calculado para o mesmo ponto considerado anteriormente, resultando em um erro de apenas 0,9 \% em relação ao valor previamente obtido. Este erro pode ser considerado irrelevante quando comparado à imprecisão apresentada pela formulação clássica nos exemplos.

\subsection{Considerações finais}

Foram apresentadas duas formulações neste Capítulo para a análise de sólidos em contato. A primeira aplica condições de equilíbrio de forças e compatibilidade de deslocamentos para montar, a partir das equações de cada sólido, um sistema de equações válido para todas as subregiões. Esta técnica é mais conhecida na literatura e suas principais desvantagens são a obtenção de um sistema global com mais equações e incógnitas, resultando em um maior tempo de processamento, e uma perda de precisão devido às 
aproximações adotadas nos contatos entre diferentes regiões.

A segunda formulação monta o sistema global de forma mais direta, pois integra, para cada ponto fonte, todos os contornos definidos no problema. A estratégia é relacionar as soluções fundamentais de cada subregião uma com a outra segundo seu módulo de elasticidade. O sistema de equações resulta menor quando comparado ao da primeira técnica, implicando em um menor tempo de processamento. Além disso os resultados obtidos são mais precisos, pois não é necessário aproximar as tensões de contato entre diferentes regiões.

A desvantagem da formulação alternativa é que ela só é válida para problemas nos quais todas as subregiões têm mesmo coeficiente de Poisson, no entanto esta restrição pode ser considerada pouco relevante para as aplicações pretendidas neste trabalho. A técnica alternativa é empregada, nos próximos Capítulos, para simular maciços de solos, aproximando-os de forma semelhante à utilizada no trabalho de Gibson (1967). Desta forma o coeficiente de Poisson é considerado constante em todo o maciço, mas o módulo de elasticidade pode ser variável com a profundidade.

Além disto foram feitos, ao final do último exemplo, testes com esta formulação considerando problemas com diferentes coeficientes de Poisson e concluiu-se que o erro introduzido pela consideração de um coeficiente de Poisson médio é pouco relevante para o cálculo dos deslocamentos. Desta forma, torna-se viável empregar esta formulação em problemas mais gerais. 


\section{Capítulo 4}

\section{Estudos sobre elementos infinitos}

\subsection{Introdução}

O objetivo principal deste Capítulo é apresentar alguns estudos feitos na área de elementos infinitos, aplicando sua teoria no desenvolvimento de elementos de contorno infinitos. A análise é voltada para problemas estáticos, considerando sólidos homogêneos, contínuos e elástico-linerares. São empregados elementos de contorno infinitos em conjunto com elementos de contorno convencionais no programa desenvolvido neste trabalho, tornando possível a simulação do solo como um sólido infinito nas direções radiais. Para maiores esclarecimentos acerca da teoria dos elementos infinitos, recomenda-se consultar Bettess (1992). Este livro foi utilizado como base para a redação de parte deste Capítulo.

Na prática, é difícil encontrar um problema de engenharia que envolva um domínio de fato infinito. Desta forma, poderia ser considerado desnecessário o estudo de modelos matemáticos para a simulação de tais domínios. Entretanto, existem muitos casos em que, embora o domínio seja finito, seu contorno é tão distante que não pode ser claramente definido ou a região de interesse não é precisamente delimitada. Considera-se, por exemplo, uma edificação qualquer apoiada sobre o solo e sujeita a algum tipo de carga. Sabe-se que o solo não é infinito, ele possui um contorno localizado a uma certa distância. Entretanto, é intuitivo que, neste caso, a distância exata do contorno bem como uma definição detalhada de sua geometria não é importante, tendo pouca influência nos resultados. Desta forma, torna-se mais prático analisar este problema considerando-se o solo como um domínio infinito.

A forma mais usual de modelar o solo em problemas de interação solo-estrutura, empre- 
gando métodos numéricos tais como o método dos elementos finitos (MEF) ou o método dos elementos de contorno (MEC), é criar uma malha de elementos extensa o suficiente para que a contribuição dos elementos localizados em seus limites seja insignificante. Considerando mais especificamente modelos que simulam o solo como um sólido tridimensional empregando o MEC, no contexto deste tabalho, torna-se necessária a criação de uma extensa malha de elementos de contorno planos. Esta malha deve estender-se até uma certa distância da estrutura, de forma que a contribuição dos elementos mais distantes seja muito pequena quando comparada à contribuição dos elementos próximos à estrutura. A imposição desta condição torna o número de elementos da malha elevado, prejudicando o processamento e muitas vezes até inviabilizando a simulação do problema.

Em vista destes fatores, a solução proposta neste trabalho é o desenvolvimento de um elemento de contorno cuja formulação seja semelhante ao do elemento convencional, mas que possua características que permitam simular domínios que se extendem até o infinito. Uma forma de conseguir estas características é empregar alguns conceitos utilizados nos elementos infinitos (EIs), aplicando-os para tornar infinito o elemento de contorno convencional. Desta forma, antes de abordar elementos de contorno infinitos (ECIs), é necessário estudar e compreender parte da teoria dos EIs.

Assim, na Seção 4.2 é feita uma abordagem geral dos EIs, apresentando seus principais conceitos. Na Seção 4.3 é apresentada a formulação do EI com funções de decaimento. Na sequência descreve-se, na Seção 4.4. o EI mapeado de Zienkiewicz. Os conceitos são então empregados na Seção 4.5 para o desenvolvimento do ECI empregado neste trabalho. A Seção 4.6 contém exemplos de aplicação da formulação desenvolvida e por fim, na Seção 4.7. são feitas as considerações finais do Capítulo.

\subsection{Conceitos iniciais}

O primeiro elemento infinito foi desenvolvido no trabalho de Ungless (1973). A idéia básica deste elemento foi utilizar uma função de forma cuja variação nas direções radiais fosse do tipo $1 /(1+r)$, em que $r$ é a distância radial. O elemento deste trabalho é tridimensional e possui uma base triangular, definida no plano local $x_{1} x_{2}$. Sua forma é próxima a de um prisma, sendo sua dimensão na direção $x_{3}$ infinita. As paredes do elemento na direção $x_{3}$, se prolongadas para além da base, convergem para um ponto 
chamado de "pólo".

Ungless realizou testes considerando uma carga unitária aplicada na superfície de um semi-espaço infinito, comparando os resultados com soluções analíticas. Houve boa concordância nos valores, tanto para cargas perpendiculares à superficie como para cargas tangentes à ela.

Outros tipos de elementos infinitos foram criados após o trabalho de Ungless. Em Bettess (1977) é descrita uma forma de obter elementos infinitos a partir de qualquer elemento finito convencional. Em linhas gerais, o procedimento consiste em multiplicar a função de forma do elemento original por uma função de decaimento. Esta função deve ser escolhida de forma apropriada, de acordo com o tipo de problema a ser analisado.
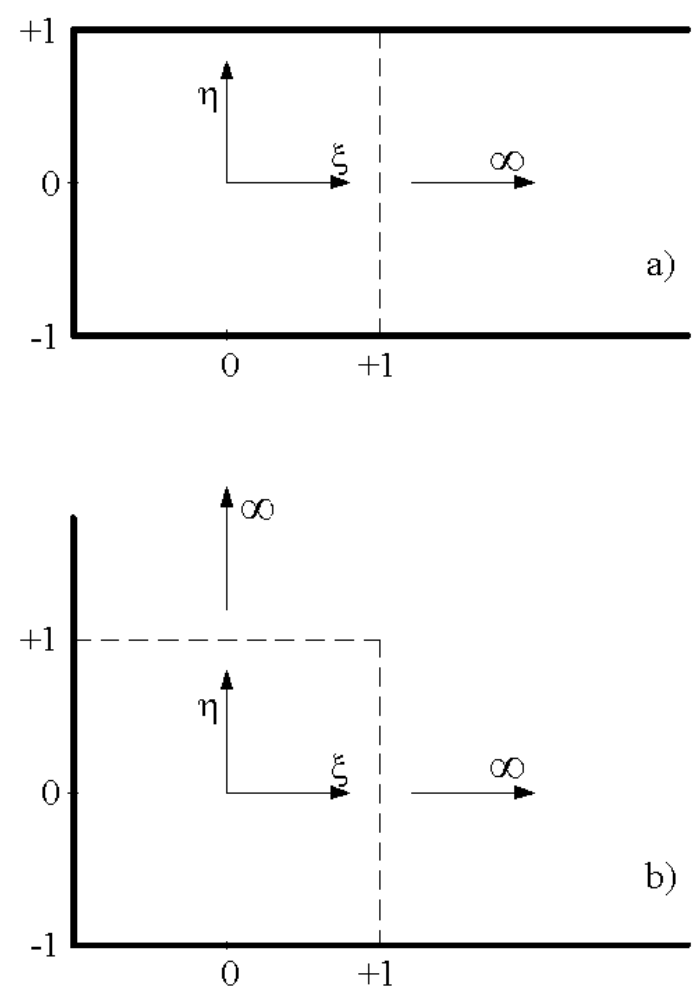

Figura 4.1: Exemplos de elementos infinitos bidimensionais com funções de decaimento

Na figura 4.1 são ilustrados exemplos deste tipo de elemento. O elemento $a$ é infinito somente na direção adimensional $\xi$, enquanto que $b$ é infinito tanto em $\xi$ como em $\eta$. A função de forma é multiplicada pela função de decaimento somente nas direções que se extendem ao infinito. Portanto, no caso $a$, apenas a direção $\xi$ tem decaimento, enquanto que ambas as direções têm decaimento em $b$.

As primeiras funções de decaimento utilizadas por Betess foram as exponenciais. Isto possibilitou que as integrais sobre os elementos fossem calculadas analiticamente. Os 
primeiros problemas analisados por Betess empregando elementos infinitos foram unidimensionais, passando aos bidimensionais e finalmente aos tridimensionais.

Os elementos infinitos seguintes aos de Bettess (1977) divergiram em sua formulação para duas linhas principais. A primeira utiliza funções de decaimento, tal como Bettess (1977), para transformar um elemento finito qualquer em infinito. A teoria referente a estes elementos é descrita com mais detalhes na Seção 4.3. Na segunda linha o elemento infinito também é obtido a partir de um elemento finito qualquer, mas não a partir de funções de decaimento. Neste caso o domínio do elemento finito é mapeado em outro domínio, o qual tem dimensões infinitas. Estes elementos são denominados, neste trabalho, de elementos infinitos mapeados, e a partir da Seção 4.4 é feito um estudo mais detalhado de suas características.

\subsection{Elementos infinitos com funções de decaimento}

Este tipo de elemento infinito pode ser obtido a partir de um elemento finito qualquer. Suas funções de forma permanecem inalteradas, sendo apenas multiplicadas por funções de decaimento. Estas funções de decaimento devem ser escolhidas de forma a assegurar que o comportamento da resposta do elemento no infinito seja coerente com o problema físico que se deseja representar. Isto significa que a variável em questão deve tender, em grandes distâncias, a um valor específico.

Considera-se um elemento finito qualquer com funções de forma $P_{i}(\xi, \eta)$, em que $\xi$ e $\eta$ são coordenadas locais e $i$ é a numeração local dos nós. Adotando funções de decaimento $f_{i}(\xi, \eta)$, as novas funções de forma podem ser obtidas pela seguinte expressão:

$$
N_{i}(\xi, \eta)=f_{i}(\xi, \eta) P_{i}(\xi, \eta)
$$

sendo que em 4.1 não há soma no índice $i$. Uma característica fundamental das funções de decaimento é que elas devem ter valor unitário em seus nós correspondentes. Isto ocorre porque no próprio nó a distância é igual a zero, não havendo decaimento algum a ser considerado. Assim:

$$
f_{i}\left(\xi_{i}, \eta_{i}\right)=1
$$

Em outros nós não há exigência alguma em relação à função de decaimento desde que 
$N_{i}(\xi, \eta)$ tenda, em grandes distâncias, a um valor específico. Qualquer que seja a função $f$ escolhida, as derivadas de $N$ podem ser calculadas pela regra da cadeia. Considerando decaimento somente na direção $\xi$ :

$$
\begin{gathered}
\frac{\partial N_{i}}{\partial \xi}=\frac{\partial f_{i}}{\partial \xi} P_{i}+f_{i} \frac{\partial P_{i}}{\partial \xi} \\
\frac{\partial N_{i}}{\partial \eta}=f_{i} \frac{\partial P_{i}}{\partial \eta}
\end{gathered}
$$

e considerando decaimento em ambas as direções:

$$
\begin{gathered}
\frac{\partial N_{i}}{\partial \xi}=\frac{\partial f_{i}}{\partial \xi} P_{i}+f_{i} \frac{\partial P_{i}}{\partial \xi} \\
\frac{\partial N_{i}}{\partial \eta}=\frac{\partial f_{i}}{\partial \eta} P_{i}+f_{i} \frac{\partial P_{i}}{\partial \eta}
\end{gathered}
$$

Também não há, nas expressões 4.3, 4.4, 4.5 e 4.6, soma no índice $i$. Caso fosse necessária, a segunda derivada também poderia ser encontrada aplicando novamente a regra da cadeia em cada parcela das equações.

\subsubsection{Funções de decaimento exponenciais}

Uma possível função de decaimento poderia ser do tipo $e^{-x}$. Para uma melhor visualização das expressões, ao longo deste texto será empregada a seguinte notação:

$$
e^{-x}=\exp (-x)
$$

com

$$
e=2,71828183 \ldots
$$

As funções de decaimento do tipo $\exp (-x)$ foram as primeiras a serem empregadas na literatura. Elas têm a vantagem de tender a zero mais rapidamente que qualquer polinômio, dominando a convergência na medida em que $x$ se torna muito grande e portanto garantindo que o valor da variável em questão tenda a zero no infinito. Também são matematicamente fáceis de serem manipuladas. Uma forma mais precisa para uma 
função de decaimento deste tipo é:

$$
f_{i}(\xi, \eta)=\exp \left[\frac{\left(\xi_{i}-\xi\right)}{L}\right]
$$

A expressão 4.9 é válida para decaimento somente na direção positiva de $\xi$, e sua severidade ao longo da distância entre os nós é regulada pelo comprimento $L$. No nó $i$ o valor de $\xi$ é $\xi_{i}$, zerando o expoente e garantindo que a exigência expressa na equação 4.2 seja satisfeita. Caso haja decaimento nas duas direções, $\xi$ e $\eta$, a expressão se torna:

$$
f_{i}(\xi, \eta)=\exp \left[\frac{\left(\xi_{i}+\eta_{i}-\xi-\eta\right)}{L}\right]
$$

Para que o decaimento ocorra na direção negativa de $\xi$, deve ser feita uma alteração trivial na expressão 4.9 .

$$
f_{i}(\xi, \eta)=\exp \left[\frac{\left(\xi-\xi_{i}\right)}{L}\right]
$$

É possível estender a formulação para considerar três direções de decaimento. No caso das expressões 4.9, 4.10 e 4.11 foram considerados elementos bidimensionais, com decaimento nas direções dos eixos locais $\xi$ e $\eta$.

\subsubsection{Funções de decaimento recíprocas}

Para o caso tridimensional a função de Green para problemas potenciais é do tipo $L / r$, em que $r$ é o raio e $L$ é um comprimento típico do problema. Buscar um elemento infinito cujo comportamento seja essencialmente da forma $1 / r$ é natural, uma vez que esta função descreve grande parte dos problemas reais. Para que um elemento infinito com funções de decaimento tenha tal comportamento, é necessário que suas funções de forma finais $N_{i}(\xi, \eta)$, dadas na expressão 4.1, sejam do tipo $1 / r$. A seguir, é demonstrado um procedimento simples para escolher funções de decaimento de maneira que $N_{i}(\xi, \eta)$ seja deste tipo.

Toma-se inicialmente uma fução de decaimento da forma:

$$
f_{i}(\xi)=\left(\frac{\xi_{i}-\xi_{0}}{\xi-\xi_{0}}\right)^{n}
$$

em que $\xi_{0}$ é algum ponto de origem. Este ponto deve estar do lado de fora do elemento in- 
finito, ou seja, no lado oposto ao que o elemento vai se estender ao infinito. Normalmente, caso o decaimento ocorra na direção positiva de $\xi$, então $\xi_{0}<-1$. Isto evita possíveis singularidades.

O valor a ser escolhido para $n$ depende das funções de forma originais do elemento, $P_{i}$. Deve-se adotar um $n$ superior à maior potência de $\xi$ que estará presente nas funções de forma finais $N_{i}$. Isto garante que, a medida que $\xi$ tenda ao infinito, $N_{i}$ tenda a $1 / \xi$, conforme justificado anteriormente. Mesmo com esta regra podem surgir dificuldades para a escolha de $n$, uma vez que mais de um valor pode ser possível. Neste caso, um $n$ muito pequeno pode prejudicar os resultados de $N_{i}$ em grandes distâncias. Por outro lado, um $n$ muito grande pode resultar na omissão de importantes características do problema físico analisado. Neste caso, uma opção para chegar ao valor ideal é realizar experimentos variando $n$, testando inclusive valores não inteiros.

Para decaimento na direção de $\xi$ negativo, a expressão inicial 4.12 se torna:

$$
f_{i}(\xi)=\left(\frac{\xi_{0}-\xi_{i}}{\xi-\xi_{0}}\right)^{n}
$$

Neste caso, $\xi_{0}$ deve ser maior que 1 para evitar as singularidades. A função de decaimento inicial pode também ser generalizada para duas dimensões, ou seja:

$$
f_{i}(\xi, \eta)=\left(\frac{\xi_{i}-\xi_{0}}{\xi-\xi_{0}}\right)^{n}\left(\frac{\eta_{i}-\eta_{0}}{\eta-\eta_{0}}\right)^{m}
$$

Devem ser então escolhidas duas coordenadas de origem, $\xi_{0}$ e $\eta_{0}$, também devendo ser ambas menores que -1 para evitar singularidades. Os valores de $n$ e $m$ serão dependentes das maiores potências de $\xi$ e $\eta$, respectivamente, nas funções de forma iniciais do elemento finito.

\subsubsection{Integração numérica - Gauss-Legendre}

Para efetuar numericamente as integrais para o cálculo dos termos da matriz de rigidez de um elemento infinito, é possível aplicar a fórmula de Gauss-Legendre. Maiores detalhes a respeito deste método podem ser consultados em Bettess (1992). Os limites de integração da fórmula são -1 e +1 , e as integrais do elemento infinito são geralmente -1 e $\infty$. Tornase então necessário mapear as coordenadas e pesos dos pontos para possibilitar o cálculo de tais integrais empregando-se este método. Existem uma infinidade de maneiras de 
fazer este mapeamento, mas é demonstrada aqui somente a forma empregada por Davis e Rabinowitz (1975). Utiliza-se a seguinte igualdade:

$$
x(t)=\frac{a+b t}{1+t}, \quad \frac{d x}{d t}=\frac{b-a}{(1+t)^{2}}
$$

$\mathrm{Ou}$

$$
t(x)=\frac{a-x}{x-b}, \quad \frac{d t}{d x}=\frac{b-a}{(x-b)^{2}}
$$

Assim, quando $x=a$ a variável $t$ é zero e quando $x=b$ a variável $t$ é $\infty$. Portanto, pode-se escrever a seguinte equação:

$$
\int_{0}^{\infty} f(t) d t=(b-a) \int_{a}^{b} f\left(\frac{a-x}{x-b}\right) \frac{d x}{(x-b)^{2}}
$$

Em 4.17, $f(t)$ é a função original que se pretende integrar. Desta forma, uma integral cujos limites originais são zero e $\infty$ pode ser transformada para ter limites $a$ e $b$ quaisquer. No caso de uma transformação para $a=0$ e $b=1$, por exemplo, escreve-se:

$$
\int_{0}^{\infty} f(t) d t=\int_{0}^{1} f\left(\frac{x}{1-x}\right) \frac{d x}{(1-x)^{2}}
$$

Desta forma, a nova coordenada $t$ do ponto de integração pode ser obtida a partir da coordenata original $x$ a partir da relação:

$$
t=\frac{x}{1-x}
$$

e o novo peso $\bar{w}$ do ponto relaciona-se ao peso original $w$ pela igualdade:

$$
\bar{w}=\frac{w}{(1-x)^{2}}
$$

Para o caso em que os limites de integração iniciais são -1 e $\infty$ é necessária mais uma transformação, que é:

$$
\xi(x)=t(x)-1=\frac{a-x}{x-b}-1, \quad \frac{d \xi}{d x}=\frac{b-a}{(x-b)^{2}}
$$

Assim, quando $x=a$ a variável $\xi=-1$ e quando $x=b$ a variável $\xi=\infty$. Isto permite 
escrever a seguinte relação:

$$
\int_{-1}^{\infty} f(\xi) d \xi=(b-a) \int_{a}^{b} f\left(\frac{a-x}{x-b}-1\right) \frac{d x}{(x-b)^{2}}
$$

Mais especificamente para o caso da integração numérica de Gauss em que os limites de integração são $a=-1$ e $b=1$, a igualdade se torna:

$$
\int_{-1}^{\infty} f(\xi) d \xi=\int_{-1}^{1} f\left(\frac{2 x}{1-x}\right) \frac{2 d x}{(1-x)^{2}}
$$

A nova coordenada $\xi$ do ponto de Gauss pode ser obtida a partir da coordenada $x$ original empregando a seguinte igualdade:

$$
\xi=\frac{2 x}{1-x}
$$

e o novo peso $\hat{w}$ do ponto de Gauss se relaciona com o peso original $w$ da seguinte forma:

$$
\hat{w}=\frac{2 w}{(1-x)^{2}}
$$

\subsection{O elemento infinito mapeado de Zienkiewicz}

A maioria dos elementos infinitos presentes na literatura são baseados em algum tipo de mapeamento. Muitas vezes dois mapeamentos são empregados, um para a função de forma e outro para a integração numérica que, geralmente, é a de Gauss-Legendre. Este procedimento tem a vantagem de manter inalteradas as coordenadas e pesos originais dos pontos de integração. A única modificação a ser feita então no programa do elemento finito original para torná-lo infinito é na parte da montagem da matriz Jacobiana, conforme será demonstrado a seguir.

Considera-se, como ponto de partida, um elemento finito quadrático unidimensional de três nós, conforme ilustrado na figura 4.2. A obtenção do elemento infinito mapeado se baseia no mapeamento da coordenada $x$, cuja origem se encontra do lado esquerdo da figura, para a coordenada homogênea $\xi$ do elemento de origem. Desta forma o elemento infinito resulta também com três nós, cada qual correspondente a um dos nós do elemento de origem.

O primeiro nó, de coordenada $\xi=-1$, deve ser mapeado para a coordenada $x=x_{1}$ 


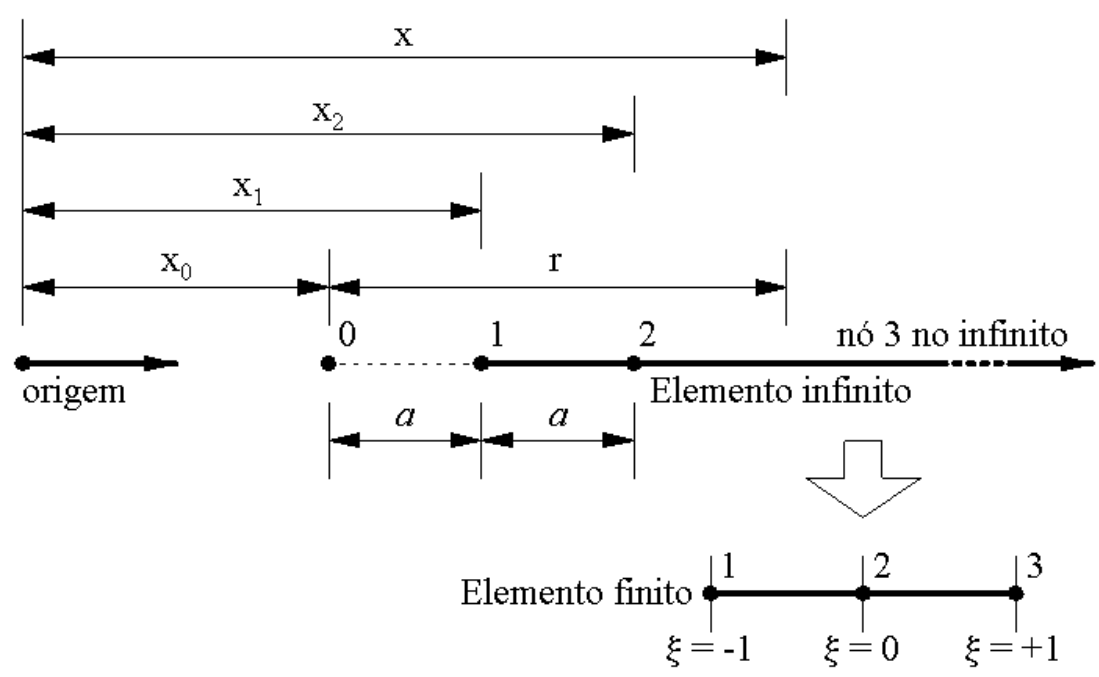

Figura 4.2: O elemento infinito mapeado de Zienkiewicz

do sistema de coordenadas global. O segundo, de coordenada $\xi=0$, deve ser mapeado para $x=x_{2}$. Finalmente, o terceiro nó, que se encontra na coordenada $\xi=+1$, deve ser mapeado para uma distância infinita $x=x_{3}=\infty$. Existe, no domínio $x$, mais um ponto importante além dos três nós do elemento infinito. Este ponto, localizado em $x=x_{0}$, é denominado como "pólo" do elemento, e sua função é servir de referência para as funções de mapeamento. Será visto mais adiante que, quando a coordenada homogênea tende a infinito, a coordenada global tende para a posição do pólo.

Na formulação que será aqui apresentada, a distância do pólo ao nó 1 é igual à distância do nó 1 ao nó 2. Esta distância está indicada na figura pela letra $a$. Também é possível que estas distâncias não sejam iguais, mas este caso não será estudado aqui.

O mapeamento do elemento de origem no elemento infinito pode ser feito a partir de uma função de mapeamento. É necessário que cada ponto do elemento final tenha um único ponto correspondente no elemento inicial. Uma possível opção é:

$$
x=\tilde{N}_{0}(\xi) x_{0}+\tilde{N}_{2}(\xi) x_{2}
$$

em que

$$
\tilde{N}_{0}(\xi)=\frac{-\xi}{1-\xi}, \quad \tilde{N}_{2}(\xi)=1+\frac{\xi}{1-\xi}
$$

Assim, em $\xi=-1$, tem-se:

$$
x=\frac{-(-1)}{1-(-1)} x_{0}+\left(1+\frac{(-1)}{1-(-1)}\right) x_{2}=\frac{x_{0}+x_{2}}{2}=x_{1}
$$


$\operatorname{Em} \xi=0$ :

$$
x=\frac{0}{1-0} x_{0}+\left(1+\frac{0}{1-0}\right) x_{2}=x_{2}
$$

Finalmente, em $\xi=+1$ :

$$
x=\frac{-1}{1-1} x_{0}+\left(1+\frac{1}{1-1}\right) x_{2}=\infty=x_{3}
$$

Outra característica a ser analisada se refere à coordenada global do pólo, $x_{0}$. Quando $\xi$ tende a $\infty$, o ponto $x$ tende ao pólo. Isto é demonstrado abaixo:

$$
\lim _{\xi \rightarrow \infty}[x(\xi)]=\lim _{\xi \rightarrow \infty}\left[\tilde{N}_{0}(\xi)\right] x_{0}+\lim _{\xi \rightarrow \infty}\left[\tilde{N}_{2}(\xi)\right] x_{2}
$$

Os limites do lado direito da igualdade podem ser facilmente calculados como segue:

$$
\begin{gathered}
\lim _{\xi \rightarrow \infty}\left[\tilde{N}_{0}(\xi)\right]=\lim _{\xi \rightarrow \infty}\left[\frac{-\xi}{1-\xi}\right]=1 \\
\lim _{\xi \rightarrow \infty}\left[\tilde{N}_{2}(\xi)\right]=\lim _{\xi \rightarrow \infty}\left[1+\frac{\xi}{1-\xi}\right]=1-1=0
\end{gathered}
$$

Portanto:

$$
\lim _{\xi \rightarrow \infty}[x(\xi)]=(1) x_{0}+(0) x_{2}=x_{0}
$$

Nota-se que $\tilde{N}_{0}+\tilde{N}_{2}=1$ para qualquer $\xi$. Esta é uma importante característica destas funções, e caso não fosse satisfeita o mapeamento seria afetado por uma mudança na origem do sistema de coordenadas. Isto pode ser verificado promovendo um deslocamento $\Delta x$ qualquer na origem. Isto implica nas seguintes alterações de coordenadas:

$$
x_{0}^{*}=x_{0}+\Delta x, \quad x_{2}^{*}=x_{2}+\Delta x
$$

Além disto, qualquer que seja $\Delta x$, é necessário que:

$$
x^{*}=x+\Delta x
$$

Substituindo estas expressões na equação 4.26 .

$$
x+\Delta x=\tilde{N}_{0}\left(x_{0}+\Delta x\right)+\tilde{N}_{2}\left(x_{2}+\Delta x\right) \rightarrow \Delta x=\Delta x\left(\tilde{N}_{0}+\tilde{N}_{2}\right)
$$


Para que isto seja verdade, é necessário que $\tilde{N}_{0}+\tilde{N}_{2}=1$.

Foi então estabelecido um mapeamento entre um domínio infinito e um finito por meio da igualdade 4.26. Pode-se ainda encontrar uma expressão mais conveniente substituindose as expressões 4.27 em 4.26, O resultado é a igualdade:

$$
x=x_{0}+\frac{2 a}{1-\xi}
$$

que indica como encontrar a coordenada $x$ a partir da coordenada $\xi$, sendo a dimensão $a$ indicada na figura 4.2. Caso se queira $\xi$ em função de $x$ basta trabalhar a expressão 4.38 , obtendo:

$$
\xi=1-\frac{2 a}{x-x_{0}}
$$

Considerando ainda que $r=x-x_{0}$ (figura 4.2), as equações 4.38 e 4.39 se tornam:

$$
r=\frac{2 a}{1-\xi}
$$

$\mathrm{e}$

$$
\xi=1-\frac{2 a}{r}
$$

O próximo passo é verificar como as funções de forma de um elemento finito de origem, válidas para um domínio finito $\xi$, se comportam quando mapeadas para o domínio infinito $x$. Sabe-se que, quase sempre, as funções de forma empregadas em elementos finitos são polinomiais. Considera-se assim, como exemplo, uma expressão polinomial na forma:

$$
P(\xi)=\alpha_{0}+\alpha_{1} \xi+\alpha_{2} \xi^{2}+\alpha_{3} \xi^{3}+\cdots
$$

Para mapear a função $P$ do domínio finito $\xi$ para o domínio infinito $r$ basta empregar a igualdade 4.40. O resultado é uma expressão do tipo:

$$
P(r)=\beta_{0}+\frac{\beta_{1}}{r}+\frac{\beta_{2}}{r^{2}}+\frac{\beta_{3}}{r^{3}}+\cdots
$$

Em 4.43, todos os $\beta_{i}$ podem ser determinados a partir dos $\alpha_{i}$ e da constante $a$. Observando-se a expressão do lado esquerdo da igualdade 4.43 percebe-se que, quando $r$ tende a infinito, o valor de $P(r)$ tende a $\beta_{0}$. Desta forma torna-se possível que a função de forma do elemento infinito tenda a qualquer valor desejado no infinito, bastando igualar 
$\beta_{0}$ a este valor.

A expressão 4.26 mapeia a coordenada homogênea $\xi$ para a coordenada $x$ em função da posição do pólo, $x_{0}$, e da posição do nó $2, x_{2}$. Em alguns aspectos, os quais serão elucidados mais adiante, torna-se mais vantajoso que o mapeamento seja feito em função da posição dos nós do elemento infinito. Este tipo de mapeamento foi feito por Marques e Owen (1984), e será apresentado a seguir. As funções de mapeamento $\tilde{N}_{0}$ e $\tilde{N}_{2}$ são substituídas pelas funções $M_{1}$ e $M_{2}$, de forma que:

$$
x=M_{1}(\xi) x_{1}+M_{2}(\xi) x_{2}
$$

Para que as funções $M_{1}$ e $M_{2}$ sejam escolhidas de forma coerente, alguns critérios devem ser adotados. Segundo Bettess (1992), as funções de mapeamento devem satisfazer às seguintes condições:

1. Para qualquer $\xi$, a soma das funções de mapeamento deve ser igual a 1.

2. Para qualquer $\xi$, a soma das derivadas das funções de mapeamento deve ser igual a zero.

3. Dada uma função de mapeamento $M_{i}$, ela deve ter valor unitário no nó $i$ e deve ser igual a zero nos demais nós para os quais são escritas funções de mapeamento.

4. Sendo $\xi_{j}$ a coordenada adimensional do nó mapeado ao infinito, todas as funções de mapeamento devem tender a $\infty$ ou $-\infty$ quando $\xi$ tende a $\xi_{j}$.

Tendo em vista todos estes fatores, escolhe-se as seguintes funções:

$$
M_{1}(\xi)=\left(\frac{\xi-\xi_{2}}{\xi_{1}-\xi_{2}}\right)\left(\frac{\xi_{1}-\xi_{3}}{\xi-\xi_{3}}\right), M_{2}(\xi)=\left(\frac{\xi-\xi_{1}}{\xi_{2}-\xi_{1}}\right)\left(\frac{\xi_{2}-\xi_{3}}{\xi-\xi_{3}}\right)
$$

Ao substituir os valores de $\xi_{1}=-1, \xi_{2}=0$ e $\xi_{3}=1$ em 4.45 , obtém-se:

$$
\begin{gathered}
M_{1}(\xi)=\frac{-2 \xi}{1-\xi}, \quad M_{2}(\xi)=\frac{1+\xi}{1-\xi} \\
\frac{\partial M_{1}}{\partial \xi}=\frac{-2}{(1-\xi)^{2}}, \quad \frac{\partial M_{2}}{\partial \xi}=\frac{2}{(1-\xi)^{2}}
\end{gathered}
$$

Pode ser facilmente demonstrado que estas funções de mapeamento satisfazem a todos os critérios enumerados anteriormente. É importante notar também que a posição do pólo 
não foi alterada, conforme demonstrado abaixo:

$$
\begin{aligned}
& \lim _{\xi \rightarrow \infty}\left[M_{1}(\xi)\right]=\lim _{\xi \rightarrow \infty}\left[\frac{-2 \xi}{1-\xi}\right]=2 \\
& \lim _{\xi \rightarrow \infty}\left[M_{2}(\xi)\right]=\lim _{\xi \rightarrow \infty}\left[\frac{1+\xi}{1-\xi}\right]=-1
\end{aligned}
$$

Portanto:

$$
\lim _{\xi \rightarrow \infty}[x(\xi)]=2 x_{1}-x_{3}=x_{1}-\left(x_{3}-x_{1}\right)=x_{1}-a=x_{0}
$$

Assim, dadas as funções de mapeamento, utiliza-se este procedimento para determinar a posição global do pólo em função das coordenadas globais dos nós do elemento infinito. É mencionado em Bettess (1992) que o ideal seria posicionar estes nós de forma que o pólo coincida com o centro de decaimento do problema. Entretanto, segundo Moser et al. (2004), é possível obter resultados precisos empregando elementos infinitos em problemas que não possuem um único centro de decaimento, ou que não têm este centro claramente definido.

O comportamento de $M_{1}$ e $M_{2}$ pode ser visualizado no gráfico da figura 4.3 .

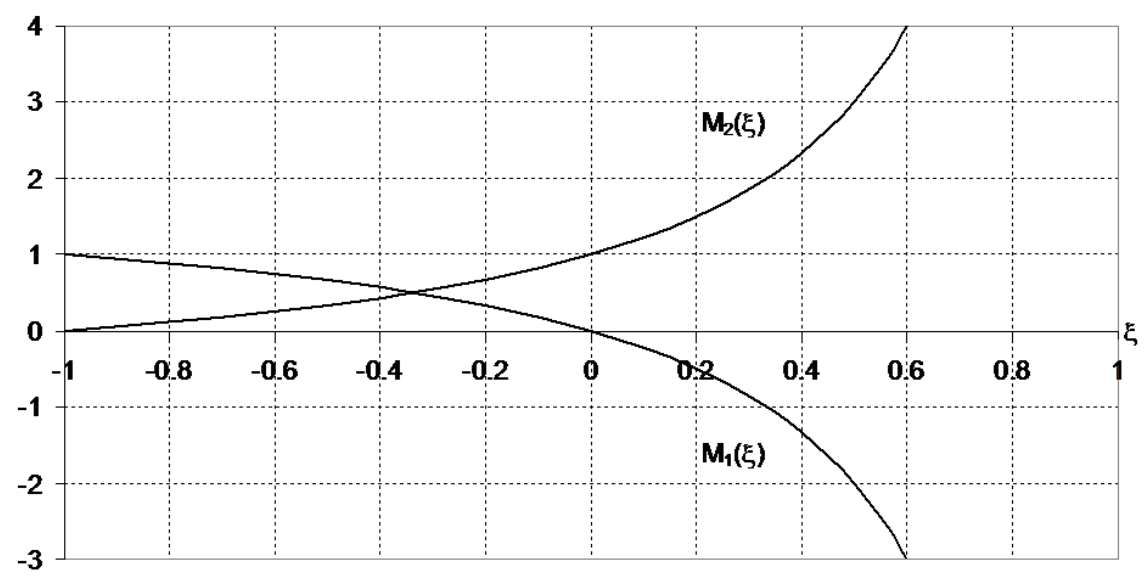

Figura 4.3: Funções de mapeamento

As vantagens do mapeamento $M_{i}$ sobre o $N_{i}$ se torna clara ao analisar-se as funções de forma $P_{i}$ do elemento finito de origem. Escrevendo $P_{1}$ e $P_{2}$ em função de $\xi_{i}$, da mesma forma que foi feito para $M_{i}$ em 4.45 , obtém-se:

$$
P_{1}(\xi)=\left(\frac{\xi-\xi_{2}}{\xi_{1}-\xi_{2}}\right)\left(\frac{\xi-\xi_{3}}{\xi_{1}-\xi_{3}}\right), \quad P_{2}(\xi)=\left(\frac{\xi-\xi_{1}}{\xi_{2}-\xi_{1}}\right)\left(\frac{\xi-\xi_{3}}{\xi_{2}-\xi_{3}}\right)
$$


Substituindo em 4.51 os valores de $\xi_{1}, \xi_{2}$ e $\xi_{3}$ :

$$
P_{1}(\xi)=\frac{\xi(\xi-1)}{2}, \quad P_{2}(\xi)=(1+\xi)(1-\xi)
$$

Comparando-se 4.51 com 4.45 é possível notar que as funções $M_{i}$ e $P_{i}$ são quase iguais, sendo a única diferença o último termo entre parênteses à direita de cada igualdade. Este termo se refere ao ponto do elemento finito o qual é mapeado ao infinito, que neste caso é o de coordenada $\xi_{3}$. Basta inverter este termo em uma função de forma $P_{i}$ para obter sua função de mapeamento $M_{i}$ correspondente. Segundo Bettess (1992), esta regra é vantajosa por tornar simples a obtenção das funções de mapeamento de um elemento infinito qualquer, bastando que sejam conhecidas as funções de forma do elemento finito de origem. Tendo isto em vista são adotadas, a partir deste ponto, somente funções de mapeamento do tipo $M_{i}$.

\subsubsection{Elemento infinito unidimensional linear}

Empregando os conceitos apresentados anteriormente são deduzidas agora funções de mapeamento para um elemento infinito unidimensional linear. A figura 4.4 ilustra o procedimento a ser feito. É importante observar que os índices sobrescritos indicam o ponto ao qual se refere a coordenada ou função, pois este critério é adotado a para o restante do Capítulo.

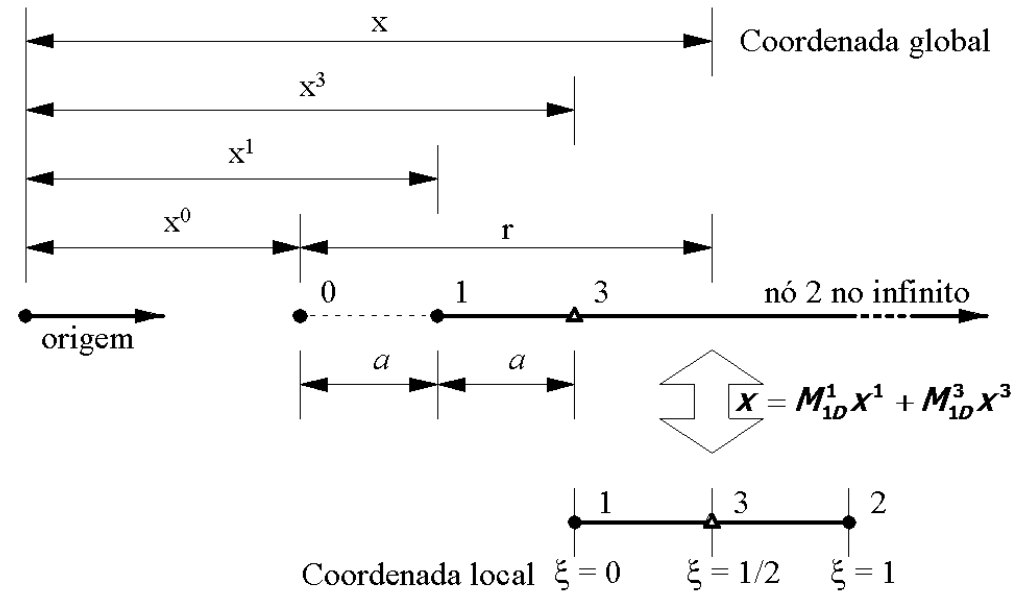

Figura 4.4: Elemento unidimensional linear

Nota-se que o elemento é semelhante ao de Zienkiewicz, sendo as únicas diferenças o grau das funções de forma e a origem da coordenada $\xi$. Esta é convenientemente 
posicionada em um dos nós do elemento, para que seja possível empregar, posteriormente, as expressões aqui definidas na dedução de um elemento bidimensional. O sistema de coordenadas global é relacionado ao local empregando funções de mapeamento. Como o nó 2 está posicionado no infinito ele não possui função de forma nem contribui na transformação de coordenadas, então é definido um nó de referência posicionado no ponto central do elemento e identificado como 3 na numeração local. O pólo está posicionado na coordenada global $x=x^{0}$.

A única função do nó 3 é servir de referência às funções de mapeamento portanto, assim como o nó 2 , ele não possui função de forma. Basta então definir a função de forma do nó 1 , que é a única a ser integrada no elemento infinito:

$$
P^{1}(\xi)=1-\xi
$$

O nó 1, por ser o único a possuir função de forma, recebe toda a contribuição do elemento infinito. Observando a figura 4.4 e imaginando que o lado esquerdo está conectado a uma malha de elementos finitos conclui-se que, neste caso, nenhum grau de liberdade é adicionado à malha pela presença do elemento infinito. $\mathrm{O}$ elemento de contorno infinito bidimensional que é empregado neste trabalho também possui esta vantagem, conforme será visto mais adiante.

São deduzidas agora as funções de mapeamento dos nós 1 e 3. Para obter estas funções, emprega-se expressões análogas às igualdades 4.45, atentando à numeração dos nós e às suas coordenadas. Assim, sendo $\xi^{i}$ a coordenada $\xi$ do nó $i$ :

$$
M_{1 D}^{1}(\xi)=\left(\frac{\xi-\xi^{3}}{\xi^{1}-\xi^{3}}\right)\left(\frac{\xi^{1}-\xi^{2}}{\xi-\xi^{2}}\right), M_{1 D}^{3}(\xi)=\left(\frac{\xi-\xi^{1}}{\xi^{3}-\xi^{1}}\right)\left(\frac{\xi^{3}-\xi^{2}}{\xi-\xi^{2}}\right)
$$

É colocado subscrito o texto " $1 D$ " para indicar que estas funções de mapeamento são válidas para um elemento unidimensional. Sabendo que $\xi^{1}=0, \xi^{2}=1$ e $\xi^{3}=1 / 2$, tem-se que:

$$
\begin{gathered}
M_{1 D}^{1}(\xi)=\frac{1-2 \xi}{1-\xi}, \quad M_{1 D}^{3}(\xi)=\frac{\xi}{1-\xi} \\
\frac{\partial M_{1 D}^{1}}{\partial \xi}=\frac{-1}{(1-\xi)^{2}}, \quad \frac{\partial M_{1 D}^{3}}{\partial \xi}=\frac{1}{(1-\xi)^{2}}
\end{gathered}
$$

Para avaliar se estas funções de mapeamento são adequadas considera-se as mesmas verificações aplicadas para o elemento infinito de Zienkiewicz, ou seja: 
1. Para qualquer $\xi$, a soma das funções de mapeamento deve ser igual a 1 .

$$
M_{1 D}^{1}(\xi)+M_{1 D}^{3}(\xi)=\frac{1-2 \xi}{1-\xi}+\frac{\xi}{1-\xi}=\frac{1-\xi}{1-\xi}=1 \rightarrow O K !
$$

2. Para qualquer $\xi$, a soma das derivadas das funções de mapeamento deve ser igual a zero.

$$
\frac{\partial M_{1 D}^{1}}{\partial \xi}+\frac{\partial M_{1 D}^{3}}{\partial \xi}=\frac{-1}{(1-\xi)^{2}}+\frac{1}{(1-\xi)^{2}}=0 \rightarrow O K !
$$

3. Para $\xi=0$, a função $M_{1 D}^{1}$ deve ter valor unitário e a função $M_{1 D}^{3}$ deve ser igual a zero.

$$
\begin{gathered}
M_{1 D}^{1}(0)=\frac{1-2 \times 0}{1-0}=\frac{1}{1}=1 \rightarrow O K ! \\
M_{1 D}^{3}(0)=\frac{0}{1-0}=\frac{0}{1}=0 \rightarrow O K !
\end{gathered}
$$

4. Para $\xi=1 / 2$, a função $M_{1 D}^{1}$ deve ser igual a zero e a função $M_{1 D}^{3}$ deve ter valor unitário.

$$
\begin{gathered}
M_{1 D}^{1}(1 / 2)=\frac{1-2(1 / 2)}{1-(1 / 2)}=\frac{1-1}{1 / 2}=0 \rightarrow O K ! \\
M_{1 D}^{3}(1 / 2)=\frac{1 / 2}{1-1 / 2}=\frac{1 / 2}{1 / 2}=1 \rightarrow O K !
\end{gathered}
$$

5. Quando $\xi$ tende a 1 , a função $M_{1 D}^{1}$ deve tender a $-\infty$ e a função $M_{1 D}^{3}$ deve tender a $\infty$.

$$
\begin{gathered}
M_{1 D}^{1}(1)=\frac{1-2(1)}{1-1}=\frac{-1}{0}=-\infty \rightarrow O K ! \\
M_{1 D}^{3}(1)=\frac{1}{1-1}=\frac{1}{0}=\infty \rightarrow O K !
\end{gathered}
$$

Obtidas as funções de mapeamento, torna-se possível relacionar o sistema de coordenadas global ao sistema homogêneo local. Isto é:

$$
x(\xi)=M_{1 D}^{1}(\xi) x^{1}+M_{1 D}^{3}(\xi) x^{3}
$$

As funções $M_{1 D}^{1}(\xi)$ e $M_{1 D}^{3}(\xi)$ são definidas nas igualdades 4.55 , e $x^{1}$ e $x^{3}$ correspondem às coordenadas globais dos nós 1 e 3 , respectivamente. Para determinar a posição do pólo, conforme apresentado anteriormente, considera-se o limite desta função quando $\xi$ tende a 
$\infty$. Assim:

$$
\begin{aligned}
& \lim _{\xi \rightarrow \infty}\left[M_{1 D}^{1}(\xi)\right]=\lim _{\xi \rightarrow \infty}\left[\frac{1-2 \xi}{1-\xi}\right]=2 \\
& \lim _{\xi \rightarrow \infty}\left[M_{1 D}^{3}(\xi)\right]=\lim _{\xi \rightarrow \infty}\left[\frac{\xi}{1-\xi}\right]=-1
\end{aligned}
$$

Portanto:

$$
\lim _{\xi \rightarrow \infty}[x(\xi)]=2 x^{1}-x^{3}=x^{1}-\left(x^{3}-x^{1}\right)=x^{1}-a=x^{0}
$$

em que $a$ é a distância do pólo ao nó 1 e também do nó 1 ao nó 3 , conforme apresentado na figura 4.4. Para uma análise mais detalhada do mapeamento, substitui-se as expressões 4.55 em 4.57. Assim:

$$
x(\xi)=x^{1}+\frac{\xi a}{1-\xi}
$$

Caso se queira relacionar $r$, indicado na figura 4.4 , com a coordenada homogênea $\xi$, é preciso primeiramente escrever $r$ em função de $x$. Pela figura, observa-se que esta relação é, simplesmente:

$$
r=x-x^{0}
$$

Portanto, subtraindo-se $x^{0}$ em ambos os lados da equação 4.61, obtém-se:

$$
r=\frac{a}{1-\xi}
$$

e

$$
\xi=1-\frac{a}{r}
$$

Finalmente calcula-se o Jacobiano a partir das funções $M_{1 D}^{1}(\xi)$ e $M_{1 D}^{3}(\xi)$. Este Jacobiano é dado por:

$$
\left.\right|^{\infty} J \mid=\frac{d M_{1 D}^{1}}{d \xi} x^{1}+\frac{d M_{1 D}^{3}}{d \xi} x^{3}=\frac{x^{3}-x^{1}}{(1-\xi)^{2}}=\frac{a}{(1-\xi)^{2}}
$$

em que o símbolo " $\infty$ " sobrescrito à esquerda indica que o Jacobiano se refere a um elemento infinito. Assim, utilizando as mesmas funções de forma e mantendo os pontos de integração inalterados, o elemento finito de origem se torna infinito pela substituição do Jacobiano original pelo dado pela igualdade 4.65 .

É interessante ainda avaliar como o Jacobiano varia em função de $r$. Desta forma, 
substituindo em 4.65 a igualdade 4.64, obtém-se:

$$
\left.\right|^{\infty} J \mid=\frac{r^{2}}{a}
$$

Portanto, o Jacobiano deste mapeamento tem ordem $r^{2}$.

\subsection{Elemento de contorno infinito bidimensional}

Após avaliar algumas alternativas optou-se, neste trabalho, pela criação de um elemento de contorno infinito (ECI) com funções de mapeamento. É empregado como origem o elemento de contorno (EC) triangular com funções de forma lineares que é descrito no capítulo 2. Diversas vantagens justificam esta escolha, conforme será apresentado ao longo desta Seção.

\subsubsection{Definição das funções de mapeamento}

São considerados três tipos de mapeamento. No primeiro deles somente a direção $\xi_{1}$ é mapeada, conforme ilustrado na figura 4.5 .

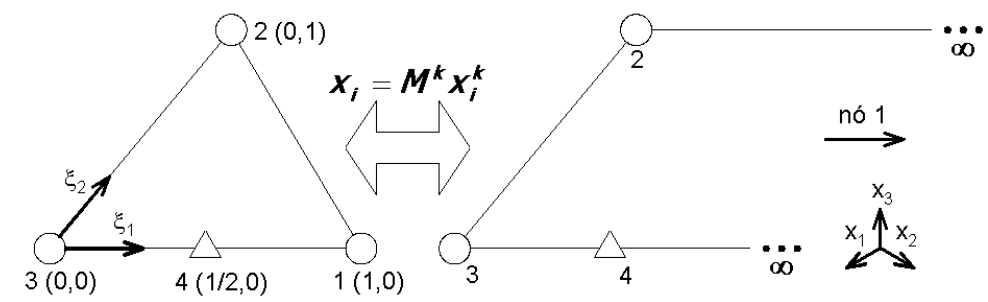

Figura 4.5: Mapeamento da direção $\xi_{1}$

Dada uma direção $i$ no sistema de coordenadas retangular global, a coordenada $x_{i}$ é escrita em função das coordenadas globais do EC de origem, $x_{i}^{k}$, e das funções de mapeamento $M^{k}$. No lado esquerdo da figura é indicado o EC de origem, com a numeração local dos nós e suas respectivas coordenadas homogêneas. No lado direito é apresentado o ECI, que é definido no sistema de coordenadas global, sendo que a numeração de cada nó indica o correspondente do EC de origem. Conforme pode ser observado na figura, somente o nó 1 foi mapeado ao infinito. Por este motivo este nó não possui função de mapeamento, além de não possuir função de forma. Após analisar algumas alternativas, foi verificado que surgem problemas com o Jacobiano caso sejam utilizados somente dois 
nós para mapear o ECI. Para contornar este problema é criado um quarto nó, indicado na figura como nó 4, cuja função é unicamente auxiliar no mapeamento. Desta forma, o nó 4 possui função de mapeamento mas não possui função de forma. Isto significa que somente os nós 2 e 3 contribuem quando este ECI é integrado.

O segundo tipo de mapeamento a ser considerado é quando somente a direção $\xi_{2}$ é mapeada. Isto é apresentado na figura 4.6.

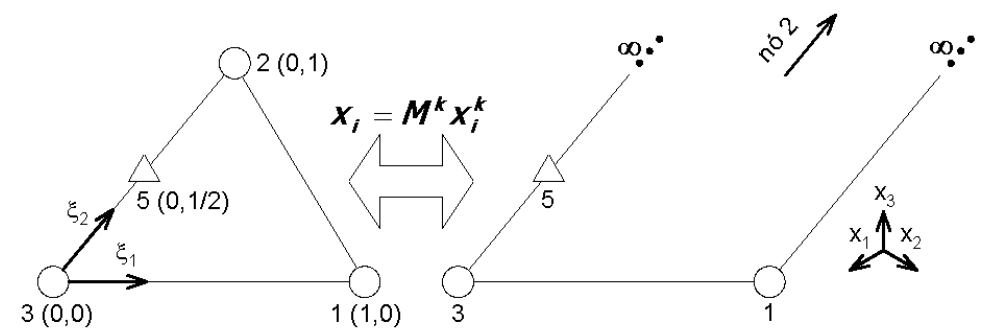

Figura 4.6: Mapeamento da direção $\xi_{2}$

Assim como na figura anterior são representados, na figura 4.6, o EC de origem e o ECI. Neste caso somente o nó 2 foi mapeado ao infinito, portanto este nó não possui função de mapeamento nem função de forma. Se torna necessária, novamente, a criação de um nó auxiliar para o mapeamento, o qual é indicado na figura como nó 5 . Como o nó auxiliar não possui função de forma, no caso do mapeamento da direção $\xi_{2}$ somente os nós 1 e 3 contribuem na integral do elemento.

No terceiro e último caso as duas direções, $\xi_{1}$ e $\xi_{2}$, são mapeadas. A figura 4.7 ilustra a situação.

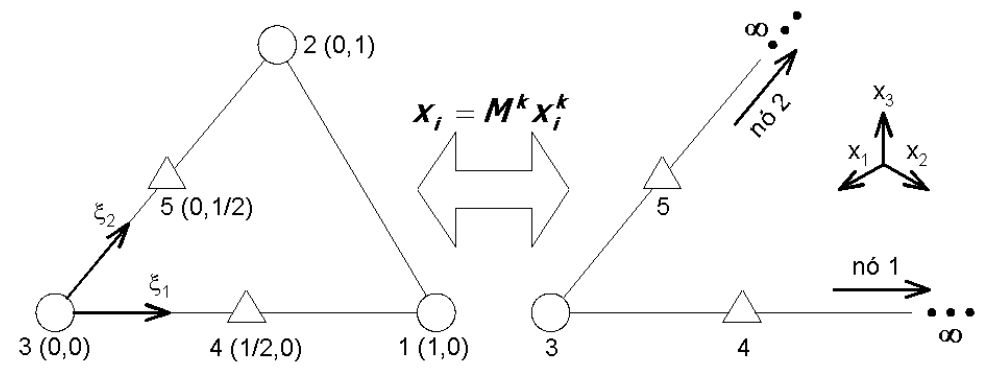

Figura 4.7: Mapeamento das direções $\xi_{1}$ e $\xi_{2}$

Assim como nas figuras anteriores, na figura 4.7 são representados o EC e o ECI. Como neste caso dois nós são mapeados ao infinito, torna-se necessária a criação de dois nós auxiliares. Estes nós são indicados na figura com os números 4 e 5, e assim como anteriormente eles possuem função de mapeamento mas não possuem função de forma. 
Portanto, para mapeamento em duas direções, somente o nó 3 contribui na integral do elemento.

O passo seguinte é a definição das funções de mapeamento. Como o sistema de coordenadas local é oblíquo, neste trabalho isto é feito empregando uma estratégia diferente das abordagens difundidas na literatura. Sendo $\xi_{i}$ uma direção a ser mapeada, é criada uma coordenada auxiliar $\bar{\xi}_{i}\left(\xi_{i}\right)$ com a função de definir este mapeamento. A escolha da função $\bar{\xi}_{i}\left(\xi_{i}\right)$ foi fundamentada no trabalho de Davis e Rabinowitz (1975), resultando na seguinte expressão:

$$
\bar{\xi}_{i}=\frac{\xi_{i}}{1-\xi_{i}}
$$

Portanto, caso se queira mapear a direção $\xi_{1}$, escreve-se:

$$
\bar{\xi}_{1}\left(\xi_{1}\right)=\frac{\xi_{1}}{1-\xi_{1}}
$$

e para mapear a direção $\xi_{2}$ :

$$
\bar{\xi}_{2}\left(\xi_{2}\right)=\frac{\xi_{2}}{1-\xi_{2}}
$$

Definidas as igualdades 4.68 e 4.69 , a estratégia para a obtenção das funções de mapeamento consiste em substituir estas expressões nas funções de forma do EC de origem. Conforme apresentado no capítulo 2, estas funções de forma são:

$$
\begin{gathered}
\phi^{1}=\xi_{1} \\
\phi^{2}=\xi_{2} \\
\phi^{3}=1-\xi_{1}-\xi_{2}
\end{gathered}
$$

Desta forma, caso se queira mapear somente a direção $\xi_{1}$, figura 4.5, substitui-se somente a igualdade 4.68 nas funções $4.70,4.71$ e 4.72 . O resultado é:

$$
\begin{gathered}
M_{1 \infty}^{4}=\bar{\xi}_{1}\left(\xi_{1}\right)=\frac{\xi_{1}}{1-\xi_{1}}, \quad \frac{\partial M_{1 \infty}^{4}}{\partial \xi_{1}}=\frac{1}{\left(1-\xi_{1}\right)^{2}}, \quad \frac{\partial M_{1 \infty}^{4}}{\partial \xi_{2}}=0 \\
M_{1 \infty}^{2}=\xi_{2}, \quad \frac{\partial M_{\infty 1}^{2}}{\partial \xi_{1}}=0, \quad \frac{\partial M_{\infty 1}^{2}}{\partial \xi_{2}}=1 \\
M_{1 \infty}^{3}=1-\bar{\xi}_{1}\left(\xi_{1}\right)-\xi_{2}=1-\frac{\xi_{1}}{1-\xi_{1}}-\xi_{2}, \quad \frac{\partial M_{1 \infty}^{3}}{\partial \xi_{1}}=\frac{-1}{\left(1-\xi_{1}\right)^{2}}, \quad \frac{\partial M_{1 \infty}^{3}}{\partial \xi_{2}}=-1
\end{gathered}
$$


O símbolo " $1 \infty$ " subscrito indica que somente a direção $\xi_{1}$ foi mapeada. É importante observar que o nó 1 não possui função de mapeamento, sendo substituído pelo nó auxiliar 4. Também deve ser salientado que a função de mapeamento $M_{1 \infty}^{2}$ é exatamente igual à função de forma original. Isto significa que a direção $\xi_{2}$ não sofreu influência do mapeamento.

Para mapear somente a direção $\xi_{2}$, figura 4.6, substitui-se somente 4.69 nas funções 4.70, 4.71 e 4.72. Ou seja:

$$
\begin{gathered}
M_{2 \infty}^{1}=\xi_{1}, \quad \frac{\partial M_{2 \infty}^{1}}{\partial \xi_{1}}=1, \quad \frac{\partial M_{2 \infty}^{1}}{\partial \xi_{2}}=0 \\
M_{2 \infty}^{5}=\bar{\xi}_{2}\left(\xi_{2}\right)=\frac{\xi_{2}}{1-\xi_{2}}, \frac{\partial M_{2 \infty}^{5}}{\partial \xi_{1}}=0, \quad \frac{\partial M_{2 \infty}^{5}}{\partial \xi_{2}}=\frac{1}{\left(1-\xi_{2}\right)^{2}} \\
M_{2 \infty}^{3}=1-\xi_{1}-\bar{\xi}_{2}\left(\xi_{2}\right)=1-\xi_{1}-\frac{\xi_{2}}{1-\xi_{2}}, \frac{\partial M_{2 \infty}^{3}}{\partial \xi_{1}}=-1, \quad \frac{\partial M_{2 \infty}^{3}}{\partial \xi_{2}}=\frac{-1}{\left(1-\xi_{2}\right)^{2}}
\end{gathered}
$$

Empregou-se o símbolo " $2 \infty$ ” subscrito para indicar que somente a direção $\xi_{2}$ foi mapeada. Neste caso é o nó 2 que é substituído por um nó auxiliar, o 5, e a função $M_{2 \infty}^{1}$ é extamente igual à função de forma original. Portanto, a direção $\xi_{1}$ não é influenciada pelo mapeamento.

O terceiro e último caso tratado neste trabalho é quando ambas as direções, $\xi_{1}$ e $\xi_{2}$, são mapeadas, conforme ilustrado na figura 4.7. Deve-se então substituir 4.68 e 4.69 nas funções 4.70, 4.71 e 4.72 , obtendo:

$$
\begin{gathered}
M_{\infty}^{4}=\bar{\xi}_{1}\left(\xi_{1}\right)=\frac{\xi_{1}}{1-\xi_{1}}, \quad \frac{\partial M_{\infty}^{4}}{\partial \xi_{1}}=\frac{1}{\left(1-\xi_{1}\right)^{2}}, \quad \frac{\partial M_{\infty}^{4}}{\partial \xi_{2}}=0 \\
M_{\infty}^{5}=\bar{\xi}_{2}\left(\xi_{2}\right)=\frac{\xi_{2}}{1-\xi_{2}}, \quad \frac{\partial M_{\infty}^{5}}{\partial \xi_{1}}=0, \quad \frac{\partial M_{\infty}^{5}}{\partial \xi_{2}}=\frac{1}{\left(1-\xi_{2}\right)^{2}} \\
M_{\infty}^{3}=1-\bar{\xi}_{1}\left(\xi_{1}\right)-\bar{\xi}_{2}\left(\xi_{2}\right)=1-\frac{\xi_{1}}{1-\xi_{1}}-\frac{\xi_{2}}{1-\xi_{2}}, \quad \frac{\partial M_{\infty}^{3}}{\partial \xi_{1}}=\frac{-1}{\left(1-\xi_{1}\right)^{2}}, \quad \frac{\partial M_{\infty}^{3}}{\partial \xi_{2}}=\frac{-1}{\left(1-\xi_{2}\right)^{2}}
\end{gathered}
$$

Neste terceiro caso indicou-se subscrito simplesmente o símbolo " $\infty$ ", pois abmas as direções são mapeadas. Os nós 1 e 2 são substituídos pelos auxiliares 4 e 5 , respectivamente, para mapear o elemento.

Definidas as funções de mapeamento, elas dever ser utilizadas para relacionar o sistema de coordenadas global ao sistema local do elemento. Assim, para decaimento somente na 
direção $\xi_{1}$ :

$$
x_{i}=M_{1 \infty}^{4} x_{i}^{4}+M_{1 \infty}^{2} x_{i}^{2}+M_{1 \infty}^{3} x_{i}^{3}
$$

Para decaimento na direção $\xi_{2}$ :

$$
x_{i}=M_{2 \infty}^{1} x_{i}^{1}+M_{2 \infty}^{5} x_{i}^{5}+M_{2 \infty}^{3} x_{i}^{3}
$$

E para decaimento nas duas direções:

$$
x_{i}=M_{\infty}^{4} x_{i}^{4}+M_{\infty}^{5} x_{i}^{5}+M_{\infty}^{3} x_{i}^{3}
$$

\subsubsection{Verificação das funções de mapeamento}

Após definir as funções de mapeamento bidimensionais, é necessário verificar se suas características são adequadas. Isto é feito aplicando as mesmas regras consideradas em seções anteriores para funções de mapeamento unidimensionais. Estas regras são enumeradas a seguir:

1. Para quaisquer $\left(\xi_{1}, \xi_{2}\right)$, a soma das funções de mapeamento deve ser igual a 1 .

$$
\begin{gathered}
M_{1 \infty}^{4}+M_{1 \infty}^{2}+M_{1 \infty}^{3}=\frac{\xi_{1}}{1-\xi_{1}}+\xi_{2}+1-\frac{\xi_{1}}{1-\xi_{1}}-\xi_{2}=1 \quad \rightarrow \quad O K ! \\
M_{2 \infty}^{1}+M_{2 \infty}^{5}+M_{2 \infty}^{3}=\xi_{1}+\frac{\xi_{2}}{1-\xi_{2}}+1-\xi_{1}-\frac{\xi_{2}}{1-\xi_{2}}=1 \quad \rightarrow \quad O K ! \\
M_{\infty}^{4}+M_{\infty}^{5}+M_{\infty}^{3}=\frac{\xi_{1}}{1-\xi_{1}}+\frac{\xi_{2}}{1-\xi_{2}}+1-\frac{\xi_{1}}{1-\xi_{1}}-\frac{\xi_{2}}{1-\xi_{2}}=1 \quad \rightarrow \quad O K !
\end{gathered}
$$

2. Para quaisquer $\left(\xi_{1}, \xi_{2}\right)$, a soma das derivadas das funções de mapeamento deve ser igual a zero.

$$
\begin{gathered}
\frac{\partial M_{1 \infty}^{4}}{\partial \xi_{1}}+\frac{\partial M_{1 \infty}^{2}}{\partial \xi_{1}}+\frac{\partial M_{1 \infty}^{3}}{\partial \xi_{1}}=\frac{1}{\left(1-\xi_{1}\right)^{2}}+0-\frac{1}{\left(1-\xi_{1}\right)^{2}}=0 \quad \rightarrow \quad O K ! \\
\frac{\partial M_{1 \infty}^{4}}{\partial \xi_{2}}+\frac{\partial M_{1 \infty}^{2}}{\partial \xi_{2}}+\frac{\partial M_{1 \infty}^{3}}{\partial \xi_{2}}=0+1-1=0 \quad \rightarrow \quad O K ! \\
\frac{\partial M_{2 \infty}^{1}}{\partial \xi_{1}}+\frac{\partial M_{2 \infty}^{5}}{\partial \xi_{1}}+\frac{\partial M_{2 \infty}^{3}}{\partial \xi_{1}}=1+0-1=0 \quad \rightarrow \quad O K !
\end{gathered}
$$




$$
\begin{gathered}
\frac{\partial M_{2 \infty}^{1}}{\partial \xi_{2}}+\frac{\partial M_{2 \infty}^{5}}{\partial \xi_{2}}+\frac{\partial M_{2 \infty}^{3}}{\partial \xi_{2}}=0+\frac{1}{\left(1-\xi_{2}\right)^{2}}-\frac{1}{\left(1-\xi_{2}\right)^{2}}=0 \quad \rightarrow \quad O K ! \\
\frac{\partial M_{\infty}^{4}}{\partial \xi_{1}}+\frac{\partial M_{\infty}^{5}}{\partial \xi_{1}}+\frac{\partial M_{\infty}^{3}}{\partial \xi_{1}}=\frac{1}{\left(1-\xi_{1}\right)^{2}}+0-\frac{1}{\left(1-\xi_{1}\right)^{2}}=0 \quad \rightarrow \quad O K ! \\
\frac{\partial M_{\infty}^{4}}{\partial \xi_{2}}+\frac{\partial M_{\infty}^{5}}{\partial \xi_{2}}+\frac{\partial M_{\infty}^{3}}{\partial \xi_{2}}=0+\frac{1}{\left(1-\xi_{2}\right)^{2}}-\frac{1}{\left(1-\xi_{2}\right)^{2}}=0 \quad \rightarrow \quad O K !
\end{gathered}
$$

3. Dada uma função de mapeamento $M^{i}$, ela deve ter valor unitário no nó $i$ e deve ser igual a zero nos demais nós para os quais são escritas funções de mapeamento.

$$
\begin{aligned}
& M_{1 \infty}^{4}(1 / 2,0)=1, \quad M_{1 \infty}^{4}(0,1)=0, \quad M_{1 \infty}^{4}(0,0)=0 \rightarrow O K ! \\
& M_{1 \infty}^{2}(1 / 2,0)=0, \quad M_{1 \infty}^{2}(0,1)=1, \quad M_{1 \infty}^{2}(0,0)=0 \rightarrow O K ! \\
& M_{1 \infty}^{3}(1 / 2,0)=0, \quad M_{1 \infty}^{3}(0,1)=0, \quad M_{1 \infty}^{3}(0,0)=1 \quad \rightarrow \quad O K ! \\
& M_{2 \infty}^{1}(1,0)=1, \quad M_{2 \infty}^{1}(0,1 / 2)=0, \quad M_{2 \infty}^{1}(0,0)=0 \rightarrow O K ! \\
& M_{2 \infty}^{5}(1,0)=0, \quad M_{2 \infty}^{5}(0,1 / 2)=1, \quad M_{2 \infty}^{5}(0,0)=0 \quad \rightarrow \quad O K ! \\
& M_{2 \infty}^{3}(1,0)=0, \quad M_{2 \infty}^{3}(0,1 / 2)=0, \quad M_{2 \infty}^{3}(0,0)=1 \quad \rightarrow \quad O K ! \\
& M_{\infty}^{4}(1 / 2,0)=1, \quad M_{\infty}^{4}(0,1 / 2)=0, \quad M_{\infty}^{4}(0,0)=0 \quad \rightarrow \quad O K ! \\
& M_{\infty}^{5}(1 / 2,0)=0, \quad M_{\infty}^{5}(0,1 / 2)=1, \quad M_{\infty}^{5}(0,0)=0 \quad \rightarrow \quad O K ! \\
& M_{\infty}^{3}(1 / 2,0)=0, \quad M_{\infty}^{3}(0,1 / 2)=0, \quad M_{\infty}^{3}(0,0)=1 \quad \rightarrow \quad O K !
\end{aligned}
$$

4. Sendo $\left(\xi_{1}^{j}, \xi_{2}^{j}\right)$ as coordenadas adimensionais do nó mapeado ao infinito, todas as funções de mapeamento devem tender a $\infty$ ou $-\infty$ quando $\left(\xi_{1}, \xi_{2}\right)$ tende a $\left(\xi_{1}^{j}, \xi_{2}^{j}\right)$. Esta regra se aplica somente caso a função de mapeamento seja diferente da função de forma inicial do EC de origem.

$$
M_{1 \infty}^{4}(1,0)=\frac{1}{1-1}=\infty \quad \rightarrow \quad O K !
$$




$$
\begin{gathered}
M_{1 \infty}^{3}(1,0)=1-\frac{1}{1-1}-0=-\infty \rightarrow O K ! \\
M_{2 \infty}^{5}(0,1)=\frac{1}{1-1}=\infty \rightarrow O K ! \\
M_{2 \infty}^{3}(0,1)=1-0-\frac{1}{1-1}=-\infty \rightarrow O K ! \\
M_{\infty}^{4}(1,0)=\frac{1}{1-1}=\infty \rightarrow O K ! \\
M_{\infty}^{5}(0,1)=\frac{1}{1-1}=\infty \rightarrow O K ! \\
M_{\infty}^{3}(1,0)=1-0-\frac{1}{1-1}=-\infty \rightarrow O K !
\end{gathered}
$$

Portanto, as funções de mapeamento atendem a todos os quesitos necessários.

\subsubsection{Determinação da posição do pólo}

A seguir, é feito um estudo para determinar as coordenadas globais do pólo em função das coordenadas globais dos nós do ECI. Isto é feito a partir das expressões $4.82,4.83$ e 4.84. calculando seu limite quando a coordenada local mapeada tende a infinito. Assim, sendo $x_{i}^{p}$ a coordenada global $i$ do pólo e para mapeamento somente na direção $\xi_{1}$ :

$$
\begin{gathered}
x_{i}^{p}=\lim _{\xi_{1} \rightarrow \infty}\left[M_{1 \infty}^{4} x_{i}^{4}+M_{1 \infty}^{2} x_{i}^{2}+M_{1 \infty}^{3} x_{i}^{3}\right] \\
\lim _{\xi_{1} \rightarrow \infty}\left[M_{1 \infty}^{4}\right]=\lim _{\xi_{1} \rightarrow \infty}\left[\frac{\xi_{1}}{1-\xi_{1}}\right]=-1 \\
\lim _{\xi_{1} \rightarrow \infty}\left[M_{1 \infty}^{2}\right]=\lim _{\xi_{1} \rightarrow \infty}\left[\xi_{2}\right]=\xi_{2} \\
\lim _{\xi_{1} \rightarrow \infty}\left[M_{1 \infty}^{3}\right]=\lim _{\xi_{1} \rightarrow \infty}\left[1-\frac{\xi_{1}}{1-\xi_{1}}-\xi_{2}\right]=2-\xi_{2} \\
x_{i}^{p}=-x_{i}^{4}+\xi_{2} x_{i}^{2}+\left(2-\xi_{2}\right) x_{i}^{3}=x_{i}^{3}-\left(x_{i}^{4}-x_{i}^{3}\right)+\xi_{2}\left(x_{i}^{2}-x_{i}^{3}\right)
\end{gathered}
$$

Na equação 4.89, do lado direito da última igualdade, o primeiro termo entre parênteses representa a projeção em $x_{i}$ do segmento entre os nós 3 e 4 do ECI. O segundo termo entre parênteses, que multiplica $\xi_{2}$, corresponde à projeção em $x_{i}$ do segmento entre os 
nós 3 e 2 do ECI. Para simplificar a equação, utiliza-se a seguinte notação:

$$
x_{i}^{j k}=x_{i}^{j}-x_{i}^{k}
$$

Aplicando esta notação, a equação 4.89 se torna:

$$
x_{i}^{p}=x_{i}^{3}-x_{i}^{43}+\xi_{2} x_{i}^{23}
$$

Conforme pode ser observado na equação 4.91, a posição do pólo depende da coordenada $\xi_{2}$. Isto acontece porque, como somente uma direção foi mapeada, o pólo não é único. A figura 4.8 ilustra a situação a ser analisada.

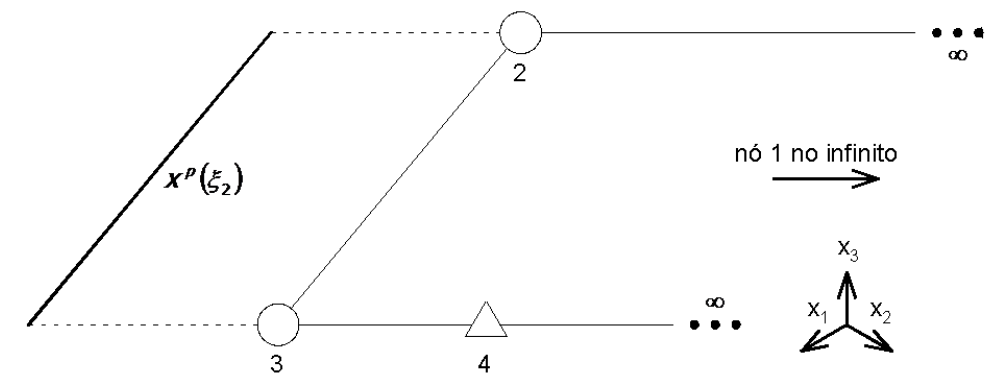

Figura 4.8: Curva que representa o pólo

A curva $x^{p}\left(\xi_{2}\right)$ representa todas as possíveis posições do pólo, de acordo com a coordenada $\xi_{2}$. Esta informação deve ser observada quando a malha de ECI for gerada pois, segundo Moser et al. (2004), mesmo quando o pólo não é único, recomenda-se que a curva que o representa seja posicionada próxima ao centro de decaimento do problema.

A seguir, determina-se a posição do pólo para mapeamento na direção $\xi_{2}$. Portanto, considera-se o limite da expressão 4.83 quando a coordenada $\xi_{2}$ tende ao infinito. Como o procedimento é análogo ao realizado anteriormente apresenta-se diretamente a expressão da coordenada $x_{i}^{p}$ do pólo, que é:

$$
x_{i}^{p}=x_{i}^{3}-\left(x_{i}^{5}-x_{i}^{3}\right)+\xi_{1}\left(x_{i}^{1}-x_{i}^{3}\right)
$$

Empregando a notação definida na igualdade 4.90, escreve-se:

$$
x_{i}^{p}=x_{i}^{3}-x_{i}^{53}+\xi_{1} x_{i}^{13}
$$


A figura 4.8 também é válida para este caso, bastando trocar a direção mapeada. Desta forma, o procedimento para o posicionamento de um ECI deste tipo é análogo ao especificado para a outra direção.

Resta ainda determinar a posição do pólo quando as duas direções, $\xi_{1}$ e $\xi_{2}$, são mapeadas. Para isto, é necessário considerar o limite da expressão 4.84 quando tanto $\xi_{1}$ como $\xi_{2}$ tendem ao infinito. Este procedimento é desenvolvido a seguir:

$$
\begin{gathered}
x_{i}^{p}=\lim _{\xi_{1}, \xi_{2} \rightarrow \infty}\left[M_{\infty}^{4} x_{i}^{4}+M_{\infty}^{5} x_{i}^{5}+M_{\infty}^{3} x_{i}^{3}\right] \\
\lim _{\xi_{1}, \xi_{2} \rightarrow \infty}\left[M_{\infty}^{4}\right]=\lim _{\xi_{1}, \xi_{2} \rightarrow \infty}\left[\frac{\xi_{1}}{1-\xi_{1}}\right]=-1 \\
\lim _{\xi_{1}, \xi_{2} \rightarrow \infty}\left[M_{\infty}^{5}\right]=\lim _{\xi_{1}, \xi_{2} \rightarrow \infty}\left[\frac{\xi_{2}}{1-\xi_{2}}\right]=-1 \\
\lim _{\xi_{1}, \xi_{2} \rightarrow \infty}\left[M_{\infty}^{3}\right]=\lim _{\xi_{1}, \xi_{2} \rightarrow \infty}\left[1-\frac{\xi_{1}}{1-\xi_{1}}-\frac{\xi_{2}}{1-\xi_{2}}\right]=3
\end{gathered}
$$

Portanto, as coordenadas globais do pólo podem ser determinadas empregando a seguinte equação:

$$
x_{i}^{p}=x_{i}^{3}-\left(x_{i}^{4}-x_{i}^{3}\right)-\left(x_{i}^{5}-x_{i}^{3}\right)
$$

$\mathrm{ou}$

$$
x_{i}^{p}=x_{i}^{3}-x_{i}^{43}-x_{i}^{53}
$$

Conforme demonstrado na expressão 4.99, no caso das duas direções mapeadas o pólo é único. Desta forma, os ECI deste tipo devem ser gerados de forma a aproximar este pólo do centro de decaimento do problema.

\subsubsection{Cálculo do Jacobiano}

Finalmente, após todas estas análises, chega-se à parte mais importante desta Seção que é a determinação do Jacobiano da transformação do sistema de coordenadas globais para o sistema homogêneo local. O Jacobiano é dado por:

$$
\left.\right|^{\infty} J|=| \begin{array}{ll}
\frac{\partial x_{1}}{\partial \xi_{1}} & \frac{\partial x_{2}}{\partial \xi_{1}} \\
\frac{\partial x_{1}}{\partial \xi_{2}} & \frac{\partial x_{2}}{\partial \xi_{2}}
\end{array} \mid=\frac{\partial x_{1}}{\partial \xi_{1}} \frac{\partial x_{2}}{\partial \xi_{2}}-\frac{\partial x_{2}}{\partial \xi_{1}} \frac{\partial x_{1}}{\partial \xi_{2}}
$$

O símbolo " $\infty$ " indica que o Jacobiano se refere a um ECI. Considerando inicialmente 
decaimento somente na direção $\xi_{1}$, efetua-se o seguinte desenvolvimento:

$$
\begin{aligned}
\frac{\partial x_{1}}{\partial \xi_{1}} & =\frac{\partial M_{\infty 1}^{4}}{\partial \xi_{1}} x_{1}^{4}+\frac{\partial M_{\infty 1}^{2}}{\partial \xi_{1}} x_{1}^{2}+\frac{\partial M_{\infty 1}^{3}}{\partial \xi_{1}} x_{1}^{3}=\frac{x_{1}^{4}-x_{1}^{3}}{\left(1-\xi_{1}\right)^{2}} \\
\frac{\partial x_{2}}{\partial \xi_{1}} & =\frac{\partial M_{\infty 1}^{4}}{\partial \xi_{1}} x_{2}^{4}+\frac{\partial M_{\infty 1}^{2}}{\partial \xi_{1}} x_{2}^{2}+\frac{\partial M_{\infty 1}^{3}}{\partial \xi_{1}} x_{2}^{3}=\frac{x_{2}^{4}-x_{2}^{3}}{\left(1-\xi_{1}\right)^{2}} \\
\frac{\partial x_{1}}{\partial \xi_{2}} & =\frac{\partial M_{\infty 1}^{4}}{\partial \xi_{2}} x_{1}^{4}+\frac{\partial M_{\infty 1}^{2}}{\partial \xi_{2}} x_{1}^{2}+\frac{\partial M_{\infty 1}^{3}}{\partial \xi_{2}} x_{1}^{3}=x_{1}^{2}-x_{1}^{3} \\
\frac{\partial x_{2}}{\partial \xi_{2}} & =\frac{\partial M_{\infty 1}^{4}}{\partial \xi_{2}} x_{2}^{4}+\frac{\partial M_{\infty 1}^{2}}{\partial \xi_{2}} x_{2}^{2}+\frac{\partial M_{\infty 1}^{3}}{\partial \xi_{2}} x_{2}^{3}=x_{2}^{2}-x_{2}^{3}
\end{aligned}
$$

Substituindo as igualdades 4.101, 4.102, 4.103 e 4.104 na expressão 4.100, obtém-se:

$$
\left.\right|^{\infty} J \mid=\frac{\left(x_{1}^{4}-x_{1}^{3}\right)\left(x_{2}^{4}-x_{2}^{3}\right)-\left(x_{1}^{2}-x_{1}^{3}\right)\left(x_{2}^{2}-x_{2}^{3}\right)}{\left(1-\xi_{1}\right)^{2}}=\frac{2 A}{\left(1-\xi_{1}\right)^{2}}
$$

sendo $A$ a área do triângulo definido pelos nós 4,2 e 3 do ECI.

Na sequência, considera-se a direção $\xi_{2}$ mapeada:

$$
\begin{gathered}
\frac{\partial x_{1}}{\partial \xi_{1}}=\frac{\partial M_{\infty 2}^{1}}{\partial \xi_{1}} x_{1}^{1}+\frac{\partial M_{\infty 2}^{5}}{\partial \xi_{1}} x_{1}^{5}+\frac{\partial M_{\infty 2}^{3}}{\partial \xi_{1}} x_{1}^{3}=x_{1}^{1}-x_{1}^{3} \\
\frac{\partial x_{2}}{\partial \xi_{1}}=\frac{\partial M_{\infty 2}^{1}}{\partial \xi_{1}} x_{2}^{1}+\frac{\partial M_{\infty 2}^{5}}{\partial \xi_{1}} x_{2}^{5}+\frac{\partial M_{\infty 2}^{3}}{\partial \xi_{1}} x_{2}^{3}=x_{2}^{1}-x_{2}^{3} \\
\frac{\partial x_{1}}{\partial \xi_{2}}=\frac{\partial M_{\infty 2}^{1}}{\partial \xi_{2}} x_{1}^{1}+\frac{\partial M_{\infty 2}^{5}}{\partial \xi_{2}} x_{1}^{5}+\frac{\partial M_{\infty 2}^{3}}{\partial \xi_{2}} x_{1}^{3}=\frac{x_{1}^{5}-x_{1}^{3}}{\left(1-\xi_{2}\right)^{2}} \\
\frac{\partial x_{2}}{\partial \xi_{2}}=\frac{\partial M_{\infty 2}^{1}}{\partial \xi_{2}} x_{2}^{1}+\frac{\partial M_{\infty 2}^{5}}{\partial \xi_{2}} x_{2}^{5}+\frac{\partial M_{\infty 2}^{3}}{\partial \xi_{2}} x_{2}^{3}=\frac{x_{2}^{5}-x_{2}^{3}}{\left(1-\xi_{2}\right)^{2}}
\end{gathered}
$$

O Jacobiano é então dado por:

$$
\left.\right|^{\infty} J \mid=\frac{\left(x_{1}^{1}-x_{1}^{3}\right)\left(x_{2}^{1}-x_{2}^{3}\right)-\left(x_{1}^{5}-x_{1}^{3}\right)\left(x_{2}^{5}-x_{2}^{3}\right)}{\left(1-\xi_{2}\right)^{2}}=\frac{2 A}{\left(1-\xi_{2}\right)^{2}}
$$

e neste caso $A$ é a área do triângulo definido pelos nós 1, 5 e 3 do ECI.

Por fim, considera-se o Jacobiano quando as duas direções são mapeadas:

$$
\frac{\partial x_{1}}{\partial \xi_{1}}=\frac{\partial M_{\infty}^{4}}{\partial \xi_{1}} x_{1}^{4}+\frac{\partial M_{\infty}^{5}}{\partial \xi_{1}} x_{1}^{5}+\frac{\partial M_{\infty}^{3}}{\partial \xi_{1}} x_{1}^{3}=\frac{x_{1}^{4}-x_{1}^{3}}{\left(1-\xi_{1}\right)^{2}}
$$




$$
\begin{aligned}
& \frac{\partial x_{2}}{\partial \xi_{1}}=\frac{\partial M_{\infty}^{4}}{\partial \xi_{1}} x_{2}^{4}+\frac{\partial M_{\infty}^{5}}{\partial \xi_{1}} x_{2}^{5}+\frac{\partial M_{\infty}^{3}}{\partial \xi_{1}} x_{2}^{3}=\frac{x_{2}^{4}-x_{2}^{3}}{\left(1-\xi_{1}\right)^{2}} \\
& \frac{\partial x_{1}}{\partial \xi_{2}}=\frac{\partial M_{\infty}^{4}}{\partial \xi_{2}} x_{1}^{4}+\frac{\partial M_{\infty}^{5}}{\partial \xi_{2}} x_{1}^{5}+\frac{\partial M_{\infty}^{3}}{\partial \xi_{2}} x_{1}^{3}=\frac{x_{1}^{5}-x_{1}^{3}}{\left(1-\xi_{2}\right)^{2}} \\
& \frac{\partial x_{2}}{\partial \xi_{2}}=\frac{\partial M_{\infty}^{4}}{\partial \xi_{2}} x_{2}^{4}+\frac{\partial M_{\infty}^{5}}{\partial \xi_{2}} x_{2}^{5}+\frac{\partial M_{\infty}^{3}}{\partial \xi_{2}} x_{2}^{3}=\frac{x_{2}^{5}-x_{2}^{3}}{\left(1-\xi_{2}\right)^{2}}
\end{aligned}
$$

sendo o Jacobiano dado por:

$$
\left.\right|^{\infty} J \mid=\frac{\left(x_{1}^{4}-x_{1}^{3}\right)\left(x_{2}^{4}-x_{2}^{3}\right)-\left(x_{1}^{5}-x_{1}^{3}\right)\left(x_{2}^{5}-x_{2}^{3}\right)}{\left(1-\xi_{1}\right)^{2}\left(1-\xi_{2}\right)^{2}}=\frac{2 A}{\left(1-\xi_{1}\right)^{2}\left(1-\xi_{2}\right)^{2}}
$$

Neste caso, $A$ é a área do triângulo definido pelos nós 4, 5 e 3 do ECI.

\subsubsection{Inclusão dos ECIs no equacionamento}

Depois de definidos os Jacobianos, torna-se possível incluir nas equações escritas para o contorno a influência dos ECIs. Desta forma, a expressão 2.103 se torna:

$$
\begin{gathered}
\mathbf{c}^{i} \mathbf{u}^{i}+\sum_{j=1}^{n e}\left[\left(\int_{\Gamma_{j}} \mathbf{p}^{*} \boldsymbol{\Phi} d \Gamma\right) \mathbf{u}^{j}\right]+\sum_{j=1}^{n e c i}\left[\left(\int_{\Gamma_{j}^{\infty}} \mathbf{p}^{*} \boldsymbol{\Phi} d \Gamma^{\infty}\right) \mathbf{u}^{j}\right] \\
=\sum_{j=1}^{n e}\left[\left(\int_{\Gamma_{j}} \mathbf{u}^{*} \boldsymbol{\Phi} d \Gamma\right) \mathbf{p}^{j}\right]+\sum_{j=1}^{n e c i}\left[\left(\int_{\Gamma_{j}^{\infty}} \mathbf{u}^{*} \boldsymbol{\Phi} d \Gamma^{\infty}\right) \mathbf{p}^{j}\right]
\end{gathered}
$$

Cada lado da igualdade é dividido em dois somatórios, um para os ECs da malha e outro para os ECIs. A parte referente aos ECs pode ser tratada de forma idêntica à apresentada no capítulo 2, mas as integrais sobre os ECIs ocorrem sobre superfícies infinitas. Isto é indicado na equação pelo símbolo " $\infty$ ”. Para transformar do sistema de coordenadas global para o local, emprega-se o Jacobiano deduzido no Capítulo 2 para os ECs e os Jacobianos determinados nesta seção para os ECIs. O resultado é a seguinte expressão:

$$
\begin{gathered}
\mathbf{c}^{i} \mathbf{u}^{i}+\sum_{j=1}^{n e}\left[\left(\int_{\gamma} \mathbf{p}^{*} \boldsymbol{\Phi}|\mathbf{J}|^{j} d \xi_{1} d \xi_{2}\right) \mathbf{u}^{j}\right]+\sum_{j=1}^{n e c i}\left[\left(\left.\left.\int_{\gamma} \mathbf{p}^{*} \boldsymbol{\Phi}\right|^{\infty} \mathbf{J}\right|^{j} d \xi_{1} d \xi_{2}\right) \mathbf{u}^{j}\right] \\
=\sum_{j=1}^{n e}\left[\left(\int_{\gamma} \mathbf{u}^{*} \boldsymbol{\Phi}|\mathbf{J}|^{j} d \xi_{1} d \xi_{2}\right) \mathbf{p}^{j}\right]+\sum_{j=1}^{n e c i}\left[\left(\left.\left.\int_{\gamma} \mathbf{u}^{*} \boldsymbol{\Phi}\right|^{\infty} \mathbf{J}\right|^{j} d \xi_{1} d \xi_{2}\right) \mathbf{p}^{j}\right]
\end{gathered}
$$

Neste trabalho, as integrais não singulares da equação 4.117 são calculadas numerica- 
mente empregando a mesma técnica utilizada no Capítulo 2. Assim, escreve-se:

$$
\begin{gathered}
\mathbf{c}^{i} \mathbf{u}^{i}+\sum_{j=1}^{n e}\left[|\mathbf{J}|^{j}\left\{\sum_{k=1}^{n h}\left(\mathbf{w}_{k} \mathbf{p}_{k}^{*} \boldsymbol{\Phi}_{k}\right)\right\} \mathbf{u}^{j}\right]+\sum_{j=1}^{n e c i}\left[\left\{\sum_{k=1}^{n h}\left(\left.\left.\mathbf{w}_{k} \mathbf{p}_{k}^{*} \boldsymbol{\Phi}_{k}\right|^{\infty} \mathbf{J}\right|_{k} ^{j}\right)\right\} \mathbf{u}^{j}\right] \\
=\sum_{j=1}^{n e}\left[|\mathbf{J}|^{j}\left\{\sum_{k=1}^{n h}\left(\mathbf{w}_{k} \mathbf{u}_{k}^{*} \boldsymbol{\Phi}_{k}\right)\right\} \mathbf{p}^{j}\right]+\sum_{j=1}^{n e c i}\left[\left\{\sum_{k=1}^{n h}\left(\left.\left.\mathbf{w}_{k} \mathbf{u}_{k}^{*} \boldsymbol{\Phi}_{k}\right|^{\infty} \mathbf{J}\right|_{k} ^{j}\right)\right\} \mathbf{p}^{j}\right]
\end{gathered}
$$

Assim como descrito no Capítulo 2, o termo nh se refere ao número de pontos de integração definidos sobre a superfície de cada elemento e as coordenadas e pesos dos pontos de Hammer podem ser encontrados tabelados na literatura. A única diferença da integral sobre os ECIs é que o Jacobiano também é função das coordenadas adimensionais, portanto não pode ser excluído do somatório de pontos de Hammer. Assim, calcula-se o valor do Jacobiando dos ECIs ponto a ponto.

\subsubsection{Tratamento da singularidade nos ECIs}

Caso o ponto fonte esteja localizado em um dos vértices de um EC ou ECI a ser integrado, as integrais 4.117 apresentam problemas de singularidade. Desta forma, a técnica de integração numérica apresentada anteriormente pode causar imprecisões nos resultados. Este problema foi resolvido para o caso dos ECs singulares no Capítulo 2, no qual é empregada a técnica de Guiggiani e Gigante (1990) para o cálculo destas integrais semi-analiticamente. Neste trabalho esta mesma técnica é utilizada para os ECIs, mas neste caso as integrais singulares são calculadas de forma totalmente numérica. É dado maior destaque às partes da dedução que são distintas daquela apresentada na Seção 2.5.2, e as partes semelhantes são resumidas.

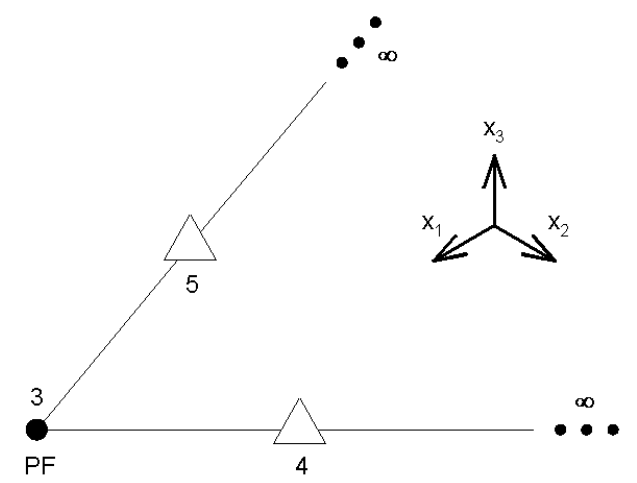

Figura 4.9: ECI no sistema de coordenadas globais

Considera-se um ECI a ser integrado e que o ponto fonte esteja posicionado em um 
dos seus vértices, assim como apresentado na figura 4.9. Para facilitar o desenvolvimento das expressões, extrai-se da equação 4.117 as parcelas singulares. Estas parcelas são:

$$
\begin{aligned}
& \mathbf{I}_{1}=\left.\int_{\gamma_{P}} \mathbf{p}^{*} \boldsymbol{\Phi}\right|^{\infty} \mathbf{J} \mid d \xi_{1} d \xi_{2} \\
& \mathbf{I}_{2}=\left.\int_{\gamma_{P}} \mathbf{u}^{*} \boldsymbol{\Phi}\right|^{\infty} \mathbf{J} \mid d \xi_{1} d \xi_{2}
\end{aligned}
$$

Nas integrais 4.119 e 4.120 , a letra $P$ subscrita indica que o ponto fonte pertence à superfície a ser integrada. Na figura 4.9 o ponto fonte está localizado no nó 3 do ECI, na numeração local. Apesar de estar representado o caso em que as duas direções são mapeadas, as deduções aqui desenvolvidas são válidas também para os outros casos. Nota-se que o ECI da figura está no sistema de coordenadas globais, por isso seus extremos estão no infinito. Na sequência deve-se representá-lo no sistema local oblíquo, assim como ilustrado na figura 4.10 .

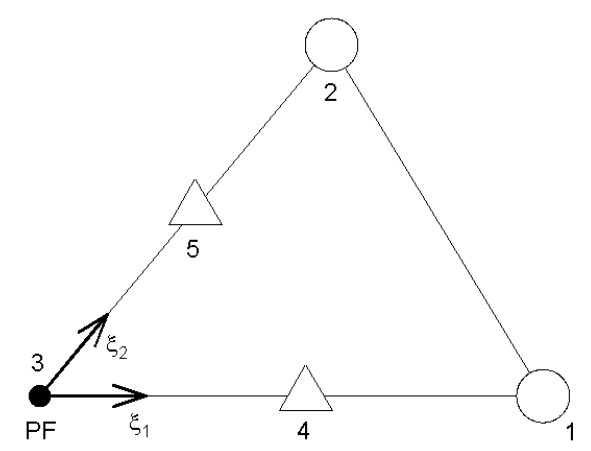

Figura 4.10: ECI no sistema de coordenadas locais oblíquas

No caso desta segunda figura, devido ao mapeamento, os nós 1 e 2 estão posicionados em coordenadas finitas. A passagem do sistema de coordenadas retangular global ao oblícuo local se dá por meio das equações 4.82, 4.83 e 4.84, conforme descrito anteriormente. Os Jacobianos necessários para esta transformação foram deduzidos na Seção 4.5.4. sendo representados em 4.119 e 4.120 pelo termo $\left.\right|^{\infty} \mathbf{J} \mid$.

Seguindo com a dedução, o passo seguinte é passar do sistema de coordenadas local oblíquo $\xi_{1} \xi_{2}$ para o sistema local retangular $\bar{x}_{1} \bar{x}_{2} \bar{x}_{3}$. O sistema $\bar{x}_{1} \bar{x}_{2} \bar{x}_{3}$ considerado na Seção 2.5 .2 é igual ao sistema retangular global exceto por uma rotação e translação de eixos, de forma que o segundo Jacobiano cancela o primeiro. Neste trabalho, isto não ocorre no caso dos ECIs. Foi verificado que é possível criar um sistema retangular 
local para o qual os nós 1 e 2 não estejam posicionados no infinito. É importante que isto aconteça para que seja possível, posteriormente, calcular as coordenadas de pontos de integração posicionados ao longo do segmento de reta entre os nós 1 e 2 do ECI. Conforme foi apresentado na Seção 2.5.2, o Jacobiano da transformação de $\xi_{1} \xi_{2}$ para $\bar{x}_{1} \bar{x}_{2} \bar{x}_{3}$ é:

$$
\left|\mathbf{J}_{1}\right|=\frac{1}{2 A}
$$

sendo $A$ a área do elemento no sistema $\bar{x}_{1} \bar{x}_{2} \bar{x}_{3}$. Isto significa que não é obrigatório que $A$ seja a área do elemento no sistema global, ele pode resultar com quaisquer dimensões desejadas desde que o Jacobiano $\left|\mathbf{J}_{\mathbf{1}}\right|$ seja considerado. A figura 4.11 ilustra o elemento no sistema retangular local.

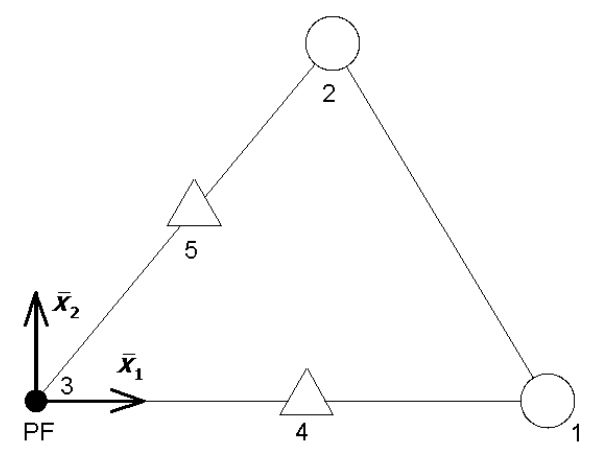

Figura 4.11: ECI no sistema de coordenadas retangular local

Desta forma, para tornar mais simples as expressões, optou-se por transformar o ECI de forma que o Jacobiano $\left|\mathbf{J}_{1}\right|$ elimine o numerador do Jacobiano $\left.\right|^{\infty} \mathbf{J} \mid$. Portanto:

$$
\left.\right|^{\infty} \mathbf{J}|| \mathbf{J}_{1} \mid=\frac{2 A}{f\left(\xi_{1}, \xi_{2}\right)} \frac{1}{2 A}=\frac{1}{f\left(\xi_{1}, \xi_{2}\right)}
$$

A função $f\left(\xi_{1}, \xi_{2}\right)$ depende da direção mapeada, e a área $A$ corresponde ao triângulo formado pelos nós do ECI de coordenadas finitas e os nós auxiliares do mapeamento. Para o caso da figura 4.11, $f\left(\xi_{1}, \xi_{2}\right)$ é o denominador da expressão 4.115 e $A$ é a área do triângulo de vértices 3,4 e 5 .

Portanto, após a transformação para o sistema $\bar{x}_{1} \bar{x}_{2} \bar{x}_{3}$, as integrais se tornam:

$$
\mathbf{I}_{1}=\left.\int_{\overline{\bar{\Gamma}}_{P}} \mathbf{p}^{*} \boldsymbol{\Phi}\right|^{\infty} \mathbf{J}|| \mathbf{J}_{1} \mid d \bar{x}_{1} d \bar{x}_{2}
$$




$$
\mathbf{I}_{2}=\left.\int_{\bar{\Gamma}_{P}} \mathbf{u}^{*} \boldsymbol{\Phi}\right|^{\infty} \mathbf{J}|| \mathbf{J}_{1} \mid d \bar{x}_{1} d \bar{x}_{2}
$$

A fase seguinte é passar do sistema $\bar{x}_{1} \bar{x}_{2} \bar{x}_{3}$ para o sistema polar $r \theta$, conforme ilustrado na figura 4.12. O Jacobiano desta transformação foi deduzido na Seção 2.5.2, e é:

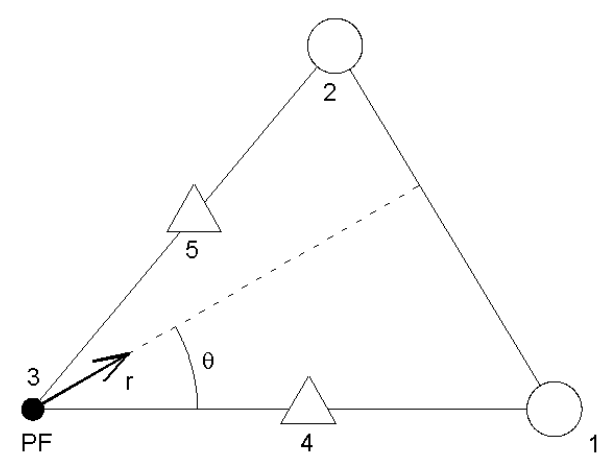

Figura 4.12: ECI no sistema de coordenadas polares

$$
\left|\mathbf{J}_{2}\right|=r
$$

Assim, substituindo o Jacobiano $\left|\mathbf{J}_{2}\right|$ nas integrais, obtém-se:

$$
\begin{aligned}
& \mathbf{I}_{1}=\left.\int_{0}^{2 \pi} \int_{0}^{R} \mathbf{p}^{*} \boldsymbol{\Phi}\right|^{\infty} \mathbf{J}|| \mathbf{J}_{1}|| \mathbf{J}_{2} \mid d r d \theta \\
& \mathbf{I}_{2}=\left.\int_{0}^{2 \pi} \int_{0}^{R} \mathbf{u}^{*} \boldsymbol{\Phi}\right|^{\infty} \mathbf{J}|| \mathbf{J}_{1}|| \mathbf{J}_{2} \mid d r d \theta
\end{aligned}
$$

sendo $R$ a distância do ponto fonte ao perímetro do ECI. Neste ponto surge uma diferença relevante da dedução desenvolvida na Seção 2.5.2. Neste caso a variárel $r$ não é integrada analiticamente porque a presença do Jacobiano $\left.\right|^{\infty} \mathbf{J} \mid$, que também é função de $\xi_{1}$ e $\xi_{2}$, causa complicações neste procedimento. A solução encontrada foi integrar $r$ numericamente, assim como $\theta$. Segundo Guiggiani e Gigante (1990), como a singularidade é eliminada, é possível obter resultados precisos mesmo com integrais numéricas. Será visto ao final do Capítulo, por meio da resolução de exemplos, que esta formulação apresenta precisão satisfatória.

Dando-se sequência à dedução, deve-se transformar a coordenada $\theta$ para uma coordenada $\hat{\Gamma}$, conforme apresentado na figura 4.13. Na Seção 2.5.2 foi visto que o Jacobiano desta transformação é:

$$
\left|\mathbf{J}_{3}\right|=\frac{1}{R} \frac{d R}{d \hat{\eta}}
$$




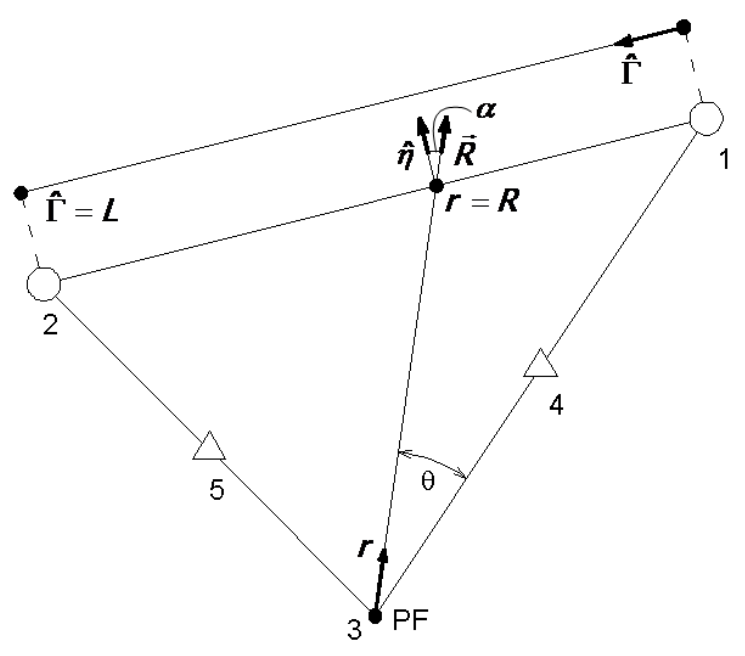

Figura 4.13: ECI no sistema de coordenadas $r \hat{\Gamma}$

A distância do ponto fonte ao perímetro do triângulo, no sistema $\bar{x}_{1} \bar{x}_{2} \bar{x}_{3}$, é $R$. A coordenada $\hat{\Gamma}$ varia de zero a $L$, que é o comprimento do segmento de reta no mesmo sistema. O versor $\vec{R}$ indica a direção e sentido de $R$ e o versor $\hat{\eta}$ é perpendicular ao segmento de reta. A derivada de $R$ em relação a $\hat{\eta}$, necessária para o cálculo do Jacobiano $\left|\mathbf{J}_{\mathbf{3}}\right|$, corresponde ao co-seno do ângulo $\alpha$ indicado na figura 4.13 , e é calculada conforme apresentado na Seção 2.5.2. Assim, substituindo-se o Jacobiano nas integrais, obtém-se:

$$
\begin{aligned}
& \mathbf{I}_{1}=\left.\int_{0}^{L} \int_{0}^{R} \mathbf{p}^{*} \boldsymbol{\Phi}\right|^{\infty} \mathbf{J}|| \mathbf{J}_{1}|| \mathbf{J}_{2}|| \mathbf{J}_{3} \mid d r d \hat{\Gamma} \\
& \mathbf{I}_{2}=\left.\int_{0}^{L} \int_{0}^{R} \mathbf{u}^{*} \boldsymbol{\Phi}\right|^{\infty} \mathbf{J}|| \mathbf{J}_{1}|| \mathbf{J}_{2}|| \mathbf{J}_{3} \mid d r d \hat{\Gamma}
\end{aligned}
$$

A transformação final que antecede a integração numérica é dos limites de integração para os extremos -1 e +1 , para que sejam empregados pontos de Gauss posteriormente. Isto é feito transformando $r$ para uma coordenada adimensional $\varsigma$ e $\hat{\Gamma}$ para uma coordenada adimensional $\zeta$, assim como ilustrado na figura 4.14. Os seguintes Jacobianos dever ser considerados:

$$
\begin{aligned}
& \left|\mathbf{J}_{4}\right|=\frac{L}{2} \\
& \left|\mathbf{J}_{5}\right|=\frac{R}{2}
\end{aligned}
$$

Desta forma, as expressões finais a serem integradas são:

$$
\mathbf{I}_{1}=\left.\int_{-1}^{+1} \int_{-1}^{+1} \mathbf{p}^{*} \boldsymbol{\Phi}\right|^{\infty} \mathbf{J}|| \mathbf{J}_{1}|| \mathbf{J}_{2}|| \mathbf{J}_{3}|| \mathbf{J}_{4}|| \mathbf{J}_{5} \mid d \varsigma d \zeta
$$




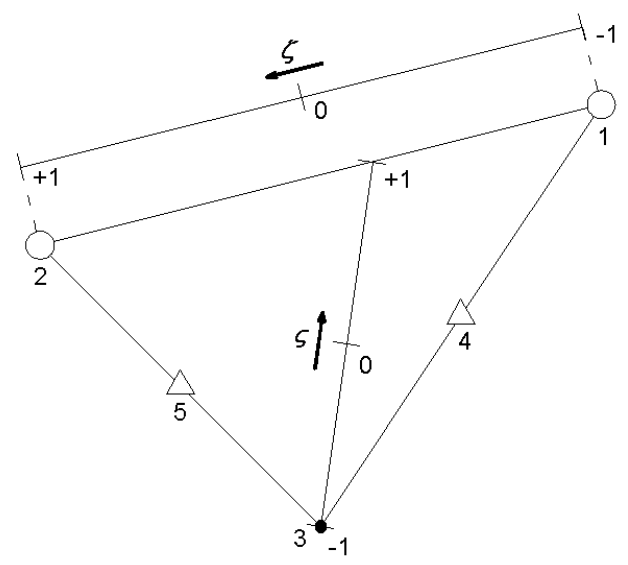

Figura 4.14: ECI no sistema de coordenadas $\varsigma \zeta$

$$
\mathbf{I}_{2}=\left.\int_{-1}^{+1} \int_{-1}^{+1} \mathbf{u}^{*} \boldsymbol{\Phi}\right|^{\infty} \mathbf{J}|| \mathbf{J}_{1}|| \mathbf{J}_{2}|| \mathbf{J}_{3}|| \mathbf{J}_{4}|| \mathbf{J}_{5} \mid d \varsigma d \zeta
$$

A seguir, as expressões dentro das integrais são trabalhadas de forma mais explícita. Inicia-se escrevendo as soluções fundamentais deduzidas no Capítulo 2 ;

$$
\begin{gathered}
u_{l k}^{*}=\frac{(3-4 \nu) \delta_{l k}+r_{, l} r_{, k}}{16 \pi \mu(1-\nu) r} \\
p_{l k}^{*}=\frac{(2 \nu-1)\left(\eta_{l} r_{, k}-\eta_{k} r_{, l}\right)}{8 \pi(1-\nu) r^{2}}
\end{gathered}
$$

Observa-se que é possível combinar estas expressões com o Jacobiano $\left|\mathbf{J}_{\mathbf{2}}\right|$, previamente definido. Assim:

$$
\begin{gathered}
u_{l k}^{*}\left|\mathbf{J}_{2}\right|=\frac{(3-4 \nu) \delta_{l k}+r_{, l} r_{, k}}{16 \pi \mu(1-\nu) r} r=\frac{(3-4 \nu) \delta_{l k}+r_{, l} r_{, k}}{16 \pi \mu(1-\nu)} \\
p_{l k}^{*}\left|\mathbf{J}_{2}\right|=\frac{(2 \nu-1)\left(\eta_{l} r_{, k}-\eta_{k} r_{, l}\right)}{8 \pi(1-\nu) r^{2}} r=\frac{(2 \nu-1)\left(\eta_{l} r_{, k}-\eta_{k} r_{, l}\right)}{8 \pi(1-\nu) r}
\end{gathered}
$$

Além disto, foi demonstrado anteriormente que:

$$
\left.\right|^{\infty} \mathbf{J}|| \mathbf{J}_{1} \mid=\frac{1}{f\left(\xi_{1}, \xi_{2}\right)}
$$

sendo que, para decaimento na direção $\xi_{1}$ :

$$
f\left(\xi_{1}, \xi_{2}\right)=\left(1-\xi_{1}\right)^{2}
$$


para decaimento na direção $\xi_{2}$ :

$$
f\left(\xi_{1}, \xi_{2}\right)=\left(1-\xi_{2}\right)^{2}
$$

e para decaimento nas duas direções:

$$
f\left(\xi_{1}, \xi_{2}\right)=\left(1-\xi_{1}\right)^{2}\left(1-\xi_{2}\right)^{2}
$$

Considerando que a contribuição é de uma função de forma $i$, torna-se possível escrever:

$$
\Phi=\xi_{i}
$$

Combinando este termo com o anterior, obtém-se:

$$
\left.\right|^{\infty} \mathbf{J}|| \mathbf{J}_{1} \mid \mathbf{\Phi}=\frac{\xi_{i}}{f\left(\xi_{1}, \xi_{2}\right)}
$$

Os demais termos a serem multiplicados são considerados abaixo:

$$
\left|\mathbf{J}_{3}\right|\left|\mathbf{J}_{4}\right|\left|\mathbf{J}_{5}\right|=\frac{1}{R} \frac{\partial R}{\partial \hat{\eta}} \frac{R}{2} \frac{L}{2}=\frac{\partial R}{\partial \hat{\eta}} \frac{L}{4}
$$

Combinando estes termos com os anteriores, conclui-se que as integrais a serem calculadas são:

$$
\begin{gathered}
I_{l k i 1}=\int_{-1}^{+1} \int_{-1}^{+1} \frac{(3-4 \nu) \delta_{l k}+r_{, l} r_{, k}}{16 \pi \mu(1-\nu)} \frac{\xi_{i}}{f\left(\xi_{1}, \xi_{2}\right)} \frac{\partial R}{\partial \hat{\eta}} \frac{L}{4} d \varsigma d \zeta \\
I_{l k i 2}=\int_{-1}^{+1} \int_{-1}^{+1} \frac{(2 \nu-1)\left(\eta_{l} r_{, k}-\eta_{k} r_{, l}\right)}{8 \pi(1-\nu) r} \frac{\xi_{i}}{f\left(\xi_{1}, \xi_{2}\right)} \frac{\partial R}{\partial \hat{\eta}} \frac{L}{4} d \varsigma d \zeta
\end{gathered}
$$

Estas integrais são calculadas numericamente utilizando pontos de Gauss. Desta forma, escreve-se:

$$
\begin{gathered}
I_{l k i 1}=\sum_{g \zeta=1}^{n g \zeta}\left\{\sum_{g \varsigma=1}^{n g \varsigma}\left[\left(\frac{(3-4 \nu) \delta_{l k}+r_{, l} r_{, k}}{16 \pi \mu(1-\nu)} \frac{\xi_{i}}{f\left(\xi_{1}, \xi_{2}\right)} \frac{\partial R}{\partial \hat{\eta}} \frac{L}{4}\right) w_{g \varsigma}\right] w_{g \zeta}\right\} \\
I_{l k i 2}=\sum_{g \zeta=1}^{n g \zeta}\left\{\sum_{g \varsigma=1}^{n g \varsigma}\left[\left(\frac{(2 \nu-1)\left(\eta_{l} r_{, k}-\eta_{k} r_{, l}\right)}{8 \pi(1-\nu) r} \frac{\xi_{i}}{f\left(\xi_{1}, \xi_{2}\right)} \frac{\partial R}{\partial \hat{\eta}} \frac{L}{4}\right) w_{g \varsigma}\right] w_{g \zeta}\right\}
\end{gathered}
$$

Aparecem dois somatórios nas igualdades, um para cada direção dos pontos de Gauss. Para a direção ऽ o número de pontos de Gauss é definido como ngs e o peso do ponto é 
indicado como $w_{g \varsigma}$. Na direção $\zeta$, o número de pontos é $n g \zeta$ seus pesos $w_{g \zeta}$. Para tornar mais prático o cálculo destas expressões, apresenta-se a seguir um roteiro:

1. Determina-se as coordenadas dos vértices do ECI e do ponto fonte no sistema retangular local $\bar{x}_{1} \bar{x}_{2} \bar{x}_{3}$

2. Calcula-se $L$ e as componentes do versor $\hat{\eta}$;

3. Dado um ponto de Gauss $g \zeta$, na direção $\zeta$, calcula-se suas coordenadas no sistema retangular local $\bar{x}_{1} \bar{x}_{2} \bar{x}_{3}$ e no sistema global a partir das coordenadas dos vértices do ECI. Emprega-se as expressões 2.182 e 2.183, apresentadas na Seção 2.5.2.

4. Determina-se $R$ e $\frac{\partial R}{\partial \hat{\eta}}$;

5. Calcula-se para o ponto de Gauss $g \varsigma$, na direção $\varsigma$, as coordenadas do ponto campo no sistema retangular local $\bar{x}_{1} \bar{x}_{2} \bar{x}_{3}$ a partir das coordenadas do ponto fonte e do ponto de Gauss na direção $\zeta$. Para isto, emprega-se novamente a expressão 2.183

6. Determina-se as coordenadas do ponto campo no sistema local oblíquo, empregando a expressão 2.120

7. A partir das coordenadas do ponto campo, calcula-se a expressão 4.144:

8. Calcula-se as coordenadas do ponto campo no sistema global a partir de suas coordenadas homogêneas e das funções de mapeamento $4.82,4.83$ ou 4.84 .

9. Calcula-se $r$ e suas derivadas;

10. Como os demais termos são conhecidos, calcula-se o valor das expressões 4.148 e 4.149 para o ponto campo considerado.

\subsection{Exemplos}

O objetivo dos exemplos apresentados nesta Seção é demosntrar a eficiência e precisão da formulação de ECIs descrita neste Capítulo. Nos exemplos, a distância a partir da qual os ECIs são empregados é determinada em um estudo realizado utilizando malhas pouco refinadas, no qual verifica-se a partir de qual distância o resultado não é mais alterado de forma significativa. Depois de encontrada e fixada tal distância, parte-se para um segundo 
estudo no qual é variado somente o refinamento da malha. O refinamento final é então determinado quando verifica-se que um aumento no número de graus de liberdade não altera os resultados de forma significativa, consistindo na convergência para o valor final a ser apresentado no exemplo em questão.

\subsubsection{Semi-espaço infinito homogêneo}

O problema a ser considerado é apresentado na figura 4.15. É aplicado, na superfície de um semi-espaço infinito homogêneo, um carregamento quadrado de $100 \mathrm{kN} / \mathrm{m}^{2}$ e com $2 m$ de aresta. O material tem módulo de elasticidade igual a $10000 \mathrm{kN} / \mathrm{m}^{2}$ e coeficiente de Poisson igual a zero. O ponto $A$ está posicionado no centro do quadrado e o ponto $B$ se encontra em um dos seus vértices.

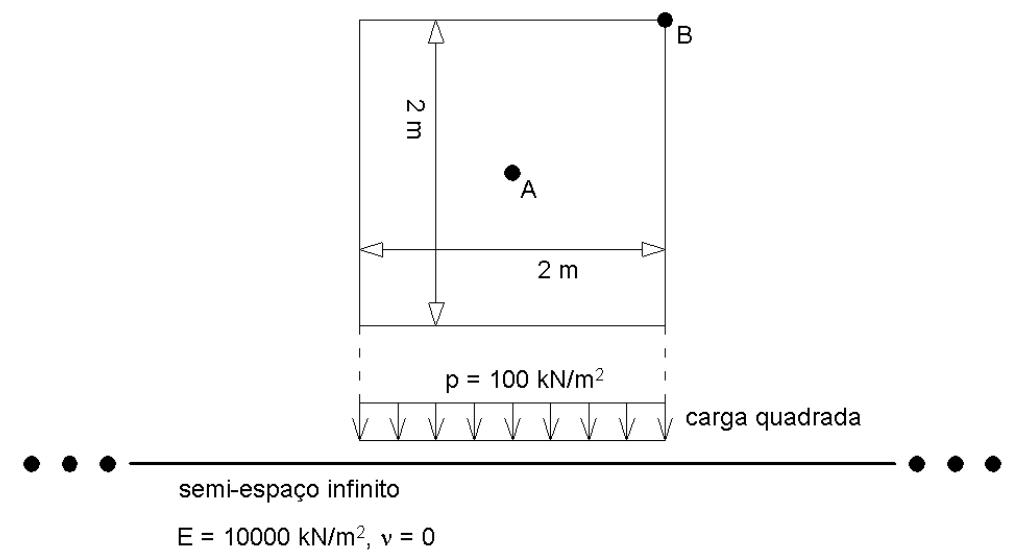

Figura 4.15: Semi-espaço infinito homogêneo com carregamento quadrado

Para simular este problema foi criada uma malha com 73 nós, 128 ECs e 32 ECIs, conforme ilustrado na figura 4.16. O quadrado destacado no centro corresponde à área carregada, as linhas tracejadas representam os ECIs e os pontos destacados nas bordas da malha de ECs são os que recebem as contribuições dos ECIs. É importante notar que os ECIs não adicionam novos graus de liberdade à malha original, não alterando as dimensões do sistema de equações final. Esta vantagem nem sempre ocorre nas formulações presentes na literatura, como será visto mais adiante.

Na tabela 4.1 são apresentados os deslocamentos verticais calculados para os pontos $A$ e $B$, indicados na figura 4.15 .

É apresentada, primeiramente, a solução exata do deslocamento vertical nos pontos $A$ e $B$. Estes valores são empregados como referência para o cálculo do erro nas demais 


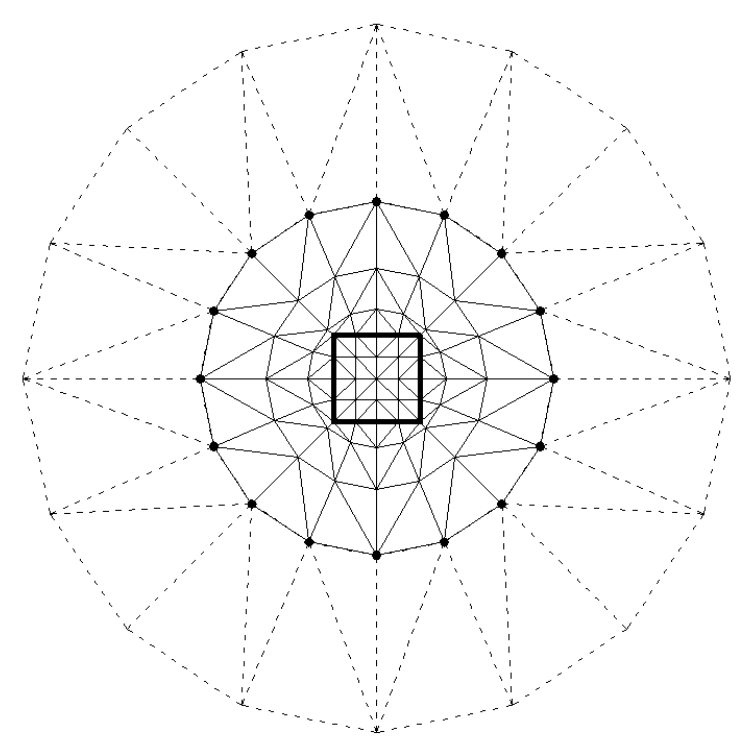

Figura 4.16: Malha de EC/ECI empregada

\begin{tabular}{|c|c|c|c|c|}
\cline { 2 - 5 } \multicolumn{1}{c|}{} & Ponto $A$ & Erro (\%) & Ponto $B$ & Erro (\%) \\
\hline Solução exata & 2,2444 & - & 1,1222 & \\
\hline Moser et al. (2004) & 2,2520 & 0,7 & 1,1298 & 0,4 \\
\hline Sem ECIs & 2,1410 & 4,6 & 1,0371 & 7,6 \\
\hline Com ECIs & 2,2114 & 1,5 & 1,1159 & 0,6 \\
\hline
\end{tabular}

Tabela 4.1: Deslocamento vertical $\left(m \times 10^{-2}\right)$

linhas. Em seguida são apresentados os resultados obtidos por Moser et al. (2004), o qual emprega ECs e ECIs com funções de forma quadráticas. Por fim, as duas últimas linhas contêm os valores obtidos neste trabalho utilizando a malha da figura 4.16. Primeiramente foram calculados os deslocamentos empregando somente a malha de ECs, igualando os deslocamentos e forças a zero nos nós do perímetro. Em seguida adicionou-se ECIs para modelar a região além da malha original, sendo que toda a sua influência é computada nos nós do perímetro.

Comparando as duas últimas linhas da tabela, conclui-se que a inclusão dos ECIs melhora a precisão dos resultados de forma significativa. Além disto o acréscimo no custo computacional pode ser considerado pequeno, pois os ECIs desenvolvidos neste trabalho não adicionam novos graus de liberdade à malha original. Esta vantagem não ocorre no caso dos ECIs da formulação de Moser et al. (2004), pois seu uso implica em adicionar novos nós à malha. Além disso, conforme pode ser observado na tabela 4.1, a precisão dos elementos quadráticos de Moser et al. (2004) pode ser considerada próxima da obtida com os elementos lineares deste trabalho. Desta forma, conclui-se que elementos lineares 
são suficientes para a simulação deste tipo de problema.

Para melhor comparar a precisão os resultados obtidos com e sem os ECIs, foram feitos vários testes com diversas malhas diferentes. Foi verificado que, caso mais ECs sejam adicionados no perímetro da malha inicial, mesmo sem os ECIs, a resposta se aproxima da exata. Desta forma, foi montada a tabela 4.2 .

\begin{tabular}{|c|c|c|c|c|c|}
\cline { 2 - 6 } \multicolumn{1}{c|}{} & N. nós & Ponto $A$ & Erro (\%) & Ponto $B$ & Erro (\%) \\
\hline Solução exata & & 2,2444 & - & 1,1222 & - \\
\hline \hline${ }^{1}$ Com ECIs & 57 & 2,1869 & 2,6 & 1,0923 & 2,7 \\
\hline${ }^{2}$ Com ECIs & 73 & 2,2114 & 1,5 & 1,1159 & 0,6 \\
\hline \hline Sem ECIs & 57 & 2,0987 & 6,5 & 0,9558 & 14,8 \\
\hline Sem ECIs & 73 & 2,1410 & 4,6 & 1,0371 & 7,6 \\
\hline Sem ECIs & 89 & 2,1684 & 3,4 & 1,0702 & 4,6 \\
\hline Sem ECIs & 105 & 2,1857 & 2,6 & 1,0887 & 3,0 \\
\hline${ }^{1}$ Sem ECIs & 121 & 2,1966 & 2,1 & 1,0998 & 2,0 \\
\hline Sem ECIs & 137 & 2,2076 & 1,8 & 1,1066 & 1,4 \\
\hline Sem ECIs & 153 & 2,2076 & 1,6 & 1,1109 & 1,0 \\
\hline Sem ECIs & 169 & 2,2103 & 1,5 & 1,1135 & 0,8 \\
\hline${ }^{2}$ Sem ECIs & 185 & 2,2119 & 1,4 & 1,1152 & 0,6 \\
\hline
\end{tabular}

Tabela 4.2: Deslocamento vertical para várias malhas $\left(m \times 10^{-2}\right)$

Esta tabela é semelhante à tabela 4.1, mas com uma coluna a mais para indicar o número de nós empregados em cada simulação. As duas linhas seguintes à solução exata são os resultados das modelagens feitas com ECIs, as quais são identificadas com os números 1 e 2, e as demais linhas são os resultados sem ECIs. É possível perceber que os erros diminuem com o aumento do número de nós. Observa-se, por exemplo, que a simulação sem ECIs identificada com o número 1 é a primeira que supera, em precisão, a simulação com ECIs de mesmo número. O mesmo acontece com as linhas identificadas pelo número 2. Considerando o número de nós nestes casos, conclue-se que a utilização dos ECIs permite uma redução de mais de $50 \%$ no número de nós sem prejuízo da precisão dos valores obtidos. Esta constatação é muito relevante para este trabalho, pois seu principal objetivo é a redução do tempo de processamento sem perder a qualidade dos resultados.

\subsubsection{Semi-espaço infinito não homogêneo}

Como neste segundo exemplo é feita uma análise semelhante à do primeiro, apresentase aqui explicações mais resumidas. Na figura 4.15 é ilustrado um semi-espaço infinito 
não homogêneo formado por duas camadas. A espessura da camada superior é de $15 \mathrm{~m}$, com um módulo de elasticidade de $9000 \mathrm{kN} / \mathrm{m}^{2}$ e coeficiente de Poisson 0,5. A camada inferior possui espessura infinita, seu módulo de elasticidade é de $900 \mathrm{kN} / \mathrm{m}^{2}$ e coeficiente de Poisson também de 0,5. Na superfície da camada superior é aplicado um carregamento vertical circular de $2 \mathrm{kN} / \mathrm{m}^{2}$ e $7,5 \mathrm{~m}$ de diâmetro.

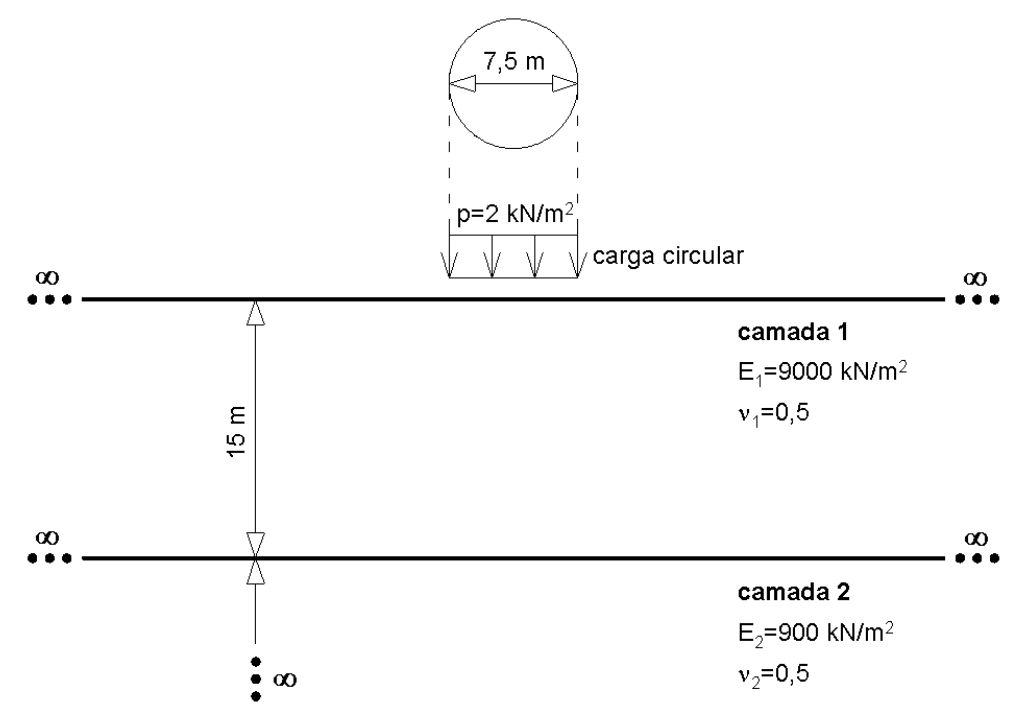

Figura 4.17: Semi-espaço infinito não homogêneo com carregamento circular

Este problema foi simulado empregando uma malha composta por dois trechos iguais, um para a superfície e outro para a interface entre camadas, totalizando 242 nós, 448 ECs e 64 ECIs. O trecho da superfície é ilustrado na figura 4.18, em que o polígono destacado no centro corresponde à área carregada, as linhas tracejadas representam os ECIs, as linhas não tracejadas correspondem aos ECs e os pontos assinalados são os que recebem as contribuições dos ECIs.

No trabalho de Burmister (1945a), no qual este problema é analisado analiticamente, foi obtido para o ponto central da área carregada um deslocamento vertical de 2,5000 $\times$ $10^{-3} \mathrm{~m}$. A simulação com a malha da figura 4.18 , por sua vez, forneceu para o mesmo ponto e direção um valor de $2,5123 \times 10^{-3} \mathrm{~m}$. Isto implica em um erro abaixo de $1 \%$, podendo portanto ser considerado um resultado preciso.

Com o intuito de verificar a redução na malha decorrente da utilização dos ECIs, mais simulações foram feitas para diferentes números de nós e foi montada a tabela 4.3 .

A segunda linha da tabela contém o resultado obtido por Burmister (1945a), escolhido como referência para o cálculo dos erros. A primeira coluna da tabela indica o número de nós empregados em cada simulação, e seu aumento implica em uma maior distância entre 


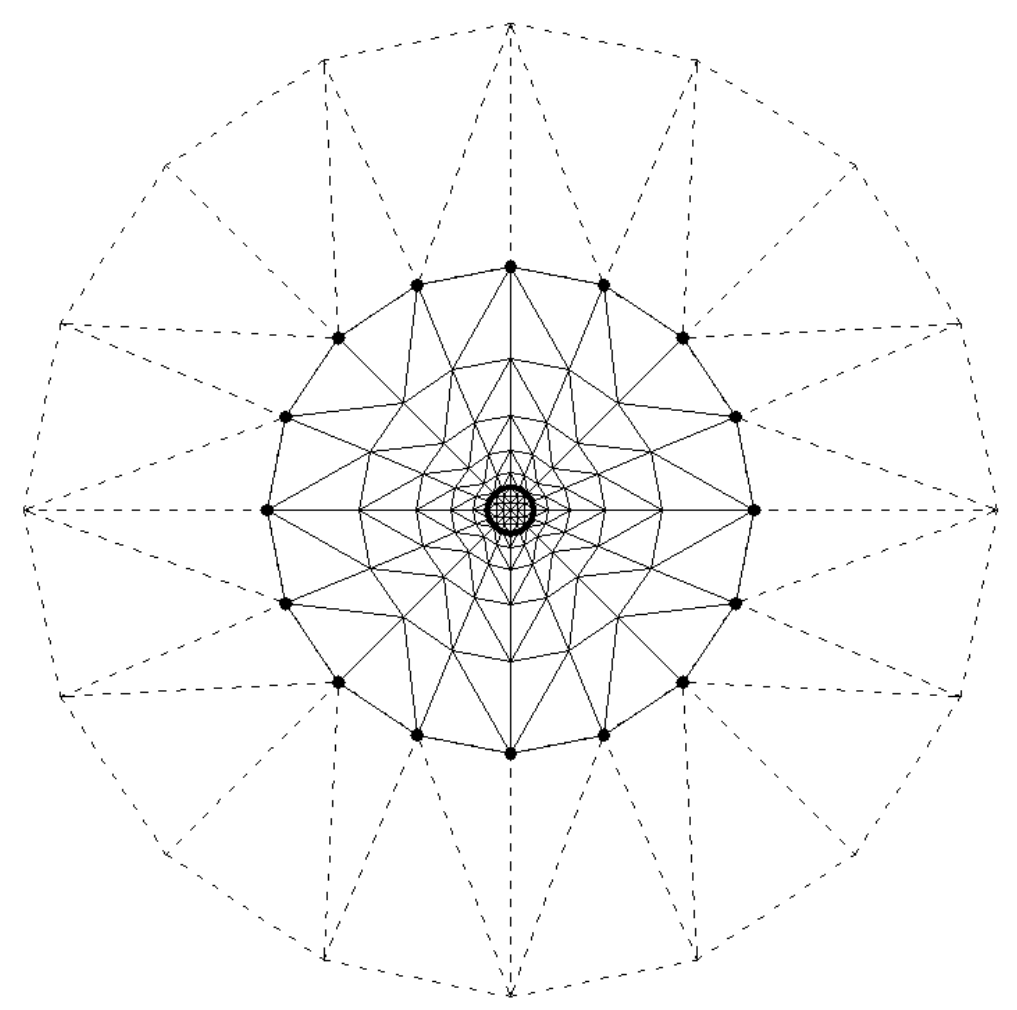

Figura 4.18: Malha da superfície

\begin{tabular}{|c|c|c|c|c|}
\hline N. nós & Sem ECIs & Erro (\%) & Com ECIs & Erro (\%) \\
\hline \hline Burmister & 2,5000 & & 2,5000 & \\
\hline \hline 178 & 1,4965 & 40,1 & 2,1278 & 14,9 \\
\hline 210 & 1,7437 & 30,3 & 2,4110 & 3,6 \\
\hline 242 & 2,0874 & 16,5 & 2,5123 & 0,5 \\
\hline 274 & 2,3865 & 4,5 & 2,5209 & 0,8 \\
\hline 306 & 2,5033 & 0,1 & 2,5212 & 0,8 \\
\hline 338 & 2,5207 & 0,8 & 2,5213 & 0,9 \\
\hline
\end{tabular}

Tabela 4.3: Deslocamento vertical $\left(m \times 10^{-3}\right)$

a área carregada e o perímetro da malha. A segunda coluna contém os valores obtidos empregando uma malha somente com ECs, seguida de seus respectivos erros. Por fim, as duas últimas colunas contém os valores obtidos empregando ECIs e os erros em relação ao valor de referência.

É possível observar que os resultados com e sem ECIs convergem ao valor de referência com o aumento da distância da área carregada ao perímetro. No entanto, quando são utilizados ECIs, o erro cai abaixo de 1 \% com apenas 242 nós, enquanto que são necessários 306 nós para que isto seja conseguido sem ECIs. Desta forma, a utilização dos ECIs permitiu uma redução na malha de aproximadamente $20 \%$. 


\subsection{Considerações finais}

Neste Capítulo foram estudados alguns conceitos básicos relacionados à teoria dos elementos infinitos (EI), com o intuito de aplicar este conhecimento no desenvolvimento de elementos de contorno infinitos (ECI) a partir de elementos de contorno convencionais (EC). Após a introdução, o Capítulo foi iniciado mencionando alguns dos primeiros trabalhos que envolveram EIs. Em seguida, na Seção 4.3, descreveu-se resumidamente a teoria empregada no desenvolvimento de EIs com funções de decaimento. Na sequência, na Seção 4.4, foi abordado um outro tipo de EI, o qual é obtido por meio de funções de mapeamento. A partir desta técnica foi desenvolvida, a partir do EC triangular linear apresentado no Capítulo 2, a formulação de um ECI mapeado.

O Capítulo foi então encerrado com a aplicação da formulação desenvolvida. Nos dois exemplos foi demonstrado que a utilização de ECIs possibilita que a malha seja reduzida sem prejuízo da qualidade dos resultados, justificando que sejam empregados nos demais problemas a serem estudados neste trabalho. 


\section{Capítulo 5}

\section{O método dos elementos finitos}

\subsection{Introdução}

O método dos elementos finitos (MEF) é uma ferramenta numérica utilizada na resolução de diversos problemas de engenharia. Neste trabalho esta ferramenta é empregada na simulação de estruturas compostas por lâminas e barras, em análise estática, elástica e linear. A teoria é apresentada de forma sucinta, referenciando publicações complementares.

A partir do equacionamento básico do problema elástico, obtém-se uma equação diferencial que representa o equilíbrio de um sólido qualquer. Aplicando-se nesta equação técnicas de resíduos ponderados, chega-se a uma expressão que representa os trabalhos interno e externo associados à estrutura em estudo. Divide-se então a estrutura em um número qualquer de subdomínios, denominados elementos finitos. A cada elemento é aplicada a equação dos trabalhos interno e externo, adotando aproximações para os deslocamentos e deformações. O resultado é um sistema de equações para cada elemento, tornando-se possível então montar um único sistema válido para toda a estrutura. Após considerar as condições de contorno do problema é possível resolver este sistema, obtendose deslocamentos em pontos definidos na estrutura em questão.

Este mesmo tema pode ser encontrado em Assan (2003).

\subsection{O Princípio dos Trabalhos Virtuais (PTV)}

O MEF é, de forma geral, a mais poderosa e eficiente ferramenta para análise de problemas de domínio finito e de geometria qualquer. Neste trabalho o MEF é apli- 
cado utilizando-se o método dos deslocamentos, o que implica em aproximar o campo de deslocamentos de cada elemento finito por funções ponderadoras. Estas funções estão relacionadas aos parâmetros nodais do elemento, que são valores de deslocamento nos nós do elemento.

No Capítulo 2 foi descrito sucintamente o problema elástico, e foi obtida a seguinte equação de equilíbrio:

$$
\sigma_{i j, j}+b_{i}=0
$$

Nesta equação, $\sigma_{i j}$ são as componentes de tensão em um ponto qualquer de um sólido tridimensional submetido a cargas externas $b_{i}$. Para equacionar o PTV considera-se a equação 5.1 com condições de contorno essenciais e naturais, conforme ilustrado na figura 5.1 .
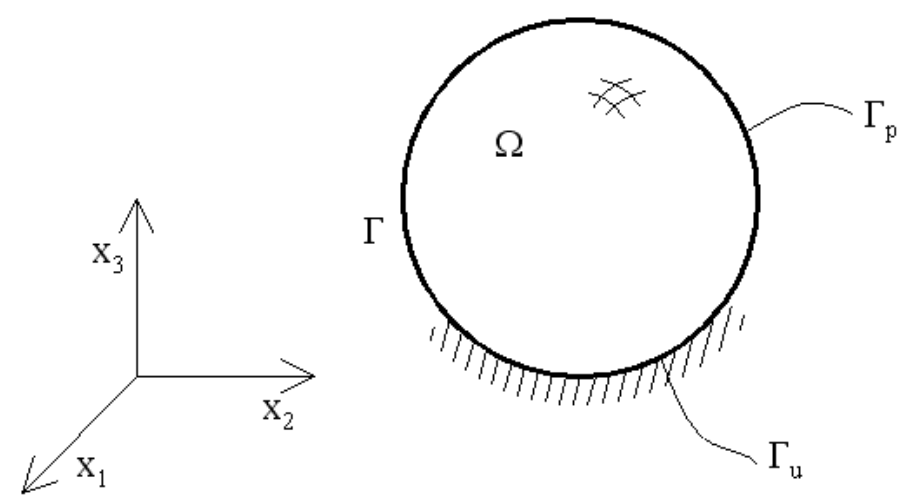

Figura 5.1: Sólido qualquer com condições de contorno arbitrárias

Na figura 5.1, $\Omega$ é o domínio do sólido e $\Gamma$ é seu contorno. As condições de contorno essenciais ou em deslocamento estão aplicadas na parcela $\Gamma_{u}$ do contorno $\Gamma$. Isto é, em $\Gamma_{u}$ :

$$
u_{i}=\bar{u}_{i}
$$

sendo $i$ uma direção do sistema $x_{1} x_{2} x_{3}$.

Por outro lado, as condições de contorno naturais ou em força estão aplicadas no trecho $\Gamma_{p}$ do contorno. Ou seja, em $\Gamma_{p}$ :

$$
p_{i}=\bar{p}_{i}
$$

A soma dos trechos $\Gamma_{u}$ e $\Gamma_{p}$ compõe o contorno total $\Gamma$, ou seja:

$$
\Gamma=\Gamma_{u}+\Gamma_{p}
$$


Definido o problema em questão, a equação 5.1 pode ser trabalhada aplicando-se técnicas de resíduos ponderados. A função ponderadora a ser escolhida corresponde a um campo de deslocamentos virtuais $\tilde{u}_{i}$, o qual deve satisfazer as condições de contorno essenciais impostas ao problema. Definida esta função, escreve-se a expressão:

$$
\int_{\Omega}\left(\sigma_{i j, j}+b_{i}\right) \tilde{u}_{i} d \Omega=0
$$

Integrando a expressão 5.5 por partes, obtém-se:

$$
-\int_{\Omega} \sigma_{i j} \tilde{u}_{i, j} d \Omega+\int_{\Omega} b_{i} \tilde{u}_{i} d \Omega+\int_{\Gamma} p_{i} \tilde{u}_{i} d \Gamma=0
$$

A expressão 5.6, escrita em forma matricial, é equivalente a:

$$
\int_{\Omega}\{\tilde{\varepsilon}\}^{T}\{\sigma\} d \Omega=\int_{\Omega}\{\tilde{u}\}^{T}\{b\} d \Omega+\int_{\Gamma}\{\tilde{u}\}^{T}\{p\} d \Gamma
$$

em que $\{\tilde{\varepsilon}\}$ corresponde ao campo de deformações virtuais decorrentes do campo de deslocamentos virtuais $\{\tilde{u}\}$. A integral à esquerda da equação 5.7 é o trabalho virtual interno mobilizado no sólido, enquanto as integrais à direita são o trabalho virtual externo decorrente das cargas externas aplicadas. O trabalho interno, que está diretamente ligado aos esforços internos, está equacionado em função das tensões $\{\sigma\}$. Para dar continuidade às deduções, é preciso expressar esta integral em função das deformações reais $\{\varepsilon\}$ correspondentes às tensões $\{\sigma\}$. Pode-se relacionar o campo de tensões ao de deformações por meio de relações constitutivas. Estas relações podem ser representadas matricialmente pela relação:

$$
\{\sigma\}=[D]\{\varepsilon\}
$$

A matriz $[D]$ representa um tensor de quarta ordem que traduz as características do material. Substituindo a relação 5.8 na expressão 5.7, obtém-se:

$$
\int_{\Omega}\{\tilde{\varepsilon}\}^{T}[D]\{\varepsilon\} d \Omega=\int_{\Omega}\{\tilde{u}\}^{T}\{b\} d \Omega+\int_{\Gamma}\{\tilde{u}\}^{T}\{p\} d \Gamma
$$

Na expressão 5.9, a função ponderadora $\{\tilde{u}\}$ pode ser qualquer uma que satisfaça as condições de contorno essenciais do problema. Pelo método de Galerkin, descrito em 
Assan (2003), esta função é o próprio campo de deslocamentos real $\{u\}$ do problema. Assim, adotando este procedimento, a expressão 5.9 se torna:

$$
\int_{\Omega}\{\varepsilon\}^{T}[D]\{\varepsilon\} d \Omega=\int_{\Omega}\{u\}^{T}\{b\} d \Omega+\int_{\Gamma}\{u\}^{T}\{p\} d \Gamma
$$

A equação 5.10 representa o Princípio dos Trabalhos Virtuais (PTV). Para resolver esta equação pelo MEF, deve-se dividir o domínio $\Omega$ do sólido em um determinado número de subdomínios $\Omega_{j}$, denominados elementos finitos. Desta forma, cada integral da equação 5.10 passa a ser definida por um somatório das contribuições de todos os elementos finitos, ou seja:

$$
\sum_{j=1}^{n e}\left[\int_{\Omega_{j}}\{\varepsilon\}^{T}[D]\{\varepsilon\} d \Omega\right]=\sum_{j=1}^{n e}\left[\int_{\Omega_{j}}\{u\}^{T}\{b\} d \Omega\right]+\sum_{j=1}^{n e}\left[\int_{\Gamma_{j}}\{u\}^{T}\{p\} d \Gamma\right]
$$

em que ne é o número total de elementos finitos.

As integrais em 5.11 são calculadas empregando funções interpoladoras que aproximam o campo de deslocamentos $\{u\}$ e o campo de deformações $\{\varepsilon\}$ em função dos parâmetros nodais do elemento. Estes parâmetros são os valores de deslocamento nos nós do elemento. Desta forma, para o deslocamento $\{u\}$, escreve-se:

$$
\{u\}=[H(\xi)]\left\{u^{j}\right\}
$$

Na igualdade 5.12, $\xi$ são coordenadas locais definidas em cada elemento finito, $[H(\xi)]$ é uma matriz de funções interpoladoras de deslocamento conhecidas e $\left\{u^{j}\right\}$ é um vetor que contém os valores de deslocamento nos nós do elemento. Da mesma forma, representa-se o campo de deformações $\{\varepsilon\}$ como segue:

$$
\{\varepsilon\}=[B(\xi)]\left\{u^{j}\right\}
$$

em que $[B(\xi)]$ é uma matriz de funções interpoladoras de deformação. Substituindo as 
relações 5.12 e 5.13 em 5.11 , chega-se à expressão:

$$
\begin{gathered}
\sum_{j=1}^{n e}\left[\left\{u^{j}\right\}^{T}\left(\int_{\Omega_{j}}[B]^{T}[D][B] d \Omega\right)\left\{u^{j}\right\}\right]= \\
=\sum_{j=1}^{n e}\left[\left\{u^{j}\right\}^{T} \int_{\Omega_{j}}[H]^{T}\{b\} d \Omega\right]+\sum_{j=1}^{n e}\left[\left\{u^{j}\right\}^{T} \int_{\Gamma_{j}}[H]^{T}\{p\} d \Gamma\right]
\end{gathered}
$$

O passo final é minimizar a energia potencial total expressa na equação 5.14, sendo o lado esquerdo da igualdade a parcela interna da energia e o lado direito a parcela externa. Após este procedimento, a equação se torna:

$$
\sum_{j=1}^{n e}\left[\left[K^{j}\right]\left\{u^{j}\right\}\right]=\sum_{j=1}^{n e}\left[\left\{f^{j}\right\}\right]
$$

em que

$$
\left[K^{j}\right]=\int_{\Omega_{j}}[B]^{T}[D][B] d \Omega
$$

e

$$
\left\{f^{j}\right\}=\int_{\Omega_{j}}[H]^{T}\{b\} d \Omega+\int_{\Gamma_{j}}[H]^{T}\{p\} d \Gamma
$$

Portanto, para cada elemento $j$ é calculada uma matriz $\left[K_{j}\right]$ e um vetor $\left\{f^{j}\right\}$. Como cada vetor $\left\{f^{j}\right\}$ é resultado das cargas externas aplicadas no elemento, ele é denominado como vetor de cargas nodais. O somatório das matrizes $\left[K_{j}\right]$, portanto, relaciona um vetor de deslocamentos a um vetor de cargas nodais, e por este motivo cada matriz $\left[K_{j}\right]$ é denominada como matriz de rigidez do elemento.

Para que as contribuições $\left[K_{j}\right]$ de todos os elementos possam ser somadas é preciso definir um vetor de deslocamentos global, ou seja, que contenha todos os graus de liberdade de todos os elementos finitos. O resultado é uma única matriz de rigidez que guarda a influência de todos os elementos, assim como um único vetor de cargas nodais. Por fim, obtém-se um sistema de equações como segue:

$$
[K]\{u\}=\{f\}
$$

Após aplicar as condições de contorno, este sistema pode ser resolvido e sua solução resulta em valores de deslocamento nos nós de todos os elementos. 


\subsection{Elemento finito utilizado nas estacas}

É apresentado, nesta Seção, o elemento finito que é empregado neste trabalho para simular as estacas. Cada estaca é modelada como um único elemento finito, cujas características gerais são ilustradas na figura 5.2 .

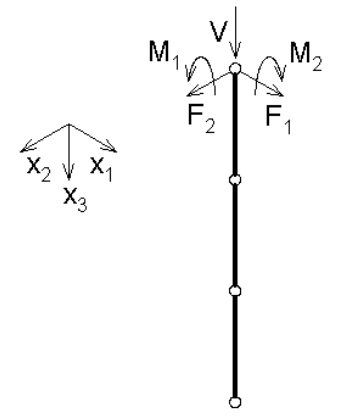

a)

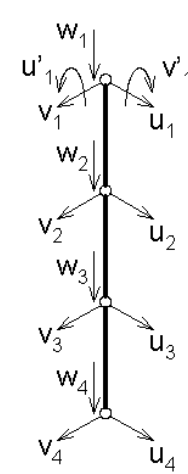

b)

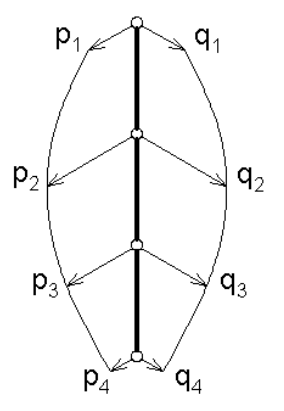

c)

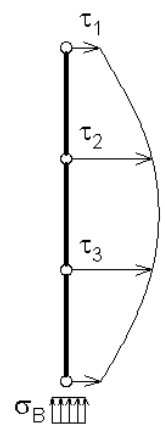

d)

Figura 5.2: Características do elemento finito

A figura, que apresenta um elemento de quatro nós igualmente espaçados ao longo de seu comprimento, é dividida em quatro partes. Em 5.2 a são ilustradas as cargas que podem ser aplicadas no nó do topo da estaca, incluindo forças nas direções $x_{1}, x_{2}$ e $x_{3}$ do sistema de coordenadas globais e momentos em torno de $x_{1}$ e $x_{2}$. Em $5.2 \mathrm{~b}$ estão representados quatorze parâmetros nodais de deslocamento, que incluem deslocamentos nas direções $x_{1}, x_{2}$ e $x_{3}$ nos quatro nós mais rotações em torno de $x_{1}$ e $x_{2}$ no nó do topo. Todas as forças de interação estaca/solo são modeladas como carregamentos distribuídos, assim como ilustrado em 5.2 para as direções horizontais do fuste e em $5.2 \mathrm{~d}$ para as direções verticais do fuste e da base.

Todos os deslocamentos e carregamentos distribuídos são aproximados empregando funções polinomiais, sendo que o grau dos polinômios é escolhido considerando-se o número de parâmetros definidos em cada situação. Para as direções horizontais são definidos cinco parâmetros nodais de deslocamento, portanto o deslocamento $u$ na direção $x_{1}$ e o 
deslocamento $v$ na direção $x_{2}$ podem ser escritos como:

$$
\begin{aligned}
& u=\{\varphi\}^{T}\left\{u_{i}\right\}=\left\{\begin{array}{lllll}
\varphi_{D 1} & \varphi_{\theta 1} & \varphi_{2} & \varphi_{3} & \varphi_{4}
\end{array}\right\}\left\{\begin{array}{c}
u_{1} \\
u_{1}^{\prime} \\
u_{2} \\
u_{3} \\
u_{4}
\end{array}\right\} \\
& v=\{\varphi\}^{T}\left\{v_{i}\right\}=\left\{\begin{array}{lllll}
\varphi_{D 1} & \varphi_{\theta 1} & \varphi_{2} & \varphi_{3} & \varphi_{4}
\end{array}\right\}\left\{\begin{array}{c}
v_{1} \\
v_{1}^{\prime} \\
v_{2} \\
v_{3} \\
v_{4}
\end{array}\right\}
\end{aligned}
$$

Como são cinco parâmetros, o ideal neste caso é empregar polinômios de quarto grau. Utilizando uma variável auxiliar adimensional $\xi$ tal que $\xi=x_{3} / L$, sendo $L$ o comprimento do elemento no sistema global, chega-se às seguintes funções de forma:

$$
\{\varphi\}=\left\{\begin{array}{c}
-\frac{99}{4} \xi^{4}+45 \xi^{3}-\frac{85}{4} \xi^{2}+1 \\
-\frac{9}{2} \xi^{4} L+9 \xi^{3} L-\frac{11}{2} \xi^{2} L+\xi L \\
\frac{81}{2} \xi^{4}-\frac{135}{2} \xi^{3}+27 \xi^{2} \\
-\frac{81}{4} \xi^{4}+27 \xi^{3}-\frac{27}{4} \xi^{2} \\
\frac{9}{2} \xi^{4}-\frac{9}{2} \xi^{3}+\xi^{2}
\end{array}\right\}
$$

O deslocamento $w$ na direção $x_{3}$, por sua vez, deve ser definido a partir de quatro parâmetros. Portanto:

$$
w=\{\phi\}^{T}\left\{w_{i}\right\}=\left\{\begin{array}{llll}
\phi_{1} & \phi_{2} & \phi_{3} & \phi_{4}
\end{array}\right\}\left\{\begin{array}{c}
w_{1} \\
w_{2} \\
w_{3} \\
w_{4}
\end{array}\right\}
$$

Quatro parâmetros implica na utilização de funções de terceiro grau. Empregando 
novamente a variável adimensional $\xi$, obtém-se:

$$
\{\phi\}=\left\{\begin{array}{c}
-\frac{9}{2} \xi^{3}+9 \xi^{2}-\frac{11}{2} \xi+1 \\
\frac{27}{2} \xi^{3}-\frac{45}{2} \xi^{2}+9 \xi \\
-\frac{27}{2} \xi^{3}+18 \xi^{2}-\frac{9}{2} \xi \\
\frac{9}{2} \xi^{3}-\frac{9}{2} \xi^{2}+\xi
\end{array}\right\}
$$

Os carregamentos horizontais do fuste, $q$ na direção $x_{1}$ e $p$ na direção $x_{2}$, são também definidos a partir de quatro parâmetros. Portanto pode-se aproximá-los com as mesmas funções empregadas para os deslocamentos verticais $w$, ou seja:

$$
\begin{aligned}
& q=\{\phi\}^{T}\left\{q_{i}\right\}=\left\{\begin{array}{llll}
\phi_{1} & \phi_{2} & \phi_{3} & \phi_{4}
\end{array}\right\}\left\{\begin{array}{l}
q_{1} \\
q_{2} \\
q_{3} \\
q_{4}
\end{array}\right\} \\
& p=\{\phi\}^{T}\left\{p_{i}\right\}=\left\{\begin{array}{llll}
\phi_{1} & \phi_{2} & \phi_{3} & \phi_{4}
\end{array}\right\}\left\{\begin{array}{c}
p_{1} \\
p_{2} \\
p_{3} \\
p_{4}
\end{array}\right\}
\end{aligned}
$$

O carregamento no fuste $\tau$ na direção $x_{3}$, por sua vez, é definido a partir de três parâmetros. Portanto:

$$
\tau=\{\omega\}^{T}\left\{\tau_{i}\right\}=\left\{\begin{array}{lll}
\omega_{1} & \omega_{2} & \omega_{3}
\end{array}\right\}\left\{\begin{array}{c}
\tau_{1} \\
\tau_{2} \\
\tau_{3}
\end{array}\right\}
$$

Para três parâmetros o ideal é empregar funções de segundo grau. Estas funções são:

$$
\{\omega\}=\left\{\begin{array}{c}
\frac{9}{2} \xi^{2}-\frac{9}{2} \xi+1 \\
-9 \xi^{2}+6 \xi \\
\frac{9}{2} \xi^{2}-\frac{3}{2} \xi
\end{array}\right\}
$$

Por fim, como a carga na base da estaca é definida a partir de um único parâmetro, 
adota-se para ela uma aproximação constante. Isto é:

$$
\sigma=\{1\}\left\{\sigma_{b}\right\}
$$

ou simplesmente:

$$
\sigma=\sigma_{b}
$$

Definidas as funções aproximadoras o próximo passo é escrever a expressão da energia potencial total do elemento, que é dada por:

$$
\Pi=\Upsilon+\Omega^{\prime}
$$

em que $\Upsilon$ representa a energia potencial total de deformação e $\Omega^{\prime}$ representa a energia potencial total das cargas externas. Considerando as características do elemento conforme definidas na figura 5.2, cada parcela da expressão 5.30 pode ser escrita como:

$$
\begin{gathered}
\Upsilon=\frac{E I}{2} L \int_{0}^{1}\left(u^{\prime \prime}\right)^{2} d \xi+\frac{E I}{2} L \int_{0}^{1}\left(v^{\prime \prime}\right)^{2} d \xi+\frac{E A}{2} L \int_{0}^{1}\left(w^{\prime}\right)^{2} d \xi \\
\Omega^{\prime}=L \int_{0}^{1} u q d \xi+L \int_{0}^{1} v p d \xi+L \int_{0}^{1} w \tau d \xi+\int_{A} w_{4} \sigma_{b} d A-F_{1} u_{1}-F_{2} v_{1}-M_{1} u_{1}^{\prime}-M_{2} v_{1}^{\prime}-V w_{1}
\end{gathered}
$$

Nas expressões $5.31 \mathrm{e} 5.32 L$ é o comprimento da estaca, $E$ é seu módulo de elasticidade, $I$ é o momento de inércia da seção transversal e $A$ é sua área. É necessário calcular a derivada da aproximação dos deslocamentos verticais, $w$, e a segunda derivada das aproximações dos deslocamentos horizontais, $u$ e $v$. Estas derivadas são dadas por:

$$
\begin{aligned}
& u^{\prime \prime}=\left\{\varphi^{\prime \prime}\right\}^{T}\left\{u_{i}\right\} \\
& v^{\prime \prime}=\left\{\varphi^{\prime \prime}\right\}^{T}\left\{v_{i}\right\} \\
& w^{\prime}=\left\{\phi^{\prime}\right\}^{T}\left\{w_{i}\right\}
\end{aligned}
$$

O próximo passo é substituir estas derivadas, assim como as demais expressões definidas anteriormente, em cada termo das igualdades 5.31 e 5.32 e desenvolvê-las. Para simplificar a dedução, estuda-se cada contribuição separada das demais para posterior- 
mente analisar como elas devem ser somadas. Inicia-se com a seguinte integral:

$$
\frac{E I}{2} L \int_{0}^{1}\left(u^{\prime \prime}\right)^{2} d \xi=\frac{E I}{2} L \int_{0}^{1}\left\{u_{i}\right\}^{T}\left\{\varphi^{\prime \prime}\right\}^{T}\left\{\varphi^{\prime \prime}\right\}\left\{u_{i}\right\} d \xi=\left\{u_{i}\right\}^{T}\left[K_{h}\right]\left\{u_{i}\right\}
$$

em que

$$
\left[K_{h}\right]=\frac{E I}{2} L \int_{0}^{1}\left\{\varphi^{\prime \prime}\right\}^{T}\left\{\varphi^{\prime \prime}\right\} d \xi
$$

Na sequência, efetua-se uma análise semelhante para todos os outros termos das expressões 5.31 e 5.32 . Desta forma:

$$
\frac{E I}{2} L \int_{0}^{1}\left(v^{\prime \prime}\right)^{2} d \xi=\frac{E I}{2} L \int_{0}^{1}\left\{v_{i}\right\}^{T}\left\{\varphi^{\prime \prime}\right\}^{T}\left\{\varphi^{\prime \prime}\right\}\left\{v_{i}\right\} d \xi=\left\{v_{i}\right\}^{T}\left[K_{h}\right]\left\{v_{i}\right\}
$$

sendo $\left[K_{h}\right]$ dado em 5.37 .

$$
\frac{E A}{2} L \int_{0}^{1}\left(w^{\prime}\right)^{2} d \xi=\frac{E A}{2} L \int_{0}^{1}\left\{w_{i}\right\}^{T}\left\{\phi^{\prime}\right\}^{T}\left\{\phi^{\prime}\right\}\left\{w_{i}\right\} d \xi=\left\{w_{i}\right\}^{T}\left[K_{v}\right]\left\{w_{i}\right\}
$$

em que

$$
\begin{gathered}
{\left[K_{v}\right]=\frac{E A}{2} L \int_{0}^{1}\left\{\phi^{\prime}\right\}^{T}\left\{\phi^{\prime}\right\} d \xi} \\
L \int_{0}^{1} u q d \xi=L \int_{0}^{1}\left\{u_{i}\right\}^{T}\{\varphi\}^{T}\{\phi\}\left\{q_{i}\right\} d \xi=\left\{u_{i}\right\}^{T}\left[J_{h}\right]\left\{q_{i}\right\}
\end{gathered}
$$

com

$$
\begin{gathered}
{\left[J_{h}\right]=L \int_{0}^{1}\{\varphi\}^{T}\{\phi\} d \xi} \\
L \int_{0}^{1} v p d \xi=L \int_{0}^{1}\left\{v_{i}\right\}^{T}\{\varphi\}^{T}\{\phi\}\left\{p_{i}\right\} d \xi=\left\{v_{i}\right\}^{T}\left[J_{h}\right]\left\{p_{i}\right\}
\end{gathered}
$$

sendo $\left[J_{h}\right]$ representado em 5.42 .

$$
L \int_{0}^{1} w \tau d \xi=L \int_{0}^{1}\left\{w_{i}\right\}^{T}\{\phi\}^{T}\{\omega\}\left\{\tau_{i}\right\} d \xi=\left\{w_{i}\right\}^{T}\left[J_{v}\right]\left\{\tau_{i}\right\}
$$

sendo

$$
\left[J_{v}\right]=L \int_{0}^{1}\{\phi\}^{T}\{\omega\} d \xi
$$

E finalmente

$$
\int_{A} \sigma_{b} w_{4} d A=\left[\int_{A} \sigma_{b} d A\right] w_{4}=R w_{4}
$$

em que $R$ é a força resultante axial na ponta da estaca. A expressão da energia potencial 
total se torna então:

$$
\begin{gathered}
\Pi=\left\{u_{i}\right\}^{T}\left[K_{h}\right]\left\{u_{i}\right\}+\left\{v_{i}\right\}^{T}\left[K_{h}\right]\left\{v_{i}\right\}+\left\{w_{i}\right\}^{T}\left[K_{v}\right]\left\{w_{i}\right\}+\left\{u_{i}\right\}^{T}\left[J_{h}\right]\left\{q_{i}\right\}+ \\
+\left\{v_{i}\right\}^{T}\left[J_{h}\right]\left\{p_{i}\right\}+\left\{w_{i}\right\}^{T}\left[J_{v}\right]\left\{\tau_{i}\right\}+R w_{4}-F_{1} u_{1}-F_{2} v_{1}-M_{1} u_{1}^{\prime}-M_{2} v_{1}^{\prime}-V w_{1}
\end{gathered}
$$

O passo seguinte é minimizar a expressão da energia potencial total em função dos parâmetros nodais de deslocamento. Isto é feito calculando-se a derivada em relação a cada um dos quatorze graus de liberdade e igualando a zero, resultando em um sistema com quatorze equações. Desta forma são obtidas todas as contribuições da matriz de rigidez e do vetor de cargas nodais do elemento finito. As contribuições na matriz de rigidez nas direções $x_{1}$ e $x_{2}$ são:

$$
\left[K^{1}\right]=\frac{E I}{40 L^{3}}\left[\begin{array}{ccccc}
23722 & 4084 L & -42876 & 26838 & -7684 \\
4084 L & 808 L^{2} & -6912 L & 3996 L & -1168 L \\
-42876 & -6912 L & 81648 & -55404 & 16632 \\
26838 & 3996 L & -55404 & 42282 & -13716 \\
-7684 & -1168 L & 16632 & -13716 & 4768
\end{array}\right]
$$

e as contribuições dos carregamentos distribuídos nestas mesmas direções no vetor de cargas nodais são:

$$
\begin{gathered}
\left\{f^{q}\right\}=\left[Q^{1}\right]\left\{q_{i}\right\}=\frac{L}{6720}\left[\begin{array}{cccc}
721 & 495 & -45 & 285 \\
38 L & 18 L & 18 L & 38 L \\
54 & 2430 & -486 & -486 \\
27 & -243 & 2673 & 567 \\
38 & -162 & 378 & 474
\end{array}\right]\left\{\begin{array}{l}
q_{1} \\
q_{2} \\
q_{3} \\
q_{4}
\end{array}\right\} \\
\left\{f^{p}\right\}=\left[Q^{1}\right]\left\{p_{i}\right\}=\frac{L}{6720}\left[\begin{array}{cccc}
721 & 495 & -45 & 285 \\
38 L & 18 L & 18 L & 38 L \\
54 & 2430 & -486 & -486 \\
27 & -243 & 2673 & 567 \\
38 & -162 & 378 & 474
\end{array}\right]\left\{\begin{array}{l}
p_{1} \\
p_{2} \\
p_{3} \\
p_{4}
\end{array}\right\}
\end{gathered}
$$


A contribuição na matriz de rigidez na direção $x_{3}$ é:

$$
\left[K^{2}\right]=\frac{E A}{40 L}\left[\begin{array}{cccc}
148 & -189 & 54 & -13 \\
-189 & 432 & -297 & 54 \\
54 & -297 & 432 & -189 \\
-13 & 54 & -189 & 148
\end{array}\right]
$$

e a contribuição no vetor de cargas nodais devido aos carregamentos distribuídos nesta direção é:

$$
\left\{f^{\tau}\right\}=\left[Q^{2}\right]\left\{\tau_{i}\right\}=\frac{L}{80}\left[\begin{array}{cccc}
7 & 2 & 1 & 0 \\
3 & 36 & -9 & 0 \\
3 & -18 & 45 & 0 \\
7 & -20 & 23 & \frac{80}{L}
\end{array}\right]\left\{\begin{array}{c}
\tau_{1} \\
\tau_{2} \\
\tau_{3} \\
R
\end{array}\right\}
$$

As demais contribuições do vetor de cargas nodais podem ser diretamente somadas nas posições correspondentes do vetor do sistema de equações final. Para que este sistema possa ser montado, deve-se primeiramente escolher a ordem dos quatorze graus de liberdade do elemento. Define-se então o vetor global de deslocamentos como segue:

$$
\{u\}^{T}=\left\{\begin{array}{llllllllllllll}
u_{1} & v_{1} & w_{1} & u_{1}^{\prime} & v_{1}^{\prime} & u_{2} & v_{2} & w_{2} & u_{3} & v_{3} & w_{3} & u_{4} & v_{4} & w_{4}
\end{array}\right\}
$$

Na sequência, cria-se um vetor no qual são posicionadas as cargas aplicadas no topo da estaca conforme a ordem definida para os graus de liberdade. Este vetor é:

$$
\{f\}^{T}=\left\{\begin{array}{llllllllllllll}
F_{1} & F_{2} & V & M_{1} & M_{2} & 0 & 0 & 0 & 0 & 0 & 0 & 0 & 0 & 0
\end{array}\right\}
$$

O passo seguinte é montar a matriz de rigidez global do elemento, com quatorze linhas e colunas. Todos os termos diferentes de zero estão presentes nas matrizes $\left[K^{1}\right]$ e $\left[K^{2}\right]$, apresentadas nas igualdades 5.48 e 5.51 , respectivamente. Portanto, a matriz $[K]$ global 
é organizada abaixo referenciando as matrizes menores que já foram escritas:

$$
\left[\begin{array}{cccccccccccccc}
K_{11}^{1} & 0 & 0 & K_{12}^{1} & 0 & K_{13}^{1} & 0 & 0 & K_{14}^{1} & 0 & 0 & K_{15}^{1} & 0 & 0 \\
0 & K_{11}^{1} & 0 & 0 & -K_{12}^{1} & 0 & K_{13}^{1} & 0 & 0 & K_{14}^{1} & 0 & 0 & K_{15}^{1} & 0 \\
0 & 0 & K_{11}^{2} & 0 & 0 & 0 & 0 & K_{12}^{2} & 0 & 0 & K_{13}^{2} & 0 & 0 & K_{14}^{2} \\
K_{21}^{1} & 0 & 0 & K_{22}^{1} & 0 & K_{23}^{1} & 0 & 0 & K_{24}^{1} & 0 & 0 & K_{25}^{1} & 0 & 0 \\
0 & -K_{21}^{1} & 0 & 0 & K_{22}^{1} & 0 & -K_{23}^{1} & 0 & 0 & -K_{24}^{1} & 0 & 0 & -K_{25}^{1} & 0 \\
K_{31}^{1} & 0 & 0 & K_{32}^{1} & 0 & K_{33}^{1} & 0 & 0 & K_{34}^{1} & 0 & 0 & K_{35}^{1} & 0 & 0 \\
0 & K_{31}^{1} & 0 & 0 & -K_{32}^{1} & 0 & K_{33}^{1} & 0 & 0 & K_{34}^{1} & 0 & 0 & K_{35}^{1} & 0 \\
0 & 0 & K_{21}^{2} & 0 & 0 & 0 & 0 & K_{22}^{2} & 0 & 0 & K_{23}^{2} & 0 & 0 & K_{24}^{2} \\
K_{41}^{1} & 0 & 0 & K_{42}^{1} & 0 & K_{43}^{1} & 0 & 0 & K_{44}^{1} & 0 & 0 & K_{45}^{1} & 0 & 0 \\
0 & K_{41}^{1} & 0 & 0 & -K_{42}^{1} & 0 & K_{43}^{1} & 0 & 0 & K_{44}^{1} & 0 & 0 & K_{45}^{1} & 0 \\
0 & 0 & K_{31}^{2} & 0 & 0 & 0 & 0 & K_{32}^{2} & 0 & 0 & K_{33}^{2} & 0 & 0 & K_{34}^{2} \\
K_{51}^{1} & 0 & 0 & K_{52}^{1} & 0 & K_{53}^{1} & 0 & 0 & K_{54}^{1} & 0 & 0 & K_{55}^{1} & 0 & 0 \\
0 & K_{51}^{1} & 0 & 0 & -K_{52}^{1} & 0 & K_{53}^{1} & 0 & 0 & K_{54}^{1} & 0 & 0 & K_{55}^{1} & 0 \\
0 & 0 & K_{41}^{2} & 0 & 0 & 0 & 0 & K_{42}^{2} & 0 & 0 & K_{43}^{2} & 0 & 0 & K_{44}^{2}
\end{array}\right]
$$

É importante observar que alguns dos termos referentes à rotação em torno do eixo $x_{2}$ têm o sinal trocado. Isso é necessário por causa dos sentidos adotados como positivos, sendo a rotação em torno do eixo $x_{1}$ positiva e a rotação em torno do eixo $x_{2}$ negativa.

Por fim, deve-se agrupar em um único vetor todas as contribuições dos carregamentos distribuídos. Para facilitar este procedimento, monta-se uma matriz global $[Q]$ a partir das matrizes $\left[Q_{1}\right]$ e $\left[Q_{2}\right]$ utilizadas nas expressões 5.49, 5.50 e 5.52, O resultado é uma 
matriz de quatorze linhas e doze colunas, como segue:

$$
\left[\begin{array}{cccccccccccc}
Q_{11}^{1} & 0 & 0 & Q_{12}^{1} & 0 & 0 & Q_{13}^{1} & 0 & 0 & Q_{14}^{1} & 0 & 0 \\
0 & Q_{11}^{1} & 0 & 0 & Q_{12}^{1} & 0 & 0 & Q_{13}^{1} & 0 & 0 & Q_{14}^{1} & 0 \\
0 & 0 & Q_{11}^{2} & 0 & 0 & Q_{12}^{2} & 0 & 0 & Q_{13}^{2} & 0 & 0 & Q_{14}^{2} \\
Q_{21}^{1} & 0 & 0 & Q_{22}^{1} & 0 & 0 & Q_{23}^{1} & 0 & 0 & Q_{24}^{1} & 0 & 0 \\
0 & -Q_{21}^{1} & 0 & 0 & -Q_{22}^{1} & 0 & 0 & -Q_{23}^{1} & 0 & 0 & -Q_{24}^{1} & 0 \\
Q_{31}^{1} & 0 & 0 & Q_{32}^{1} & 0 & 0 & Q_{33}^{1} & 0 & 0 & Q_{34}^{1} & 0 & 0 \\
0 & Q_{31}^{1} & 0 & 0 & Q_{32}^{1} & 0 & 0 & Q_{33}^{1} & 0 & 0 & Q_{34}^{1} & 0 \\
0 & 0 & Q_{21}^{2} & 0 & 0 & Q_{22}^{2} & 0 & 0 & Q_{23}^{2} & 0 & 0 & Q_{24}^{2} \\
Q_{41}^{1} & 0 & 0 & Q_{42}^{1} & 0 & 0 & Q_{43}^{1} & 0 & 0 & Q_{44}^{1} & 0 & 0 \\
0 & Q_{41}^{1} & 0 & 0 & Q_{42}^{1} & 0 & 0 & Q_{43}^{1} & 0 & 0 & Q_{44}^{1} & 0 \\
0 & 0 & Q_{31}^{2} & 0 & 0 & Q_{32}^{2} & 0 & 0 & Q_{33}^{2} & 0 & 0 & Q_{34}^{2} \\
Q_{51}^{1} & 0 & 0 & Q_{52}^{1} & 0 & 0 & Q_{53}^{1} & 0 & 0 & Q_{54}^{1} & 0 & 0 \\
0 & Q_{51}^{1} & 0 & 0 & Q_{52}^{1} & 0 & 0 & Q_{53}^{1} & 0 & 0 & Q_{54}^{1} & 0 \\
0 & 0 & Q_{41}^{2} & 0 & 0 & Q_{42}^{2} & 0 & 0 & Q_{43}^{2} & 0 & 0 & Q_{44}^{2}
\end{array}\right]
$$

Novamente, como a rotação em torno do eixo $x_{1}$ positiva e a rotação em torno do eixo $x_{2}$ negativa, alguns termos aparecem com o sinal trocado. Montada a matriz global $[Q]$, ordena-se os valores nodais dos carregamentos distribuídos como segue:

$$
\{s\}^{T}=\left\{\begin{array}{llllllllllll}
q_{1} & p_{1} & \tau_{1} & q_{2} & p_{2} & \tau_{2} & q_{3} & p_{3} & \tau_{3} & q_{4} & p_{4} & R
\end{array}\right\}
$$

O sistema de equações global pode então ser escrito utilizando os vetores 5.53, 5.54 e 5.57 e as matrizes 5.55 e 5.56. O resultado é:

$$
[K]\{u\}=\{f\}-[Q]\{s\}
$$

$\mathrm{Ou}$

$$
[K]\{u\}=\{f\}-\{r\}
$$

em que $\{r\}$ representa os carregamentos distribuídos em termos de cargas nodais concentradas.

A equação 5.58 representa uma única estaca formulada com o MEF, a qual deverá ser 
posteriormente acoplada formulação do método dos elementos de contorno (MEC). Caso existam duas ou mais estacas, suas equações são agrupadas em um único bloco para que sejam acopladas de forma mais simplificada. Caso sejam duas estacas, por exemplo, o agrupamento é feito da seguinte forma:

$$
\left[\begin{array}{cc}
K_{1} & 0 \\
0 & K_{2}
\end{array}\right]\left\{\begin{array}{l}
u_{1} \\
u_{2}
\end{array}\right\}=\left\{\begin{array}{l}
f_{1} \\
f_{2}
\end{array}\right\}-\left[\begin{array}{cc}
Q_{1} & 0 \\
0 & Q_{2}
\end{array}\right]\left\{\begin{array}{l}
s_{1} \\
s_{2}
\end{array}\right\}
$$

sendo que os termos com índice subscrito 1 se referem a uma das estacas e os com índice 2 se referem à outra. Esta equação pode ser extendida para um número qualquer de estacas como segue:

$$
\left[\begin{array}{cccc}
K_{1} & 0 & \ldots & 0 \\
0 & K_{2} & \cdots & 0 \\
\vdots & \vdots & \ddots & \vdots \\
0 & 0 & \cdots & K_{n}
\end{array}\right]\left\{\begin{array}{c}
u_{1} \\
u_{2} \\
\vdots \\
u_{n}
\end{array}\right\}=\left\{\begin{array}{c}
f_{1} \\
f_{2} \\
\vdots \\
f_{n}
\end{array}\right\}-\left[\begin{array}{cccc}
Q_{1} & 0 & \cdots & 0 \\
0 & Q_{2} & \cdots & 0 \\
\vdots & \vdots & \ddots & \vdots \\
0 & 0 & \cdots & Q_{n}
\end{array}\right]\left\{\begin{array}{c}
s_{1} \\
s_{2} \\
\vdots \\
s_{n}
\end{array}\right\}
$$

em que $n$ é o número total de estacas.

\subsection{Elementos finitos laminares}

Nesta Seção é feita uma breve descrição da formulação empregada nos elementos finitos laminares que são utilizados na superestrutura do programa utilizado neste trabalho. A combinação destes elementos permite a simulação de uma grande variedade de estruturas, como por exemplo silos e galpões. Esta abrangência se dá pela da combinação do elemento de placa DKT com um elemento de membrana, como será visto mais adiante.

Podem ser consideradas laminares estruturas tridimensionais nas quais uma dimensão é muito menor que as outras duas. São exemplos disto placas, paredes e estruturas em geral formadas por estes dois subsistemas.

Na formulação utilizada neste trabalho, são consideradas as hipóteses de Kirchoff-Love. Estas são:

- A espessura da lâmina é pequena quando comparada às suas demais dimensões e aos raios de curvatura de sua superfície média. 
- As tensões normais à superfície média são desprezíveis em relação às demais.

- Um ponto pertencente a uma reta ortogonal ao plano médio indeformado continua, após a lâmina ter se deformado, pertencendo à mesma reta ortogonal ao plano médio deformado.

- Os deslocamentos normais ao plano médio são pequenos quando comparados à espessura da lâmina.

Partindo destas hipóteses, o campo de deslocamentos em um elemento finito laminar pode ser escrito como:

$$
\{u\}=\left\{\begin{array}{c}
u\left(x_{1}, x_{2}, x_{3}\right) \\
v\left(x_{1}, x_{2}, x_{3}\right) \\
w\left(x_{1}, x_{2}, x_{3}\right)
\end{array}\right\}=\left\{\begin{array}{c}
u_{0}\left(x_{1}, x_{2}\right)-x_{3} \frac{\partial w_{0}}{\partial x_{1}} \\
v_{0}\left(x_{1}, x_{2}\right)-x_{3} \frac{\partial w_{0}}{\partial x_{2}} \\
w_{0}\left(x_{1}, x_{2}\right)
\end{array}\right\}
$$

Na igualdade 5.62, os deslocamentos $u$ e $u_{0}$ encontram-se na direção do eixo $x_{1}$, os deslocamentos $v$ e $v_{0}$ estão na direção do eixo $x_{2}$ e os deslocamentos $w$ e $w_{0}$ estão na direção do eixo $x_{3}$. Os deslocamentos $u, v$ e $w$ se referem a um ponto $P$ qualquer no domínio da placa, e os deslocamentos $u_{0}, v_{0}$ e $w_{0}$ ocorrem na projeção $P^{\prime}$ do ponto $P$ no plano médio da placa deformada. Estas informações podem ser visualizadas na figura 5.3 .
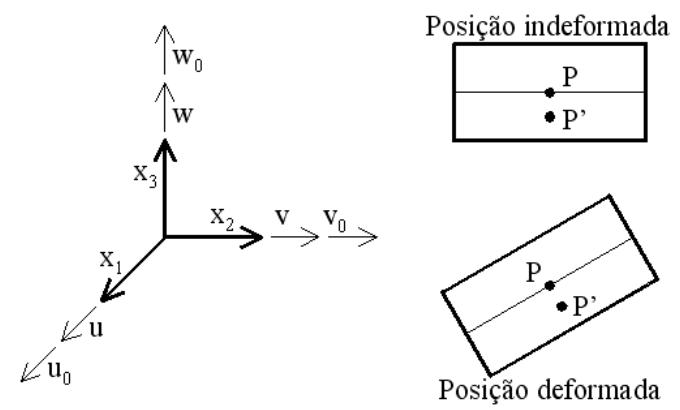

Figura 5.3: Direção dos deslocamentos e posição dos pontos $P$ e $P^{\prime}$

Ao determinar o campo de deformações, deve-se separar as parcelas referentes ao efeito da flexão e ao efeito de membrana. As deformações são dadas então por:

$$
\{\varepsilon\}=\left\{\varepsilon_{m}\right\}+\left\{\varepsilon_{f}\right\}=\left\{\begin{array}{c}
\frac{\partial u_{0}}{\partial x_{1}} \\
\frac{\partial v_{0}}{\partial x_{2}} \\
\frac{\partial u_{0}}{\partial x_{2}}+\frac{\partial v_{0}}{\partial x_{1}}
\end{array}\right\}-x_{3}\left\{\begin{array}{c}
\frac{\partial^{2} w_{0}}{\partial x_{1}^{2}} \\
\frac{\partial^{2} w_{0}}{\partial x_{2}^{2}} \\
2 \frac{\partial^{2} w_{0}}{\partial x_{1} \partial x_{2}}
\end{array}\right\}
$$


Na igualdade 5.63, o índice subscrito $m$ indica efeito de membrana e o índice subscrito $f$ indica efeito de flexão.

Para determinar a matriz de rigidez do elemento considerado, deve-se determinar as matrizes $[B]$ e $[D]$ que aparecem na equação 5.16. A obtenção de $[D]$ parte da aplicação das relações constitutivas entre tensão e deformação. Ou seja:

$$
\{\sigma\}=[D]\{\varepsilon\}
$$

A obtenção da matriz $[B]$ envolve as aproximações adotadas para os deslocamentos no elemento. Estas são dependentes dos parâmetros nodais do elemento, e podem ser escritas como:

$$
\{u\}=[\varphi]\left\{u^{j}\right\}=\left[\begin{array}{cc}
\varphi_{m} & 0 \\
0 & \varphi_{f}
\end{array}\right]\left\{\begin{array}{l}
u_{m}^{j} \\
u_{f}^{j}
\end{array}\right\}
$$

O subvetor $u_{m}^{j}$ contém os graus de liberdade do elemento finito $j$ referentes ao efeito de membrana, e o subvetor $u_{f}^{j}$ contém os graus de liberdade de $j$ referentes ao efeito de flexão. As submatrizes $\varphi_{f}$ e $\varphi_{m}$ contêm as funções de forma adotadas para a parcela de flexão e de membrana, respectivamente. A aproximação para as deformações é então obtida a partir da relação 5.63 , chegando a:

$$
\{\varepsilon\}=[B]\left\{u^{j}\right\}=\left[\begin{array}{ll}
B_{m} & x_{3} B_{f}
\end{array}\right]\left\{\begin{array}{c}
u_{m}^{j} \\
u_{f}^{j}
\end{array}\right\}
$$

O termo $\left[B_{m}\right]$ é a matriz que contém as funções de interpolação referentes ao efeito de membrana e o termo $\left[B_{f}\right]$ é a matriz que contém as funções de interpolação referentes ao efeito de flexão. Substituindo as matrizes $[B]$ e $[D]$ na expressão 5.16 , pode-se obter a matriz de rigidez do elemento laminar pela expressão:

$$
\left[K^{j}\right]=\int_{\Omega_{j}}[B]^{T}[D][B] d \Omega
$$

em que $\Omega_{j}$ é o domínio do elemento. Após efetuar a integral, obtém-se:

$$
\left[K^{j}\right]=\left[\begin{array}{cc}
K_{m} & 0 \\
0 & K_{f}
\end{array}\right]
$$


As submatrizes de zeros identificam uma importante característica desta formulação, que é a independência entre os efeitos de membrana e flexão.

\subsection{Graus de liberdade do elemento finito laminar}

Os elementos finitos laminares utilizados neste trabalho são triangulares e com três nós, havendo um nó em cada vértice. Dos graus de liberdade associados a cada nó, três são referentes ao elemento finito de membrana, FF, e três são referentes ao elemento finito de flexão, DKT. O elemento finito de membrana com os graus de liberdade de seus nós pode ser visualizado na figura 5.4 .
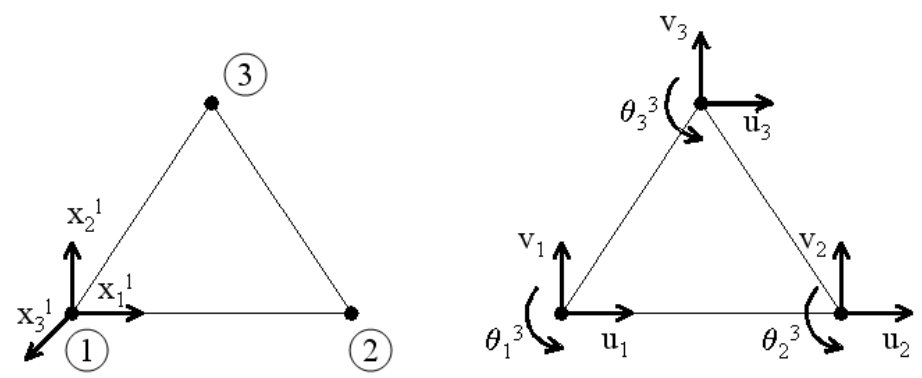

Figura 5.4: Graus de liberdade do elemento finito de membrana FF

Na figura 5.4 está ilustrado o sistema de coordenadas local $x_{i}^{l}$, a numeração dos nós e os graus de liberdade de cada nó referentes ao elemento finito de membrana. No sistema local, a origem está localizada no nó número 1 do elemento. O eixo $x_{1}^{l}$ é alinhado com o lado do elemento cujas extremidades são os nós 1 e 2 , orientado para o nó 2 . O eixo $x_{2}^{l}$ é perpendicular ao eixo $x_{1}^{l}$, e pertence ao plano do elemento. Por fim, o eixo $x_{3}^{l}$ é ortogonal ao plano do elemento.

Em cada nó do elemento de membrana existem três graus de liberdade. O deslocamento $u$ ocorre na direção do eixo local $x_{1}^{l}$ e o deslocamento $v$ ocorre na direção do eixo local $x_{2}^{l}$. A rotação $\theta$ se dá em torno do eixo $x_{3}^{l}$, sendo positiva do eixo $x_{1}^{l}$ para o eixo $x_{2}^{l}$. Os graus de liberdade nos nós do elemento organizados em forma de vetor são:

$$
\left\{u_{m}\right\}^{T}=\left\{\begin{array}{lllllllll}
u_{1} & v_{1} & \theta_{1}^{3} & u_{2} & v_{2} & \theta_{2}^{3} & u_{3} & v_{3} & \theta_{3}^{3}
\end{array}\right\}
$$

O elemento finito utilizado neste trabalho para computar o efeito da flexão foi o DKT, conforme mencionado anteriormente. Este elemento com seus graus de liberdade está ilustrado na figura 5.5 

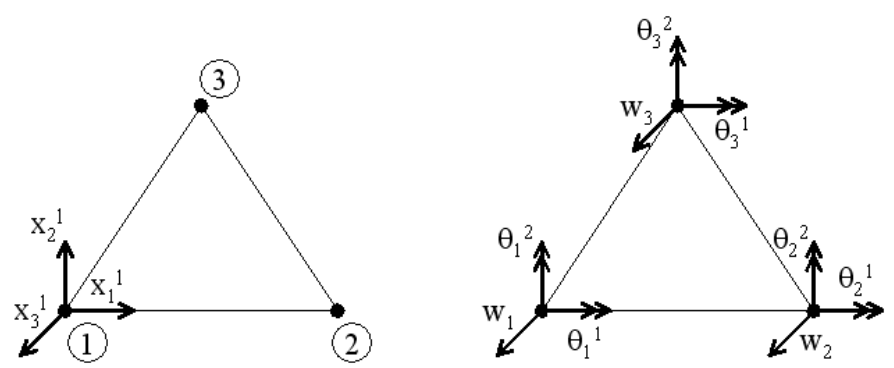

Figura 5.5: Graus de liberdade do elemento finito DKT

Nas rotações $\theta$, indicadas nos nós do elemento DKT na figura 5.5, o índice subscrito indica o número do nó e o índice sobrescrito indica o número do eixo local em torno do qual se dá a rotação. Os deslocamentos $w$ são na direção do eixo local $x_{3}^{l}$. Estes graus de liberdade podem ser listados em um vetor como segue:

$$
\left\{u_{f}\right\}^{T}=\left\{\begin{array}{lllllllll}
w_{1} & \theta_{1}^{1} & \theta_{1}^{2} & w_{2} & \theta_{1}^{1} & \theta_{2}^{2} & w_{3} & \theta_{1}^{1} & \theta_{3}^{2}
\end{array}\right\}
$$

O elemento finito de membrana indicado na figura 5.4 em conjunto com o elemento finito DKT indicado na figura 5.5 compõem o elemento finito laminar DKT/FF, que é utilizado neste trabalho. Este elemento com todos os seus graus de liberdade está ilustrado na figura 5.6 .
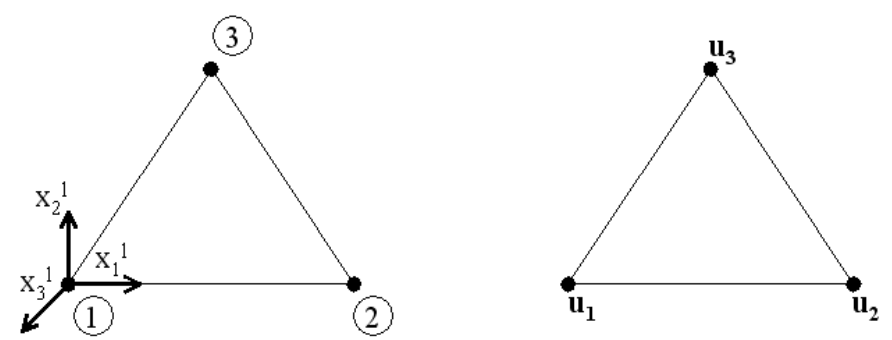

Figura 5.6: Graus de liberdade do elemento finito laminar DKT/FF

Os deslocamentos $\mathbf{u}$ indicados na figura 5.6 são vetores que contêm os graus de liberdade de cada nó. Estes vetores são escritos a seguir:

$$
\begin{aligned}
& \left\{u_{1}\right\}^{T}=\left\{\begin{array}{llllll}
u_{1} & v_{1} & \theta_{1}^{3} & w_{1} & \theta_{1}^{1} & \theta_{1}^{2}
\end{array}\right\} \\
& \left\{u_{2}\right\}^{T}=\left\{\begin{array}{llllll}
u_{2} & v_{2} & \theta_{2}^{3} & w_{2} & \theta_{2}^{1} & \theta_{2}^{2}
\end{array}\right\} \\
& \left\{u_{3}\right\}^{T}=\left\{\begin{array}{llllll}
u_{3} & v_{3} & \theta_{3}^{3} & w_{3} & \theta_{3}^{1} & \theta_{3}^{2}
\end{array}\right\}
\end{aligned}
$$


São seis graus de liberdade por nó, totalizando os dezoito graus de liberdade do elemento finito $\mathrm{DKT} / \mathrm{FF}$.

\subsection{Rotação de eixos}

A matriz de rigidez do elemento finito DKT/FF pode ser obtida pela expressão 5.68 . a partir das submatrizes $\left[K_{m}\right]$ e $\left[K_{f}\right]$. A teoria envolvida na obtenção destas matrizes é extensa e expô-la neste texto desviaria muito dos objetivos deste trabalho. O desenvolvimento para a obtenção da matriz $\left[K_{m}\right]$ pode ser encontrado em Bergan e Felippa (1985), onde é utilizada a formulação livre. As deduções para a obtenção da matriz $\left[K_{f}\right]$, referentes ao elemento DKT, podem ser encontradas em Batoz (1980).

Após deduzir a matriz de rigidez do elemento DKT/FF, é preciso definir como esta matriz pode ser rotacionada do sistema de coordenadas local para o global. Isto é necessário porque a matriz definida para cada elemento finito da estrutura deve ser rotacionada antes de computar sua influência na matriz de rigidez global da estrutura. Para formular este problema, considera-se um elemento finito genérico orientado segundo uma direção qualquer, conforme mostrado na figura 5.7 .

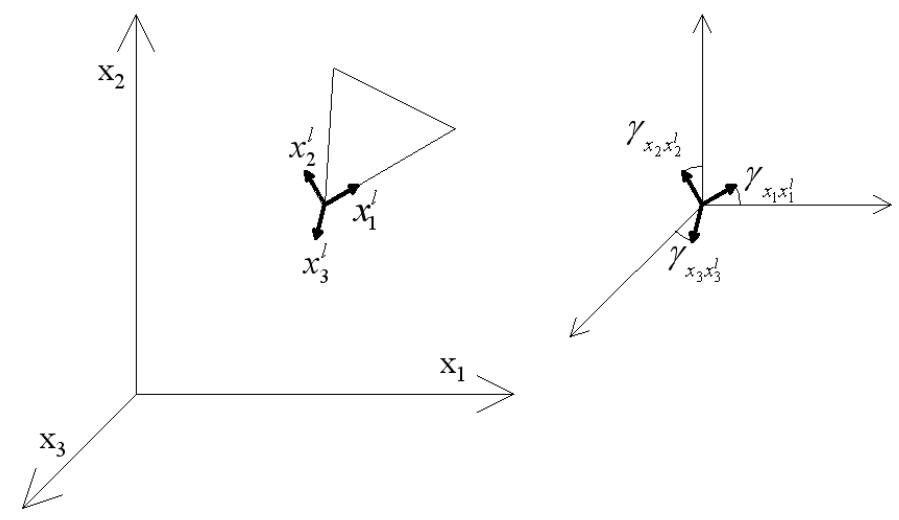

Figura 5.7: Sistema de coordenadas global $x_{1} x_{2} x_{3}$ e local $x_{1}^{l} x_{2}^{l} x_{3}^{l}$

O sistema de coordenadas local $x_{1}^{l} x_{2}^{l} x_{3}^{l}$ pode ser relacionado ao sistema de coordenadas global $x_{1} x_{2} x_{3}$ por meio de uma matriz de rotação $\bar{\beta}$. Assim, escreve-se a igualdade:

$$
\left\{x_{i}^{l}\right\}=[\bar{\beta}]\left\{x_{i}\right\}
$$


$\mathrm{Ou}$

$$
\left\{\begin{array}{l}
x_{1}^{l} \\
x_{2}^{l} \\
x_{3}^{l}
\end{array}\right\}=[\bar{\beta}]\left\{\begin{array}{l}
x_{1} \\
x_{2} \\
x_{3}
\end{array}\right\}
$$

A matriz de rotação $\bar{\beta}$ é dada por:

$$
[\bar{\beta}]=\left[\begin{array}{lll}
\gamma_{11} & \gamma_{12} & \gamma_{13} \\
\gamma_{21} & \gamma_{22} & \gamma_{23} \\
\gamma_{31} & \gamma_{32} & \gamma_{33}
\end{array}\right]
$$

em que os temos $\gamma_{i j}$ correspondem aos co-senos diretores entre os eixos locais e globais. Após determinar a matriz de rotação $\bar{\beta}$, é possível determinar a matriz a ser utlizada para rotacionar a matriz de rigidez do elemento DKT/FF. Esta matriz é:

$$
[\beta]=\left[\begin{array}{ll}
\bar{\beta} & 0 \\
0 & \bar{\beta}
\end{array}\right]
$$

A partir da matriz 5.77, pode-se rotacionar a matriz do elemento aplicando a expressão:

$$
\left[\bar{K}^{j}\right]=[\beta]^{T}\left[K^{j}\right][\beta]
$$

A matriz $\left[\bar{K}^{j}\right]$, calculada pela expressão 5.78 para cada elemento, está pronta para ser computada na matriz de rigidez global da estrutura.

\subsection{Elementos utilizados no edifício}

A formulação a ser empregada no edifício é a mesma do trabalho de Ribeiro (2005). As vigas e pilares são representadas por elementos unidimensionais com seis graus de liberdade por nó. Estes graus de liberdade são os mesmos do elemento laminar descrito na Seção 5.5, o qual é empregado para representar as estruturas planas do edifício. Desta forma, é possível garantir uma continuidade adequada quando elementos unidimensionais são conectados a elementos bidimensionais. 


\subsection{Acoplamento MEC/MEF}

É demonstrado, nesta Seção, como é feito o acoplamento entre as formulações do método dos elementos finitos (MEF) e do método dos elementos de contorno (MEC). A figura 5.8 representa o problema completo a ser estudado.

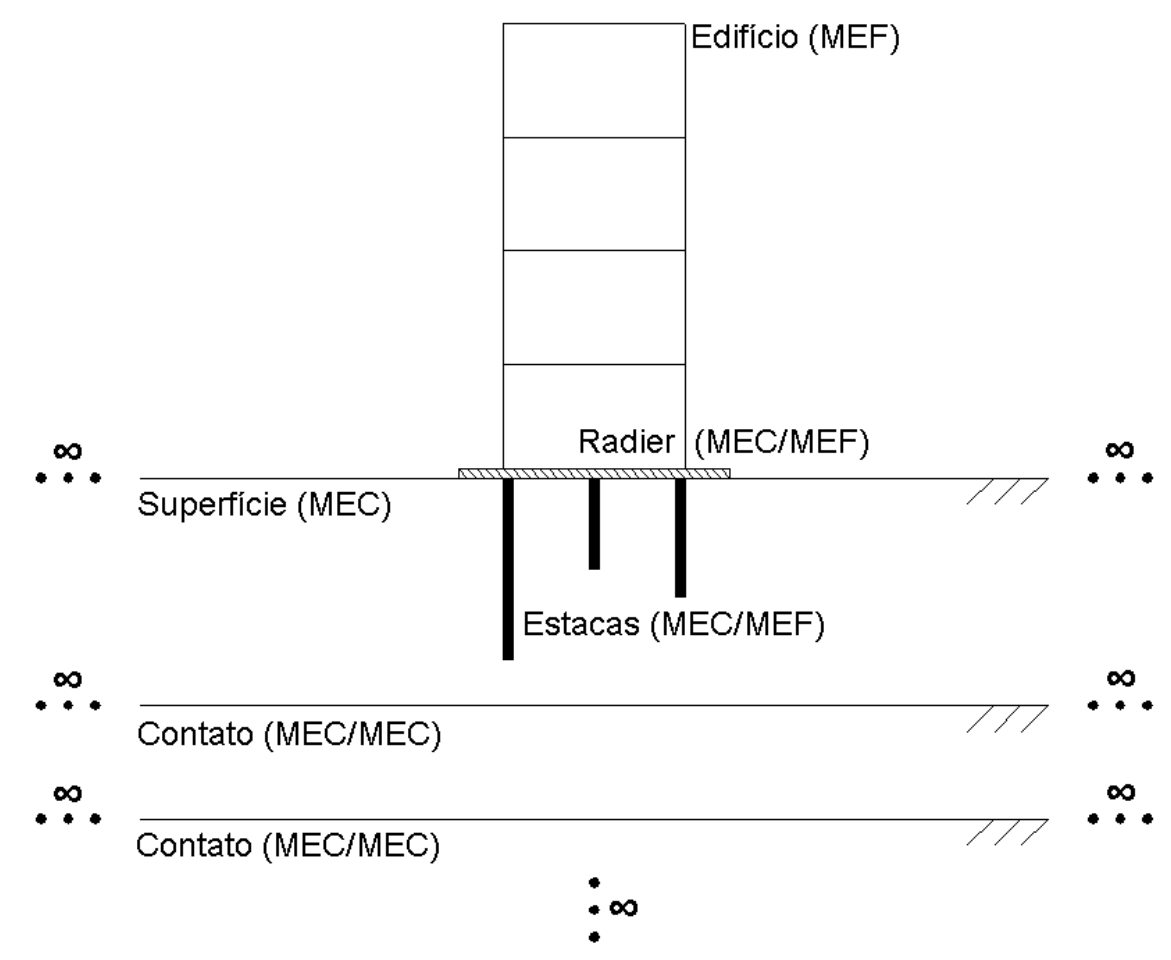

Figura 5.8: Problema de interação solo-estrutura

O edifício, o radier e as estacas são modeladas com o MEF, enquanto que o maciço não-homogêneo é simulado empregando o MEC. O maciço é representado por um semiespaço infinito nas direções radiais, e que pode também ser infinito na direção vertical dependendo das condições de contorno adotadas. A presença do radier é considerada no MEC como um conjunto de cargas aplicadas na superfície, enquanto que a presença das estacas é representada por linhas de carga aplicadas no interior do maciço. Como cada estaca é modelada por um único elemento finito considera-se, neste trabalho, que todas as estacas estão contidas na primeira camada do semi-espaço infinito.

Inicia-se escrevendo o sistema de equações do MEC que representa o semi-espaço infinito. Segundo as técnicas empregadas neste trabalho, a forma mais geral deste sistema 
é apresentada ao final do Capítulo 3, na expressão 3.54. Ou seja:

$$
\left[\begin{array}{ccc}
H_{l l} & H_{l c} & 0 \\
H_{c l} & H_{c c} & 0 \\
H_{i l} & H_{i c} & c_{i i}
\end{array}\right]\left\{\begin{array}{c}
u_{l} \\
u_{c} \\
u_{i}
\end{array}\right\}=\left[\begin{array}{cc}
G_{l l} & M_{l i} \\
G_{c l} & M_{c i} \\
G_{i l} & M_{i i}
\end{array}\right]\left\{\begin{array}{c}
p_{l} \\
s_{i}
\end{array}\right\}
$$

O índice $l$ se refere à superfície do maciço, incluindo o contato com o radier. O índice $c$ indica o conjunto de todas as superfícies de contato entre camadas e o índice $i$, por sua vez, se refere a termos calculados para pontos internos, ou seja, as estacas. Todas as matrizes do tipo $[H]$ e $[G]$ provém da integração dos contornos, enquanto que as matrizes do tipo $[M]$ provém da integração das linhas de carga que representam as estacas e a matriz $\left[c_{i i}\right]$ é calculada conforme apresentado no Capítulo 3. Os vetores do tipo $\{u\}$ contém deslocamentos, o vetor $\left\{p_{l}\right\}$ contém carregamentos aplicados na superfície do maciço e $\left\{s_{i}\right\}$ contém as cargas aplicadas nas linhas de carga.

Conforme pode ser observado em 5.79, a matriz do lado direito da igualdade não é quadrada. Isto ocorre devido à estratégia utilizada na técnica alternativa descrita no Capítulo 3, em que as cargas dos contatos entre camadas são eliminadas. Entretanto, para tornar possível a formulação empregada neste trabalho no acoplamento MEC/MEF, é necessário que esta matriz seja inversível. A solução aqui adotada é calcular, também para os contatos, as contribuições das cargas e posicioná-las na matriz de forma a torná-la quadrada. No entanto, este procedimento torna necessário que tais cargas sejam igualadas a zero. Isto é feito depois que as equações do MEC são agrupadas às do MEF, conforme descrito mais adiante. Seguindo com a dedução, mantendo os mesmos índices definidos anteriormente, a equação 5.79 se torna:

$$
\left[\begin{array}{ccc}
H_{l l} & H_{l c} & 0 \\
H_{c l} & H_{c c} & 0 \\
H_{i l} & H_{i c} & c_{i i}
\end{array}\right]\left\{\begin{array}{c}
u_{l} \\
u_{c} \\
u_{i}
\end{array}\right\}=\left[\begin{array}{ccc}
G_{l l} & G_{l c} & M_{l i} \\
G_{c l} & G_{c c} & M_{c i} \\
G_{i l} & G_{i c} & M_{i i}
\end{array}\right]\left\{\begin{array}{c}
p_{l} \\
p_{c} \\
s_{i}
\end{array}\right\}
$$

Para permitir a manipulação matricial necessária ao acoplamento MEC/MEF é preciso diferenciar, entre os nós que pertencem à superfície do maciço, os que participam do contato com o radier daqueles que não participam deste contato. Empregando o índice $r$ para os nós em contato com o radier e mantendo o índice $l$ somente para os que não 
pertencem a este contato, a igualdade 5.80 se torna:

$$
\left[\begin{array}{cccc}
H_{l l} & H_{l r} & H_{l c} & 0 \\
H_{r l} & H_{r r} & H_{r c} & 0 \\
H_{c l} & H_{c r} & H_{c c} & 0 \\
H_{i l} & H_{i r} & H_{i c} & c_{i i}
\end{array}\right]\left\{\begin{array}{c}
u_{l} \\
u_{r} \\
u_{c} \\
u_{i}
\end{array}\right\}=\left[\begin{array}{cccc}
G_{l l} & G_{l r} & G_{l c} & M_{l i} \\
G_{r l} & G_{r r} & G_{r c} & M_{r i} \\
G_{c l} & G_{c r} & G_{c c} & M_{c i} \\
G_{i l} & G_{i r} & G_{i c} & M_{i i}
\end{array}\right]\left\{\begin{array}{c}
p_{l} \\
p_{r} \\
p_{c} \\
s_{i}
\end{array}\right\}
$$

Como a matriz à direita da igualdade 5.81 é inversível, é possível isolar o vetor de cargas da seguinte forma:

$$
\left[\begin{array}{cccc}
G_{l l} & G_{l r} & G_{l c} & M_{l i} \\
G_{r l} & G_{r r} & G_{r c} & M_{r i} \\
G_{c l} & G_{c r} & G_{c c} & M_{c i} \\
G_{i l} & G_{i r} & G_{i c} & M_{i i}
\end{array}\right]^{-1}\left[\begin{array}{cccc}
H_{l l} & H_{l r} & H_{l c} & 0 \\
H_{r l} & H_{r r} & H_{r c} & 0 \\
H_{c l} & H_{c r} & H_{c c} & 0 \\
H_{i l} & H_{i r} & H_{i c} & c_{i i}
\end{array}\right]\left\{\begin{array}{c}
u_{l} \\
u_{r} \\
u_{c} \\
u_{i}
\end{array}\right\}=\left\{\begin{array}{c}
p_{l} \\
p_{r} \\
p_{c} \\
s_{i}
\end{array}\right\}
$$

Efetuando o produto das duas matrizes à esquerda da igualdade 5.82, obtém-se:

$$
\left[\begin{array}{cccc}
B_{l l} & B_{l r} & B_{l c} & B_{l i} \\
B_{r l} & B_{r r} & B_{r c} & B_{r i} \\
B_{c l} & B_{c r} & B_{c c} & B_{c i} \\
B_{i l} & B_{i r} & B_{i c} & B_{i i}
\end{array}\right]\left\{\begin{array}{c}
u_{l} \\
u_{r} \\
u_{c} \\
u_{i}
\end{array}\right\}=\left\{\begin{array}{c}
p_{l} \\
p_{r} \\
p_{c} \\
s_{i}
\end{array}\right\}
$$

A igualdade 5.83 é o ponto de partida da formulação do MEC para o acoplamento com o MEF. Entretanto, antes que as equações dos dois métodos sejam agrupadas, devese atentar à incompatibilidade existente entre as cargas do MEC e as do MEF. Esta incompatibilidade é ilustrada na figura 5.9 .
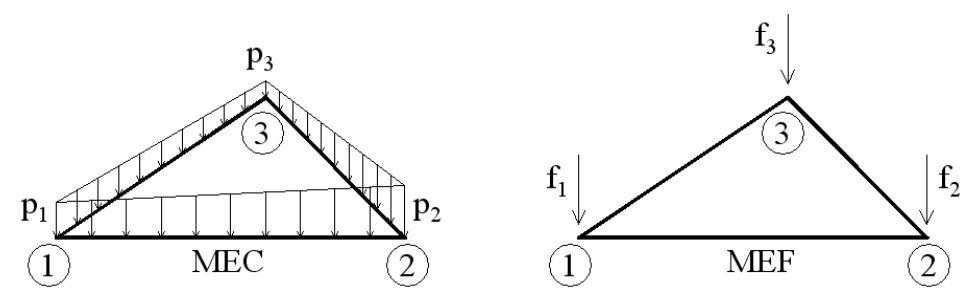

Figura 5.9: Incompatibilidade entre as cargas de superfície e as cargas nodais

Conforme apresentado na figura, as cargas do MEC são aplicadas de forma distribuída sobre os elementos, enquanto que as do MEF são aplicadas de forma concentrada nos nós. Portanto, para possibilitar que os métodos sejam coerentemente acoplados, a solução 
empregada neste trabalho é transformar as cargas distribuídas do MEC em cargas nodais concentradas, assim como no MEF. Também é possível transformar as cargas nodais concentradas do MEF em carregamentos distribuídos como no MEC, no entanto esta opção foi descartada porque, segundo Almeida (2003b), este procedimento exige maior esforço computacional.

O procedimento para transformar os carregamentos distribuídos em cargas nodais consiste, basicamente, em escrever a expressão de sua energia potencial total e então minimizar esta energia segundo os graus de liberdade definidos em cada nó. Este procedimento já foi realizado para as linhas de carga na Seção 5.3 , e o resultado foi uma expressão do tipo:

$$
\left\{r_{i}\right\}=\left[Q_{i i}\right]\left\{s_{i}\right\}
$$

em que $\left\{r_{i}\right\}$ é o vetor de cargas nodais e $\left[Q_{i i}\right]$ é uma matriz cujos índices foram deduzidos na Seção 5.3. Resta então escrever os carregamentos distribuídos sobre os elementos triangulares da superfície do maciço em termos de cargas nodais concentradas. Como o procedimento a ser realizado é o mesmo para as três direções do sistema de coordenadas global $x_{1} x_{2} x_{3}$, por questão de praticidade, nas próximas deduções é tomada uma única direção.

Parte-se do trabalho realizado pelas cargas de superfície. Este trabalho, analisado em um único elemento finito, pode ser representado pela expressão:

$$
T=\int_{A} p\left(\xi_{1}, \xi_{2}, \xi_{3}\right) w\left(\xi_{1}, \xi_{2}, \xi_{3}\right) d A
$$

em que $\xi_{1}$ e $\xi_{2}$ são coordenadas locais definidas no elemento e $\xi_{3}$ é função das outras duas. A função $p$ é uma aproximação das forças de superfície atuantes no elemento, e a função $w$ é uma aproximação do campo de deslocamentos do elemento. A área do elemento está indicada pela letra $A$.

Como na formulação do MEC apresentada no Capítulo 2 é considerada uma aproximação linear de forças e deslocamentos, conforme a figura 5.10, as aproximações de $w$ e $p$ são adotadas como lineares no cálculo do trabalho das cargas. Esta aproximação resulta nas funções:

$$
w\left(\xi_{1}, \xi_{2}, \xi_{3}\right)=w_{1} \xi_{1}+w_{2} \xi_{2}+w_{3} \xi_{3}
$$


e

$$
p\left(\xi_{1}, \xi_{2}, \xi_{3}\right)=p_{1} \xi_{1}+p_{2} \xi_{2}+p_{3} \xi_{3}
$$

com

$$
\xi_{3}=1-\xi_{1}-\xi_{2}
$$
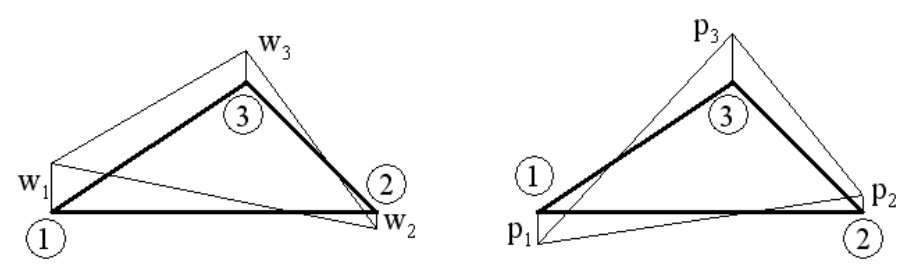

Figura 5.10: Aproximações lineares adotadas para $w$ e $p$

Substituindo as funções 5.86 e 5.87 na expressão 5.85, obtém-se a expressão:

$$
T=\int_{A}\left(w_{1} \xi_{1}+w_{2} \xi_{2}+w_{3} \xi_{3}\right)\left(p_{1} \xi_{1}+p_{2} \xi_{2}+p_{3} \xi_{3}\right) d A
$$

A expressão final do trabalho realizado pelas forças de superfície é obtida desenvolvendo-se a expressão 5.89 e efetuando-se a integral. Neste procedimento, uma ferramenta útil é a seguinte fórmula:

$$
\int_{A} \xi_{1}^{\eta 1} \xi_{2}^{\eta 2} \xi_{3}^{\eta 3} d A=2 A \frac{\eta_{1} ! \eta_{2} ! \eta_{3} !}{\left(\eta_{1}+\eta_{2}+\eta_{3}+2\right) !}
$$

Depois de chegar à expressão final do trabalho realizado pelas forças de superfície, deve-se minimizar o funcional da energia potencial acumulada no elemento finito. Ao final das deduções, chega-se à igualdade:

$$
\left\{\begin{array}{l}
r_{1} \\
r_{2} \\
r_{3}
\end{array}\right\}=\frac{A}{12}\left[\begin{array}{lll}
2 & 1 & 1 \\
1 & 2 & 1 \\
1 & 1 & 2
\end{array}\right]\left\{\begin{array}{l}
p_{1} \\
p_{2} \\
p_{3}
\end{array}\right\}
$$

O vetor à direita da igualdade em 5.91 contém o valor da carga distribuída nos nós de um elemento qualquer da superfície do solo, segundo uma direção qualquer. O vetor à esquerda contém as cargas nodais equivalentes, obtido por meio da matriz de transformação à direita. 
A partir da relação 5.91, é possível encontrar a influência de um único elemento de contorno no vetor de cargas nodais equivalente do MEF. Para relacionar todos os termos dos vetores, esta regra deve ser repetida para todos os elementos de contorno que compõem a malha da superfície do maciço. O resultado são as seguintes expressões:

$$
\begin{array}{r}
\left\{r_{l}\right\}=\left[Q_{l l}\right]\left\{p_{l}\right\} \\
\left\{r_{r}\right\}=\left[Q_{r r}\right]\left\{p_{r}\right\} \\
\left\{r_{c}\right\}=\left[Q_{c c}\right]\left\{p_{c}\right\}
\end{array}
$$

É possível combinar as expressões $5.84,5.92,5.93$ e 5.94 da seguinte forma:

$$
\left\{\begin{array}{c}
r_{l} \\
r_{r} \\
r_{c} \\
r_{i}
\end{array}\right\}=\left[\begin{array}{cccc}
Q_{l l} & 0 & 0 & 0 \\
0 & Q_{r r} & 0 & 0 \\
0 & 0 & Q_{c c} & 0 \\
0 & 0 & 0 & Q_{i i}
\end{array}\right]\left\{\begin{array}{c}
p_{l} \\
p_{r} \\
p_{c} \\
s_{i}
\end{array}\right\}
$$

Observa-se, na igualdade 5.95, que as reações são independentes entre si. Isto acontece porque são empregados nós duplos nos pontos em comum entre malhas, tornado-as todas independentes. Esta propriedade é útil para que a contribuição das cargas de contato $\left\{p_{c}\right\}$ possa ser posteriormente igualada a zero, pois isto será feito igualando-se $\left\{r_{c}\right\}$ a zero.

Assim, para transformar as cargas do MEC em forças concentradas nos nós, basta multiplicar ambos os lados da equação 5.83 pela matriz apresentada na expressão 5.95 . Este procedimento fornece a seguinte relação:

$$
\left[\begin{array}{cccc}
Q_{l l} & 0 & 0 & 0 \\
0 & Q_{r r} & 0 & 0 \\
0 & 0 & Q_{c c} & 0 \\
0 & 0 & 0 & Q_{i i}
\end{array}\right]\left[\begin{array}{cccc}
B_{l l} & B_{l r} & B_{l c} & B_{l i} \\
B_{r l} & B_{r r} & B_{r c} & B_{r i} \\
B_{c l} & B_{c r} & B_{c c} & B_{c i} \\
B_{i l} & B_{i r} & B_{i c} & B_{i i}
\end{array}\right]\left\{\begin{array}{c}
u_{l} \\
u_{r} \\
u_{c} \\
u_{i}
\end{array}\right\}=\left[\begin{array}{cccc}
Q_{l l} & 0 & 0 & 0 \\
0 & Q_{r r} & 0 & 0 \\
0 & 0 & Q_{c c} & 0 \\
0 & 0 & 0 & Q_{i i}
\end{array}\right]\left\{\begin{array}{c}
p_{l} \\
p_{r} \\
p_{c} \\
s_{i}
\end{array}\right\}
$$


e, por fim:

$$
\left[\begin{array}{cccc}
D_{l l} & D_{l r} & D_{l c} & D_{l i} \\
D_{r l} & D_{r r} & D_{r c} & D_{r i} \\
D_{c l} & D_{c r} & D_{c c} & D_{c i} \\
D_{i l} & D_{i r} & D_{i c} & D_{i i}
\end{array}\right]\left\{\begin{array}{c}
u_{l} \\
u_{r} \\
u_{c} \\
u_{i}
\end{array}\right\}=\left\{\begin{array}{c}
r_{l} \\
r_{r} \\
r_{c} \\
r_{i}
\end{array}\right\}
$$

A igualdade 5.97 representa toda a contribuição do MEC no acoplamento, com os carregamentos expressos como cargas concentradas nos nós. O passo seguinte é analisar as contribuições do MEF, que podem ser equacionadas como segue:

$$
[K]\{u\}=\{f\}-\{r\}
$$

Em 5.98, $[K]$ é a matriz de rigidez que representa todos os elementos finitos definidos na simulação. Os vetores $\{u\}$ e $\{f\}$ contém, respectivamente, todos os deslocamentos e todas as cargas prescritas no MEF. O vetor $\{r\}$ contém as cargas provenientes do MEC, que aparecem com o sinal negativo por serem tratadas como reativas na equação. Na sequência, esta equação deve ser reescrita para que possam ser visualizados os trechos referentes ao edifício, ao radier e às estacas, conforme ilustrado na figura 5.8. Assim:

$$
\left[\begin{array}{ccc}
K_{e e} & K_{e r} & K_{e i} \\
K_{r e} & K_{r r} & K_{r i} \\
K_{i e} & K_{i r} & K_{i i}
\end{array}\right]\left\{\begin{array}{l}
u_{e} \\
u_{r} \\
u_{i}
\end{array}\right\}=\left\{\begin{array}{c}
f_{e} \\
f_{r} \\
f_{i}
\end{array}\right\}-\left\{\begin{array}{c}
r_{e} \\
r_{r} \\
r_{i}
\end{array}\right\}
$$

Os índices $r$ e $i$ empregados na equação 5.99 têm o mesmo significado definido anteriormente na igualdade 5.97. O índice $e$, por sua vez, se refere a nós que não estão em contato com o MEC. A etapa seguinte é expandir as matrizes e vetores de 5.99 de forma a incluir os nós do MEC que não estão em contato com o MEF. O resultado é:

$$
\left[\begin{array}{ccccc}
K_{e e} & K_{e r} & 0 & 0 & K_{e i} \\
K_{r e} & K_{r r} & 0 & 0 & K_{r i} \\
0 & 0 & 0 & 0 & 0 \\
0 & 0 & 0 & 0 & 0 \\
K_{i e} & K_{i r} & 0 & 0 & K_{i i}
\end{array}\right]\left\{\begin{array}{c}
u_{e} \\
u_{r} \\
u_{l} \\
u_{c} \\
u_{i}
\end{array}\right\}=\left\{\begin{array}{c}
f_{e} \\
f_{r} \\
f_{l} \\
f_{c} \\
f_{i}
\end{array}\right\}-\left\{\begin{array}{c}
r_{e} \\
r_{r} \\
r_{l} \\
r_{c} \\
r_{i}
\end{array}\right\}
$$

Todos os índices utilizados na equação 5.100 foram definidos anteriomente. Observa-se 
que na terceira e quarta linhas de 5.100 estão estabelecidas as seguintes identidades:

$$
\left\{f_{l}\right\}=\left\{r_{l}\right\}, \quad\left\{f_{c}\right\}=\left\{r_{c}\right\}
$$

Isto significa que as reações do solo referentes aos pontos que não têm contato com o MEF são as próprias cargas prescritas. Desta forma, caso seja necessário considerar algum carregamento aplicado na superfície livre e que não faça parte do contato, basta prescrevê-lo em $\left\{f_{l}\right\}$. Se torna possível também igualar a zero as cargas de contato entre camadas, procedimento necessário devido à estratégia empregada para tornar quadradas as matrizes da equação 5.80. Igualando $\left\{f_{c}\right\}$ a zero as reações $\left\{r_{c}\right\}$ automaticamente são igualadas a zero, conforme apresentado em 5.101. Sendo $\left\{r_{c}\right\}$ igual a zero todas as cargas de contato $\left\{p_{c}\right\}$ também se tornam zero, devido à relação 5.94. Portanto, basta igualar sempre o vetor $\left\{f_{c}\right\}$ a zero para resolver o problema criado na relação 5.80 .

Em seguida, reescreve-se a igualdade 5.97 de forma a incluir os nós do MEF que não fazem parte do contato com o MEC. Ou seja:

$$
\left[\begin{array}{ccccc}
0 & 0 & 0 & 0 & 0 \\
0 & D_{r r} & D_{r l} & D_{r c} & D_{r i} \\
0 & D_{l r} & D_{l l} & D_{l c} & D_{l i} \\
0 & D_{c r} & D_{c l} & D_{c c} & D_{c i} \\
0 & D_{i r} & D_{i l} & D_{i c} & D_{i i}
\end{array}\right]\left\{\begin{array}{c}
u_{e} \\
u_{r} \\
u_{l} \\
u_{c} \\
u_{i}
\end{array}\right\}=\left\{\begin{array}{c}
r_{e} \\
r_{r} \\
r_{l} \\
r_{c} \\
r_{i}
\end{array}\right\}
$$

Observa-se que na primeira linha de 5.102 as reações $\left\{r_{e}\right\}$ são sempre igualadas a zero. Isto acontece porque o MEC não entra em contato com estes nós.

A etapa final é substituir a expressão 5.102 na relação 5.100 , agrupando todas as equações do acoplamento MEC/MEF. Este procedimento fornece:

$$
\left[\begin{array}{ccccc}
K_{e e} & K_{e r} & 0 & 0 & K_{e i} \\
K_{r e} & K_{r r} & 0 & 0 & K_{r i} \\
0 & 0 & 0 & 0 & 0 \\
0 & 0 & 0 & 0 & 0 \\
K_{i e} & K_{i r} & 0 & 0 & K_{i i}
\end{array}\right]\left\{\begin{array}{c}
u_{e} \\
u_{r} \\
u_{l} \\
u_{c} \\
u_{i}
\end{array}\right\}=\left\{\begin{array}{c}
f_{e} \\
f_{r} \\
f_{l} \\
f_{c} \\
f_{i}
\end{array}\right\}-\left[\begin{array}{ccccc}
0 & 0 & 0 & 0 & 0 \\
0 & D_{r r} & D_{r l} & D_{r c} & D_{r i} \\
0 & D_{l r} & D_{l l} & D_{l c} & D_{l i} \\
0 & D_{c r} & D_{c l} & D_{c c} & D_{c i} \\
0 & D_{i r} & D_{i l} & D_{i c} & D_{i i}
\end{array}\right]\left\{\begin{array}{c}
u_{e} \\
u_{r} \\
u_{l} \\
u_{c} \\
u_{i}
\end{array}\right\}
$$

Na relação 5.103 , que representa o acoplamento MEC/MEF, o número de incógnitas é 
igual ao número de equações. Além disto foram eliminadas todas as reações desconhecidas do solo, restando apenas o vetor de deslocamentos como icógnita. Como tanto a matriz à esquerda da igualdade como a matriz à direita da igualdade multiplicam este mesmo vetor, torna-se possível separar-se as incógnitas dos valores prescritos. O resutado é:

$$
\left[\begin{array}{ccccc}
K_{e e} & K_{e r} & 0 & 0 & K_{e i} \\
K_{r e} & \left(K_{r r}+D_{r r}\right) & D_{r l} & D_{r c} & \left(K_{r i}+D_{r i}\right) \\
0 & D_{l r} & D_{l l} & D_{l c} & D_{l i} \\
0 & D_{c r} & D_{c l} & D_{c c} & D_{c i} \\
K_{i e} & \left(K_{i r}+D_{i r}\right) & D_{i l} & D_{i c} & \left(K_{i i}+D_{i i}\right)
\end{array}\right]\left\{\begin{array}{c}
u_{e} \\
u_{r} \\
u_{l} \\
u_{c} \\
u_{i}
\end{array}\right\}=\left\{\begin{array}{c}
f_{e} \\
f_{r} \\
f_{l} \\
0 \\
f_{i}
\end{array}\right\}
$$

ou simplesmente

$$
[A]\{u\}=\{f\}
$$

A igualdade 5.105 é um sistema de equações lineares, no qual o número de incógnitas é igual ao número de equações. Desta forma, é possível resolver o sistema e obter todos os deslocamentos incógnitos do acoplamento MEC/MEF. As forças prescritas nos contatos entre camadas devem ser sempre iguais a zero, tal como indicado no vetor à direita da igualdade 5.104 .

Podem ser empregadas, na equação 5.105, técnicas usuais do MEF caso se queira considerar uma superfície indeslocável no solo ou qualquer outro tipo de restrição de deslocamento. O procedimento adotado neste trabalho inicia-se zerando os termos do vetor $\{f\}$ referentes aos deslocamentos restringidos. Em seguida, as linhas e colunas correspondentes da matriz $[A]$ são zeradas e, por fim, iguala-se a 1 os termos correspondentes da diagonal principal de $[A]$.

Calculados os deslocamentos, torna-se possível determinar vários outros valores de interesse tanto no MEC como no MEF. Com o exemplo, cita-se a deteminação de esforços internos nos elementos estruturais do edifício e o cálculo de valores em pontos internos do maciço de solos.

\subsection{Considerações finais}

Neste Capítulo foi apresentada de forma sucinta a formulação do método dos elementos finitos (MEF), bem como a estratégia empregada para acoplar esta formulação ao método 
dos elementos de contorno (MEC).

Aplicando a teoria advinda da Mecânica dos Sólidos, foi obtida uma equação de equilíbrio válida para um sólido qualquer. A partir desta equação e de técnicas de resíduos ponderados, foi possível obter uma expressão que representa os trabalhos externo e interno do sólido e que compõem sua energia potencial total. Dividindo o domínio em um número qualquer de subdomínios denominados elementos finitos aplicou-se, a cada um deles, a equação da energia previamente obtida. Adotando aproximações para os deslocamentos e deformações e minimizando a energia potencial total, chegou-se a uma matriz de rigidez e a um vetor de cargas nodais para cada elemento finito. A partir destes foi possível montar então uma matriz e um vetor globais, ou seja, válidos para toda a estrutura. Esta matriz e este vetor são utilizados para montar um sistema de equações global, ao qual devem ser aplicadas as condições de contorno do problema. Após aplicar estas condições e resolver o sistema, obtém-se os deslocamentos incógnitos do sólido em questão.

Ao final do Capítulo, apresentou-se a teoria que será empregada para acoplar a formulação do MEF com a do MEC. Antes de agrupar as equações é necessário transformar as forças de superfície do MEC em cargas nodais equivalentes, para que possam ser posteriormente aplicadas como forças reativas na expressão do MEF. Foi necessário adaptar as manipulações matriciais à técnica alternativa de subregiões do MEC, descrita no Capítulo 3. a qual pode gerar matrizes que não são quadradas. 


\section{Capítulo 6}

\section{Exemplos}

\subsection{Introdução}

Neste Capítulo são apresentadas as principais aplicações da formulação desenvolvida neste trabalho, cujo objetivo é a simulação de problemas de interação solo-estrutura. É empregado o método dos elementos de contorno (MEC) para simular o maciço de solos, conforme a teoria apresentada nos Capítulos 2, 3 e 4. A estrutura, por sua vez, é modelada empregando o método dos elementos finitos (MEF), conforme apresentado no Capítulo 5. Também no Capítulo 5 é descrito como é feito o acoplamento do MEF com o MEC, permitindo que estruturas modeladas pelo MEF interajam com o solo simulado pelo MEC. Todas as análises são estáticas, e os materiais são considerados isotrópicos e elástico-lineares.

As malhas de ECs, ECIs e EFs são escolhidas seguindo o mesmo critério adotado nos exemplos do Capítulo 4. Desta forma, a distância a partir da qual os ECIs são empregados é determinada em um estudo realizado utilizando malhas pouco refinadas, no qual verificase a partir de qual distância o resultado não é mais alterado de forma significativa. Depois de encontrada e fixada tal distância, parte-se para um segundo estudo no qual é variado somente o refinamento da malha. O refinamento final é então determinado quando verificase que um aumento no número de graus de liberdade não altera os resultados de forma significativa, consistindo na convergência para o valor final a ser apresentado no exemplo em questão.

Na Seção 6.2 são apresentados exemplos relacionados à interação de estacas com o solo. A Seção 6.3. por sua vez, contém exemplos de interação placa-solo. Na sequência, a 
Seção 6.4 apresenta exemplos que envolvem a interação de placas estaqueadas com o solo e, finalmente, a Seção 6.5 contém um exemplo que descreve a interação de um edifício com o solo, incluindo suas estruras de fundação.

\subsection{Interação de estacas com o solo}

\subsubsection{Estaca imersa em um semi-espaço infinito}

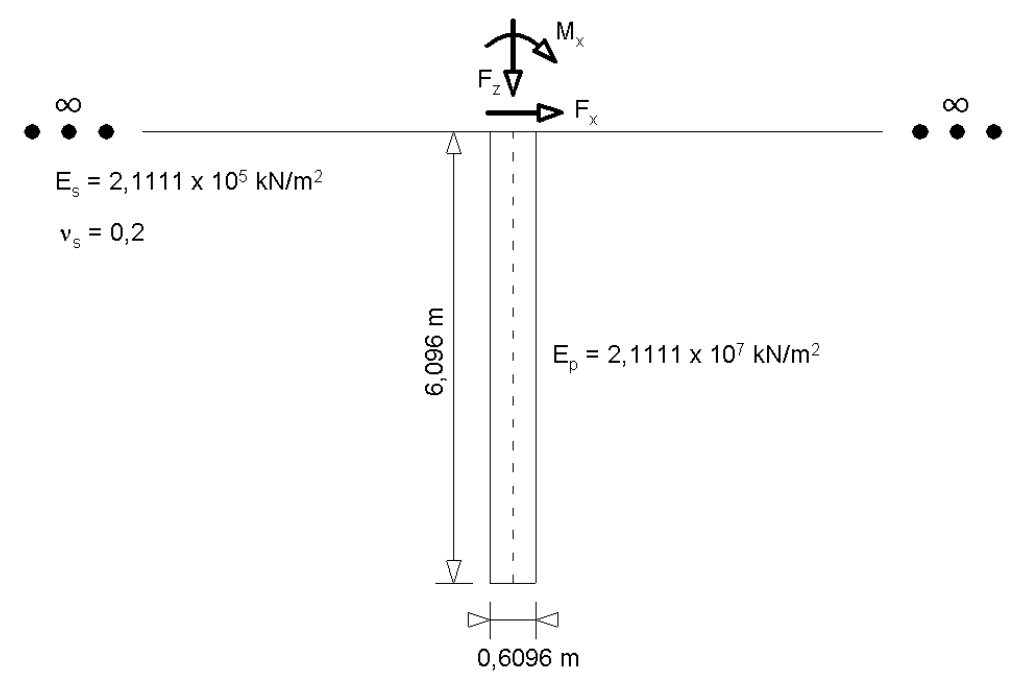

Figura 6.1: Estaca imersa no solo

Considera-se uma estaca cilíndrica e vertical imersa no solo, conforme ilustrado na figura 6.1. O solo é representado por um semi-espaço infinito, homogêneo, isotrópico e elástico-linear, com módulo de elasticidade de $2,1111 \times 10^{5} \mathrm{kN} / \mathrm{m}^{2}$ e coeficiente de Poisson de 0,2. A estaca, por sua vez, tem 0,6096 $m$ de diâmetro, 6,096 $m$ de comprimento e módulo de elasticidade igual a $2,1111 \times 10^{7} \mathrm{kN} / \mathrm{m}^{2}$.

São considerados três tipos de carregamento, aplicados um por vez no topo da estaca. O primeiro é uma força horizontal de $181,6 k N$, a qual é indicada na figura 6.1 como $F_{x}$. A segunda é o momento $M_{x}$, o qual está contido no plano da figura 6.1 e tem um valor de 95, $826 \mathrm{kNm}$. Por fim, considera-se uma força vertical de 726, $4 k N$, indicada em 6.1 como $F_{z}$.

A figura 6.2 apresenta a malha de elementos de contorno (EC) e elementos de contorno infinitos (ECI) empregada para simular a superfície do solo. Esta malha é composta por 56 ECs e 16 ECIs, e em seu centro está a origem do sistema de coordenadas globais. A estaca, por sua vez, é simulada empregando um único elemento finito (EF) com 14 


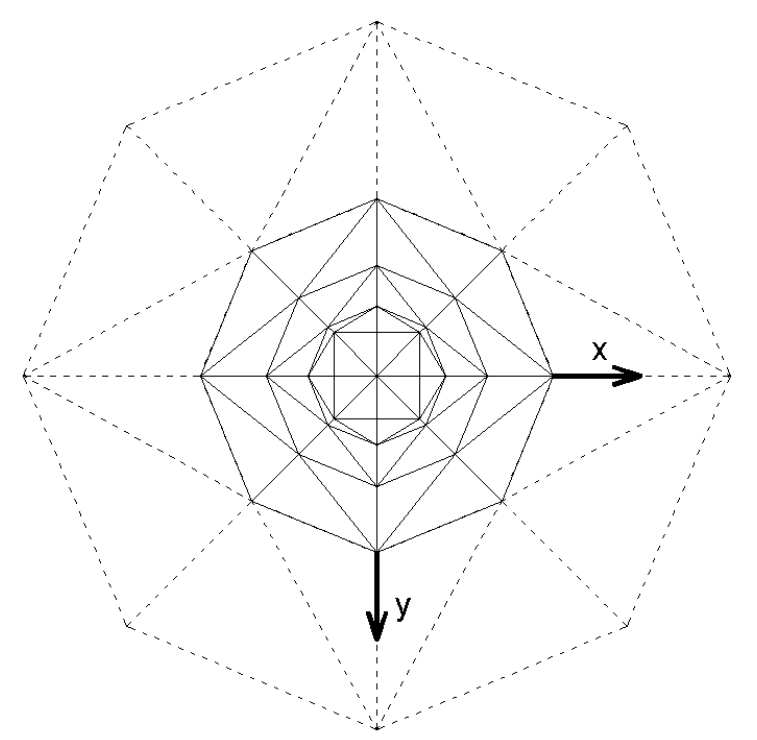

Figura 6.2: Malha de EC/ECI empregada

parâmetros nodais. A teoria que define este EF é apresentada em detalhes na Seção 5.3 . O topo da estaca está posicionado na origem do sistema de coordenadas, e seu eixo é perpendicular ao plano da malha do solo.

Os deslocamentos calculados ao longo do eixo da estaca foram organizados nos gráficos das figuras 6.3, 6.4 e 6.5, nos quais são comparados com os resultados fornecidos no trabalho de Filho et al. (2005).

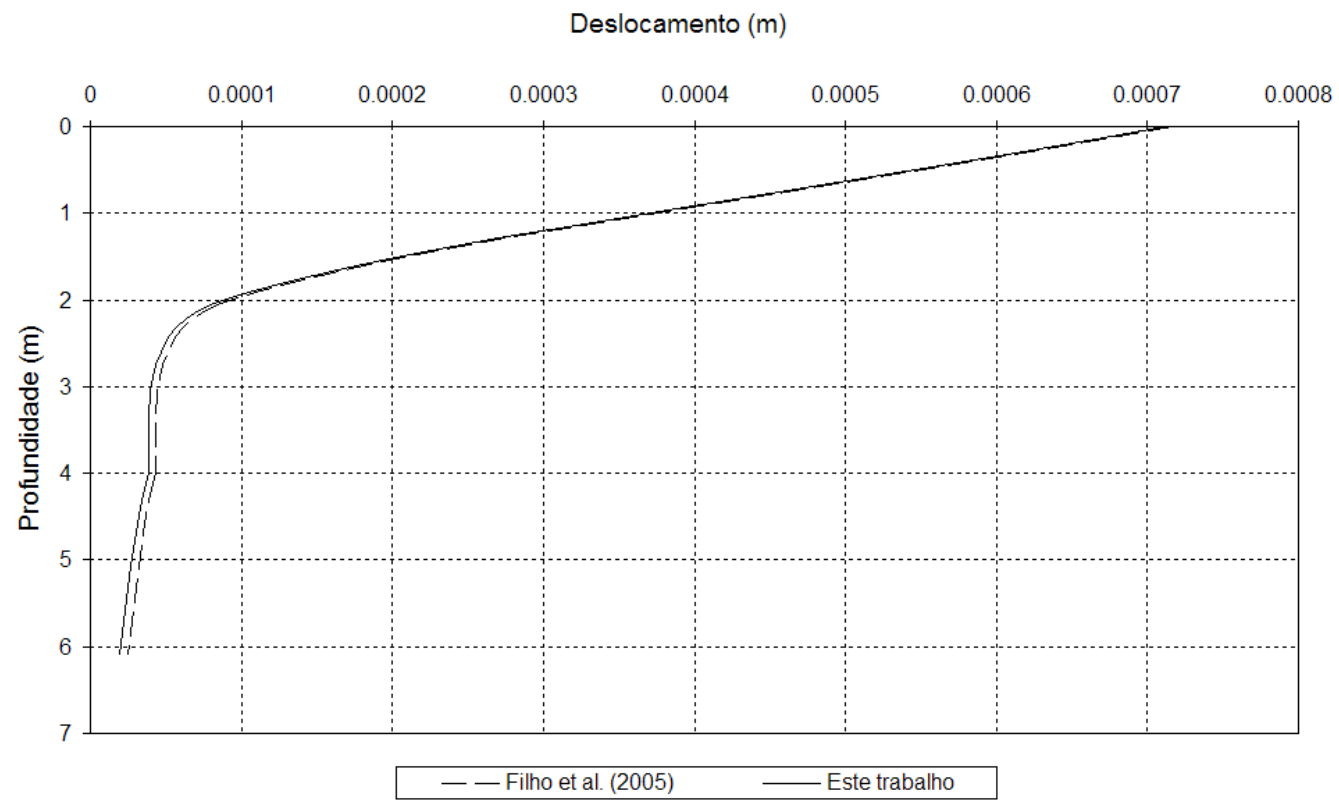

Figura 6.3: Deslocamento horizontal devido à carga de 181, $6 k N$

Desta forma, na figura 6.3 são apresentados os deslocamentos horizontais do eixo da 
estaca resultantes da carga horizontal de $181,6 \mathrm{kN}$ aplicada em seu topo. É possível observar que há grande concordância com os valores de Filho et al. (2005), sendo as duas curvas quase coincidentes.

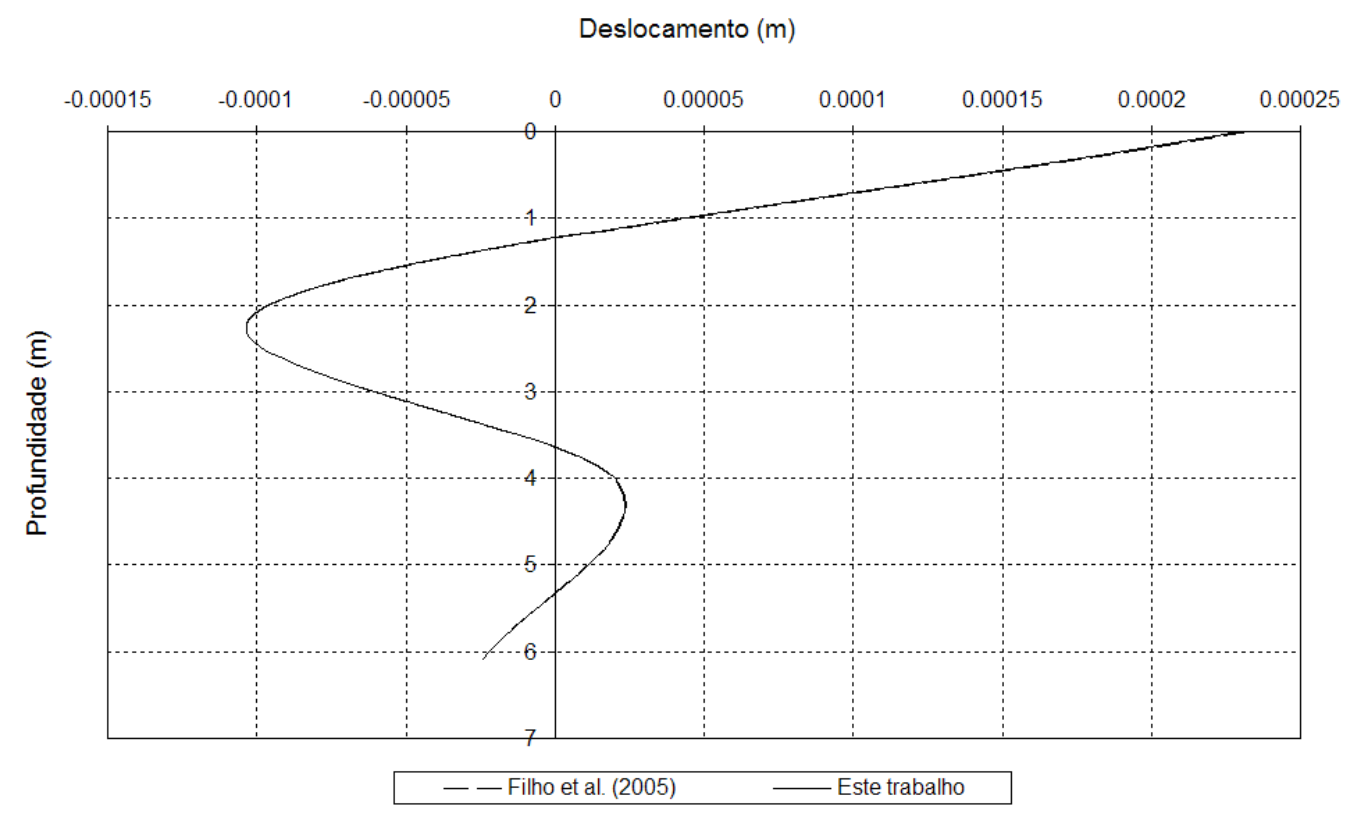

Figura 6.4: Deslocamento horizontal devido ao momento de 95, $826 \mathrm{kNm}$

Na figura 6.4. por outro lado, são apresentados os deslocamentos horizontais do eixo da estaca resultantes do momento fletor de 95, $826 \mathrm{kNm}$ aplicado em seu topo. Observase novamente grande concordância com os resultados de Filho et al. (2005), sendo difícil distinguir as duas curvas.

Por fim, na figura 6.5 são apresentados os deslocamentos verticais do eixo da estaca resultantes da força vertical de 726, $4 \mathrm{kN}$ aplicada no topo. Neste último gráfico foram colocados, além dos valores de Filho et al. (2005) e dos resultados obtidos com a malha de EC/ECI da figura 6.2, mais uma curva que foi montada com valores calculados com a mesma malha apresentada na figura 6.2 mas sem os ECIs. É possível observar grande concordância com Filho et al. (2005) quando os ECIs são empregados, mas por outro lado os resutados se tornam mais distantes sem os ECIs. Isto demonstra que a utilização dos ECIs tem contribuição positiva na precisão dos resultados.

\subsubsection{Nove estacas imersas em um semi-espaço infinito}

Neste exemplo é analisada a interação do solo com nove estacas cilíndricas e verticais, conforme esquematizado na figura 6.6. As estacas estão espaçadas de 1,5 m, têm módulo 


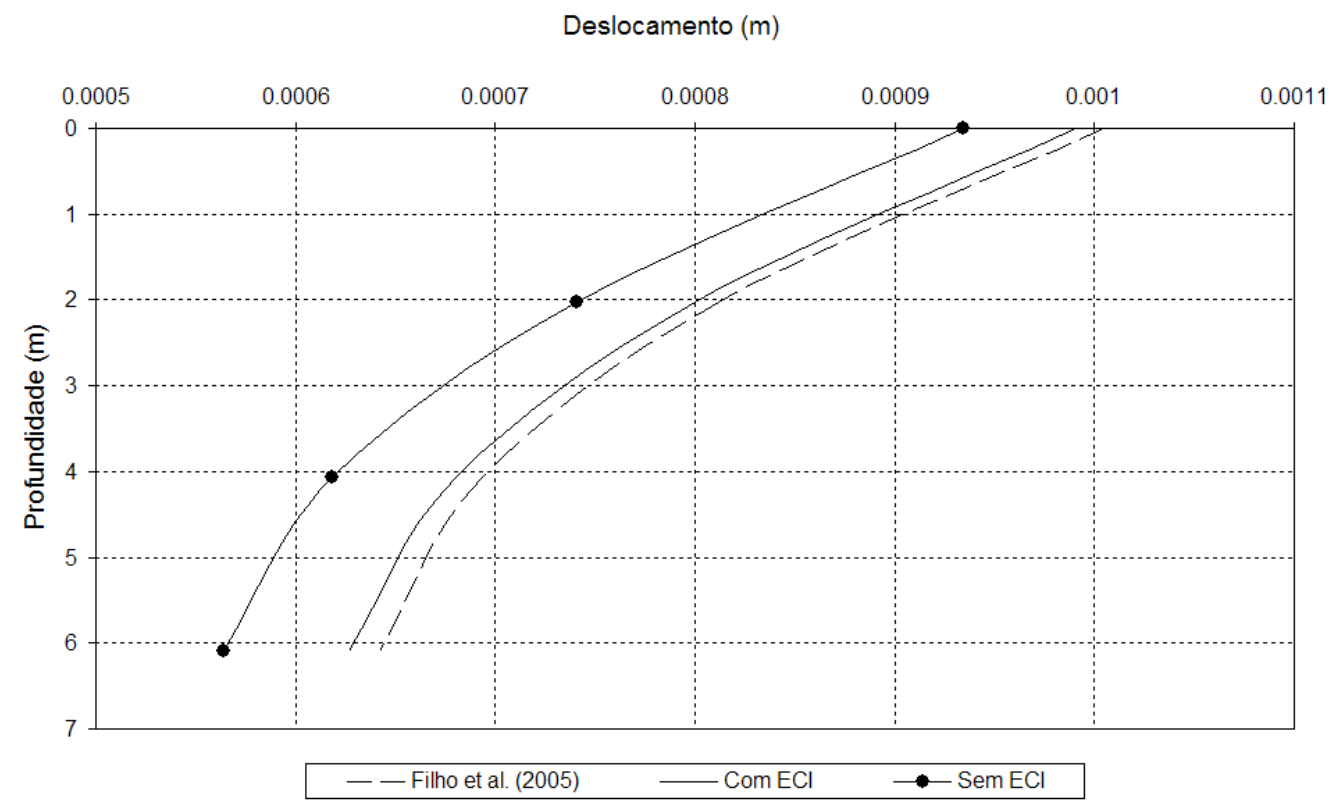

Figura 6.5: Deslocamento vertical devido à carga de 726, $4 \mathrm{kN}$

de elasticidade igual a $10^{7} \mathrm{kN} / \mathrm{m}^{2}$, diâmetro de $0,35 \mathrm{~m}$ e comprimento de $15 \mathrm{~m}$. No topo de cada estaca é aplicada uma força horizontal de $20 k N$, todas com a mesma direção. Devido à simetria do problema espera-se resultados equivalentes em algumas estacas, desta forma elas foram numeradas de 1 a 4 segundo esta equivalência. O solo é representado por um semi-espaço infinito com módulo de elasticidade de $10^{3} \mathrm{kN} / \mathrm{m}^{2}$ e coeficiente de Poisson de 0,2 .

A figura 6.7 apresenta a malha de elementos de contorno (EC) e elementos de contorno infinitos (ECI) utilizada para simular este problema. Os círculos destacados na região central da malha indicam a posição em planta das nove estacas. Esta malha é composta por 32 ECIs, posicionados nas bordas da malha, e 96 ECs. As estacas são simuladas com o método dos elementos finitos (MEF), empregando o elemento com 14 parâmetros nodais definido na Seção 5.3. O eixo das estacas é perpendicular ao plano da malha do solo.

Foi calculado o deslocamento horizontal, na direção das cargas aplicadas, do topo das estacas numeradas de 1 a 4 . Os resultados obtidos foram comparados com os de Filho et al. (2005), conforme apresentado na tabela 6.1.

Os valores obtidos apresentaram grande concordância com os de Filho et al. (2005) com erros iguais ou inferiores a $1 \%$, conforme pode se observado na tabela. Isto demonstra que a formulação desenvolvida neste trabalho tem precisão também em exemplos nos quais há mais de uma estaca a ser considerada. É interessante analisar também o deslocamento 


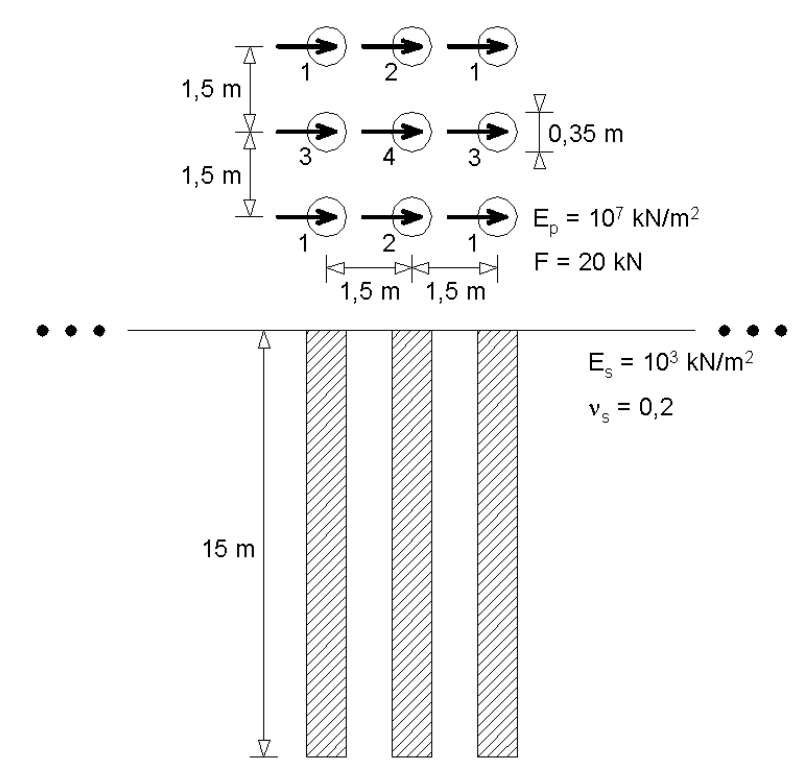

Figura 6.6: Nove estacas imersas no solo

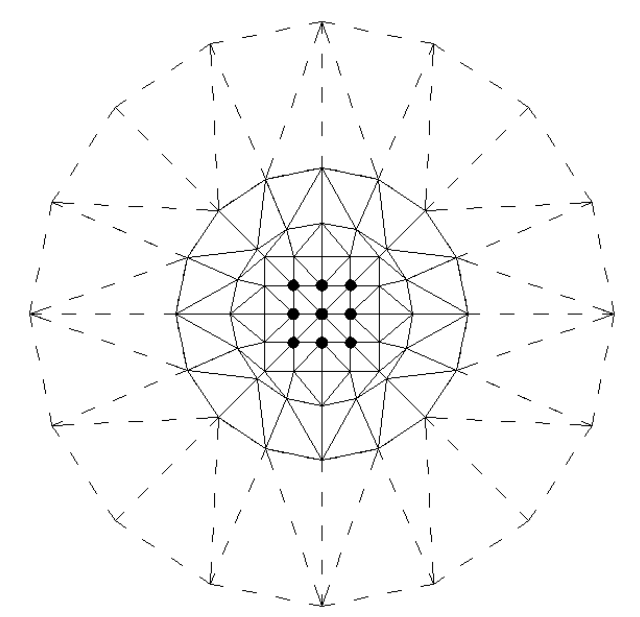

Figura 6.7: Malha de EC/ECI empregada

ao longo de todo o eixo de uma das estacas. Desta forma, na figura 6.8 é apresentado o deslocamento horizontal da estaca central na direção das cargas aplicadas.

Observa-se, mais uma vez, que os resultados apresentam boa concordância com os de Filho et al. (2005). Isto indica a eficiência da formulação apresentada neste trabalho.

\subsubsection{Nove estacas imersas em uma camada finita}

É analisada neste exemplo a interação de nove estacas cilíndricas com uma camada finita de solo, conforme ilustrado na figura 6.9. As estacas estão espaçadas de $4 m$, têm módulo de elasticidade igual a $2 \times 10^{7} \mathrm{kN} / \mathrm{m}^{2}$, diâmetro de $1,273 \mathrm{~m}$ e comprimento de 20 $m$. No topo de cada estaca é aplicada uma força vertical de $100 k N$. Devido à simetria do 


\begin{tabular}{|c|c|c|c|}
\hline Estaca & Este trabalho & Filho et al. (2005) & Erro (\%) \\
\hline 1 & 3,3278 & 3,2946 & 1,0 \\
\hline 2 & 3,5622 & 3,5284 & 1,0 \\
\hline 3 & 3,6162 & 3,5842 & 0,9 \\
\hline 4 & 3,8907 & 3,8632 & 0,7 \\
\hline
\end{tabular}

Tabela 6.1: Deslocamento horizontal no topo $\left(m \times 10^{-2}\right)$

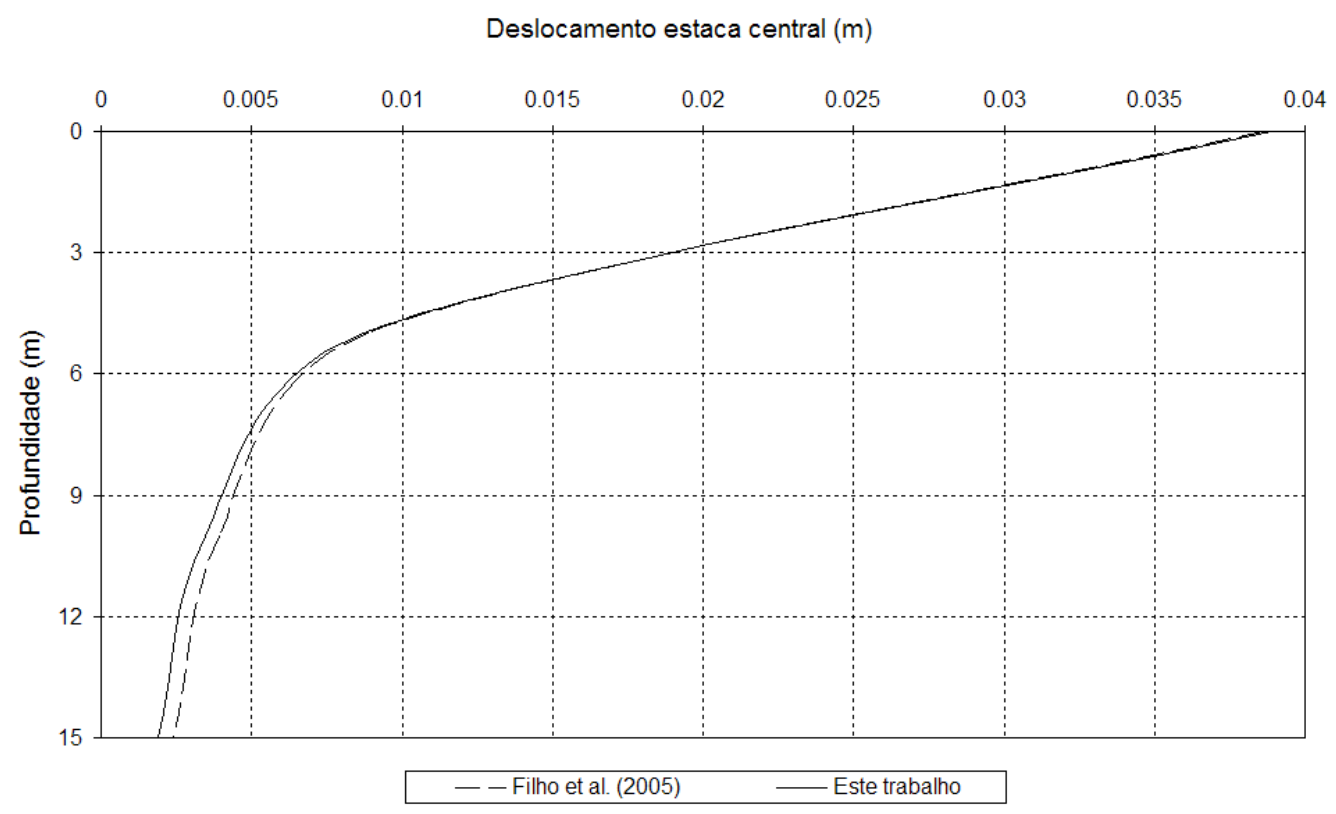

Figura 6.8: Deslocamento horizontal da estaca central

problema espera-se resultados equivalentes em algumas estacas, desta forma elas foram numeradas de 1 a 3 segundo esta equivalência. A camada de solo é representada como um meio infinito nas direções radiais, com $30 \mathrm{~m}$ de espessura, módulo de elasticidade de $2,5 \times 10^{4} \mathrm{kN} / \mathrm{m}^{2}$ e coeficiente de Poisson de 0,45 .

A figura 6.10 apresenta a malha de elementos de contorno (EC) e elementos de contorno infinitos (ECI) utilizada na superfície livre do solo e na base rígida, totalizando 192 ECs e 64 ECIs. Os círculos destacados na região central da malha indicam a posição do topo das estacas em relação à malha da superfície. As estacas são simuladas com o método dos elementos finitos (MEF), empregando o elemento com 14 parâmetros nodais definido na Seção 5.3. O eixo das estacas é perpendicular ao plano da malha do solo.

Na figura 6.11 é apresentado o deslocamento vertical da estaca central, juntamente com uma interpolação linear dos resultados obtidos por outros autores para o topo e base das estacas. Em Almeida e Paiva (2007) e Ottaviani (1975) foram adotadas estacas com seção transversal quadrada de $1 m$ de aresta, sendo a área total de fuste igual à das 


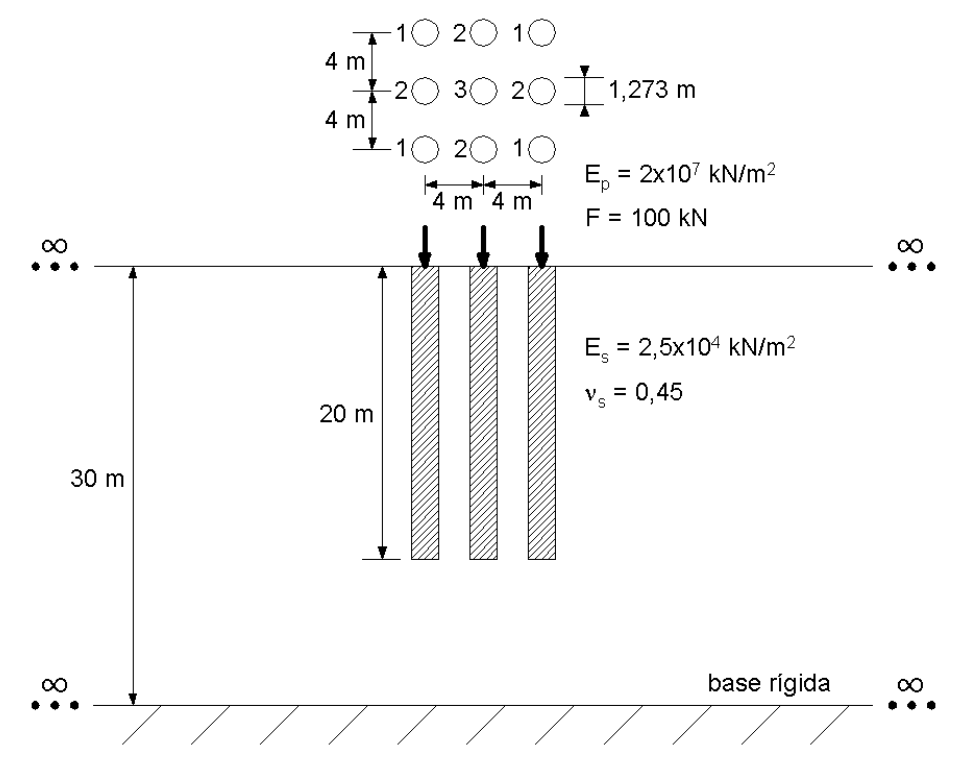

Figura 6.9: Nove estacas imersas na camada

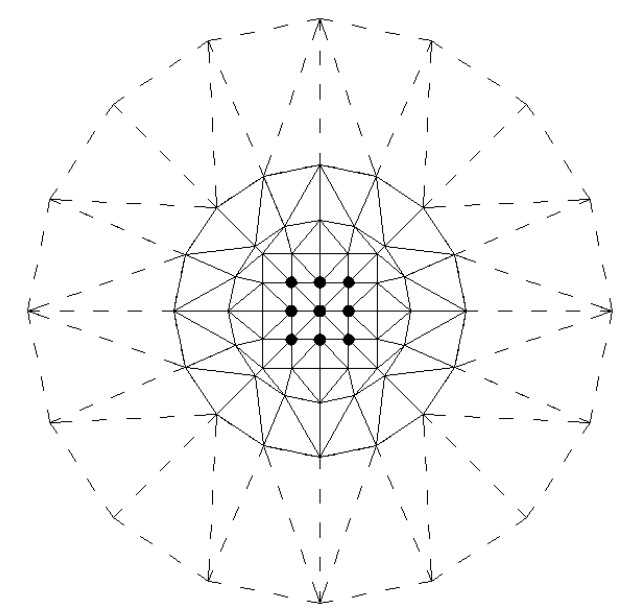

Figura 6.10: Malha de EC/ECI empregada

estacas cilíndricas apresentadas na figura 6.9. Segundo Ottaviani (1975), que comparou valores obtidos com seções transversais circulares e quadradas, estas duas aproximações produzem resultados equivalentes.

Conforme pode ser observado na figura 6.11, os deslocamentos obtidos por Ottaviani (1975) foram inferiores aos das demais formulações. Isto ocorre porque Ottaviani (1975) simula o solo empregando uma malha de elementos finitos tridimensionais que é finita nas direções radiais, se extendendo até uma distância de apenas $13 m$ das estacas numeradas como 1 e estando com os deslocamentos verticais restringidos nestes limites. Desta forma, o resultado no centro recebe uma influência considerável das vinculações impostas nos limites. A formulação de Almeida e Paiva (2007), por outro lado, por empregar elementos 


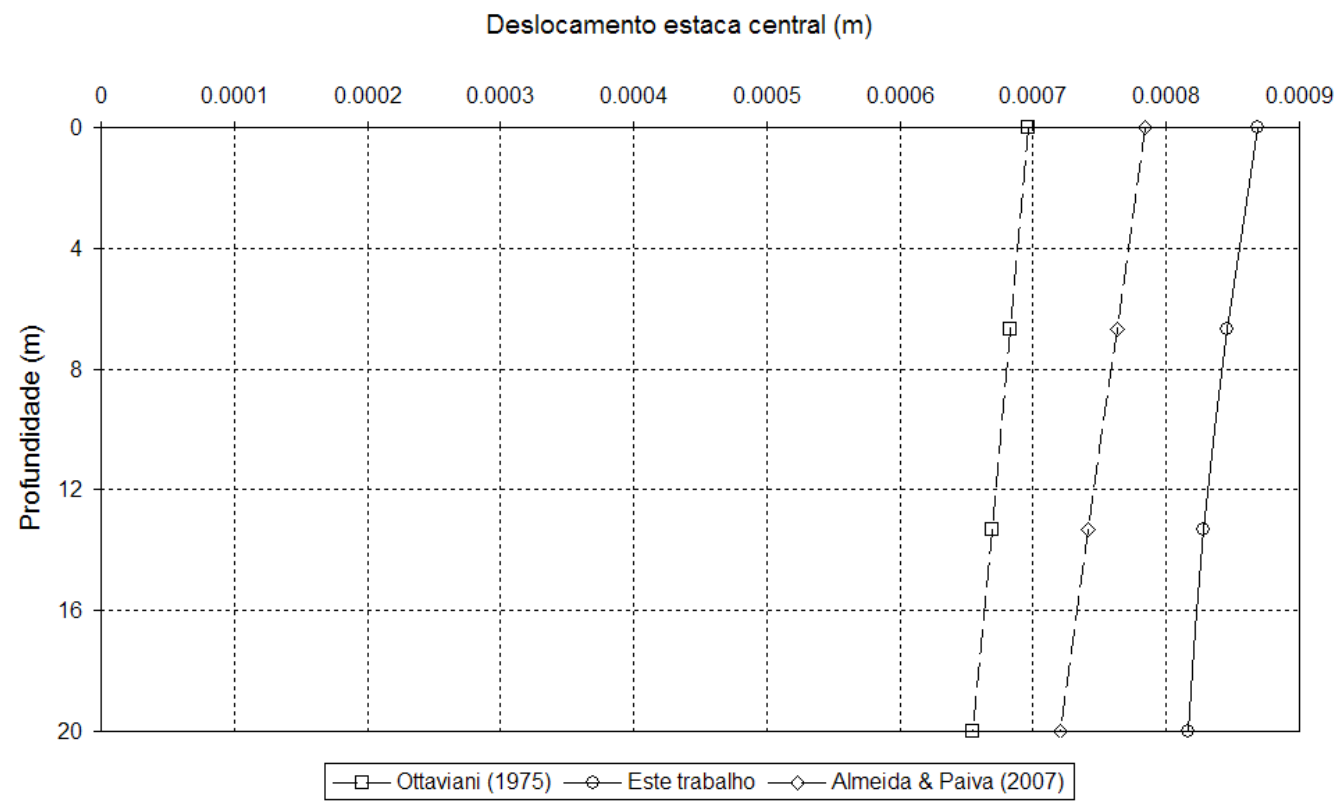

Figura 6.11: Deslocamento vertical da estaca central

de contorno, permite que a malha se extenda até uma distância maior. Desta forma, o resultado obtido por Almeida e Paiva (2007) recebe menor influência dos limites da malha e a solução se aproxima mais da obtida com a formulação deste trabalho, a qual permite simular a camada como um meio de fato infinito nas direções radiais. O resultado de Ottaviani (1975) foi 19,8 \% inferior ao deste trabalho tanto para o topo como para a base da estaca, enquanto que o resultado de Almeida e Paiva (2007) foi 9,7\% inferior para o topo e $11,8 \%$ inferior para a base.

Outro dado interessante de ser avaliado é a influência de uma estaca sobre a outra. Considerando a numeração de 1 a 3 apresentada na figura 6.9, verificou-se que o deslocamento vertical do topo da estaca 3 é 25,5\% superior ao das estacas 1 e 13,8 \% superior ao das estacas 2. Além disso foi observada uma significativa inclinação das estacas 1 e 2 na direção da estaca 3, com o topo se tornando mais próximo do centro da malha e a base se tornando mais distante. Desta forma, verificou-se que a distância horizontal entre o topo e base das estacas 1 resultou igual a 32,1\% do deslocamento vertical de seu topo, enquanto que para as estacas 2 este valor foi de $23,6 \%$. 


\subsection{Interação de placas com o solo}

\subsubsection{Lâmina apoiada em um semi-espaço infinito}

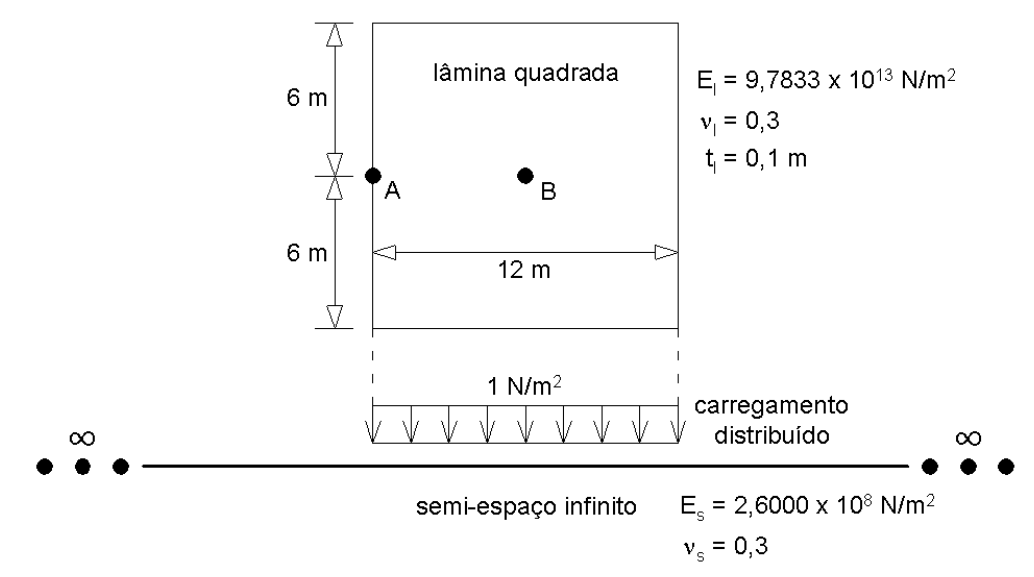

Figura 6.12: Lâmina apoiada no solo

Neste exemplo é analisada uma lâmina apoiada no solo, conforme ilustrado na figura 6.12. O solo é modelado com o MEC como um semi-espaço infinito, homogêneo, isotrópico e elástico-linear, com módulo de elasticidade de $2,6000 \times 10^{8} \mathrm{~N} / \mathrm{m}^{2}$ e coeficiente de Poisson de 0,3. A lâmina, por sua vez, é simulada com o MEF e suas características físicas são módulo de elasticidade de $9,7833 \times 10^{13} \mathrm{~N} / \mathrm{m}^{2}$ e coeficiente de Poisson de 0,3 . A lâmina é quadrada, com $12 \mathrm{~m}$ de aresta e $0,1 \mathrm{~m}$ de espessura, e sobre ela é aplicado um carregamento uniformemente distribuído de $1 \mathrm{~N} / \mathrm{m}^{2}$. O ponto $A$ está posicionado no ponto médio de uma das arestas e o ponto B corresponde ao centro da lâmina.

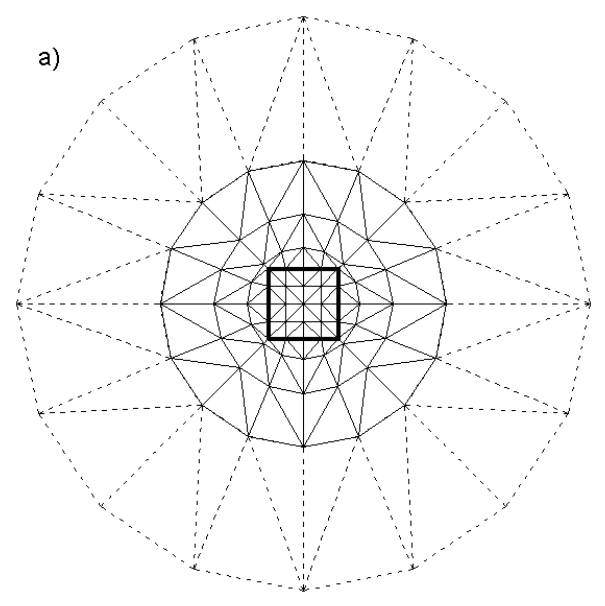

b)

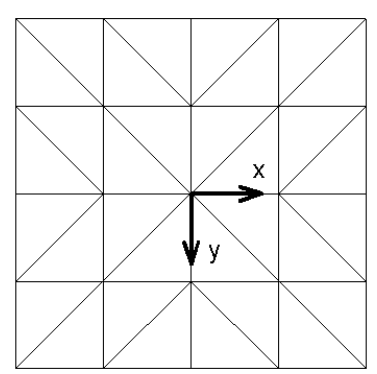

Figura 6.13: Malhas de EF/EC/ECI empregadas

Na figura 6.13 são apresentadas as malhas empregadas na simulação. A malha do 
solo, representada em 6.13a, é composta por 128 ECs e 32 ECIs. As linhas tracejadas representam os ECIs, e o quadrado destacado no centro corresponde ao contato do solo com a lâmina. A malha da lâmina, ilustrada em 6.13b juntamente com o sistema de coordenadas globais, é composta por 32 EFs do tipo DKT/FF.

\begin{tabular}{|c|c|c|c|c|}
\cline { 2 - 5 } \multicolumn{1}{c|}{} & Ponto $A$ & Erro (\%) & Ponto $B$ & Erro (\%) \\
\hline Este trabalho & 3,41 & - & 3,76 & - \\
\hline Almeida (2003b) & 3,54 & 3,8 & 3,79 & 0,8 \\
\hline Messafer e Coates (1989) & 3,00 & 12,0 & 3,63 & 3,5 \\
\hline \hline Paiva e Butterfield (1997) & 2,88 & 15,5 & 3,26 & 13,3 \\
\hline
\end{tabular}

Tabela 6.2: Deslocamento vertical $\left(m \times 10^{-8}\right)$

São apresentados na tabela 6.2 os deslocamentos verticais calculados para os pontos $A$ e $B$, incluindo os resultados obtidos por outros autores. Os erros são calculados tomando como referência os valores obtidos com a formulação desenvolvida neste trabalho. Conforme pode ser observado, houve maior concordância dos valores obtidos neste trabalho com os de Almeida (2003b) quando comparado aos demais autores. Este resultado era esperado pois, exceto pela presença dos ECIs, a formulação de Almeida (2003b) é a mesma deste trabalho.

Outro dado importante de ser analisado é o momento fletor ao longo da lâmina nas direções $x$ e $y$ do sistema de coordenadas globais, conforme ilustrado no figura 6.13. Considerando os pontos $A$ e $B$ foi obtida a tabela 6.3 .

\begin{tabular}{|c|c|c|c|c|c|c|}
\cline { 2 - 7 } \multicolumn{1}{c|}{} & $m x_{B}$ & Erro (\%) & $m y_{B}$ & Erro (\%) & $m y_{A}$ & Erro (\%) \\
\hline Este trabalho & 3,40 & - & 3,40 & - & 2,34 & - \\
\hline Messafer e Coates (1989) & 3,39 & 0,3 & 3,56 & 4,7 & 2,63 & 12,4 \\
\hline Paiva e Butterfield (1997) & 3,14 & 7,6 & 3,14 & 7,6 & 2,36 & 0,9 \\
\hline
\end{tabular}

Tabela 6.3: Momento fletor nos pontos $A$ e $B$

Os momentos são expressos na tabela em $N m / m$, e os erros são calculados tomando como referência os valores obtidos com a formulação desenvolvida neste trabalho. Dada a simetria do problema, espera-se que no ponto central $B$ o valor do momento seja o mesmo nas direções $x$ e $y$. Esta simetria foi obtida na formulação deste trabalho e também no trabalho de Paiva e Butterfield (1997), entretanto houve uma pequena diferença no trabalho de Messafer e Coates (1989). Pode ser observado pelos erros calculados que o resultado deste trabalho tem boa concordância com Messafer e Coates (1989) no ponto $B$, e boa concordância com Paiva e Butterfield (1997) no ponto $A$. 


\subsubsection{Lâmina apoiada em uma camada finita}

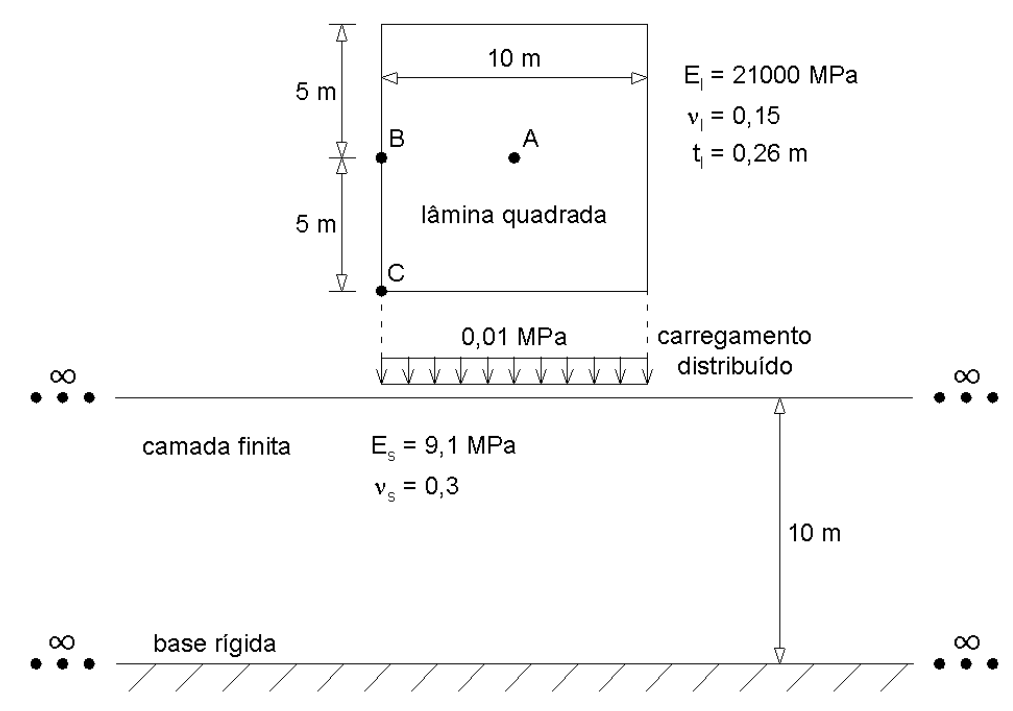

Figura 6.14: Lâmina apoiada em meio finito

Neste exemplo, considera-se uma lâmina quadrada apoiada em uma camada de solo finita, conforme ilustrado na figura 6.14. O solo é representado como um meio tridimensional, homogêneo, isotrópico, elástico e linear. A camada tem $10 \mathrm{~m}$ de espessura, coeficiente de Poisson 0,3, módulo de elasticidade $E_{s}=9,1 \mathrm{MPa}$ e está apoiada em um superfície rígida. A lâmina tem $0,26 \mathrm{~m}$ de espessura, $10 \mathrm{~m}$ de aresta, módulo de elasticidade de $21000 \mathrm{MPa}$ e coeficiente de Poisson 0,15. É aplicada na lâmina um carregamento vertical e uniformemente distribuído de 0,01 MPa. O ponto $A$ está posicionado no centro da lâmina, o ponto $B$ está posicionado no ponto médio de uma das bordas e o ponto $C$ em um dos vértices.

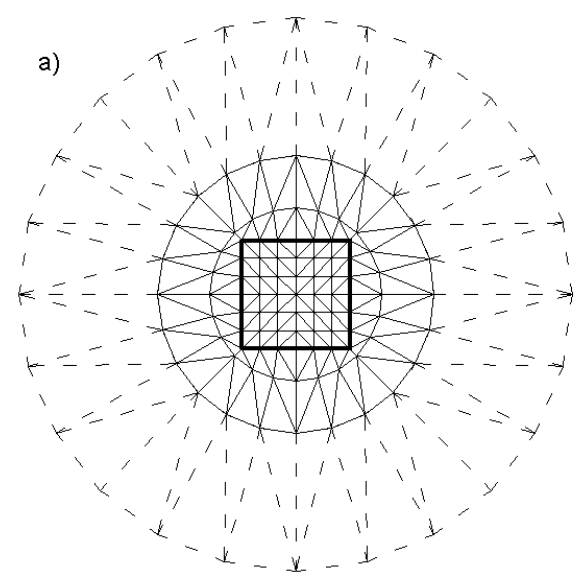

b)

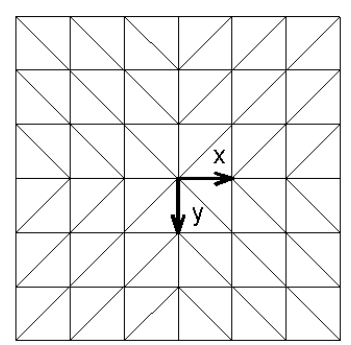

Figura 6.15: Malha de EF/EC/ECI empregada

A figura 6.15 apresenta as malhas de elementos finitos (EF), elementos de contorno 
(EC) e elementos de contorno infinitos (ECI) empregadas na simulação do problema. Em 6.15 é ilustrada uma visão em planta da malha utilizada para modelar a superfície da camada de solo e a base rígida, totalizando 336 ECs e 96 ECIs. O quadrado destacado no centro indica a posição da lâmina na superfície. Em 6.15b pode ser visualizada a malha com 72 EFs empregada para simular a lâmina, juntamente com o sistema de coordenadas global.

Foram calculados os deslocamentos nos pontos $A, B$ e $C$ e o momento fletor no ponto $A$ em torno do eixo $y$ do sistema global. Os resultados obtidos estão organizados na tabela 6.4, juntamente com valores fornecidos por outros autores.

\begin{tabular}{|c|c|c|c|c|}
\cline { 2 - 5 } \multicolumn{1}{c|}{} & $d_{A}(\mathrm{~mm})$ & $d_{B}(\mathrm{~mm})$ & $d_{C}(\mathrm{~mm})$ & $m_{A}(\mathrm{kNm} / \mathrm{m})$ \\
\hline Fraser e Wardle (1976) & 7,30 & 4,50 & 2,80 & 6,20 \\
\hline Kolar e Nemec (1983) & 5,36 & 4,73 & 3,76 & 3,09 \\
\hline \hline Sadecka (2000) & 6,18 & 3,97 & 2,25 & 6,58 \\
\hline Almeida e Paiva (2004b) & 6,47 & 4,62 & 2,95 & 6,22 \\
\hline Este trabalho & 6,50 & 4,56 & 2,72 & 6,59 \\
\hline
\end{tabular}

Tabela 6.4: Resultados

O termo $d_{A}$ se refere ao deslocamento vertical no ponto $A, d_{B}$ ao deslocamento vertical no ponto $B, d_{C}$ ao deslocamento vertical no ponto $C$ e $m_{A}$ se refere ao momento fletor no ponto $A$ em torno do eixo $y$ do sistema de coordenadas global. Conforme pode ser observado, existe boa concordância dos valores obtidos com os dos demais autores. O deslocamentos nos pontos $A, B$ e $C$ foram próximos aos de Almeida e Paiva (2004b), e o momento fletor no ponto $A$ foi próximo ao obtido por Sadecka (2000).

\subsubsection{Lâmina apoiada em solo estratificado}

Neste exemplo, considera-se uma lâmina quadrada apoiada em um solo composto por quatro camadas, conforme ilustrado na figura 6.16. Cada camada de solo é representada como um meio tridimensional, homogêneo, isotrópico, elástico e linear. A primeira camada tem módulo de elasticidade igual a $100 M P a$, a segunda tem um módulo de elasticidade de $80 M P a$, a terceira módulo de $60 M P a$ e a quarta de $100 M P a$, sendo o coeficiente de poisson constante e igual a 0,3 em todo o maciço. As camadas têm $10 \mathrm{~m}$ de espessura, e estão apoiadas em uma base rígida. A lâmina tem 0,5 $\mathrm{m}$ de espessura, $10 \mathrm{~m}$ de aresta, módulo de elasticidade de $15000 \mathrm{MPa}$ e coeficiente de Poisson 0,2. É aplicada na lâmina um carregamento vertical e uniformemente distribuído de 0,1 MPa. O ponto $A$ está 


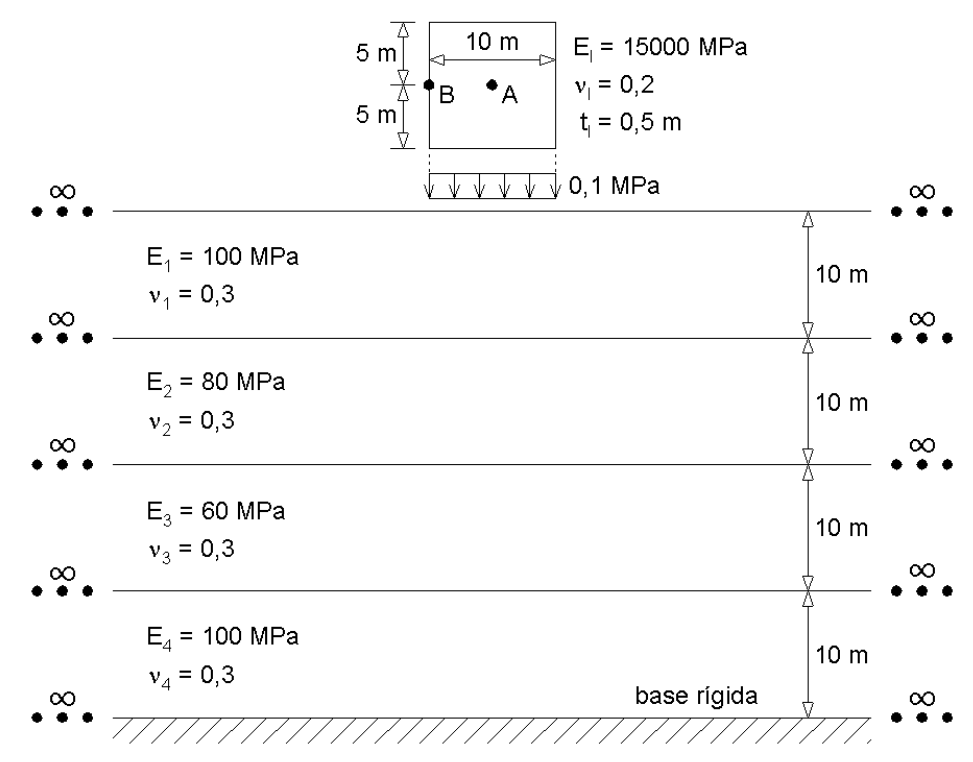

Figura 6.16: Lâmina apoiada em meio não-homogêneo

posicionado no centro da lâmina, e o ponto $B$ está posicionado no ponto médio de uma das bordas.

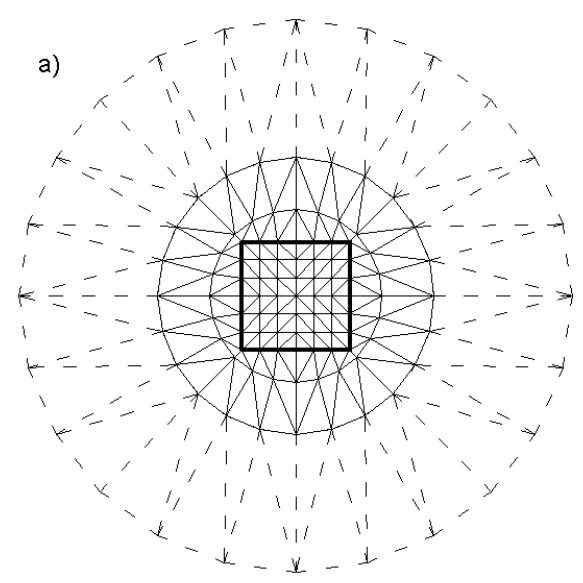

b)

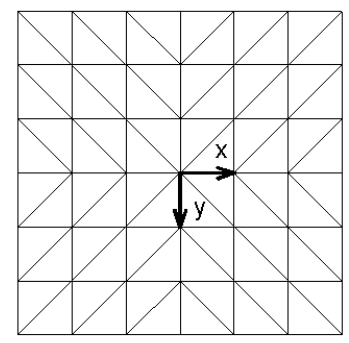

Figura 6.17: Malha de EF/EC/ECI empregada

A figura 6.17 apresenta as malhas de elementos finitos (EF), elementos de contorno (EC) e elementos de contorno infinitos (ECI) empregadas na simulação do problema. Em 6.17a é ilustrada uma visão em planta da malha utilizada para modelar a superfície, os contatos entre camadas e a base rígida, totalizando 840 ECs e 240 ECIs. O quadrado destacado no centro indica a posição da lâmina na superfície. Em 6.17b pode ser visualizada a malha com 72 EFs empregada para simular a lâmina, juntamente com o sistema de coordenadas global.

Foram calculados os deslocamentos nos pontos $A$ e $B$, e os resultados obtidos estão 
organizados na tabela 6.5, juntamente com valores fornecidos por outros autores.

\begin{tabular}{|c|c|c|}
\cline { 2 - 3 } \multicolumn{1}{c|}{} & $d_{A}(\mathrm{~cm})$ & $d_{B}(\mathrm{~cm})$ \\
\hline Este trabalho & 0,97 & 0,74 \\
\hline Wardle e Fraser (1974) & 1,07 & 0,78 \\
\hline Fraser e Wardle (1976) & 1,14 & 0,87 \\
\hline Y. H. Wang e Yue (2003) & 1,20 & 0,89 \\
\hline
\end{tabular}

Tabela 6.5: Deslocamentos verticais

Os termos $d_{A}$ e $d_{B}$ se referem ao deslocamento vertical nos pontos $A$ e $B$, respectivamente. Conforme pode ser observado, houve boa concordância dos valores obtidos com os dos demais autores. Para complementar este exemplo, foi calculado também o momento fletor em torno do eixo global $x$, conforme ilustrado na figura 6.17. O momento fletor no ponto $A$ resultou igual a $3,83 \times 10^{-2} \mathrm{kNm} / \mathrm{m}$, e no ponto $B$ igual a $2,20 \times 10^{-2} \mathrm{kNm} / \mathrm{m}$.

\subsection{Interação de placas estaqueadas com o solo}

\subsubsection{Placa com uma estaca em solo homogêneo}

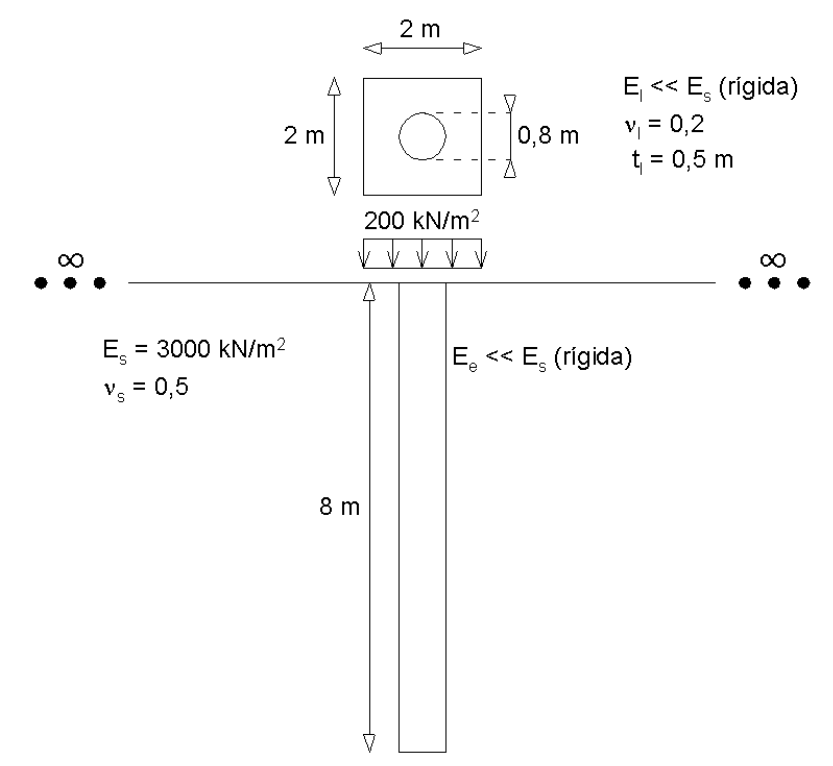

Figura 6.18: Placa com uma estaca apoiada em meio homogêneo

O objetivo deste exemplo é estudar a interação de uma placa com uma estaca com o solo, conforme apresentado na figura 6.18. O solo é representado como um semi-espaço infinito, tridimensional, homogêneo, isotrópico, elástico e linear, com módulo de elasticidade $E_{s}$ igual a $3000 \mathrm{kN} / \mathrm{m}^{2}$ e coeficiente de Poisson 0,5 . A estaca tem $0,8 \mathrm{~m}$ de diâmetro e 8 
$m$ de comprimento, enquanto que e placa é quadrada em planta, com $2 m$ de aresta, 0,5 $m$ de espessura e coeficiente de Poisson de 0,2. Sobre toda a área da placa é aplicado um carregamento vertical e uniformemente distribuído de $200 \mathrm{kN} / \mathrm{m}^{2}$. Tanto a placa como a estaca são admitidas, em princípio, como rígidas, sendo escolhidos para elas valores de módulo de elasticidade da ordem de $10^{6} E_{s}$. Foi verificado que, a partir deste valor, a variação nos deslocamentos ao longo dos nós da placa e estaca é insignificante.

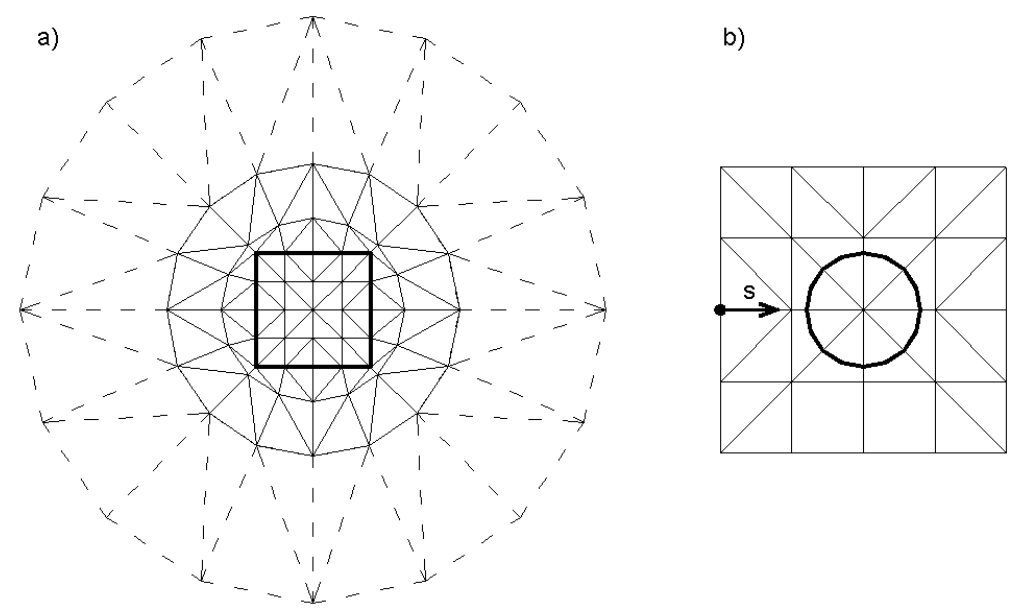

Figura 6.19: Malha de EF/EC/ECI empregada

A malha de elementos de contorno (EC), elementos de contorno infinitos (ECI) e elementos finitos (EF) utilizada para simular este problema é ilustrada na figura 6.19. Em 6.19a é apresentada uma vista em planta da malha empregada para a superfície do solo, sendo composta por 96 ECs e 32 ECIs. O quadrado destacado no centro indica a posição da placa, cuja malha de 32 EFs é apresentada em 6.19b. Nesta figura, o círculo destacado no centro indica a posição da estaca em relação à placa, e o eixo $s$ tem origem no ponto médio da aresta da placa. A estaca é simulada empregando o EF com 14 parâmetros nodais definido na Seção 5.3, sendo seu eixo perpendicular ao plano da malha do solo.

Foi obtido um deslocamento vertical de 4,17 $\mathrm{cm}$ para o topo da estaca. Este valor tem grande concordância com o resultado de 4,13 cm obtido nos trabalhos de Paiva e Trondi (1999) e Butterfield e Banerjee (1971), sendo a diferença inferior a 1 \%. Para estudar a relevância da presença dos ECIs, o mesmo exemplo foi executado empregando para o solo somente a malha de ECs apresentada na figura 6.19 a. Foi obtido então um deslocamento vertical de 3,91 cm para o topo da estaca, resultando em um erro de 5,3\% em relação ao valor de referência. Isto demonstra que o emprego dos ECIs contribui para a convergência do resultado. 
Considerando o eixo $s$ indicado na figura 6.19b o deslocamento ao longo da placa resulta praticamente constante. Foi verificado também que não há alteração nos resultados caso o carregamento distribuído de $200 \mathrm{kN} / \mathrm{m}^{2}$ seja substituído por uma força resultante de $800 k N$ aplicada diretamente no topo da estaca. Este comportamento era esperado devido à alta rigidez da placa e à simetria das cargas aplicadas.

Na segunda parte deste exemplo, para que possa ser analisada a flexão da placa com a estaca, o módulo de elasticidade destes elementos estruturais é reduzido para $3 \times 10^{5}$ $k N / m^{2}$. Como este valor é apenas cem vezes superior ao adotado para o solo, a variação dos deslocamentos ao longo do eixo $s$ indicado na figura $6.19 \mathrm{~b}$ não pode ser ignorada. Além disso, ocorre uma diferença significativa nos resultados quando a carga distribuída é substituída por uma resultante concentrada no topo da estaca. Esta diferença pode ser observada na figura 6.20 .

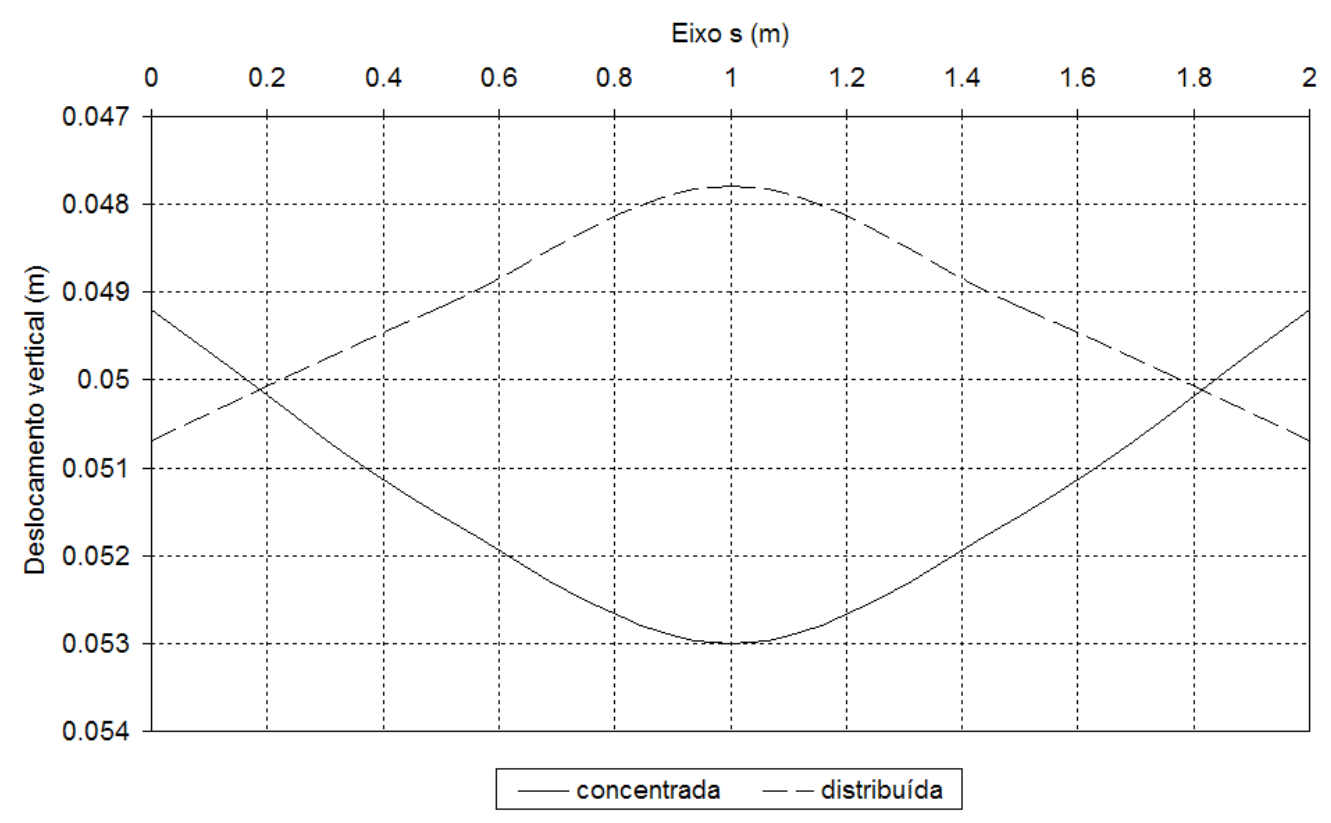

Figura 6.20: Deslocamento ao longo do eixo $s$

Na figura, é apresentado o deslocamento vertical calculado ao longo do eixo $s$ considerando a carga distribuída de $200 \mathrm{kN} / \mathrm{m}^{2}$ e a carga concentrada equivalente de 800 $k N$. Conforme pode ser observado, quando o carregamento distribuído é aplicado a estaca exerce uma punção na placa. Por outro lado, quando a carga é aplicada diretamente na estaca, ela desloca o centro da placa para baixo. Em termos de projeto estas situações são totalmente distintas, sendo necessário, na prática, distinguir de forma clara um caso do outro. 


\subsubsection{Placa com quatro estacas em solo homogêneo}

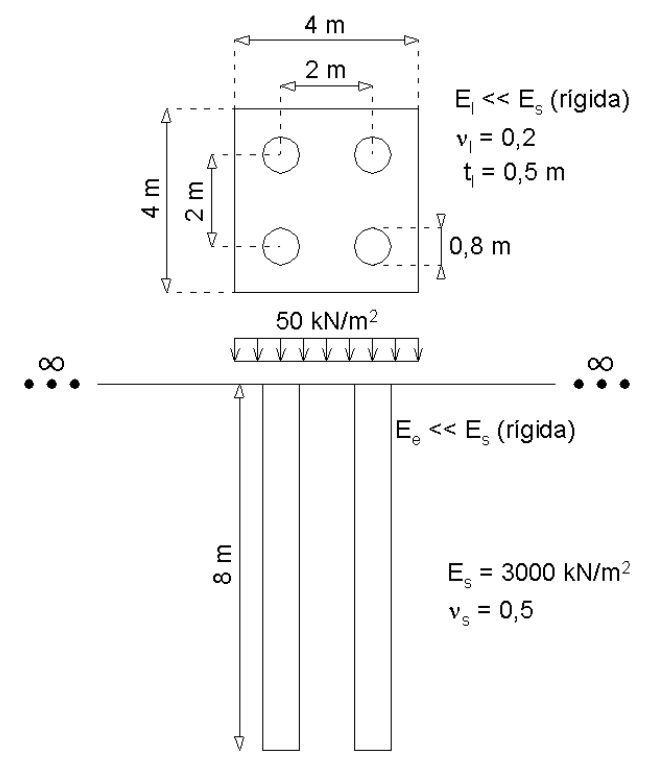

Figura 6.21: Placa quatro estacas apoiada em meio homogêneo

Considera-se uma placa com quatro estacas cilíndricas e verticais imersas no solo, conforme ilustrado na figura 6.21. O solo é representado por um semi-espaço infinito, homogêneo, isotrópico e elástico-linear, com módulo de elasticidade $E_{s}$ de $3000 \mathrm{kN} / \mathrm{m}^{2}$ e coeficiente de Poisson de 0,5 . As estacas são rígidas, com $0,8 m$ de diâmetro, $8 m$ de comprimento e estão espaçadas de $2 \mathrm{~m}$, enquanto que a placa, que também é rígida, é quadrada, com $4 m$ de aresta, 0, $5 m$ de espessura e coeficiente de Poisson 0,2. Para que os elementos estruturais possam ser considerados rígidos, adotou-se para eles um módulo de elasticidade da ordem de $10^{6} E_{s}$. Sobre a placa é aplicado um carregamento uniformemente distribuído de $50 \mathrm{kN} / \mathrm{m}^{2}$.

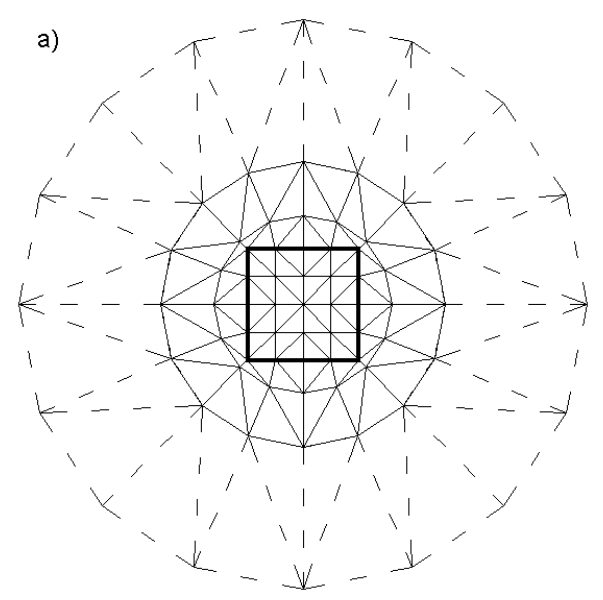

b)

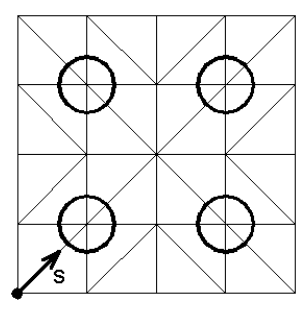

Figura 6.22: Malha de EF/EC/ECI empregada 
A malha de elementos de contorno (EC), elementos de contorno infinitos (ECI) e elementos finitos $(\mathrm{EF})$ utilizada para simular este problema é ilustrada na figura 6.22. Em 6.22a é apresentada uma vista em planta da malha empregada para a superfície do solo, sendo composta por 96 ECs e 32 ECIs. O quadrado destacado no centro indica a posição da placa, cuja malha de 32 EFs é apresentada em 6.22 b. Nesta figura, os círculos destacados indicam a posição das estacas em relação à placa, e o eixo $s$ tem origem em um dos vértices. As estacas são simuladas empregando o EF com 14 parâmetros nodais definido na Seção 5.3 , sendo seus eixos perpendiculares ao plano da malha do solo.

Foi calculado um deslocamento vertical de $2,59 \mathrm{~cm}$ o qual, dada a rigidez da placa, é praticamente constante ao longo de toda a sua área. Este valor é bem próximo do obtido no trabalho de Paiva e Trondi (1999), que foi de 2,62 cm, com uma diferença de apenas 1,1 \%. O valor fornecido por Butterfield e Banerjee (1971), que é de 2,79 cm, também pode ser considerado próximo, com uma diferença de 7,2 \%. Para estudar a relevância da presença dos ECIs, o mesmo exemplo foi executado empregando para o solo somente a malha de ECs apresentada na figura 6.22 a. Foi obtido então um deslocamento vertical de 2,41 cm para toda a placa, resultando em um erro de 8,0\% em relação ao valor obtido por Paiva e Trondi (1999) e em um erro de 13,6 \% em relação ao valor obtido por Butterfield e Banerjee (1971). Isto demonstra que, também neste exemplo, o emprego dos ECIs contribui para a convergência do resultado.

Na sequência, para permitir a análise da flexão da placa com estacas, o módulo de elasticidade destes elementos estruturais é reduzido para $3 \times 10^{5} \mathrm{kN} / \mathrm{m}^{2}$. Sendo este valor apenas cem vezes superior ao adotado para o solo, a variação dos deslocamentos ao longo do eixo $s$ indicado na figura 6.19b não pode ser ignorada. Além disso, ocorre uma diferença significativa nos resultados quando a carga distribuída é substituída por quatro forças concentradas iguais e aplicadas no topo das estacas, de forma que a força resultante seja a mesma do carregamento distribuído. Esta diferença pode ser observada na figura 6.23 ,

Na figura, é apresentado o deslocamento vertical calculado ao longo do eixo $s$ considerando a carga distribuída de $50 \mathrm{kN} / \mathrm{m}^{2}$ e as cargas concentradas equivalentes de 200 $k N$ cada. As linhas escuras verticais indicam a posição do eixo das estacas que o eixo $s$ intercepta. Conforme pode ser observado, quando o carregamento distribuído é aplicado as estacas causam uma punção na placa. Por outro lado, quando a cargas concentradas 


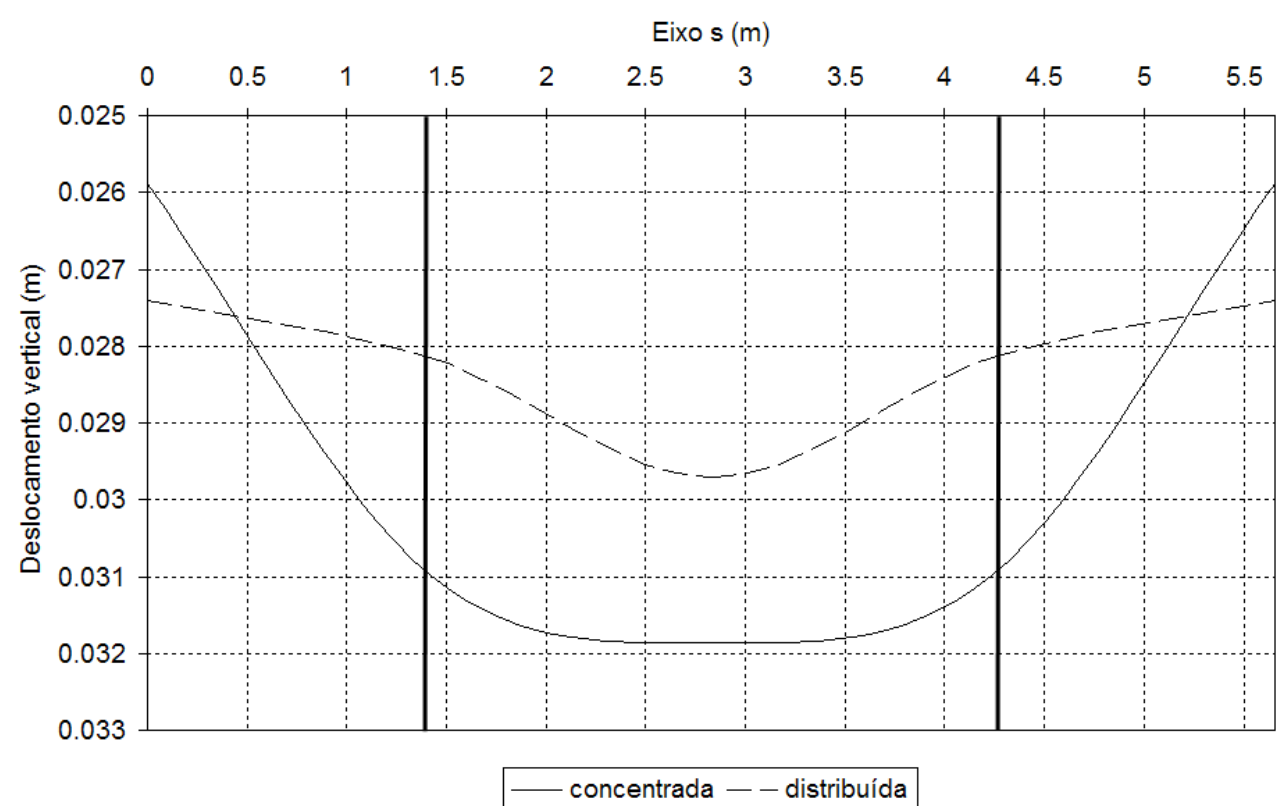

Figura 6.23: Deslocamento ao longo do eixo $s$

são aplicadas diretamente nas estacas, o centro da placa é deslocado para baixo. Assim como no exemplo anterior, em termos de projeto estas situações são totalmente distintas, sendo necessário, na prática, distinguir de forma clara um caso do outro.

\subsubsection{Placa com nove estacas em solo não-homogêneo}

Considera-se uma placa quadrada e com nove estacas interagindo com um solo nãohomogêneo, tal como ilustrado na figura 6.24. Cada camada de solo é representada como um domínio infinito nas direções radiais, homogêneo, isotrópico e elástico-linear, sendo o coeficiente de Poisson constante e igual a zero em todo o maciço. A primeira camada, que contém as estacas, possui módulo de elasticidade de $60 \mathrm{MPa}$ e $15 \mathrm{~m}$ de espessura. A segunda camada, por sua vez, possui módulo de elasticidade igual a $80 M P a$ e uma espessura de $20 \mathrm{~m}$. A camada mais profunda é um semi-espaço infinito com um módulo de elasticidade elevado, igual a $90000 \mathrm{MPa}$. As estacas são todas cilíndricas e iguais, com 0,5 $\mathrm{m}$ de diâmetro, $10 \mathrm{~m}$ de comprimento e módulo de elasticidade igual a 15000 $M P a$, estando espaçadas de $5 \mathrm{~m}$. Como o problema é simétrico, espera-se resultados equivalentes em algumas estacas. Desta forma, na figura 6.24 as estacas são numeradas de 1 a 3 segundo esta equivalência. A placa possui um módulo de elasticidade de 15000 $M P a$, coeficiente de Poisson igual a 0,2, $20 m$ de aresta e 0,5 $m$ de espessura. Sobre toda a placa é aplicado um carregamento distribuído e uniforme de 0,04 MPa. 

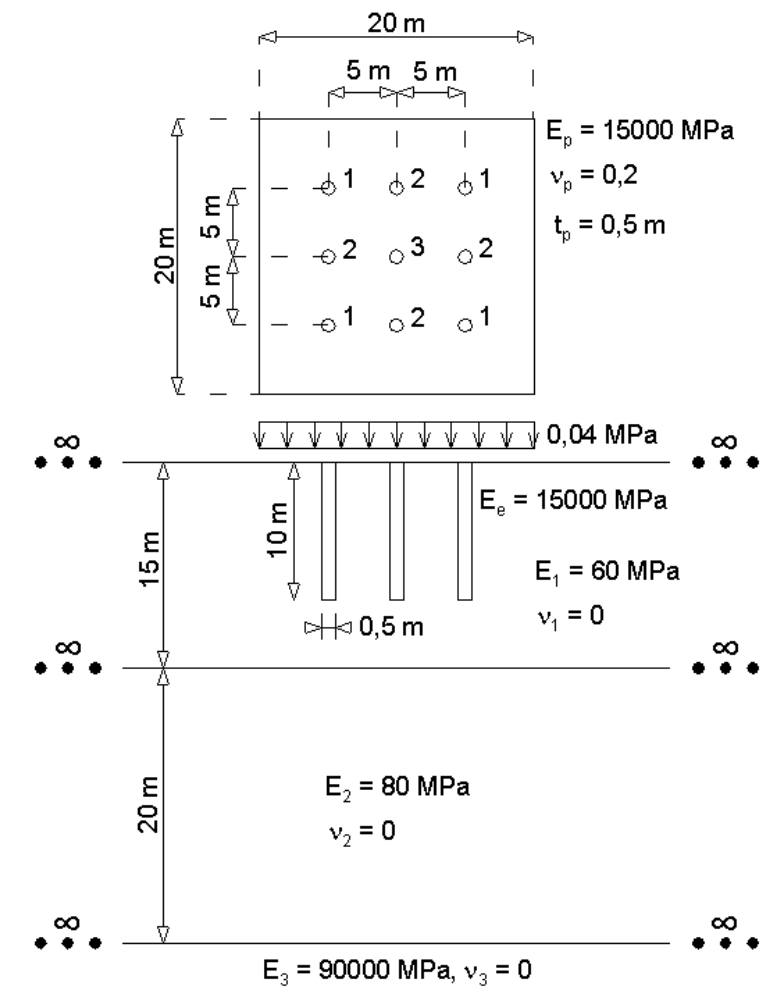

Figura 6.24: Placa estaqueada apoiada em meio não-homogêneo

A figura 6.25 apresenta as malhas de elementos finitos (EF), elementos de contorno (EC) e elementos de contorno infinitos (ECI) empregadas na simulação do problema. Em 6.25 é ilustrada uma visão em planta da malha utilizada para modelar a superfície e os contatos entre camadas, totalizando 480 ECs e 96 ECIs. O quadrado destacado no centro indica a posição da placa na superfície. Em 6.25b pode ser visualizada a malha com 32 EFs empregada para simular a lâmina, juntamente com o sistema de coordenadas global. Os círculos destacados na malha da placa indicam a posição das estacas. As estacas são simuladas com o método dos elementos finitos (MEF), empregando o elemento com 14 parâmetros nodais definido na Seção 5.3. O eixo das estacas é perpendicular ao plano da malha do solo.

A simulação forneceu resultados simétricos conforme esperado, e na figura 6.26 são apresentados os deslocamentos verticais em $m m$ ao longo do eixo das estacas de 1 a 3 . Para a estaca 1 foram obtidos os menores deslocamentos, sendo o de topo igual a 6,8 $\mathrm{mm}$. Para a estaca 2 o deslocamento obtido para o topo foi de 7,2 $\mathrm{mm}$, sendo este valor $5,9 \%$ superior ao da estaca 1 . A estaca central 3, por sua vez, foi a mais deslocada, com um deslocamento de topo igual a 8,4 $\mathrm{mm}$. Este valor é 23,5\% superior ao obtido para o topo da estaca 1. 


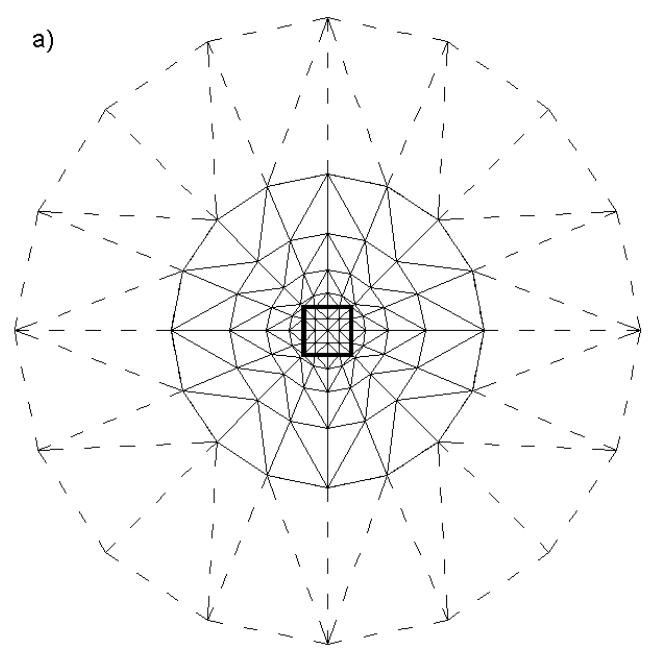

b)

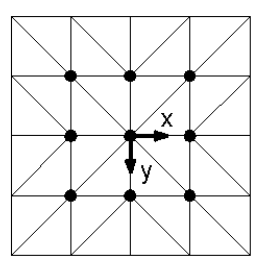

Figura 6.25: Malha de EF/EC/ECI empregada

Para complementar este exemplo, considera-se novamente o mesmo sistema apresentado na figura 6.24 e com as mesmas malhas apresentadas na figura 6.25. Entretanto, neste caso a carga distribuída é substituída por quatro forças concentradas de $4000 k N$, aplicadas no nó de topo das estacas numeradas como 1 na figura 6.24. A força resultante da soma destas cargas é equivalente à do carregamento distribuído previamente considerado, e os novos deslocamentos obtidos da simulação são apresentados na figura 6.27.

Novamente, a simulação forneceu resultados simétricos segundo a numeração da figura 6.24. Comparando as figuras 6.26 e 6.27, observa-se que ocorre um maior deslocamento das estacas do tipo 1 quando as cargas são aplicadas diretamente sobre elas. Este resultado pode ser considerado coerente, pois espera-se que uma maior porcentagem da carga seja transferida para estas estacas neste segundo caso. O deslocamento vertical obtido para o topo da estaca 1 foi de 12,4 $\mathrm{mm}$, representando um aumento muito significativo em relação a valor obtido com o carregamento distribuído. O deslocamento de topo obtido para as demais estacas foi próximo, sendo igual a 7,4 $\mathrm{mm}$ para a estaca 2 e 7,0 $\mathrm{mm}$ para a estaca 3 .

\subsection{Edifício apoiado no solo}

O objetivo deste exemplo é apresentar uma aplicação que demonstre a abrangência da ferramenta numérica desenvolvida neste trabalho, permitindo a análise de diversos tipos de sistemas estruturais diferentes interagindo entre si. O problema a ser estudado, 


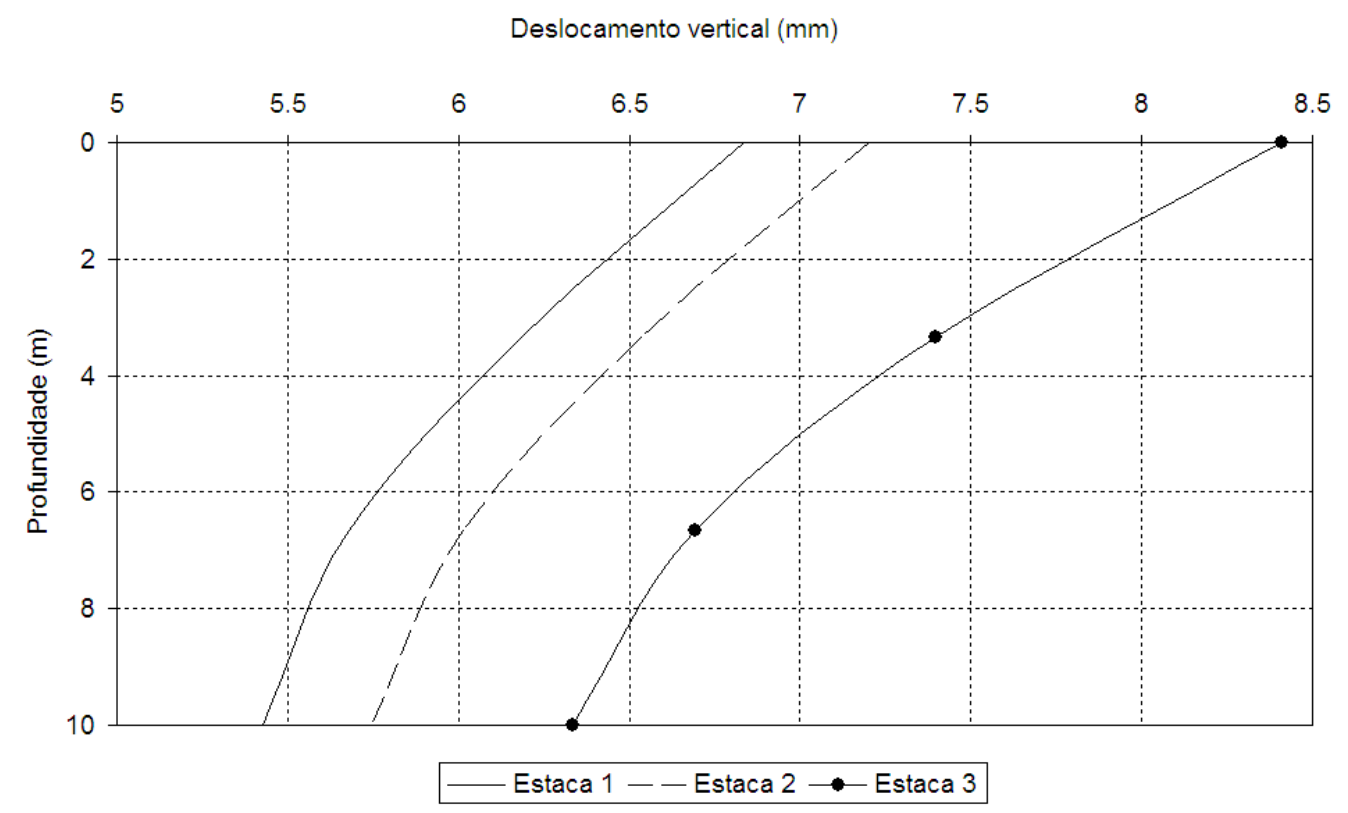

Figura 6.26: Deslocamento vertical das estacas

conforme apresentado na figura 6.28, é o de um edíficio com suas estruturas de fundação e apoiado em um maciço de solos não-homogêneo. Em 6.28 a é ilustrada uma vista em perfil de todo o conjunto, em $6.28 \mathrm{~b}$ é apresentado o pavimento tipo considerado no edifício e em 6.28c é demonstrada uma vista em planta das estruturas de fundação consideradas.

O sistema formado pelas estruturas de fundação do edifício em conjunto com o maciço de solos corresponde exatamente ao exemplo analisado na Seção 6.4.3. Desta forma, é apresentada aqui uma descrição mais resumida deste sistema. O coeficiente de Poisson é constante e igual a zero em todo o maciço, sendo o módulo de elasticidade das camadas igual a 60, 80 e $90000 M P a$, e suas espessuras de $15 m, 20 m$ e $\infty$. As estacas possuem 0,5 $m$ de diâmetro, $10 m$ de comprimento e estão espaçadas de $5 \mathrm{~m}$, enquanto que o radier possui $20 \mathrm{~m}$ de aresta e $0,5 \mathrm{~m}$ de espessura. Todos os materiais modelados com o método dos elementos finitos (MEF) possuem módulo de elasticidade igual a $15000 \mathrm{MPa}$ e coeficiente de Poisson 0,2. Isto inclui todas as lajes, vigas, pilares, estacas e o radier.

O edifício é composto por quatro pavimentos, conforme ilustrado na figura 6.28 a. Todos os pavimentos são iguais ao apresentado na figura $6.28 \mathrm{~b}$, sendo compostos por uma laje de 0,3 $\mathrm{m}$ de espessura, quatro vigas que sustentam a laje e quatro pilares que sustentam as vigas. Todas as vigas e pilares considerados possuem a mesma seção transversal, a qual é quadrada e com $1 m$ de aresta. No radier, a base dos pilares é conectada no topo das estacas de canto, as quais estão numeradas como 1, 3, 7 e 9 na 


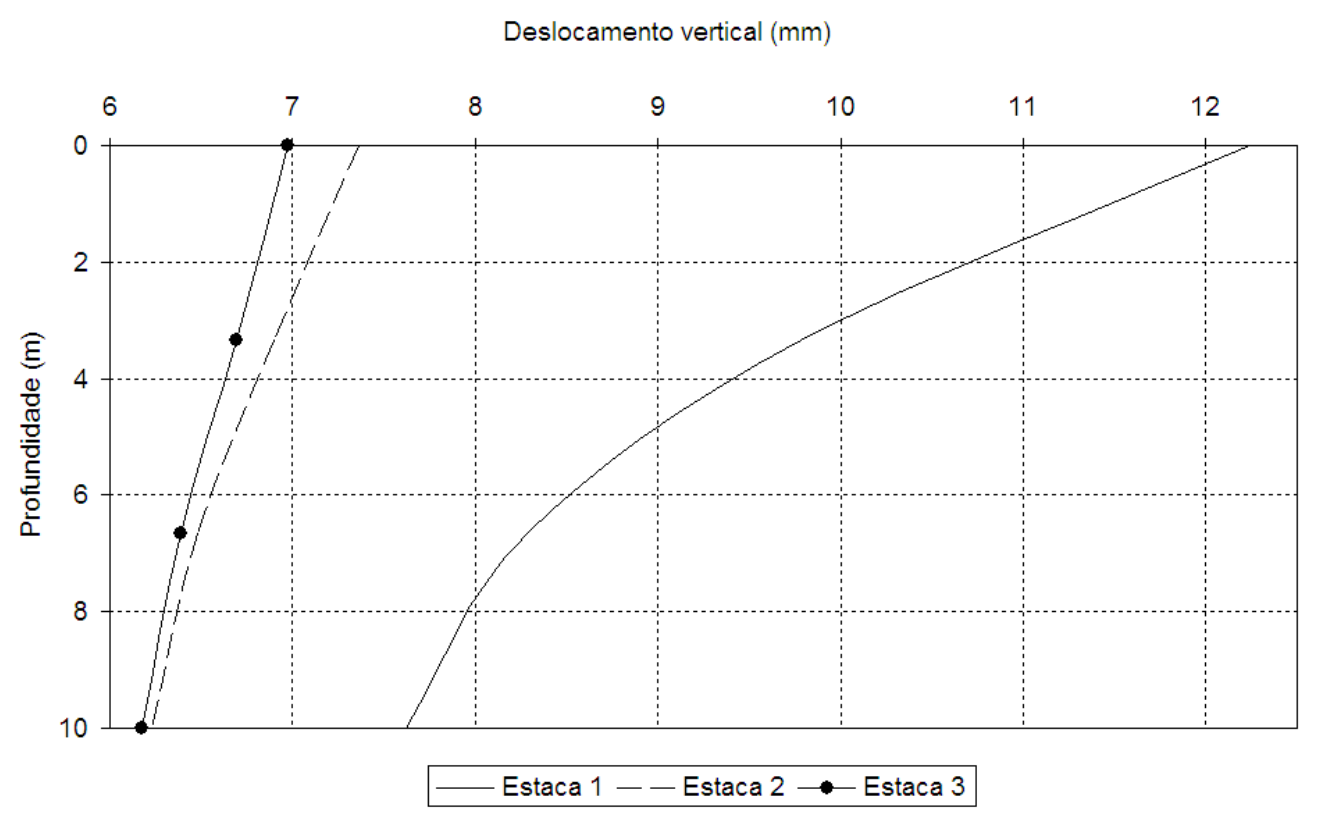

Figura 6.27: Deslocamento vertical para cargas concentradas

figura 6.28 .

As cargas externas consideradas são carregamentos de 0,04 MPa distribuídos sobre as quatro lajes, conforme ilustrado na figura 6.29. É importante comentar que, somando as cargas das quatro lajes, a força resultante total é igual àquela considerada no exemplo 6.4 .3 .

A figura 6.30 apresenta as malhas de elementos finitos (EF), elementos de contorno (EC) e elementos de contorno infinitos (ECI) empregadas na simulação do problema. Em 6.30a é ilustrada uma visão em planta da malha utilizada para modelar a superfície e os contatos entre camadas, totalizando 480 ECs e 96 ECIs. O quadrado destacado no centro indica a posição da placa na superfície.

Em 6.30b pode ser visualizada a malha com 32 EFs empregada para simular o radier, juntamente com a posição das estacas. Por fim, em 6.30 pode ser visualizada a malha de 32 EFs empregada em cada laje. As linhas destacadas nas bordas da laje indicam os EFs de barra utilizados para simular as vigas, somando 16 por pavimento. Além disto, cada trecho de pilar entre pavimentos é dividido em 4 elementos de barra, totalizando 16 EFs por pavimento. Somando todos os pavimentos e o radier, foram empregados no total 160 EFs triangulares e 128 EFs de barra.

As estacas são simuladas com o método dos elementos finitos (MEF), empregando o elemento com 14 parâmetros nodais definido na Seção 5.3. O eixo das estacas é perpen- 

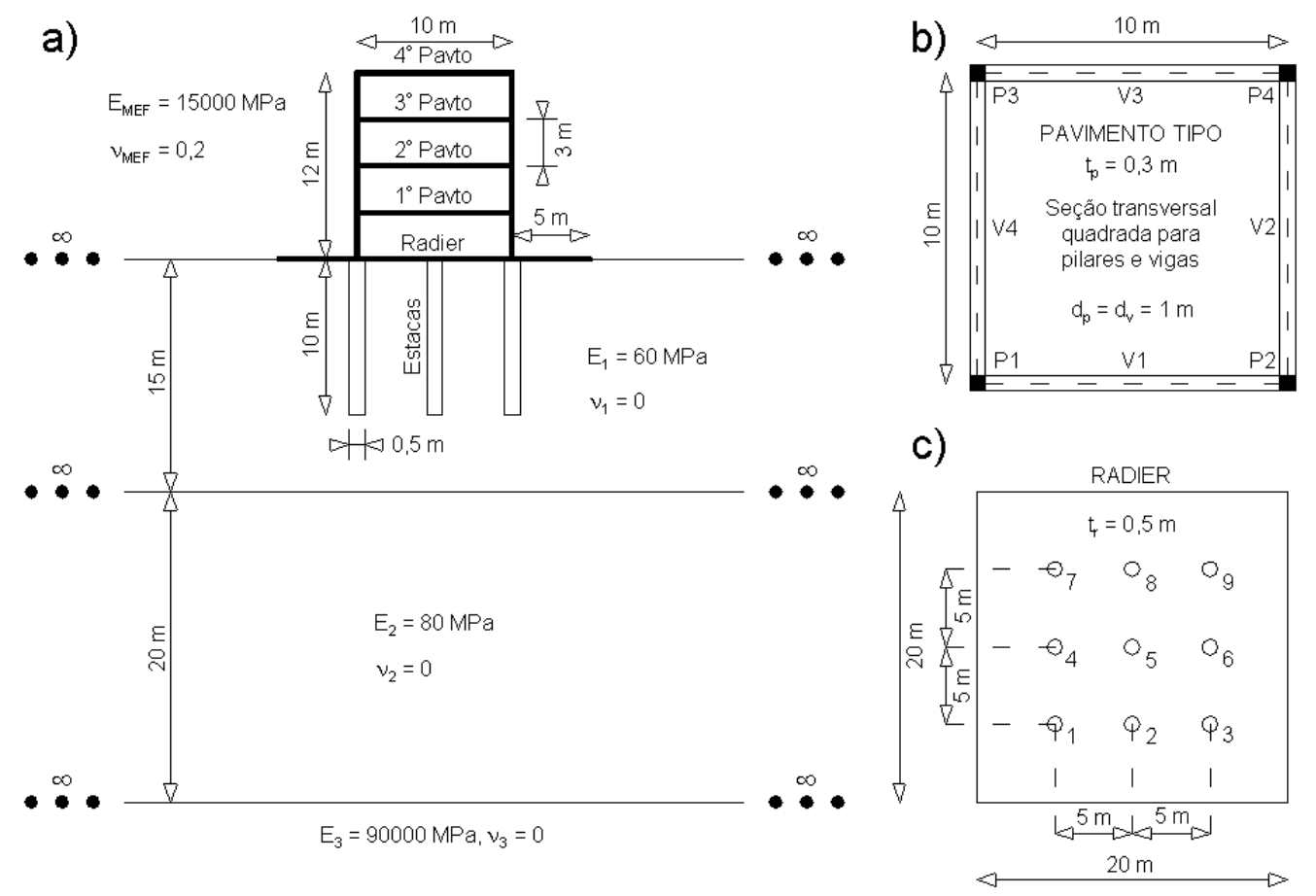

Figura 6.28: Interação edifício-radier-estaca-solo

dicular ao plano da malha do solo.

O primeiro resultado, ilustrado na figura 6.31, é o deslocamento vertical do eixo das estacas. São apresentadas apenas as estacas numeradas como 1, 2 e 5 na figura 6.28k, pois o resultado foi simétrico para as demais. Os pilares de canto foram os que apresentaram maior deslocamento, sendo o valor no topo igual a 12,2 $\mathrm{mm}$. Este resultado pode ser considerado coerente, pois a base dos pilares está posicionada exatamente sobre estas estacas. O deslocamento de topo obtido para as demais estacas foi de 7,3 $\mathrm{mm}$ para a estaca 2 e 6,9 $\mathrm{mm}$ para a estaca 5. Comparando as figuras 6.27 e 6.31, nota-se que o deslocamento de topo das estacas com o edifício é, neste caso, quase equivalente ao caso em que as cargas são diretamente aplicadas nas estacas.

São apresentados na figura 6.32 resultados calculados para o pavimento número 4 , conforme a numeração apresentada na figura 6.28 a. Considera-se os valores ao longo de uma linha diagonal ao pavimento, com extremos nos pilares $P 1$ e $P 4$ conforme indicado na figura $6.28 \mathrm{~b}$. Além do resultado calculado considerando a base flexível ilustrada na figura 6.28 a são colocados também os valores obtidos quando é considerada uma base rígida para o edifício, ou seja, aplicando restrições de deslocamento na base dos pilares. É possível observar que os deslocamentos da laje são significativamente superiores quando a base flexível é considerada, o que pode ser considerado um resultado previsível. O 


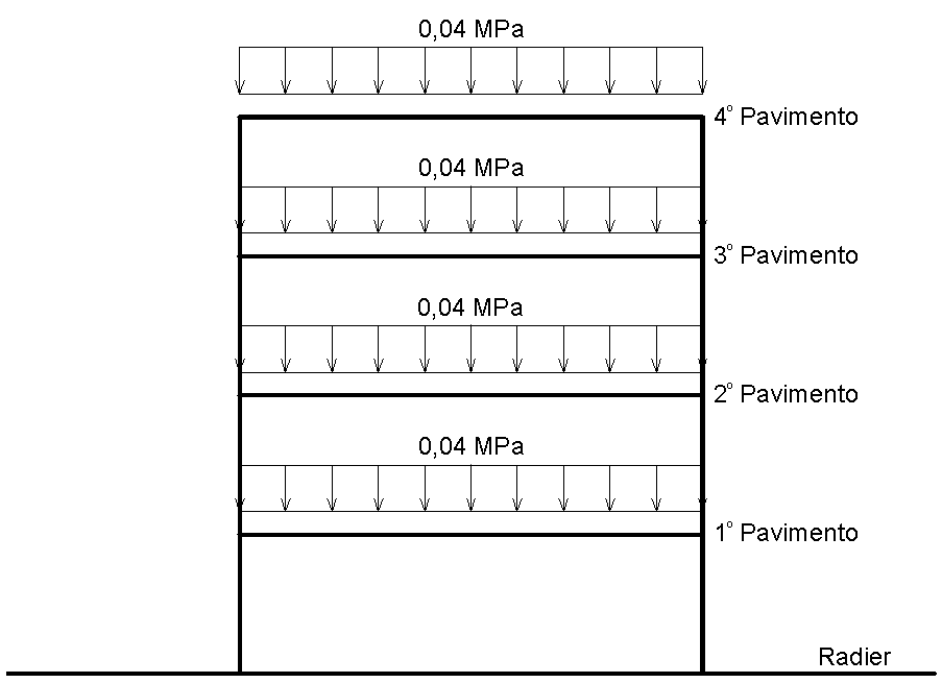

Figura 6.29: Cargas verticais aplicadas

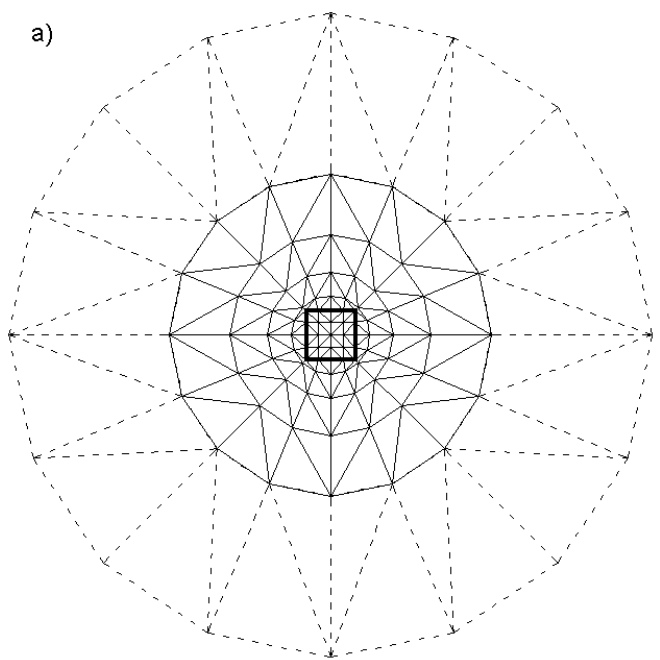

b)

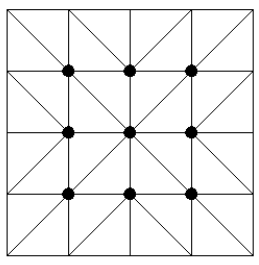

c)

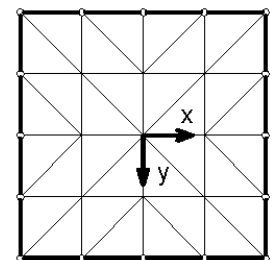

Figura 6.30: Malha de EF/EC/ECI empregada

deslocamento máximo da laje para o caso rígido é de $28,8 \mathrm{~mm}$, enquanto que para o caso flexível este valor atinge $41,0 \mathrm{~mm}$.

Na segunda parte deste exemplo, os carregamentos distribuídos sobre as lajes são substituídos por duas forças concentradas horizontais aplicadas na lateral da edificação, conforme apresentado na figura 6.33. Em 6.33a é ilustrada a vista lateral da estrutura, com as forças aplicadas no plano do pavimento 4. A posição em planta na qual as forças são aplicadas pode ser visualizada na figura $6.33 \mathrm{~b}$.

Para este segundo carregamento, apresenta-se na figura 6.34 os deslocamentos horizontais calculados ao longo do pilar $P 1$, cuja posição pode ser observada na figura $6.28 \mathrm{~b}$. Também para este caso foram feitas duas simulações, uma considerando a base flexível ilustrada na figura 6.28 a e outra considerando uma base rígida, ou seja, aplicando restri- 


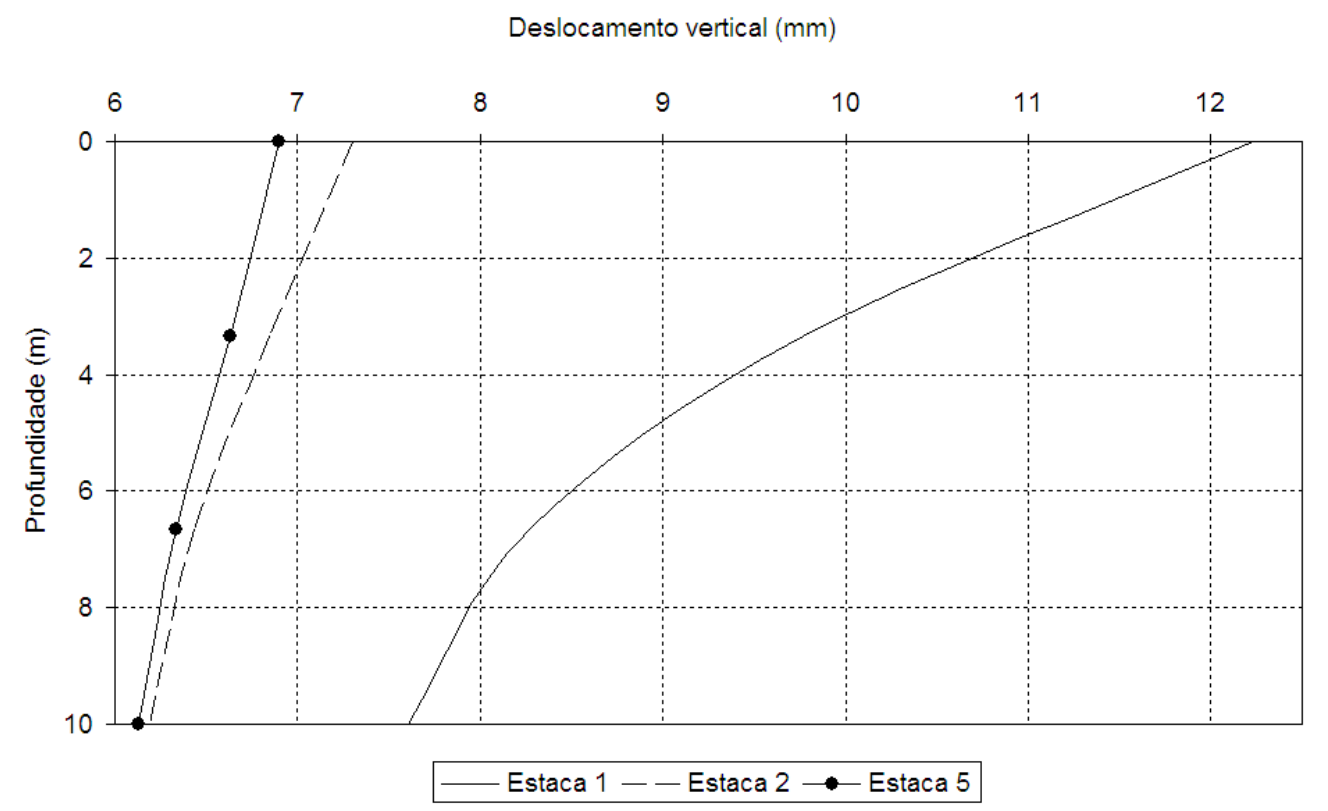

Figura 6.31: Deslocamento vertical para cargas nas lajes

ções de deslocamento na base dos pilares.

Conforme esperado, o pilar se desloca mais quando a base flexível é considerada. Para a base rígida o deslocamento calculado para o topo do pilar foi de $8,9 \mathrm{~mm}$, enquanto que para o caso flexível este valor atinge $11,3 \mathrm{~mm}$. Além disto, analisando os deslocamentos ao longo de todo o pilar nos dois casos, conclue-se que no caso flexível ocorre uma maior inclinação da estrutura. Como os deslocamentos horizontais se tornam excentricidades para as cargas aplicadas na direção vertical, estas diferenças introduzem esforços relevantes que podem agir contra a segurança da estrutura. Isto demonstra a importância de considerar-se a flexibilidade do solo no cálculo de edificações. 


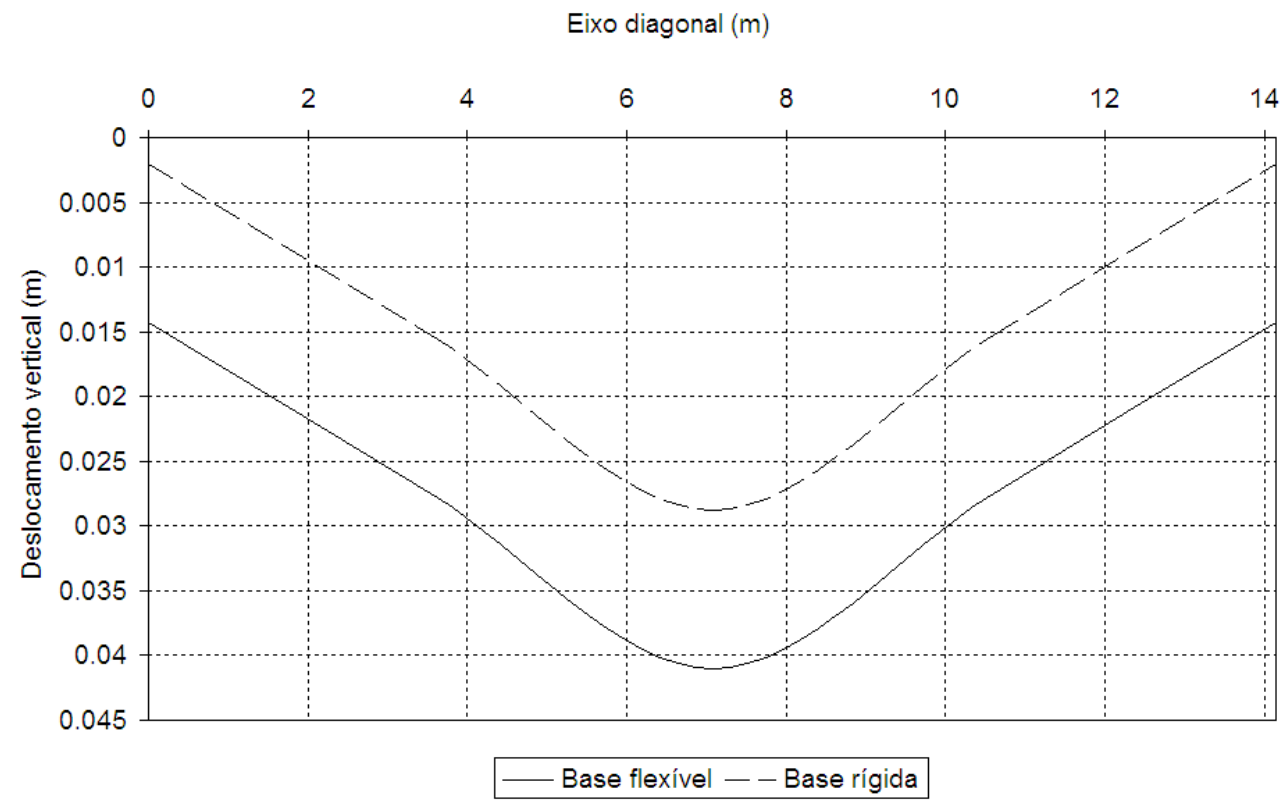

Figura 6.32: Deslocamento vertical no quarto pavimento

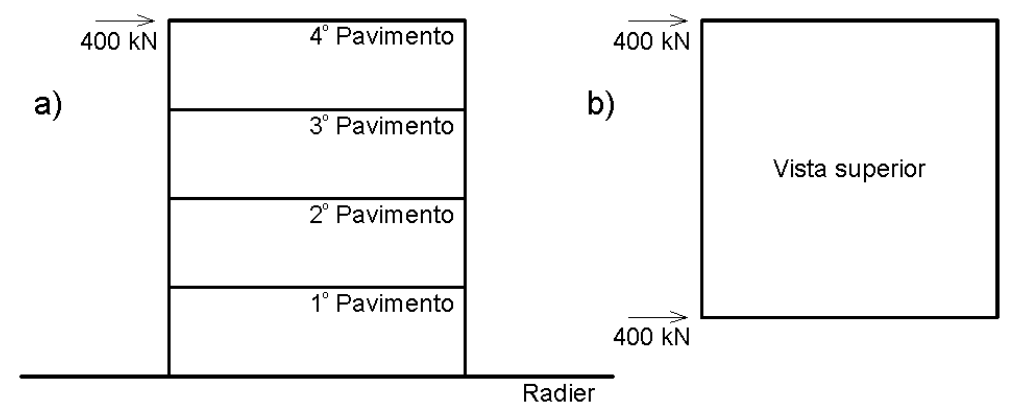

Figura 6.33: Cargas horizontais aplicadas

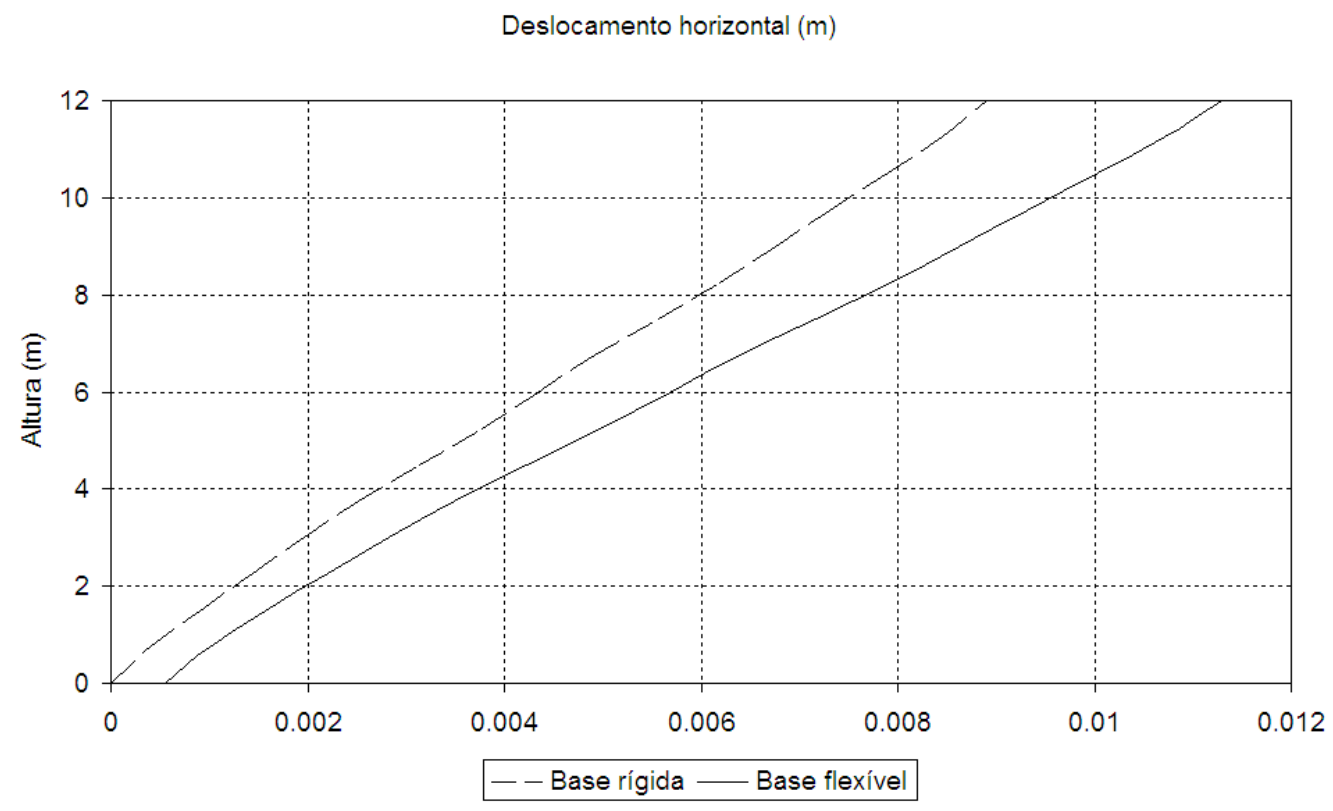

Figura 6.34: Deslocamento horizontal no pilar $P 1$ 


\section{Capítulo 7}

\section{Conclusões}

\subsection{Observações finais}

Neste trabalho de doutoramento foi desenvolvida uma ferramenta numérica para a simulação estática de problemas tridimensionais de interação solo-estrutura. O solo é simulado como um maciço isotrópico, elástico e linear, que pode ser não-homogêneo e estar apoiado em uma superfície de deslocamentos nulos. O programa desenvolvido permite analisar a interação de diversos tipos de estruturas com este maciço, que podem variar desde uma estaca isolada até um edifício com suas estruturas de fundação.

O maciço de solos é modelado com o método dos elementos de contorno (MEC) empregando as soluções fundamentais de Kelvin, válidas para sólidos tridimensionais. Os diferentes domínios em contato no maciço são agrupados utilizando uma técnica alternativa de subregiões, a qual é apresentada em detalhes no Capítulo 3 . Esta formulação é uma das contribuições originais deste trabalho, sendo apresentada em Ribeiro e Paiva (2009). Esta técnica se baseia no relacionamento das soluções fundamentais dos diferentes domínios, permitindo que todo o maciço seja equacionado como um único sólido. Esta estratégia dispensa o emprego de condições de equilíbrio de forças e compatibilidade de deslocamentos nas superfícies de contato entre os diferentes materiais, tornando a formulação mais precisa.

Uma desvantagem desta técnica é que todos os materiais modelados devem ter mesmo coeficiente de Poisson. No entanto esta limitação tem pouca relevância nas aplicações abordadas, pois como neste trabalho se pretende simular o maciço de solos de forma semelhante à formulação de Gibson (1967), em que o módulo de elasticidade é variável 
mas o coeficiente de Poisson é constante. No capítulo 3 foram apresentados dois exemplos nos quais é demonstrada a superioridade desta técnica alternativa sobre a forma clássica de considerar-se sólidos não-homogêneos com o MEC. Além disto, foi concluído que o erro introduzido nos resultados pela limitação do coeficiente de Poisson é pequeno quando comparado à imprecisão da formulação clássica. Desta forma, mesmo em problemas nos quais existem diferentes coeficientes de Poisson a técnica alternativa pode ser mais vantajosa que a clássica.

Com o intuito de reduzir o custo computacional sem prejudicar a precisão dos resultados, neste trabalho são utilizados elementos de contorno infinitos (ECI) nas bordas da malha de EC do maciço de solos para modelar o comportamento das variáveis de campo a grandes distâncias. A formulação do ECI empregado é detalhada no Capítulo 4 e é mais uma contribuição original deste trabalho. Uma das principais vantagens deste ECI é que nenhum grau de liberdade é adicionado à malha original de ECs pela sua presença, tornando seu uso viável em todos os exemplos desta Tese que envolvem domínios infinitos. No Capítulo 4 são apresentados dois exemplos nos quais é demonstrada a eficiência da formulação de ECIs desenvolvida, e concui-se que sua utilização promove uma significativa redução de malha sem prejuízo da precisão dos resultados.

Todas as estruturas que interagem com o maciço de solos são modeladas com o método dos elementos finitos (MEF), incluindo elementos unidimensionais especiais para estacas, elementos de barra para vigas e pilares elementos de casca para as lajes e o radier.

As estacas são modeladas empregando o EF com quatorze parâmetros nodais apresentado em Filho et al. (2005), sendo empregadas funções de forma do quarto grau para aproximar os deslocamentos horizontais, do terceiro grau para as forças horizontais e deslocamentos verticais e do segundo grau para as forças cisalhantes verticais. A presença destas estacas é considerada na formulação do MEC como uma linha de carga, assim como feito em Filho et al. (2005). Entretanto este autor emprega as soluções fundamentais de Mindlin ao modelar o solo, portanto a formulação desenvolvida neste trabalho empregando as soluções fundamentais de Kelvin pode ser considerada original. Os elementos de barra utilizados no edifício possuem dois nós e seis graus de liberdade por nó. Os elementos de casca empregados são triangulares, com três nós e seis graus de liberdade por nó. O acoplamento do MEC com o MEF é feito utilizando uma estratégia semelhante à empregada no trabalho de Filho et al. (2005). Desta forma, as cargas do MEC são 
transformadas e forças nodais reativas no MEF.

No Capítulo 6, diversos exemplos são apresentados para demonstrar a eficiência e abrangência da ferramenta numérica desenvolvida. Em todos os exemplos foram utilizados os ECIs desenvolvidos neste trabalho, permitindo que resultados precisos fossem obtidos com um número de graus de liberdade relativamente reduzido.

Como a formulação aqui desenvolvida para as estacas é semelhante à de Filho et al. (2005), em todas as comparações feitas com este autor os valores obtidos resultaram muito próximos. Um exemplo desta proximidade são os gráficos 6.3 e 6.4 nos quais é difícil distiguir as curvas obtidas. Nos exemplos seguintes, nos quais são feitas comparações com outros autores, a concordância dos resutados pode ser considerada satisfatória. No caso do exemplo 6.2.3 era esperado que o resultado da formulação com ECIs seria o mais flexível, pois os demais autores apresentados restringem as bordas da malha nas direções radiais.

Nos exemplos em que foram consideradas lâminas apoiadas no maciço de solos, os resultados tanto em deslocamento como em momento fletor estão coerentes com os de outros autores. No exemplo 6.3.1 houve maior concordância com Almeida (2003b) nos deslocamentos, o que pode ser explicado pela semelhança entre as formulações. Os resultados de momento fletor também foram próximos aos de outros autores. Algo semelhante pode ser observado no exemplo 6.3.2, em que os resultados em deslocamento são próximos aos de Almeida e Paiva (2004a) e em momento próximos aos de Sadecka (2000). Por fim, no exemplo 6.3.3 é apresentado um problema envolvendo várias camadas de solo. Apesar dos resultados dos diversos autores não serem tão próximos quanto nos demais exemplos, eles podem ser considerados coerentes.

Na sequência, são apresentadas aplicações nas quais são consideradas placas com estacas. Os resultados obtidos no primeiro exemplo, no qual uma placa com uma estaca é analisada, apresentaram grande concordância com Paiva e Trondi (1999) e Butterfield e Banerjee (1971), com erros inferiores a 1 \%. Este exemplo foi complementado reduzindose o módulo de elasticidade da placa e da estaca, as quais eram inicialmente rígidas. Desta forma, foi possível analisar o comportamento da placa fletida sob diferentes carregamentos. No exemplo seguinte também houve grande concordância com Paiva e Trondi (1999), estando o resultado um pouco mais distante do obtido por Butterfield e Banerjee (1971). Também neste caso foi reduzido o módulo de elasticidade da placa e estacas rígidas, para 
então estudar a flexão da placa.

O Capítulo 6 é encerrado com dois exemplos que podem ser considerados um só dividido em duas partes. Na primeira parte, apresentada na Seção 6.4.3, um complexo conjunto formado por uma placa com nove estacas e três camadas de solo é analisado, considerando dois tipos de carregamento. Na segunda parte, apresentada na Seção 6.5. um edifício é apoiado neste sistema, compondo um problema completo de interação entre solo, estacas, radier e edifício. Apesar de não terem sido feitas comparações com outros autores, os valores obtidos nestes dois últimos exemplos podem ser considerados coerentes. Chegou-se à conclusão final de que a desconsideração da flexibilidade da fundação no cálculo de edificações pode omitir importantes excentricidades e consequente esforços na estrutura, os quais podem alterar as previsões calculadas em projeto.

\subsection{Propostas para trabalhos futuros}

Nesta Seção, são citados os principais tópicos que podem ser desenvolvidos para a continuidade deste projeto de doutoramento:

1. Associar técnicas de processamento paralelo ao programa computacional;

2. Extensão da técnica alternativa de subregiões, apresentada neste trabalho para o cálculo de deslocamentos, ao cálculo de tensões em pontos internos;

3. Consideração de não linearidade geométrica no edifício;

4. Inclusão da consideração de estacas inclinadas no solo;

5. Consideração do escorregamento das estacas em relação ao solo;

6. Desenvolvimento de uma formulação que permita dividir as estacas em vários elementos finitos;

7. Consideração de não linearidade física no solo;

8. Adaptação da técnica alternativa de subregiões para a consideração de estacas cruzando os contatos entre camadas; 


\section{Referências Bibliográficas}

Abdel-Fattah, T. T., Hodhod, H. A., e Akl, A. Y. (2000). A novel formulation of infinite elements for static analysis. Computers and Structures, 77(4):371-379. Citado em:5

Almeida, F. P. A. (2003a). Aplicação do acoplamento entre o MEC e o MEF para o estudo da interação dinâmica elastoplástica entre o solo e estruturas. Tese de Doutorado, Escola de Engenharia de São Carlos, Universidade de São Paulo, São Carlos, Brasil. Citado em:13

Almeida, V. S. (2003b). Análise da interação solo não-homogêneo/estrutura via acoplamento MEC/MEF. Tese de Doutorado, Escola de Engenharia de São Carlos da Universidade de São Paulo, São Carlos, Brasil. Citado em 24, 38, 42, 53, 159, 177, 197

Almeida, V. S. e Paiva, J. B. (2004a). Análise da interação solo não-homogêneo/fundação empregando o mec juntamente com a técnica da rigidez sucessiva. XXXI Jornadas Sud-Americanas de Ingeniería Estructural. Citado em:197

Almeida, V. S. e Paiva, J. B. (2004b). A mixed bem-fem formulation for layered soilsuperstructure interaction. Engineering analysis with boundary elements, 28:1111-1121. Citado em:12, 179

Almeida, V. S. e Paiva, J. B. (2007). Static analysis of soil/pile interaction in layered soil by bem/bem coupling. Advances in Engineering Software, 38(11-12):835-845. Citado em:12, $173,174,175$

Antunes, H. M. C. C. e Iwamoto, R. K. (2000). Comparação entre resultados observados in situ e modelos numéricos para a interação solo-estrutura. In Simpósio de Interação Estrutura-Solo em Edifícios, São Carlos. SGS/EESC-USP. Citado em:11 
Aoki, N. e Lopes, F. R. (1975). Estimating stress and settlements due to deep foundation. In Conf. Panam, volume 5, p. 377-386, Buenos Aires. Soil Mechanics and Foundation Engineering, HUELLA - Estudio Grafico. Citado em:11

Assan, A. E. (2003). Método dos elementos finitos primeiros passos. UNICAMP, Campinas, Brasil, 2 edition. Citado em 33, 35, 36, 37, 38, 47, 135, 138

Aun, G. V. (2004). A influência da largura de uma vala na determinação do empuxo passivo. Dissertação de Mestrado, Escola Politécnica, Universidade de São Paulo, São Paulo, Brasil. Citado em 13

Bae, G. J., Shin, H. S., Sicilia, C., Choi, Y. G., e Lim, J. J. (2005). Homogenization framework for three-dimensional elastoplastic finite element analysis of a grouted piperoofing reinforcement method for tunnelling. International Journal for Numerical and Analytical Methods in Geomechanics, 29:1-24. Citado em:4

Banerjee, P. K. (1976). Integral equation methods for analysis of piece-wise nonhomogeneous three-dimensional elastic solids of arbitrary shape. International Journal of Mechanical Sciences, 18(6):293-303. Citado em:7, 9

Banerjee, P. K. (1978). Analysis of axially and laterally loaded pile groups. In Developments in Soil Mechanics - I, chapter 9, p. 317-343. Applied Science Publishers, U. K.. Citado em:7

Banerjee, P. K. e Davies, T. G. (1977). Analysis of pile groups embedded in gibson soil. In Proc. 9th Int. Conf. Soil Mechs Fdn Engng., volume 1, p. 381-386, Tokyo. Citado em 9

Barbirato, J. C. C. (1999). Método dos elementos de contorno com a reciprocidade dual para a análise transiente tridimensional da mecânica do fraturamento. Tese de Doutorado, Escola de Engenharia de São Carlos da Universidade de São Paulo, São Carlos, Brasil. Citado em 38, 42, 43

Barretto, S. F. A. (1995). Análise elastodinâmica de placas através do método dos elementos de contorno com interação solo-estrutura. Tese de Doutorado, Escola de Engenharia de São Carlos, Universidade de São Paulo, São Carlos, Brasil. Citado em:13 
Barros, B. F. (2002). Métodos sem malha e método dos elementos finitos generalizados em análise não-linear de estruturas. Tese de Doutorado, Escola de Engenharia de São Carlos, Universidade de São Paulo, São Carlos. Citado em2

Barros, R. M. (1996). Elementos infinitos para o tratamento de problemas da viscoelastodinâmica estacionária em domínios elimitados pelo método dos elementos finitos. Dissertação de Mestrado, Universidade Estadual de Campinas, Campinas, Brasil. Citado em:

Barros, R. M. e Mesquita, E. (1997). Um elemento infinito para modelar a condição de radiação na interação dinâmica solo-estrutura bi-dimensional. In anais das XXVII Jornadas Sul-Americanas de Engenharia Estrutural, Sao Carlos. Citado em 5

Bathe, J. K. (1982). Finite element procedures in engineering analysis. Prentice-Hall, Englewood Cliffs. Citado em2

Batoz, J. L. (1980). A study of tree-node triangular plate bending elements. International Journal of Numerical Methods in Engineering, 15:1771-1812. Citado em2, 154

Beer, G. (2001). Programming the boundary element method. Wiley, New York. Citado $\mathrm{em}: 7$

Beer, G. e Meek, J. L. (1981). Coupled finite element-boudary element analysis of finite domain problems in geomecanics. In Hinton, E., Bettess, P., e Lewis, R. W., editors, Numerical methods for coupled problems, p. 605-629. Pineridge Press, Swansea. Citado em 9

Beer, G. e Watson, J. O. (1989). Infinite boundary elements. International Journal for Numerical Methods in Engineering, 28(6). Citado em:10

Bergan, P. G. e Felippa, C. A. (1985). A triangular membrane element with rotacional degrees of freedom. Computer Methods in Applied Mechanics and Engineering, 50:2569. Citado em 154

Bettess, P. (1977). Infinite elements. International Journal for Numerical Methods in Engineering, 11(1):53-64. Citado em5, 93, 94 
Bettess, P. (1992). Infinite elements. Penshaw Press, Sunderland, UK. Citado em 91, 97 , 103, 104,105

Bezerra, D. P. (1995). Análise de estruturas tridimensionais de edifícios altos considerando a rigidez transversal à flexão das lajes. Dissertação de Mestrado, Escola de Engenharia de São Carlos, Universidade de São Paulo, São Carlos, Brasil. Citado em2

Booker, J. R., Carter, J. P., e Small, J. C. (1989). Some recent applications of numerical methods to geotechnical analysis. Computers and Structures, 31(1):81-92. Citado em 4

Brebbia, C. A. e Dominguez, J. (1992). Boundary elements an introductory course. Computational Mechanics Publications, London. Citado em:7, 9, 15, 22, 67

Brebbia, C. A. e Georgiou, P. (1979). Combination of boundary and finite elements in elastostatics. Applied Mathematical Modelling, 3(3):212-220. Citado em 9

$\mathrm{Bu}, \mathrm{S}$. (1997). Infinite boundary elements for the dynamic analysis of machine foundations. International Journal for Numerical Methods in Engineering, 40(21):3901-3917. Citado em:10

Bu, S. H. (1996). 3d boundary element analysis of axisymmetric halfspace problems. Engineering analysis with boundary elements, 17(1):75-84. Citado em:10

Burmister, D. M. (1945a). The general theory of stresses and displacements in layered systems 1. Journal of applied physics, 16(2):89-96. Citado em 3, 131

Burmister, D. M. (1945b). The general theory of stresses and displacements in layered systems 3. Journal of applied physics, 16(5):296-302. Citado em3

Butterfield, R. e Banerjee, P. K. (1971). The problem of pile group-pile cap interaction. Géotechnique, 21(2):135-142. Citado em:7, 182, 185, 197

Chan, K. S., Karasudhi, P., e Lee, S. L. (1974). Force at a point in the interior of a layered elastic half-space. Int. J. Solids Structs., 10:1179-1199. Citado em:3, 8

Chen, L. e Poulos, H. G. (1993). Analysis of pile-soil interaction under lateral loading using infinite and finite elements. Computers and Geotechnics, 15(4):189-220. Citado em 
Cheung, Y. K., Tham, L. G., e Guo, D. J. (1988). Analysis of pile group by infinite layer method. Géotechnique, 38(3):415-431. Citado em:4

Cheung, Y. K. e Zienkiewicz, O. C. (1965). Plates and tanks on elastic foundations - an application of finite element method. Int. J. Solids Structs, 1:451-461. Citado em3

Chin, J. T. e Chow, Y. K. (1990). Numerical analysis of axially loaded vertical piles and pile groups. Computers and Geotechnics, 9:273-290. Citado em 8

Chow, Y. K. e Teh, C. I. (1991). Pile-cap-pile-group interaction in nonhomogeneous soil. Journal of Geotechnical Engineering, 117(11):1655-1668. Citado em:4

Chuhan, Z., Feng, J., e Pekau, O. A. (1995). Time-domain procedure of fe-be-ibe coupling for seismic interaction of arch dams and canyons. Earthquake Engineering and Structural Dynamics, 24(12):1651-1666. Citado em:10

Davies, T. G. e Banerjee, P. K. (1978). Displacement field due to a point load at the interface of a 2 layer elastic half-space. Géotechnique, 28(1):43-56. Citado em3

Davies, T. G. e Bu, S. (1996). Infinite boundary elements for the analysis of halfspace problems. Computers and Geotechnics, 19(2):137-151. Citado em:10

Davis, P. J. e Rabinowitz, P. (1975). Methods of numerical integration. Academic Press, New York. Citado em 98, 111

Duarte, C. A., Babusca, I., e Oden, J. T. (2000). Generalized finite element methods for three-dimensional structural mechanics problems. Computers and Structures, 77(2):215-232. Citado em 2

Filho, R. M. (1999). Análise da interação estaca-solo via combinação do método dos elementos finitos com o método dos elementos de contorno. Dissertação de Mestrado, Escola de Engenharia de São Carlos da Universidade de São Paulo, São Carlos, Brasil. Citado em56

Filho, R. M., Mendonça, A. V., e Paiva, J. B. (2005). Static boundary element analysis of piles submitted to horizontal and vertical loads. Engineering Analysis with Boundary Elements, 29:195-203. Citado em:1, 2, 12, 56, 169, 170, 171, 172, 173, 196, 197 
Fraser, R. A. e Wardle, L. J. (1976). Numerical analysis of rectangular rafts on layered foundations. Géotechnique, 26(4):613-630. Citado em5, 179, 181

Frigerio, G. P. (2004). Retroanálise de uma escavação de vala escorada a céu aberto de uma linha do metrô de são paulo. Dissertação de Mestrado, Escola de Engenharia de São Carlos, Universidade de São Paulo, São Carlos, Brasil. Citado em:13

Gao, X. W. e Davies, T. G. (1998). 3-d infinite boundary elements for half-space problems. Engineering Analysis with Boundary Elements, 21(3):207-213. Citado em:10

Gibson, R. E. (1967). Some results concerning displacements and stresses in a nonhomogeneous elastic half-space. Géotechnique, 17(1):58-67. Citado em3, 89, 195

Gibson, R. E. (1974). The analytical method in soil mechanics. Géotechnique, 24:115-140. Citado em 3

Guiggiani, M. e Gigante, A. (1990). A general algorithm for multidimensional cauchy principal value integrals in the boundary element method. Journal of Applied Mechanics, 57:906-915. Citado em $37,42,43,120,123$

Gusmão, A. D. (1990). Estudo da interação solo-estrutura e sua influência em recalques de edificações. Tese de Doutorado, Universidade Federal do Rio de Janeiro, COPPE. Citado em:11

Hetenyi, M. A. (1950). A general solution for the bending of beams on an elastic foundation of arbitrary continuity. Journal of Applied Physics, 21(1):55-58. Citado em 3

Holanda, O. G. J. (1998). Interação solo-estrutura para edifícios de concreto armado sobre fundações diretas. Dissertação de Mestrado, Escola de Engenharia de São Carlos, Universidade de São Paulo, São Carlos, Brasil. Citado em:11

Iwamoto, R. K. (2000). Alguns aspectos dos efeitos da interação solo-estrutura em edifícios de múltiplos andares com fundação profunda. Dissertação de Mestrado, Escola de Engenharia de São Carlos, Universidade de São Paulo, São Carlos, Brasil. Citado em: 13

Jordão, D. R. (2003). Estabilidade global de edifícios sobre fundações profundas, considerando a interação estrutura - solo. Dissertação de Mestrado, Escola de Engenharia de São Carlos, Universidade de São Paulo, São Carlos, Brasil. Citado em:13 
Kagawa, Y., Yamabuchi, T., e Araki, Y. (1985). The infinite boundary element and its application to the unbounded helmoltz problem. COMPEL, 1(4):29-41. Citado em:10

Kagawa, Y., Yamabuchi, T., e Kitagami, S. (1983). The infinite boundary element method and its application to a combined finite boundary element technique for unbounded field problems. COMPEL, 2(4):179-193. Citado em:10

Karakus, M., Ozsan, A., e Basarir, H. (2007). Finite element analysis for the twin metro tunnel constructed in ankara clay, turkey. Bulletin of Engineering Geology and the Environment, 66:71-79. Citado em:4

Kasper, T. e Meschke, G. (2004). A 3d finite element simulation model for tbm tunnelling in soft ground. International Journal for Numerical and Analytical Methods in Geomechanics, 28:1441-1460. Citado em 4

Kerr, A. D. (1964). Elastic and viscoelastic foundation models. Journal Applied Mechanic Transactions, ASME, 31:491-498. Citado em 3

Kerr, A. D. (1965). A study of a new foundation model. Acta Mechanic, 1:135-147. Citado em 3

Koh, K. H. e Lee, S. R. (1998). p-version static infinite element for representing $1 / r(n)$ type decay problems in unbounded media. Computers and Geotechnics, 22(1):73-89.

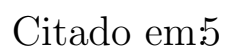

Kolar, V. e Nemec, I. (1983). Complex automatization of calculations of technical structure interaction problems. In Conf. SVTS Kosice. Citado em:179

Kumar, P. (1984). Novel infinite boundary elements. International Journal for Numerical Methods in Engineering, 20(6):1173-1173. Citado em:10

Kumar, P. (1985). Static infinite element formulation. Journal of Structural Engineering, 111(11):2355-2372. Citado em5

Kumar, P. (2000). Infinite elements for numerical analysis of underground excavations. Tunnelling and Underground Space Technology, 15(1):117-124. Citado em 5

Lee, C. Y. (1993). Pile group settlement analysis by hybrid layer approach. Journal of Geotechnical Engineering, 119(6):984-997. Citado em:3 
Lee, C. Y. e Small, J. C. (1991). Finite layer analysis of laterally loaded piles in cross-anisotropic soils. International Journal for Numerical Methods in Geomechanics, 15(11):785-808. Citado em: 4

Leite, L. G. S., Coda, H. B., e Venturini, W. S. (2001). Interação entre domínios bidimensionais e barras através do método dos elementos de contorno. In XXI CILAMCECongresso Íbero Latino de Métodos Computacionais em Engenharia, p. 1-13, Campinas. UNICAMP. Citado em-11

Liang, J. e Liew, K. M. (2001). Boundary elements for half-space problems via fundamental solutions: A three-dimensional analysis. International Journal for Numerical Methods in Engineering, 52(11):1189-1202. Citado em:10

Liu, D. S., Chiou, D. Y., e Lin, C. H. (2003). 3d iem formulation with an iem/fem coupling scheme for solving elastostatic problems. Advances in Engineering Software, 34(6):309-320. Citado em5

Liu, M. e Farris, T. N. (1993). 3-dimensional infinite boundary elements for contact problems. International Journal for Numerical Methods in Engineering, 36(19):33813398. Citado em 10

Liu, W. M. e Novak, M. (1991). Soil pile cap static interaction analysis by finite and infinite elements. Canadian Geotechnical Journal, 28(6):771-783. Citado em:5

Lopes, A. P. (2003). Análise estática e dinâmica de problemas de interação solo-estrutura utilizando os métodos dos elementos finitos e infinitos. Dissertação de Mestrado, Faculdade de Tecnologia, Universidade de Brasília, Brasília, Brasil. Citado em5

Lopes, A. P., Partridge, P. W., e Sahlit, C. L. (2003). Análise dinâmica de problemas de interação solo-estrutura utilizando elementos finitos e infinitos. In anais do XXIV CILAMCE, Ouro Preto. Citado em:5

Lopes, A. P., Sahlit, C. L., e Partridge, P. W. (2005). Análise estática de problemas de interação solo-estrutura utilizando elementos finitos e infinitos. In anais do XXVI CILAMCE, Guaraparí. UFES. Citado em5 
Lorentz, R. G. (1985). Alguns aspectos na interação estrutura-solo-estrutura. Dissertação de Mestrado, Pontificia Universidade Catolica do Rio de Janeiro, Rio de Janeiro, Brasil. Citado em:13

Love, A. E. H. (1944). A treatise on the mathematical theory of elasticity. Dover, New York, 4 edition. Citado em 9 , 21

Maier, G. e Novati, G. (1987). Boundary element elastic analysis by a successive stiffness method. International Journal for Numerical and Analytical Methods in Geomechanics, 11(5):435-447. Citado em 8,12

Marques, J. M. M. C. e Owen, D. R. J. (1984). Infinite elements in quasi-static materially nonlinear problems. Computers and Structures, 18(4):739-751. Citado em5, 103

Mattes, N. S. e Poulos, H. G. (1969). Settlement of single compressible pile. Journal of the Soil Mechanics and Foundations Division, 95(1):189-207. Citado em6

Medeiros, L. H. A. (1994). Elementos infinitos mapeados e elementos virtuais no cálculo de campos eletromagnéticos em problemas com fronteiras abertas pelo método de elementos finitos. Dissertação de Mestrado, Universidade Federal de Santa Catarina, Brasil. Citado em

Medeiros, L. H. A. e Raizer, A. (1994). Análise de problemas com fronteiras abertas. uso de elementos infinitos mapeados acoplado à técnica de elementos finitos. In anais do $10^{\circ}$ Congresso Brasileiro de Automática, Rio de Janeiro. Citado em:5

Meek, J. L. (1988). Becoup - a program for coupled boundary and finite element analysis in 3d elastostatics. In Brebbia, C. A., editor, Boundary elements X: stress analysis, volume 3, p. 639-655. CML Publ., Southampton. Citado em9

Mello, G. J. T. (1984). Interação inercial solo-estrutura para subleitos estratificados. Dissertação de Mestrado, Pontificia Universidade Catolica do Rio de Janeiro, Rio de Janeiro, Brasil. Citado em:13

Mendonça, A. V. (1997). Análise da interação placa-estaca-solo via combinação do método dos elementos finitos com o método dos elementos de contorno. Dissertação de Mestrado, Escola de Engenharia de São Carlos, Universidade de São Paulo, São Carlos, Brasil. Citado em 13 
Mendonça, A. V. (2002). Estudo de estruturas compostas por lâminas planas: uma abordagem pelo método dos elementos de contorno. Tese de Doutorado, Escola de Engenharia de São Carlos, Universidade de São Paulo, São Carlos. Citado em2

Mendonça, A. V. e Paiva, J. B. (2000). A boundary element method for the static analysis of raft foundations on piles. Engineering analysis with boundary elements, 24(3):237247. Citado em:12

Mendonça, A. V. e Paiva, J. B. (2003). A elastostatic fem/bem analysis of vertically loaded raft and piled raft foundation. Engineering analysis with boundary elements, 27:919-933. Citado em 12

Mesquita, A. D. (1998). Uma formulação do método dos elementos finitos aplicada á analise elastoplástica de cascas. Dissertação de Mestrado, Escola de Engenharia de São Carlos, Universidade de São Paulo, São Carlos, Brasil. Citado em2

Mesquita, A. D. e Coda, H. B. (2000). Análise viscoelástica da interação solo-estrutura, representação bidimensional. In Simpósio de Interação Estrutura-Solo em Edifícios, São Carlos. Programas de Pós-Graduação em Geotecnia e Engenharia de Estruturas da USP/SC, SET/EESC-USP. Citado em:11

Mesquita, E. e Barros, R. M. (1995). Elementos infinitos unidimensionais e o modelamento da condição de radiação em sistemas elastodinâmicos. In anais do COBEM/CIDIM 95, Belo Horizonte. Citado em

Messafer, T. e Coates, L. E. (1989). Advances in boundary methods. In Brebbia, C. e Connor, editors, An application of FEM/BEM coupling to foundation analysis, p. 211221. Computational Mechanics Publications, Southampton and Boston. Citado em 177

Mindlin, R. D. (1936). Force at a point in the interior of a semi-infinite solid. J. Physics, 7:195-202. Citado em.6. 9

Moser, W., Duenser, C., e Beer, G. (2004). Mapped infinite elements for three-dimensional multi-region boundary element analysis. International Journal for Numerical Methods in Engineering, 61(3):317-328. Citado em:11, 104, 116, 129

Moura, A. R. L. U. (1995). Interação solo-estrutura em edifícios. Dissertação de Mestrado, Universidade Federal de Pernambuco, Recife, Brasil. Citado em:11 
Moura, A. R. L. U. (1999). Análise tridimensional de interação solo - estrutura em edifícios. Solos e Rochas, 22(2):87-100. Citado em:13

Mylonakis, G. e Gazetas, G. (1998). Settlement and additional internal forces of grouped piles in layered soil. Géotechnique, 48(1):55-72. Citado em3

Nakaguma, R. K. (1979). Three-dimensional elastostatics using the boundary element method. Tese de Doutorado, University of Southampton, New York, USA. Citado em9

Neto, V. C. (2005). Interação de paredes em alvenaria estrutural cerâmica sob ações verticais. Tese de Doutorado, Escola de Engenharia de São Carlos, Universidade de São Paulo, São Carlos, Brasil. Citado em:13

Oñate, E. I. N. (1995). Cálculo de estructuras por el método de los elementos finitos. Relatório técnico, Centro Internacional de Métodos Numéricos en Ingeniería, Barcelona, Espanha. Citado em2, 25, 38

Oshima, S. T. (2004). Uma combinação mec/mef para análise da interação de estacas inclinadas e o solo. Dissertação de Mestrado, Escola de Engenharia de São Carlos, Universidade de São Paulo, São Carlos, Brasil. Citado em:13

Ottaviani, M. (1975). Three-dimensional finite element analysis of vertically loaded pile groups. Géotechnique, 25(2):159-174. Citado em:4, 173, 174, 175

Paccola, R. R. (2004). Análise não linear física de placas e cascas anisotrópicas laminadas acopladas ou não com meio contínuo tridimensional viscoelástico através da combinação entre o MEC e o MEF. Tese de Doutorado, Escola de Engenharia de São Carlos, Universidade de São Paulo, São Carlos, Brasil. Citado em:13

Paiva, J. B. e Aliabadi, M. H. (2000). Boundary element analysis of zoned plates in bending. Computational Mechanics, 25(6):560-566. Citado em 8,67

Paiva, J. B. e Aliabadi, M. H. (2004). Bending moments at interfaces of thin zoned plates with discrete thickness by the boundary element method. Engineering analysis with boundary elements, 28:747-751. Citado em 8, 67

Paiva, J. B. e Butterfield, R. (1997). Boudary element analysis of plate-soil interaction. Computers and Structures, 64(1-4):319-328. Citado em:177 
Paiva, J. B. e Trondi, R. R. (1999). Boundary element analysis of capped and uncapped pile groups. Advances in Engineering Software, 30(9-11):715-724. Citado em182, 185. 197

Pan, E. (1997). Static green's functions in multilayered half spaces. Applied Math. Modelling, 21(8):509-521. Citado em 8

Peleteiro, S. C. (1996). Utilização da formulação livre para desenvolvimento de um elemento de membrana com liberdades rotacionais. Dissertação de Mestrado, Escola de Engenharia de São Carlos, Universidade de São Paulo, São Carlos, Brasil. Citado em2

Pereira, U. A. (2004). Deformabilidade de estruturas em solos reforçados : influência da proximidade de construções vizinha. Dissertação de Mestrado, Universidade de Brasília, Brasília, Brasil. Citado em: 13

Poulos, H. G. (1967). Stresses and displacements in an elastic layer underlain by rough rigid base. Géotechnique, 17(4):378-410. Citado em 3, 6, 11

Poulos, H. G. (1968). Analysis of the settlement of pile groups. Géotechnique, 18(3):449471. Citado em.6, 11

Poulos, H. G. (1971a). Behaviour of laterally loaded piles: 1 - single piles. Journal of the Soil Mechanics and Foundations Division, 97(5):711-731. Citado em:7

Poulos, H. G. (1971b). Behaviour of laterally loaded piles: 2 - pile groups. Journal of the Soil Mechanics and Foundations Division, 97(5):733-751. Citado em:7

Poulos, H. G. e Davies, H. G. (1968). The settlement behaviour of single axially loaded incompressible piles and piers. Géotechnique, 18:351-371. Citado em[6]

Quezado, L. (1993). Proposição de um modelo físico para estudo da interação soloestrutura em fundações profundas. Tese de Doutorado, Universidade de Brasília, Brasília, Brasil. Citado em:13

Randolph, M. F. e Wroth, C. P. (1979). An analysis of the vertical deformation of pile groups. Géotechnique, 29(4):423-439. Citado em3 
Rangel, A. B. C. (1997). Análise numérica de um modelo físico para fundações em solocimento. Tese de Doutorado, Universidade de Brasília, Brasília, Brasil. Citado em: 13

Reis, J. H. C. (2000). Interação solo-estrutura de grupos de edifícios com fundações superficiais em argila mole. Dissertação de Mestrado, Escola de Engenharia de São Carlos, Universidade de São Paulo, São Carlos, Brasil. Citado em:11

Ribeiro, D. B. (2005). Análise da interação solo-estrutura via acoplamento mec/mef. Dissertação de Mestrado, Escola de Engenharia de São Carlos, Universidade de São Paulo, São Carlos, Brasil. Citado em:1, 12, 155

Ribeiro, D. B., Almeida, V. S., e Paiva, J. B. (2005a). Análise via mec 3d da interação de blocos com um solo finito. In anais do XXVI CILAMCE, Guaraparí. UFES. Citado em 12

Ribeiro, D. B., Almeida, V. S., e Paiva, J. B. (2005b). Uma formulação alternativa para analisar a interação solo não-homogêneo/ fundação/ superestrutura via acoplamento mec-mef. Revista Sul-Americana de Engenharia Estrutural, 2(2):27-46. Citado em:12

Ribeiro, D. B. e Paiva, J. B. (2008). Uma nova formulação de elementos de contorno infinitos para análise de sólidos 3d. In anais do XXIX CILAMCE, Maceió. UFAL. Citado em:1

Ribeiro, D. B. e Paiva, J. B. (2009). An alternative multi-region bem technique for three-dimensional elastic problems. Engineering Analysis with Boundary Elements, 33(4):499-507. Citado em:1, 8, 195

Rios, B. M. C. (1991). Análise tridimensional e envoltória de esforços em edifícios altos sujeitos a ações verticais e laterais. Dissertação de Mestrado, Escola de Engenharia de São Carlos, Universidade de São Paulo, São Carlos, Brasil. Citado em2

Sadecka, L. (2000). A finite/infinite element analysis of thick plate on a layered foundation. Computers and Structures, 76(5):603-610. Citado em:5, 179, 197

Silva, M. T. (1992). Interação solo-estrutura de fundação em estacas de solo-cimento e concreto. Dissertação de Mestrado, Universidade de Brasília, Brasília, Brasil. Citado em 13 
Silva, M. T. (1994). Interação solo-estrutura de fundação em estacas de solo-cimento e concreto. Tese de Doutorado, Universidade de Brasília, Brasília, Brasil. Citado em:13

Singh, R. B., Sharma, K. G., e Varadarajan, A. (1988). Elasto-plastic analysis of tunnel excavation in layred rock medium by coupled febem. Numerical Methods in Geomechanics, p. 941-950. Citado em9

Small, J. C. e Booker, J. R. (1984). Finite layer analysis of layered elastic materials using a flexibility approach. part 1 - strip loadings. International Journal for Numerical Methods in Engineering, 20(6):1025-1037. Citado em:4

Soares, J. M. (2004). Estudo númerico-experimental da interação solo-estrutura em dois edifícios do Distrito Federal. Tese de Doutorado, Universidade de Brasília, Brasília, Brasil. Citado em 13

Southcott, P. H. e Small, J. C. (1996). Finite layer analysis of vertically loaded piles and pile groups. Computer and Geotechnics, 18(1):47-63. Citado em:4

Swoboda, G., Mertz, W., e Beer, G. (1987). Rheological analysis of tunnel excavation by means of coupled finite element (fem)-boundary element (bem) analysis. International Journal for Numerical and Analytical Methods in Geomechanics, 11(2):115-129. Citado em 9

Ta, L. D. e Small, J. C. (1998). Analysis and performance of piled raft foundations on layered soils-case studies. Soil and Foundations, 38(4):145-150. Citado em:4

Ulusay, R., Aksoy, H., e Ider, M. H. (1993). Geotechnical approaches for the design of a railway tunnel section in andesite. Engineering Geology, 34:81-93. Citado em 4

Ungless, R. L. (1973). An infinite finite element. Dissertação de Mestrado, University of British Columbia. Citado em 92

Veiga, J. (2000). Análise de problemas tridimensionais solo-estrutura pelo método dos elementos finitos no domínio de fourier. Dissertação de Mestrado, Pontificia Universidade Catolica do Rio de Janeiro, Rio de Janeiro, Brasil. Citado em:13

Venturini, W. S. (1988). Um estudo sobre o método dos elementos de contorno e suas aplicações em problemas de engenharia. Tese (livre docência) - Escola de Engenharia de São Carlos, Universidade de São Paulo. Citado em56 
Venturini, W. S. (1992). Alternative formulations of the boundary element method for potential and elastic problems. Engineering Analysis with Boundary Elements, 9:203207. Citado em 8,67

Wang, C. M., Iang, Y. X., e Wang, Q. (2001). Axisymmetric buckling of reddy circular plates on pasternak foundation. Journal Engineering Mechanic, 127(3):254-259. Citado em

Wardle, L. J. e Fraser, R. A. (1974). Finite element analysis of a plate on a layered cross-anisotropic foundation. In Proceedings of the First International Conference of Finite Element Methods in Engineering, p. 565-578, University of New South Wales, Australia. Citado em 181

Witt, M. (1984). Solutions of plates on a heterogeneous elastic foundation. Computers and Structures, 18(1):41-45. Citado em 3

Xiaoping, L. e Wei-liang, W. (2005). A new subregion boundary element technique based on the domain decomposition method. Engineering Analysis with Boundary Elements, 29:944-952. Citado em:7

Xiaoping, L. e Wei-liang, W. (2006). A subregion drbem formulation for the dynamic analysis of two-dimensional cracks. Mathematical and Computer Modeling, 43:76-88. Citado em: 8

Y. H. Wang, L. G. Tham, Y. T. e Yue, Z. Q. (2003). Plate on layered foundation analyzed by a semi-analytical and semi-numerical method. Computers and Geotechnics, 30:409418. Citado em:181

Yang, S. C. e Yun, C. B. (1992). Axisimmetrical infinite elements for soil-structure interaction analysis. Engineering Structures, 14(6):361-370. Citado em:5

Yerli, H. R., Kacin, S., e Kocak, S. (2003). A parallel finite-infinite element model for two-dimensional soil-structure interaction problems. Soil Dynamics and Earthquake Engineering, 23(4):249-253. Citado em:5

Yerli, H. R., Temel, B., e Kiral, E. (1998). Transient infinite elements for 2d soilstructure interaction analysis. Journal of Geotechnical and Geoenviromental Engeneering, 124(10):976-988. Citado em5 
Yin, L. Z. e Yang, W. (2000). Topology optimization for tunnel support in layered geological structures. International Journal for Numerical Methods in Engineering, 47:19831996. Citado em 4

Yun, C. B., Choi, J. S., e Kim, J. M. (1999). Identification of the hualien soil-structure interaction system. Soil Dynamics and Earthquake Engineering, 18(6):395-408. Citado em[5

Zhang, C. H., Chen, X. F., e Wang, G. L. (1999). A coupling model of fe-be-ie-ibe for non-linear layered soil-structure interactions. Earthquake Engineering and Structural Dynamic, 28(4):421-441. Citado em:10

Zhang, C. H., Pekau, O. A., e Jin, F. (1992). Application of fe-be-ibe coupling to dynamic interaction between alluvial soil and rock canyons. Earthquake Engineering and Structural Dynamics, 21(5):367-385. Citado em-10

Zhang, C. H., Song, C. M., e Pekau, O. A. (1991). Infinite boundary elements for dynamic problems of 3-d half-space. International Journal for Numerical Methods in Engineering, 31(3):447-462. Citado em:10

Zhang, C. H., Song, C. M., Wang, G. L., e Jin, F. (1989). 3-d infinite boundary elements and simulation of monolithic dam foundations. Communications in Applied Numerical Methods, 5(6):389-400. Citado em:10

Zhu, W. S., Li, S. C., Li, S. C., Chen, W. Z., e Lee, C. F. (2003). Systematic numerical simulation of rock tunnel stability considering different rock conditions and construction effects. Tunnelling and Underground Space Technology, 18:531-536. Citado em 4 